\title{
Stem-, Spraak- en Taalpathologie
}

20th International Science of Aphasia Conference: Abstracts

\begin{tabular}{lc} 
Program & $\mathrm{i}$ \\
\hline Oral Presentations I & 1 \\
\hline Poster Session I & 23 \\
\hline Oral Presentations II & 65 \\
\hline Poster Session II & 80 \\
\hline Oral Presentations III & 120 \\
\hline Poster Session III & 148 \\
\hline & 190 \\
\hline
\end{tabular}




\title{
Science of Aphasia XX Rome, September 23-26, 2019
}

\section{The temporal lobe revisited: Neural and Functional updates}

\section{PROGRAM}

\section{Monday 23 September}

\author{
$9.00-12.00 \quad$ Invited talks: Anatomical and functional overview
}

Marco Catani (NatBrainLab, King's College, London, UK)

David Poeppel (Max-Planck-Institut fur empirische Aesthetik, Frankfurt, Germany)
$12.00-13.00$
Lunch
$13.00-15.00$
Oral presentations 1

1. Joanna Sierpowska, Katherine L. Bryant, Manon Römkens, Margot Mangnus, Nikki Janssen, Roy Kessels, Ardi Roelofs, Rogier Mars \& Vitoria Piai - The functional neuroanatomy of the left temporal lobe white matter -an interdisciplinary approach based on intraoperative and comparative studies

2. Jun Soo Noh, Sekwang Lee, Yoonhye Na, Minjae Cho, Yu Mi Hwang, Woo-Suk Tae \& Sung-Bom Pyun - The Role of Arcuate Fasciculus on Severity of Language Impairments in Subcortical Stroke Patients

3. Ileana Camerino, Joanna Sierpowska, Nathalie Meyer, Anil Tuladhar, Roy Kessels, Frank-Erik de Leeuw \& Vitória Piai - White-matter bottleneck in small vessel disease: A lesion-symptom mapping study of executive-language functions

4. Elissa Asp, Antoine Tremblay, Graham Flick \& Aaron Newman - CForests in the trees: Using conditional inference random forests on MEG data to explore 'what fires together' in a picture naming task

5. Wei Ping Sze, Jane Warren, Solène Hameau \& Wendy Best - What are the active ingredients in anomia therapy? A random forest analysis of anomia research from 20092018

6. Edith Durand, Pierre Berroir \& Ana Inés Ansaldo - Neural substrates associated with the recovery of verb anomia: the role of sensorimotor strategies in the improvement of naming abilities in aphasia

7. Sonia Montemurro, Gonia Jarema \& Sara Mondini - Lexical Frequency, Lexical Semantics in interaction with Cognitive Reserve in healthy older adults 
$15.00-15.30 \quad$ Coffee

$15.30-16.15 \quad$ Flash presentations poster session 1

16:15-17.00 Poster session 1

1. M. De Martino, A. Mancuso, A. G. Russo, A. Elia, F. Di Salle, R. Saponiero, S. Vietri, F. Esposito \& A. Laudanna - Inflecting regular and irregular verbs: neuroimaging and behavioural data from the three Italian conjugations

2. Johémie Boucher, Karine Marcotte, Amélie Brisebois, Marianne Désilets-Barnabé, Alberto Osa García, Elizabeth Rochon, Carol Leonard, Alex Desautels, Simona Brambati - Confrontation-naming and connected speech production in early post-stroke aphasia

3. Martina Garzon, Federica Biddau, Giorgio Arcara, Francesca Meneghello, Daniela D'Imperio, Giulia Bencini - Investigating the potential of structural priming as a form of constraint-induced language therapy in post-stroke aphasia

4. Nour Ezzeddine \& Barbara Köpke - Adaptation of the Bilingual Aphasia Test to Lebanese Arabic

5. Hüsnünur Aslantürk, Nurten Tiryaki \& Bülent Toğram - The Experiences of Aphasia Caregivers In Turkey

6. Bülent Toğram - Public Awareness of Aphasia in Turkey

7. Svetlana Malyutina, Yulia Akinina \& Valeriya Zelenkova - The subject-object-verb word order as a self-cueing strategy in aphasia: An exploratory study

8. Giulia Krethlow, Grégoire Python \& Marina Laganaro - Differences in semantic priming during lifespan and in aphasia

9. Brisotto, C., Biddau, F. \& Nordio, S. - Speech and language therapy for acquired dysgraphia in neurological patients: a systematic review

10. Sabrina Beber, Rita Capasso \& Gabriele Miceli - Neurofunctional correlates of auditory and visual sentence comprehension: Evidence from aphasia

11. Lucy Dipper, Madeline Cruice, Jane Marshall, Nicola Botting, Mary Boyle, Deborah Hersh \& Madeleine Pritchard - Characterising the Nature of Discourse Treatment in Aphasia Rehabilitation Research

12. Ingrid Aichert, Katharina Lehner, Simone Falk, Mona Späth, Mona Franke \& Wolfram Ziegler - In Time with the Beat: Entrainment in Patients with Phonological Impairment, Apraxia of speech and Parkinson's disease

13. Svetlana Kuptsova, Anastasia Ulicheva, Olga Dragoy \& Maria Ivanova - Impairment of switching attention in patients with fluent aphasia and temporal lobe damage

14. Elissa-Marie Cocquyt, Patrick Santens, Pieter van Mierlo, Wouter Duyck, Arnaud Szmalec \& Miet De Letter - Age-related differences in auditory semantic priming: the development of normative electrophysiological data in the Dutch population 


\section{Tuesday 24 September}

$9.00-12.00 \quad$ Invited talks: Auditory and music perception

Isabelle Peretz (University of Montréal, Canada)

Gabriele Miceli (CIMeC, University of Trento, and Centro Interdisciplinare Linceo, Rome)

$12.00-13.00 \quad$ Lunch

$13.00-14.15 \quad$ Oral presentations 2

1. Olga Dragoy, Ekaterina Stupina, Andrey Zyryanov, Marina Chernova, Elizaveta Gordeyeva, Natalya Gronskaya, Galina Gunenko, Sergey Chernov, Dmitry Kopachev, Igor Medyanik, Nikita Pedyash, Igor Pronin, Andrey Sitnikov, Konstantin Yashin, Andrey Zuev - 'A moderate global aphasia': the pattern of language deficits in acute post-surgical tumor patients

2. Karine Marcotte, Alberto Osa, Johémie Boucher, Bérengère Houzé, Christophe Bedetti, Amélie Brisebois, Alex Desautels \& Simona Maria Brambati - Right-hemisphere density reduction in acute post-stroke aphasia

3. Olga Buivolova, Oxana Vinter, Roelien Bastiaanse \& Olga Dragoy - Validation of the Aphasia Rapid Test in the Russian-speaking post-stroke population

4. Yulia Akinina, Olga Buivolova, Olga Soloukhina \& Roelien Bastiaanse - Psychometric Properties of the Token Test App

5. C. Jacquemot, C. Schramm, L. Lemoine, K. Youssof \& AC. Bachoud-Lévi - Coupling language and executive functions for premanifest and early Huntington's Disease follow up

14:15-14:45 Coffee

14:45-15:30 $\quad$ Flash presentations poster session 2

15:30 -16-30 Poster session 2

1. Svetlana Averina, Olga Dragoy \& Roelien Bastiaanse - The role of the white matter pathways in spontaneous speech in aphasia

2. Brianne Chiappetta, Matthew Walenski, Elena Barbieri, Aniruddh Patel \& Cynthia Thompson - Musical and linguistic syntactic processing in agrammatic aphasia: An ERP study

3. Cristina Rosazza, Maria Gazzotti, Paolo Urso, Valentina Impagnatiello, Cinzia Crivellaro \& Valeria Isella - Brain metabolic correlates of errors on picture naming in Alzheimer's Disease

4. Ikram Methqal \& Yves Joanette - When Trade-Offs in NeuroCognitive Resources Determine Word Production Efficiency in Aging

5. Liyana Low, Susan Rickard Liow, Melvin Yap, Tng Siok Keng \& Rebecca Heywood Written Language in Mandarin-dominant Older Adults with Hearing Loss

6. Roelant Ossewaarde, Roel Jonkers \& Roelien Bastiaanse - Determining the ideal length of spontaneous speech fragments for predictive analysis

7. Xabi Ansorena, Mireia Hernández, Manuel Carreiras, José Ignacio Quemada \& Simona Mancini - Short Term Memory and sentence processing in deep dysphasia 
8. Soultana Georgiadou, Stavroula Stavrakaki \& Vasileios Kimiskidis - Language abilities in Aicardi Syndrome: A case study

9. Cyrielle Demierre, Grégoire Python, Bertrand Glize, Marina Laganaro - Which word planning processes require attention? Evidence from dual-task interference in aphasia

10. Marion Bourqui, Michaela Pernon, Cécile Fougeron, \& Marina Laganaro - Contribution of acoustic analysis in the differential diagnosis of apraxia of speech

11. Mile Vuković \& Irena Vuković - The investigation of paraphasias in speakers with fluent and non-fluent aphasia

12. Mateusz Choiński, Elżbieta Szeląg, Anna Bombińska \& Aneta Szymaszek - Positive effects of a treatment based on temporal information processing on language and nonlanguage cognitive functions in individuals with aphasia: a pilot study

13. Faith Chiu \& Typhanie Prince - The production of French consonant sequences in typically developing children and in people with aphasia

14. Antje Lorenz, Danièle Pino, Jörg D. Jescheniak, Frank Regenbrecht, \& Hellmuth Obrig - Grammatical-gender effects in noun-noun compound production: Evidence from aphasia

15. Ekaterina Delikishkina, Angelika Lingnau \& Gabriele Miceli - Investigating the Neural Correlates of Argument Structure Processing

$18.00-20.00 \quad$ Tour

$20.30-23.00 \quad$ Dinner (Palazzo Corsini)

\section{Wednesday 25 September}

$9.00-12.00 \quad$ Invited talks: Semantics

Alex Martin (Section on Cognitive Neuropsychology, Laboratory of Brain and Cognition, National Institutes of Mental Health, Bethesda, MD, USA)

Costanza Papagno (CIMeC, University of Trento, and University of Milan La Bicocca, Italy)

$12.00-13.00 \quad$ Lunch

$13.00-15.00 \quad$ Oral presentations 3

1. Valantis Fyndanis, Lambros Messinis, Grigorios Nasios, Efthimios Dardiotis, Maria Martzoukou, Maria Pitopoulou, Katerina Ntoskou, Sonia Malefaki - Impaired verb-related morphosyntactic production in Multiple Sclerosis: Evidence from Greek

2. Cristina Romani, Raffaele Nappo, Ivana Bureca, \& Gaspare Galati - Semantic hyperinterference and hyper-faciliation in aphasia: Evidence for activation-based models

3. Jessica Obermeyer \& Nadine Martin - The influence of verbal short-term memory capacity on microlinguistic measures of word and utterance level content in discourse

4. Katharina Hogrefe, Wolfram Ziegler, Ralf Glindemann, Madleen Klonowski, Edith Wagner-Sonntag, Gudrun Klingenberg, Janine Diehl-Schmid, Carola Roßmeier , Adrian Danek, Johannes Levin, Catharina Prix, Sandra Loosli, Elisabeth Wlasich, \& Georg Goldenberg - Application of the Nonverbal Semantics Test (NVST) to persons with aphasia after stroke and persons with dementia

5. Kati Renvall \& Lyndsey Nickels - Treatment of adjectives in aphasia: Two case-studies

6. Lyndsey Nickels, Sharon Savage, Leonie Lampe, Olivier Piguet \& John Hodges - In- 
vestigating over-generalisation following word-retrieval treatment in Semantic Dementia

7. Ashleigh Beales, Anne Whitworth, Jade Cartwright, Peter Panegyres \& Robert Kane - Strategy, cognition, and communication partners: Maximizing treatment impact in progressive aphasia and Alzheimer's disease

8. Jasmina Vuksanović, Tanja Milovanović, Ljubica Konstantinović \& Saša R. Filipović - Effect of Type of Language Therapy on Language Improvement in Patients with PostStroke Aphasia

$15.00-15.30 \quad$ Coffee

$15.30-16.15 \quad$ Flash presentations poster session 3

16:15-17.00 Poster session 3

1. Özlem Oğuz - Communicating and fixing communication breakdowns with people with aphasia: Speech-language therapists' (SLTs) and caregivers' perspectives

2. Šešok, S., Bolle, N. \& Kobal, J. - Verbal and nonverbal fluency in presymptomatic carriers of the Huntington's disease gene

3. Typhanie Prince - Exploring Phonological Deficits in French speakers with Acute stroke Aphasia: A Preliminary Study

4. Jill Kries, Marlies Gillis, Jonas Vanthornhout, Tom Francart \& Maaike Vandermosten Neural tracking of semantics in natural speech

5. Anastasios Georgiou \& Maria Kambanaros - Neuronavigated $1 \mathrm{~Hz}$ repetitive Transcranial Magnetic Stimulation (rTMS) in Chronic post-Stroke Aphasia

6. Arushi Garg, Vitória Piai, Atsuko Takashima, James M. McQueen \& Ardi Roelofs Linking production and comprehension -Investigating the lexical interface

7. Dilek Eroğlu Uzun, Serkan Şener \& Barış Metin - The Effect of tDCS on Syntactic Processing in Aphasia

8. Dörte de Kok, Sarah Hanekamp \& Roelien Bastiaanse - Linguistic parameters in an app-based assessment of German verbs and nouns in aphasia

9. Maaike Vandermosten, Klara Schevenels, Inge Zink \& Bert De Smedt - Understanding language recovery in stroke patients by including neuroanatomical and behavioural measures of learning potential

10. Elena Salillas, Domenico D'Avella, Giorgio Arcara, Francesco Piccione, Sara Zago, Silvia di Tomasso \& Carlo Semenza - Assessing verb selection using MEG. A precise methodology for language presurgical mapping

11. Georgia Roumpea, Maja Blesić, Dejan Georgiev \& Christina Manouilidou - Investigating regular and irregular morphology in Parkinson's and Alzheimer's disease: evidence from Slovenian

12. Ida Luotonen, Kati Renvall \& Pirjo Korpilahti - Semantic memory tasks for neurogenic disorders: Data on healthy elderly adults, Alzheimer's disease and stroke aphasia

13. Nomiki Karpathiou \& Maria Kambanaros - Frontotemporal dementia: a comparative case study of Greek-speaking individuals with the non-fluent and semantic variants of primary progressive aphasia

14. Nikki Janssen, Margot Mangnus, Ardi Roelofs, Joanna Sierpowska, Roy Kessels, Vitória Piai - The role of the uncinate fasciculus and inferior longitudinal fasciculus in healthy and disordered language production 


\section{Thursday 26 September}

$9.00-12.00$

Invited talks: Language in the temporal lobe: Evolution, loss \& recovery

Laurent Cohen (ICM, Pitié Salpêtrière hospital and University of Paris, France)

Cynthia K Thompson (Department of Communication Sciences and Disorders, Northwestern University, Chicago, Il, USA)

$12.00-13.00 \quad$ Lunch

Flash presentations poster session 4

$13.45-14: 30 \quad$ Poster session 4

1. Saša Filipović, Jasmina Vuksanović, Tanja Milovanović \& Ljubica Konstantinović Effect of Type of Language Therapy on Fluency in Patients with Post-Stroke Aphasia

2. Tanja Milovanović, Jasmina Vuksanović, Ljubica Konstantinović \& Saša R. Filipović - Effects of language therapies on receptive language recovery in post-stroke aphasia patients

3. Mizoon Ali, Marian Brady et al. - RELEASE-ing the potential of a large, international, systematic review-based Individual Participant Data (IPD) aphasia after stroke database for meta- and network meta-analysis

4. Pauliina Sorvisto, Paul Mullins \& Marie-Josèphe Tainturier - Effects of orthographic depth on functional connectivity within reading pathways in proficient bilinguals

5. Vânia de Aguiar, Adrià Rofes, Bronte Ficek, Kimberly Webster, Haley Wendt \& Kyrana Tsapkini - Treating lexical retrieval using letter fluency in primary progressive aphasia - a single case study

6. Lorenzo Vercesi, Prerana Sabnis, Chiara Finocchiaro, Luigi Cattaneo, Elena Tonolli \& Gabriele Miceli - Who does what to whom: the role of the l-IPS in the comprehension of reversible and irreversible sentences

7. Rosell-Clari, V., Hernández-Sacristán, C., \& Lorenzo-Cordero, A. - Comparison of metalinguistic profiles of early-stage Alzheimer's patients and healthy older adults

8. Bruns, C., Zimmerer, V., Bruce, C., Varley, R. \& Beeke, S. - Using multi-word utterances more flexibly in non-fluent aphasia: Findings from a case series investigation

9. Maja Blesić, Dejan Georgiev \& Christina Manouilidou - Prosody perception by Slovene speaking individuals diagnosed with Parkinson's Disease

10. Julie Schlesinger, Jessica Obermeyer \& Nadine Martin - Repeated item exposure effects in a verbal short-term memory treatment

11. Matthias Sandmann, Sabine Weiss \& Horst Müller - Do you prefer playing "with fire" or "with the flame"? Idiom comprehension in individuals with mild aphasic symptoms

12. Shinri Ohta, Yohei Oseki \& Alec Marantz - Morpheme processing in the ventral temporal lobe: An MEG study of Japanese verbs

13. Ann-Katrin Ohlerth, Roelien Bastiaanse, Chiara Negwer, Nico Sollmann, Severin Schramm, Axel Schröder \& Sandro M. Krieg - Cortical and subcortical involvement during Object an Action Naming in healthy participants under nTMS

14. Carolina Méndez-Orellana, Caitlin Holme, Karina Sandoval-León, Bárbara Cortés-Rivera, Paula Méndez-Orellana, Silvia Martínez-Ferreiro - Spontaneous Speech Analysis in Spanish-Speaking Adults: Normative data in healthy adults, elderly adults and patients 
with brain lesions 
Oral Presentations I 


\title{
The functional neuroanatomy of the left temporal lobe white matter -an interdisciplinary approach based on intraoperative and comparative studies
}

\author{
Joanna Sierpowska ${ }^{1,2}$, Katherine L. Bryant ${ }^{1}$, Manon Römkens ${ }^{1}$, Margot Mangnus ${ }^{1}$, Nikki \\ Janssen $^{1,2}$, Roy P. C. Kessels ${ }^{1,2}$, Ardi Roelofs ${ }^{1}$, Rogier B. Mars ${ }^{1,3}$, Vitoria Piai ${ }^{1,2}$ \\ ${ }^{1}$ Donders Institute for Brain, Cognition and Behaviour, Radboud University, Nijmegen, The \\ Netherlands \\ ${ }^{2}$ Department of Medical Psychology, Radboud University Medical Center, Nijmegen, The \\ Netherlands \\ ${ }^{3}$ Wellcome Centre for Integrative Neuroimaging, Centre for Functional MRI of the Brain \\ (FMRIB), Nuffield Department of Clinical Neurosciences, John Radcliffe Hospital, University \\ of Oxford, Oxford, United Kingdom
}

\section{Introduction}

The left temporal lobe is claimed to be involved in language comprehension in both the classical (Wernicke, 1874) and contemporary dual-pathway models for language processing (Hickok \& Poeppel, 2007, Roelofs, 2014), but the role of neuroanatomical subcomponents of this lobe in distinct facets of lexical-semantic processing has still not been fully uncovered. This knowledge becomes critical in the context of intraoperative monitoring of language function during awake brain surgeries. Brain tumors tend to develop within the deep white matter of the left temporal lobe, compromising the correct transfer of information across these areas. In previous clinical work, we have observed that intraoperative electrical stimulation within the left temporal lobe provokes an overflow of semantic paraphasias and errors in semantic matching tasks (Sierpowska et al., 2019). This work culminated in establishing a protocol for intraoperative semantic processing monitoring, but did not convey sufficiently precise neuroanatomical insights. Therefore, voxel-lesion symptom mapping and track-wise analyses were performed. Results suggested the involvement of two critical structures in semantic processing: the posterior middle temporal gyrus (pMTG) and the inferior fronto-occipital fasciculus (IFOF). One strategy for finding supporting evidence for the functional role of these structures is the use of comparative studies. Comparative neuroscience involves the examination of brain organization of several species in evolutionary context in order to determine the relationship between brain structure and behavior. Sarubbo et al. (2019) used this approach to demonstrate that vervet monkeys, unique for their semantically rich alarm calls, have a fully developed inferior frontooccipital white matter pathway, suggesting a role for that tract in semantic conceptualization. In humans, the pMTG has been repeatedly shown to be involved in semantic learning and has been argued to function as a lexical interface (Rodríguez-Fornells et al., 2009; Gow, 2012), but the connectivity of this region has not been well-studied in other primates to determine whether its organization is unique to humans or shared with other species. Importantly, the pMTG serves as a cortical termination for an extensive number of white matter pathways from both dorsal and ventral streams (Turken \& Dronkers, 2011). In the present work, we explore how the pMTG system changed in the evolution by comparing white matter dissections of humans and chimpanzees.

\section{Methods}

Sample

High resolution diffusion-weighted imaging (DWI) data for 50 healthy subjects (mean age = $43.7 \pm 21.6$ yrs) were acquired by Janssen et al. (in prep). Diffusion-weighted data from 29 chimpanzees (Pan troglodytes; $28 \pm 17$ yrs), were obtained from a data archive of scans obtained prior to the 2015 implementation of U.S. Fish and Wildlife Service and National In- 
stitutes of Health regulations governing research with chimpanzees. Access to these scans was acquired through the US-based National Chimpanzee Brain Resource.

\section{Diffusion weighted imaging analyses}

For human participants, two binary masks were defined within the Montreal Neurological Institute (MNI) space using SPM Marsbar extraction tool and AAL anatomical atlas: pMTG and the anterior temporal lobe (ATL; both for the left hemisphere). The ATL was used as a second, potential semantic node, following the 'hub-and-spoke' model (Ralph, Jefferies, Patterson, \& Rogers, 2016). The pMTG mask was defined by restricting the middle temporal gyrus to its portion located posteriorly to the central sulcus ( $y=-18$, Turken \& Dronkers, 2011). The ATL mask was obtained by joining 5 parts: middle and superior temporal poles and the anterior portions of the inferior, middle and superior temporal gyri (terminating at $\mathrm{y}=-17$, thus not overlapping with pMTG). Subsequently, the masks were transferred to each individual's diffusion space and their corresponding white matter connections were calculated using a probabilistic approach (FSL probtrackx). All individual results were then warped to the MNI space and two unified outputs were calculated for the whole sample: the normalized mean, thresholded at $99 \%$ of the robust range, and the overlap of normalized and thresholded individual tractograms. In chimpanzees, masks were manually drawn to correspond to human cortical areas using homologous sulcal and gyral landmarks in chimpanzees, using recent sulcal/gyral maps for this species (Falk et al., 2018). All remaining steps were kept the same.

\section{Results}

Visual inspection of the results revealed an extensive ventral system of white matter pathways (including IFOF) originating from the left ATL seed in both humans and chimpanzees. Importantly, the maps did not substantially differ between the two species. In humans, the probabilistic tracking from pMTG showed that the ventral white-matter system extends to both the right hemisphere via the tapetum and to the dorsal pathways for language via the connection between the posterior superior temporal sulcus and the inferior parietal lobe. In chimpanzees, this circuitry was similar with regard to the interhemispheric connections, but connectivity to the dorsal stream was less robust than in humans. Formal quantification of these (dis)similarities is currently ongoing.

\section{Discussion}

Our results on the pMTG-related white-matter connections in humans confirmed the previous findings by Turken and Dronkers (2011). Furthermore, we extended the examination of these connections to another species, potentially confirming the uniqueness of the expansion towards the dorsal language stream in humans. Interestingly, the circuitry related to the ATL seed is similar between humans and chimpanzees, in both cases showing a connection with the IFOF. Together with the recent evidence on IFOF involvement in processing and integration of visual information for basic communication acts in vervet monkeys (Sarubbo et al., 2019), our results suggest that while the ATL/IFOF system may play a crucial role in conceptualization, it is the pMTG white matter circuitry that connects the ventral stream to the network related to phonological processing.

These findings also have important clinical implications. Our results add new evidence in support of the claim that the pMTG is a crucial node serving as a lexical interface. This implies that this region should be handled with particular caution in surgeries for removal of brain tumors or epileptic foci, and it could be key in understanding language recovery following brain injury. 


\section{References}

Falk, D., Zollikofer, C. P. E., Ponce de Leon, M., Semendeferi, K., Alatorre Warren, J. L., \& Hopkins, W. D. (2018). Identification of in vivo Sulci on the External Surface of Eight Adult Chimpanzee Brains: Implications for Interpreting Early Hominin Endocasts. Brain, Behavior and Evolution, 91(1), 45-58.

Gow D. W., Jr (2012). The cortical organization of lexical knowledge: a dual lexicon model of spoken language processing. Brain and language, 121(3), 273-288.

Hickok, G., \& Poeppel, D. (2007). The cortical organization of speech processing. Nature Reviews Neuroscience, 8(5), 393-402.

Janssen, N., Kessels, R.P.C., Mars, R.B., Llera, A., Beckmann, C.F., and Roelofs, A., Dissociating the functional roles of arcuate fasciculus subtracts in speech production (submitted).

Ralph, M. A. L., Jefferies, E., Patterson, K., \& Rogers, T. T. (2016). The neural and computational bases of semantic cognition. Nature Reviews Neuroscience, 18, 42.

Rodriguez-Fornells A., Cunillera T., Mestres-Misse A., de Diego-Balaguer R. (2009). Neurophysiological mechanisms involved in language learning in adults. Philos Trans $R$ Soc Lond B Biol Sci, 364 (1536), 3711-3735.

Roelofs, A., (2014). A dorsal-pathway account of aphasic language production: The WEAVER++ / ARC model. Cortex. 2014 Oct;59:33-48

Sarubbo, S., Petit, L., De Benedictis, A., Chioffi, F., Ptito, M., \& Dyrby, T. B. (2019). Uncovering the inferior fronto-occipital fascicle and its topological organization in nonhuman primates: the missing connection for language evolution. Brain Struct Funct.

Sierpowska, J., Gabarros, A., Fernandez-Coello, A., Camins, A., Castaner, S., Juncadella, M., François, C., Rodriguez-Fornells, A. (2019). White-matter pathways and semantic processing: intrasurgical and lesion-symptom mapping evidence. NeuroImage Clin, 22, 101704.

Turken, A. U., \& Dronkers, N. F. (2011). The neural architecture of the language comprehension network: converging evidence from lesion and connectivity analyses. Front SystNeurosci, 5, 1.

Wernicke, C. (1874). Der aphasiche Symptomenkomplex. Breslau: Cohn und Weigert. 


\title{
The Role of Arcuate Fasciculus on Severity of Language Impairments in Subcortical Stroke Patients
}

\author{
Jun Soo Noh, M.D. ${ }^{1}$, Sekwang Lee, M.D. ${ }^{3}$, Yoonhye Na, M.S. ${ }^{3}$, Minjae Cho, M.S. ${ }^{3}$, Yu Mi \\ Hwang, Ph.D. ${ }^{2}$, Woo-Suk Tae, Ph.D. ${ }^{2}$, Sung-Bom Pyun, M.D., Ph.D. ${ }^{1,2}$ \\ ${ }^{1}$ Department of Physical Medicine and Rehabilitation, Korea University College of Medicine, \\ Seoul, Republic of Korea \\ ${ }^{2}$ Brain Convergence Research Center, Korea University, Seoul, Republic of Korea \\ ${ }^{3}$ Department of Biomedical Sciences, Korea University, Seoul, Republic of Korea
}

\section{Introduction}

Subcortical aphasia is a form of aphasia that results from damage to subcortical regions such as the thalamus, internal capsule, and the basal ganglia. The exact mechanism of subcortical lesions causing aphasia is unclear, but several hypotheses were proposed; direct role of subcortical structures in language processing, diaschisis or hypoperfusion of cortical structures. Arcuate fasciculus (AF) is a major white matter tract connecting classic language center (Wernicke's area and Broca's area). Many studies investigated the relationship between AF damage and severity of aphasia in stroke patients, however there are few studies focusing on the patients with subcortical aphasia. In this study, we investigate the influence of AF damage on severity of subcortical aphasia after stroke using brain DTT analysis.

\section{Methods}

We collected the data of subcortical aphasia from the Stroke Outcome Prediction (STOP) database of Korea University Hospital and finally 41 patients who met the inclusion criteria were enrolled for this study. Inclusion criteria were 1) first-ever stroke, 2) supratentorial left hemispheric stroke, 3) right-handedness, 4) who performed aphasia evaluation and underwent diffusion tensor imaging (DTI). Also both patients with cerebral infarct or hemorrhages were included for subgroup analysis. Aphasia was evaluated by Korean version of Western Aphasia Battery (WAB), and aphasia quotient (AQ), fluency, comprehension, repetition and naming scores were used for analysis. The 50 decile of the AQ score was 61.6 points in the WAB test for Koreans26. Therefore, we clustered patients into two groups as a branch point of 61.6 points for either Good- or Poor- language function group. We reconstructed AF from DTI using DTIstudio software and extracted the fiber number (FN), average fiber length (FL), fractional anisotropy (FA), mean diffusivity (MD), radial diffusivity (RD) and axial diffusivity (AD). Also we classified left AF types in DTT: type A, no visualization of AF; type B, disrupted at the lesion; type C, preserved continuity. Also we measured lesion volume (LV) and volume of whole brain (BV) and we calculated percent LV (LV/BV x 100). A Spearman's correlation (r) was applied to assess relationship between AF parameters and aphasia scores and a classification and regression tree (CART) analysis was carried out to determine which factors best predict WAB. Also, subgroup CART analysis was performed according to the stroke type.

\section{Results}

Among the 41 patients with subcortical stroke, 33 subjects had hemorrhagic stroke and 8 patients were diagnosed as cerebral infarction. Thirty one out of 41 patients were diagnosed with aphasia; 12 anomic, 7 global and 5 Broca's aphasia. Mean duration between stroke onsets to examination was $27.39 \pm 10.58$ days in DTI and WAB in $13.44 \pm 6.85$ days.

All ischemic stroke patients have a lesion located in the basal ganglia. Ten out of 41 patients had a lesion restricted in a thalamus, and all of them were hemorrhagic strokes. All the left AF types in DTT were either type B or C. In the correlation analysis, LV was significantly 


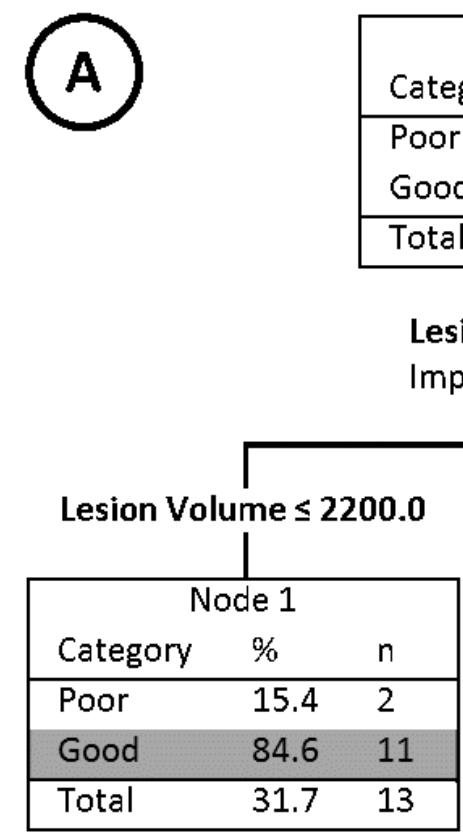

\begin{tabular}{|lll|}
\hline \multicolumn{3}{|c|}{ Node 0 } \\
Category & $\%$ & $\mathrm{n}$ \\
\hline Poor & 51.2 & 21 \\
Good & 48.8 & 20 \\
\hline Total & 100.0 & 41 \\
\hline \multicolumn{3}{|c|}{} \\
Lesion Volume $\left(\mathrm{mm}^{3}\right)$ \\
Improvement $=0.119$
\end{tabular}

ent $=0.119$

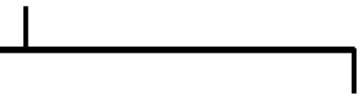

Lesion Volume $\mathbf{\leq} \mathbf{2 2 0 0 . 0}$
\begin{tabular}{|lll|}
\hline \multicolumn{3}{|c|}{ Node 1} \\
Category & $\%$ & $\mathrm{n}$ \\
\hline Poor & 15.4 & 2 \\
Good & 84.6 & 11 \\
\hline Total & 31.7 & 13 \\
\hline
\end{tabular}

Lesion Volume $>\mathbf{2 2 0 0 . 0}$

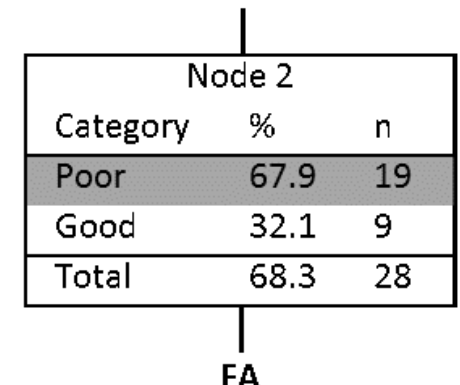

FA

Improvement $=0.047$

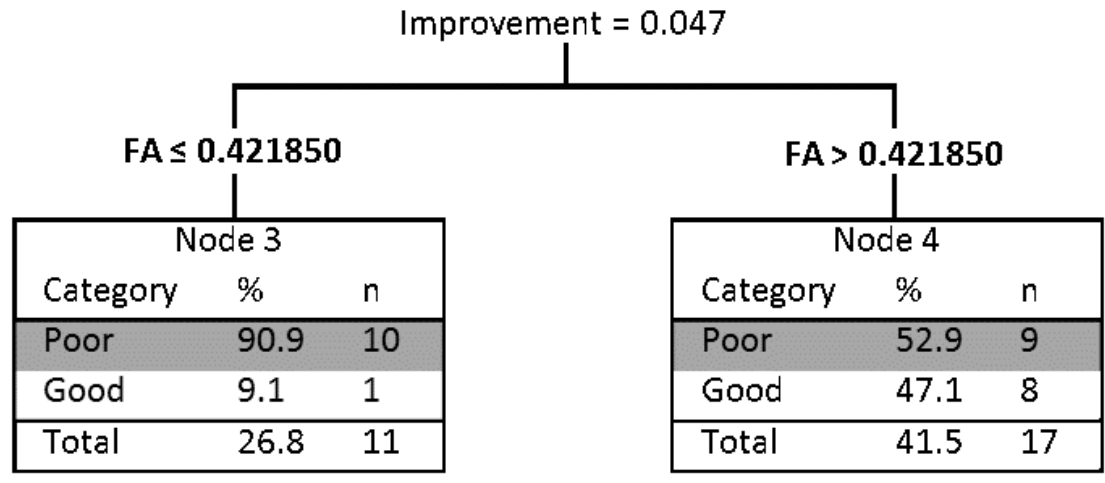

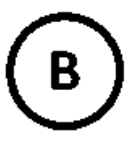

\begin{tabular}{|lll|}
\hline \multicolumn{3}{|c|}{ Node 0 } \\
Category & $\%$ & $\mathrm{n}$ \\
\hline Poor & 60.6 & 20 \\
Good & 39.4 & 13 \\
\hline Total & 100.0 & 33 \\
\hline & FA & \\
\hline
\end{tabular}

Improvement $=0.047$

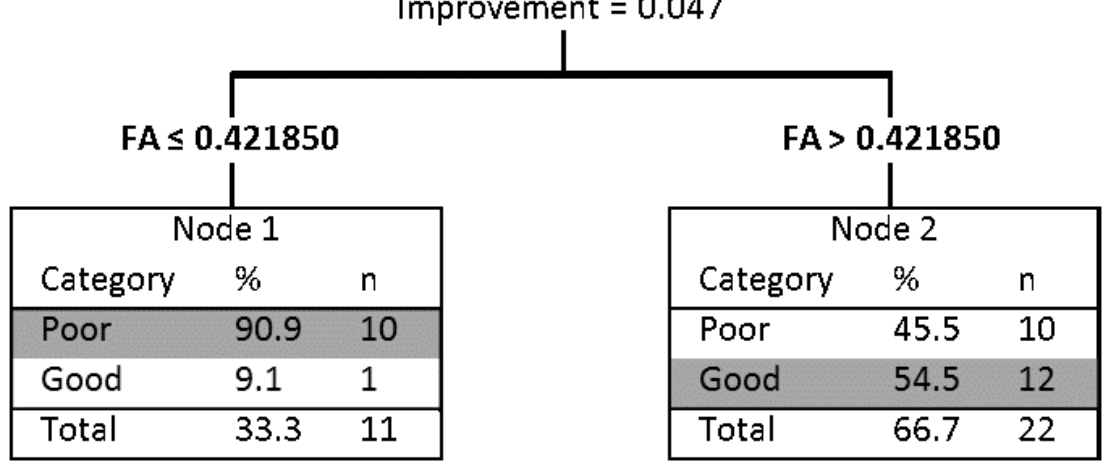

Figure 1. CART analyses of patients with language impairments after subcortical strokes. (A) Both hemorrhagic and ischemic stroke patients are included. (B) Only hemorrhagic stroke patients are included. 
associated with AQ and AQ subtests scores (fluency, repetition and naming). In AF, FA and MD were significantly associated with naming score. In subgroup analysis, the ischemic stroke group showed that FA, MD and AF type were significantly correlated with comprehension and naming scores. On the other hand, LV and LV per BV were associated with naming score in hemorrhagic stroke group.

The CART analysis produced a decision tree with lesion volume as the first decision point, followed by FA with a specificity of $90.5 \%$ and sensitivity of $55.0 \%$ (Fig. 1). The lesion volume of $2200 \mathrm{~mm} 3$ was identified as the point separates Good or Poor AQ scores. The CART analysis found that if patients had a lesion volume less than $2200 \mathrm{~mm} 3$ they were more likely to have a Good score. If patients with a lesion volume more than $2200 \mathrm{~mm} 3$ have FA less than 0.421850 , they were highly likely to have a Poor score. As a subgroup analysis, alternative CART analyses were carried out for hemorrhagic stroke patients. If ischemic stroke patients were removed, the CART analysis selected FA to predict WAB score for patients first; specificity of $50.0 \%$ and sensitivity of $92.3 \%$. The FA cut-off point was the same (0.421850). If patients with a FA lower than 0.421850 , they were highly likely to have a Poor outcome (10 out of 11 patients).

\section{Discussion}

The primary aim of this study was to investigate the relationship between AF parameters and aphasia severity in patients with subcortical stroke. To our best knowledge, this is the first study to demonstrate an association between aphasia severity and neuroimaging factors using DTI in subcortical stroke. In our study, FA and MD showed a significant correlation with AQ and naming scores and these findings coincides well with previous reports in post-stroke subcortical aphasia. In CART analysis, LV and FA value was a significant predictor of subcortical aphasia. Previously proposed hypotheses, such as direct role of subcortical structure on language, diaschisis, hypoperfusion or hypometabolism, can still be accepted, while damage of AF is also an important underlying mechanism of subcortical aphasia. And its contribution seems to be different according to infarction and hemorrhagic stroke. In subgroup analysis, FA was the only significant variable that can predict language impairment in the hemorrhagic stroke. It can be suggested that amount of AF damage is more important than LV in subcortical aphasia after hemorrhagic stroke. Considering significant correlations between DTI values (FA, $\mathrm{MD}, \mathrm{AF}$ type) and aphasia severity (fluency, comprehension) were seen only in the ischemic stroke group, it can be assumed that the integrity of AF also has a crucial role in patients with cerebral infarction.

\section{References}

Kang EK, Sohn HM, Han MK, et al. Subcortical Aphasia After Stroke. Ann Rehabil Med 2017;41:725-33.

Nadeau SE, Crosson B. Subcortical aphasia. Brain Lang 1997;58:355-402; discussion 18-23.

Bitar A, Fohanno D, Denvil D, et al. Intraoperative mapping of the subcortical language pathways using direct stimulations: An anatomo-functional study. Brain 002;125:199214.

Demeurisse G, de Beuckelaer R, Derouck M, et al. Subcortical aphasia: a specific clinical picture? Eur J Neurol 1995;2:317-23.

Murdoch BE. Subcortical brain mechanisms in speech and language. Folia Phoniatrica Et Logopaedica 2001;53:233-51.

Kennedy M, Murdoch BE. Chronic Aphasia Subsequent to Striato-capsular and Thalamic Lesions in the Left Hemisphere. Brain and Language 1993;44:284-95.

Hoffmann M, Chen R. The spectrum of aphasia subtypes and etiology in subacute stroke. $J$ Stroke Cerebrovasc Dis 2013;22:1385-92. 
Granadillo ED, Arciniegas DB. Poststroke subcortical aphasia and neurobehavioral disturbances without motor or sensory deficits. J Neuropsychiatry Clin Neurosci 2015;27:e1657.

Crosson B. Subcortical mechanisms in language: lexical-semantic mechanisms and the thalamus. Brain Cogn 1999;40:414-38.

Crosson B. Thalamic mechanisms in language: a reconsideration based on recent findings and concepts. Brain Lang 2013;126:73-88.

Llano DA. Functional imaging of the thalamus in language. Brain Lang 2013;126:62-72.

Sebastian R, Schein MG, Davis C, et al. Aphasia or Neglect after Thalamic Stroke: The Various Ways They may be Related to Cortical Hypoperfusion. Front Neurol 2014;5:231.

Osawa A, Maeshima S. Aphasia and unilateral spatial neglect due to acute thalamic hemorrhage: clinical correlations and outcomes. Neurological Sciences 2016;37:565-72.

Maeshima S, Osawa A. Thalamic Lesions and Aphasia or Neglect. Curr Neurol Neurosci Rep 2018;18:39.

Damasio AR, Damasio H, Rizzo M, et al. Aphasia with nonhemorrhagic lesions in the basal ganglia and internal capsule. Arch Neurol 1982;39:15-24.

Radanovic M, Mansur LL. Aphasia in vascular lesions of the basal ganglia: A comprehensive review. Brain and Language 2017;173:20-32.

Choi JY, Lee KH, Na DL, et al. Subcortical aphasia after striatocapsular infarction: quantitative analysis of brain perfusion SPECT using statistical parametric mapping and a statistical probabilistic anatomic map. J Nucl Med 2007;48:194-200.

Ishii R, Takeuchi S, Ohsugi S, et al. [Cerebral blood flow in patients with putaminal hemorrhage. Using 133Xe inhalation method]. Neurol Med Chir (Tokyo) 1982;22:813-21.

Perani D, Vallar G, Cappa S, et al. Aphasia and neglect after subcortical stroke. A clinical/cerebral perfusion correlation study. Brain 1987;110 ( Pt 5):1211-29.

Kim SH, Jang SH. Prediction of Aphasia Outcome Using Diffusion Tensor Tractography for Arcuate Fasciculus in Stroke. American Journal of Neuroradiology 2013;34:785-90.

Geva S, Correia M, Warburton EA. Diffusion tensor imaging in the study of language and aphasia. Aphasiology 2011;25:543-58.

Hosomi A, Nagakane Y, Yamada K, et al. Assessment of arcuate fasciculus with diffusiontensor tractography may predict the prognosis of aphasia in patients with left middle cerebral artery infarcts. Neuroradiology 2009;51:549-55.

Ivanova MV, Isaev DY, Dragoy OV, et al. Diffusion-tensor imaging of major white matter tracts and their role in language processing in aphasia. Cortex 2016;85:165-81.

Hosomi A, Nagakane Y, Yamada K, et al. Assessment of arcuate fasciculus with diffusiontensor tractography may predict the prognosis of aphasia in patients with left middle cerebral artery infarcts. Neuroradiology 2009;51:549-55.

Shewan CM, Kertesz A. Reliability and Validity Characteristics of the Western Aphasia Battery (Wab). Journal of Speech and Hearing Disorders 1980;45:308-24.

Kim H, Na DL. Normative data on the Korean version of the Western Aphasia Battery. Journal of Clinical and Experimental Neuropsychology 2004;26:1011-20.

Jiang H, van Zijl PC, Kim J, et al. DtiStudio: resource program for diffusion tensor computation and fiber bundle tracking. Comput Methods Programs Biomed 2006;81:106-16.

Nucifora PG, Verma R, Melhem ER, et al. Leftward asymmetry in relative fiber density of the arcuate fasciculus. Neuroreport 2005;16:791-4.

Vernooij MW, Smits M, Wielopolski PA, et al. Fiber density asymmetry of the arcuate fasciculus in relation to functional hemispheric language lateralization in both right- and left-handed healthy subjects: a combined fMRI and DTI study. Neuroimage 2007;35:106476.

Wheeler-Kingshott CAM, Cercignani M. About "Axial" and "Radial" Diffusivities. Magnetic 
Resonance in Medicine 2009;61:1255-60.

Jang SH. Diffusion tensor imaging studies on arcuate fasciculus in stroke patients: a review. Frontiers in Human Neuroscience 2013;7.

Forkel SJ, Thiebaut De Schotten M, Dell'Acqua F, et al. Anatomical predictors of aphasia recovery: a tractography study of bilateral perisylvian language networks. Brain 2014;137:2027-39.

Rosso C, Vargas P, Valabregue R, et al. Aphasia Severity in Chronic Stroke Patients: A Combined Disconnection in the Dorsal and Ventral Language Pathways. Neurorehabilitation and Neural Repair 2015;29:287-95.

Hillis AE, Barker PB, Wityk RJ, et al. Variability in subcortical aphasia is due to variable sites of cortical hypoperfusion. Brain and Language 2004;89:524-30.

Kirk A, Kertesz A. Cortical and Subcortical Aphasias Compared. Aphasiology 1994;8:65-82. 


\title{
White-matter bottleneck in small vessel disease: A lesion-symptom mapping study of executive-language functions.
}

\author{
Ileana L. Camerino ${ }^{2}$, Joanna Sierpowska ${ }^{2,3}$, Nathalie H. Meyer ${ }^{2}$, Anil M. Tuladhar ${ }^{1}$, Roy P.C. \\ Kessels $^{2,3}$, Frank-Erik de Leeuw ${ }^{1}$, and Vitória Piai ${ }^{2,3}$ \\ ${ }^{1}$ Donders Institute for Brain, Cognition and Behaviour, Centre for Neuroscience, Department \\ of Neurology, Radboud University, Nijmegen, The Netherlands \\ ${ }^{2}$ Donders Institute for Brain, Cognition, and Behaviour, Donders Centre for Cognition, \\ Radboud University, Nijmegen, The Netherlands \\ ${ }^{3}$ Department of Medical Psychology, Radboud University Medical Center, Nijmegen, The \\ Netherlands
}

\section{Introduction}

Cerebral small vessel disease (CSVD), characterized by the presence of white matter lesions (WML), is among the main causes of vascular cognitive impairment. The most well-studied cognitive domains showing impairment in CSVD are executive functioning and processing speed, which are correlated with total WML volume 1,2. By contrast, the domain of language has received much less attention 3,4. Recent studies indicate that WML location might be more informative than total WML volume in explaining the cognitive profile of CSVD 5. However, these studies only investigated tasks of executive function and processing speed, whereas other brain functions that might be more dependent on WML location, such as language, have remained understudied 6-8. In addition, these studies used global compound scores of executive function and processing speed with and without language involvement (5), precluding inferences regarding whether there is a core network underlying executive and language tasks. The present study investigates whether WML location is associated with poorer performance in executive-language tasks, as analyzed at a single task level.

\section{Methods}

Study population and Neuropsychological assessments

This study included a cohort of 445 CSVD patients without dementia, with varying burden of WML. WML were defined as hyperintense lesions on FLAIR MRI without corresponding cerebrospinal fluid-like hypointense lesions on the T1 weighted image. The WML were segmented on FLAIR images and transformed into Montreal Neurological Institute 152 (MNI) standard space. The Stroop (word reading, color naming, and color-word naming) and the verbal fluency tests were used as measures of language production with varying degrees of executive demands. The digit symbol modality (DSMT) was used as a control task as it does not require verbal abilities.

\section{Voxel lesion symptom mapping analysis}

A voxel-based lesion symptom mapping (VLSM) approach 9 was used. In this approach, a t-test is performed at every voxel, comparing test scores (verbal fluency, each of the three tasks of the Stroop test and DSMT) in individuals with and without a WML in each voxel. Analyses were limited to those voxels where at least $4 \%(\mathrm{~N}=18)$ of the individuals had a lesion with the goal of minimizing biased parameter estimates. To correct for multiple comparison, permutation testing was used. The cut-off for a significant cluster size was determined based on 6000 iterations, with a voxel-wise threshold set at an alpha level of 0.0510 . All VLSM analyses were corrected for age, gender, education, and lesion size. Additionally, to control for the processing speed component in language-related tasks, all VLSM analyses (verbal fluency, color-word naming and DSMT) were further corrected by a "processing speed" score. This was obtained by averaging the score of word reading and color naming of the Stroop test. Then, 
we divided the scores of verbal fluency, color-word naming test and DSTM by the processing speed score.

\section{Results}

The VLSM analyses revealed statistically significant clusters for verbal fluency, and Stroop word reading, color naming and color-word naming, but not for DSMT. Worse scores in all tests were associated with WML predominantly in the forceps minor, bilateral thalamic radiations and the caudate nuclei. This set of brain areas was similar across all tests. The lesion-symptom associations remained the same once the scores of the verbal fluency and Stroop color-word naming tests were corrected for processing speed.

\section{Discussion}

A relationship was found between WML in a core fronto-striatal network and executivelanguage functioning in CSVD independent of lesion size. This circuitry formed by the caudate nuclei, forceps minor and thalamic radiations, seems to underlie executive-language functioning beyond the role of general processing speed and it might constitute a bottleneck area in CSVD. Finally, the contribution of this circuitry seems to be stronger for tasks requiring language functioning.

\section{References}

Prins, N.D. Van Dijk, E.J. Den Heijer, T. et al. (2005). Cerebral small-vessel disease and decline in information processing speed, executive function and memory. Brain, 128, 9, 2034-2041.

Wardlaw, J.M. Smith, C. Dichgans, M. (2013). Mechanisms of sporadic cerebral small vessel disease: Insights from neuroimaging. Lancet Neurol, 12, 5, 483-497.

Welker, K.M. De Jesus, R.O. Watson, R.E. Machulda, M.M. Jack, C.R. (2012). Altered Functional MR Imaging Language Activation in Elderly Individuals with Cerebral Leukoaraiosis. Radiology, 265,1, 222-232.

Herbert, V. Brookes, R.L. Markus, H.S. Morris, R.G.(2014). Verbal fluency in cerebral small vessel disease and alzheimer's disease. J Int Neuropsychol Soc., 20, 4, 413-421.

Biesbroek, J.M. Weaver, N.A. Biessels, G.J. (2017). Lesion location and cognitive impact of cerebral small vessel disease. Clin Sci., 131, 8, 715-728.

Biesbroek, J.M. Weaver, N.A. Hilal, S. et al. (2016). Impact of Strategically Located White Matter Hyperintensities on Cognition in Memory Clinic Patients with Small Vessel Disease. PLoS One, 11, 11, 1-17.

Duering, M. Gonik, M. Malik, R. et al. (2013). Identification of a strategic brain network underlying processing speed deficits in vascular cognitive impairment. Neuroimage, 66, 177-183.

Smith, E.E. Jeng, J. Fisch, l. B. Blacker, D. (2011). Correlations between MRI white matter lesion location and executive function and episodic memory. Neurology, 76, 1492-1499.

Bates, E. Wilson, S.M. Saygin, A.P. et al. (2003). Voxel-based lesion-symptom mapping. Nat Neurosci., 6, 5, 448-450.

Kimberg, D.Y. Coslett, H.B. Schwartz, M.F. (2007). Power in Voxel-based Lesion -Symptom Mapping. J Cogn Neurosci., 19, 1067-1080. 


\title{
CForests in the trees: Using conditional inference random forests on MEG data to explore 'what fires together' in a picture naming task
}

\author{
Elissa Asp ${ }^{1}$, Antoine Tremblay ${ }^{2}$, Graham Flick $^{3}$, Aaron Newman ${ }^{2,4}$ \\ ${ }^{1}$ English and Linguistics, Saint Mary's University, Halifax, NS, Canada \\ ${ }^{2}$ Neurocognitive Imaging Lab, Dalhousie University, Halifax, NS \\ ${ }^{3}$ Department of Psychology, New York University, New York, NY, USA \\ ${ }^{4}$ Department of Psychology and Neuroscience, Dalhousie University, Halifax, NS, Canada
}

\section{Introduction}

Studies of semantic variant primary progressive aphasia (sPPA) suggest a role for the lateral anterior temporal lobes (ATL), including the temporal poles (TP), in semantic processing. Because of the strength of association between semantic impairments and ATL damage, there is also widespread agreement that the ATLs function as some sort of semantic hub. However, there are competing proposals as to the nature of this semantic hub. There is the 'amodal semantic hub' view, according to which the ATLs support semantic integration or convergence because they host amodal semantic representations (e.g. Lambon Ralph et al. 2017).

In contrast, Mesulam and colleagues (e.g. 2013; 2015a) have argued that the left ATL is specifically part of the language network. This argument is supported by evidence from individuals with atrophy mostly limited to the left ATL/temporal pole (TP), who have prominent naming and categorization deficits but comparatively intact object recognition abilities, whereas individuals with more widely distributed and bilateral atrophy are also profoundly impaired on object recognition tasks (Mesulam et al. 2013, 2015; also e.g. Binney et al. 2016; Luzzi et al. 2017; Snowdon et al. 2017).

Work on language processing in healthy groups also supports the idea of (sub-regions of) the left ATL contributing to morphological and lexical retrieval or selection processes (e.g. Lau et al. 2013; Lewis et al. 2011) and semantic combinatorial processes for phrases and complex words (e.g. Flick et al. 2018; Westerlund et al. 2015). In short, there is good evidence for the left ATL being involved in various aspects of semantic and lexical processing but a variety of proposals as to the nature of that involvement.

Such functional variability raises the possibility of multiple networks with nodes in temporal cortex, which may contribute to different components of language tasks. To approach this, we recorded magnetoencephalography (MEG) data while participants performed a picture-naming task. Data were analyzed using the combination of signal space separation beamformer (Vrba et al., 2010) and conditional inference random forest (CForest; Horthorn et al., 2006) methods. CForest generated predicted time-courses from sources, which were then clustered according to their temporal covariance, yielding temporally co-varying networks of cortical sources (Tremblay et al. In prep.). Here we focus on clusters with sources localized to ATL.

\section{Methods}

In Tremblay et al. (In Prep) fourteen healthy adults sequentially named 200 colour pictures (Rossion \& Pourtois, 2004) while continuous MEG recordings were collected. The un-averaged sensor data was projected onto the cortex using a Signal Space Separation Beamformer (Vrba et al., 2010) with 278 virtual electrodes (VEs) from the parcellation of Shen et al. (2013). CForest generated predicted time-courses at each discrete VE from a model predicting amplitude as a function of time and $\mathrm{x}-, \mathrm{y}-$, z-coordinates. Predicted VE time-courses were clustered together in a step-wise manner, from 97.5 to $82.5 \%$ covariance. Areas with covariance below $82.5 \%$ 
were treated as 'unclustered'. No thresholds were set for amplitude fluctuations or cluster size. For the present work, we extracted clusters with nodes in the temporal lobes and report the structure and time-courses of those with ATL nodes.

\section{Results}

\section{Cluster structures}

The clustering analysis produced 24 clusters with areas in temporal cortex. Five clusters had areas in ATL. These (plus C21) are listed in Table 1.

Table 1. Cluster areas

\begin{tabular}{lllll}
\hline Cluster \# & Temporal & BA & MNI $(\mathrm{x}, \mathrm{y}, \mathrm{z})$ & Other areas \\
\hline 2 & IITGa $^{*}$ & 20 & $-49,-10,-32$ & rCrus I, rCrus II, rTOFus, rVI \\
17 & lTP* $^{*}$ & 38 & $-41,10,-30$ & 1Accumbens, rCingulateGp, lFOrbital*, ISubcallosal \\
& ITP* $^{*}$ & 38 & $-26,4,-37$ & \\
21 & lPHGa $^{*}$ & 53 & $-26,-4,-18$ & \\
25 & lMTGp* & 21 & $-57,-12,-13$ & lHippocampus*, lTFusC,p* \\
& rITGa & 20 & $46,1,-34$ & rAmygdala, rFOrbital, rFPole, lVI* \\
33 & rTP & 38 & $37,14,-28$ & \\
52 & rPP & 22 & $57,0,2$ & rInsula \\
\hline
\end{tabular}

Legend: a=anterior, $\mathrm{C}=$ cortex, $\mathrm{F}=$ frontal, Fus=fusiform, $\mathrm{I}=$ inferior, $\mathrm{G}=$ gyrus, $\mathrm{M}=$ middle, $\mathrm{O}=$ occipital, $\mathrm{PH}=$ parahippocampal, $\mathrm{P} p=$ Planum Polar, $p=$ Pole, $p=$ posterior, $\mathrm{T}=$ temporal; areas with $*$ become active $40 \mathrm{~ms}$.

\section{Timing and predicted activity}

Overall, the data show widespread bilateral activity that began in primary visual cortex. Mean activity was greater in the right than the left hemisphere, with the largest amplitudes mainly restricted to areas in left temporal and frontal clusters, as well as brain stem and cerebellum. However, consistent with Llorens et al.'s (2014) findings for sequential naming tasks, naming took longer than in blocked designs (median voice onset $1000 \mathrm{~ms}$ ) and very early engaged anterior areas in the left lateral cortex, as well as medial frontal and inferior temporal areas. Indeed, these left frontal and temporal areas showed minor divergences from their baselines at $40 \mathrm{~ms}$ and large peaks around $120-150 \mathrm{~ms}$ and $250 \mathrm{~ms}$, with further increases around 400 and $600 \mathrm{~ms}$. Homologous right hemisphere clusters became active later ( $100 \mathrm{~ms})$ and did not show comparable magnitudes.

\section{Discussion}

We investigated the use of CForest to characterize temporally-covarying networks of cortical sources during a picture-naming task. Compared to other methods, CForest has the advantages of being able to handle large numbers of predictor variables and, in the context of brain data analysis, allows one to treat time and space as continuous variables rather than discretizing. This avoids the assumption of stationarity in functional connectivity analyses. Clustering the predicted time-courses from CForest by their covariance produced clusters that are plausible as 'functional networks' based on previous literature. To cite one example, TP areas clustered with frontal orbital cortex in each hemisphere. However, the left hemisphere TP cluster (C17) was active earlier and much more strongly across the time course than the homologous right hemisphere cluster (C25). C17's activity in the early time course is paralleled only by a frontal cluster (C16) consisting of polar, orbital, and medial frontal areas. Speculatively, the early activation of C17 may be related to 'control-like' processes, beginning almost concurrently with primary visual responses. Further, the fact that C17 is active across the entire time course, with more than one peak, suggests that it could be involved in the selection, combination, or integration of more than one type of information. Its activity and architecture would also 
explain performance impairments in early sPPA, given atrophy and increased diffusivity along the uncinate pathway from TP to OFC (Catani et al. 2013).

\section{References}

Binney, R. J., Henry, M. L., Babiak, M., Pressman, P. S., Santos-Santos, M. A., Narvid, J., ... Gorno-Tempini, M. L. (2016). Reading words and other people: A comparison of exception word, familiar face and affect processing in the left and right temporal variants of primary progressive aphasia. Cortex, 82, 147-163.

Catani, M., Mesulam, M. M., Jakobsen, E., Malik, F., Martersteck, A., Wieneke, C., ... Rogalski, E. (2013). A novel frontal pathway underlies verbal fluency in primary progressive aphasia. Brain, 136(Pt 8), 2619-2628.

Flick, G., Oseki, Y., Kaczmarek, A.R., Al Kaabi, M., Marantz, A., Pylkkänen. 2018. Building words and phrases in the left temporal lobe. Cortex, 106: 213-236.

Hothorn, T., Hornik, K., \& Zeileis, A. (2006). Unbiased recursive partitioning: A conditional inference framework. Journal of Computational and Graphical statistics, 15(3), 651-674.

Lambon Ralph, M.A., Jeffries, E., Patterson, K., Rogers, T.T. (2017). The neural and computational basis of semantic cognition. Nature Reviews: Neuroscience. 18(1):42-55.

Llorens, A., Trébuchon, A., Riès, S., Liégeois-Chauvel, C., \& Alario, F. X. (2014). How familiarization and repetition modulate the picture naming network. Brain and Language, 133, 47-58.

Luzzi, S., Baldinelli, S., Ranaldi, V., Fabi, K., Cafazzo, V., Fringuelli, F., Silvestrini, M., Provinciali, L., Reverberi, C., Gainotti, G. 2017. Famous faces and voices: Differential profiles in early right and left semantic dementia and in Alzheimer's disease, Neuropsychologia, 94, 118-128.

Mesulam, M-M., Wieneke, C., Hurley, R., Rademaker, A., Thompson, C.K., Weintraub, S., Rogalski, E.J. 2013. Words and objects at the tip of the left temporal lobe in primary progressive aphasia, Brain, 136, 601-618.

Mesulam, M-M., Rogalski, E. J., Wieneke, C., Hurley, R. S., Geula, C., Bigio, E. H., ... \& Weintraub, S. (2015). Primary progressive aphasia and the evolving neurology of the language network. Nature Reviews Neurology, 10(10): 554-569.

Rossion, B., \&Pourtois, G. (2004). Revisiting Snodgrass and Vanderwart's object pictorial set: The role of surface detail in basic-level object recognition. Perception, 33(2), 217-236.

Shen, X., Tokoglu, F., Papademetris, X., \& Constable, R. T. (2013). Groupwise whole-brain parcellation from resting-state fMRI data for network node identification. Neuroimage, 82: $403-415$.

Tremblay, A., Flick, G., Asp., E., \& Newman, A.J. In preparation. MEG spatio-temporal dynamics of simple picture naming.

Tremblay, A., Asp, E., Johnson, A., Bardouille, T., Newman, A.J. 2016. What the networks tell us about serial and parallel processing: An MEG study of language networks in an overt picture description task. The Mental Lexicon, 11.1: 115-160.

Vrba, J., Taulu, S., Nenonen, J., \& Ahonen, A. (2010). Signal space separation beamformer. Brain topography, 23(2), 128-133.

Westerlund, M., Kastner, I., Al Kaabi, M., \& Pylkkänen, L. (2015). The LATL as locus of composition: MEG evidence from English and Arabic. Brain and Language, 141, 124134 . 


\title{
What are the active ingredients in anomia therapy? A random forest analysis of anomia research from 2009-2018
}

\author{
Wei Ping SZE ${ }^{1}$, Jane WARREN ${ }^{1}$, Solène HAMEAU ${ }^{2}$, Wendy BEST ${ }^{1}$ \\ ${ }^{1}$ University College London \\ ${ }^{2}$ Macquarie University
}

\section{Introduction}

Anomia often occurs in adults with spoken expressive aphasia (Murdoch, 1990). Despite the wealth of research, the precise ingredients that explain successful word-finding outcomes in spoken anomia therapy remain unclear. Often, different combinations of therapy components are applied in research and clinical practice, masking the active ingredient(s) responsible for treatment efficacy. For example, to treat an impairment in accessing the phonological output lexicon, researchers have suggested having participants generate phonological cues (e.g., Leonard, Rochon, \& Laird, 2008). Others have proposed the use of progressive cues, primarily either phonological or orthographic information (e.g., Hickin, Best, Herbert, Howard, \& Osbourne, 2001). This diversity in treatment methods can unfortunately be confusing for a speech and language therapist. This study therefore adopts a novel approach: It systematically searched and combined individual data (obtained from group studies, case series or case studies), before employing random forest to determine the active ingredients in spoken single-word therapy.

\section{Methods}

Systematic search

A comprehensive search was conducted using 17 electronic databases, including general search engines (e.g., PubMed, PsychINFO) and more speech-therapy related ones (e.g., AMED for allied health professionals). After removing duplicated entries, the first and third authors independently reviewed and screened over 3,900 entries, based on pre-specified criteria. The primary selection criteria include: (1) Studies must be spoken single-word naming therapies; (2) participants are adults with word-finding difficulties as part of aphasia after stroke; (3) therapies are language-based approaches, i.e., semantic, orthographical and/or phonological approaches; (4) the reports must also be written in English and (5) include original data. We did not include studies investigating total communication and therapies beyond single-word naming, like the use of phrases to exchange information (e.g., Pulvermüller et al., 2001).

From the suitable studies, individual data were obtained. The final dataset was made up of data from just over 220 individuals. Information on therapy components as well as four outcome measures (short-term outcome for treated and untreated items respectively, long-term outcome for treated and untreated items respectively) were extracted. Short-term is defined as three weeks or less. Long-term is defined as more than three weeks after intervention. We sought to be comprehensive when accounting for the information on therapy components. The therapy components are thus divided into four groups: (1) Information on the therapy regimen (e.g., frequency of sessions per week); (2) Information on the words used in treatment (e.g., how many items were treated across sessions); (3) Information about the techniques (e.g., Were phonological cues used? Were semantic tasks used?); and (4) Information about the application of techniques (e.g., Were the cues applied in an increasing or decreasing order?). All these pieces of information were then entered as variables into the meta-analysis.

\section{Meta-analysis}

Random forest was used to compute the meta-analysis. Random forest is an established ensemble learning method useful for determining variables and has been fruitfully applied across 
various disciplines (e.g., de Aguiar, Bastiaanse, \& Miceli, 2016; Stephan, Stegle, \& Beyer, 2015). It does so by imputing classification and regression decision trees (Breiman, 2000). Random forest is appropriate due to the mixture of categorical and continuous variables-of-interest, the 'large- $p$ small- $n$ ' characteristic of our data, as well as its versatility in managing data derived from small- $n$ design studies.

\section{Results}

Concordance statistics and the "Out Of Bag" (OOB) errors obtained for the random forests suggested that the imputed random forest models were accurate (i.e., concordance indices were greater than '0.83' and OOB rates were less than '0.18' for all imputed models). The primary results were based on the variables' order of importance when predicting a particular outcome. For the successful naming of treated items in the short term, the results suggested that providing the written form of the target word, explicit application of orthographic part-word cues, and applying cues based on responses were important variables. For successful naming of treated items in the long-term phase, feedback on naming accuracy, providing written form of the target word and explicit application of orthographic part-word cues were important variables. As for the naming of untreated items in the short term, providing the written word form of the target word was also important.

\section{Discussion}

From data extracted from over 220 individuals with aphasia, the role of orthography appeared to be important across the three outcome measures, whether the use of orthography was operationalized in terms of the provision of written target word or as orthographic part-word cues. We will discuss the clinical implications of maximising ingredients that drive successful naming rehabilitation, particularly in relation to the application of orthography. We will also briefly discuss the importance of using advanced statistical approaches to integrate findings from small-n research data, in order to advance the field.

\section{References}

Breiman, L. (2000). Random forests. Machine Learning, 45(1), 5-32. doi: 10.1023/A:1010933404324

de Aguiar, V., Bastiaanse, R., \& Miceli, G. (2016). Improving production of treated and untreated verbs in aphasia: A meta-analysis. Frontiers in Human Neuroscience, 10(468). doi: $10.3389 /$ fnhum.2016.00468

Hickin, J., Best, W., Herbert, R., Howard, D., \& Osborne, F. (2002). Phonological therapy for word-finding difficulties: A re-evaluation. Aphasiology, 16(10-11), 981-999. doi: 10.1080/02687030244000509

Leonard, C., Rochon, E., \& Laird, L. (2008). Treating naming impairments in aphasia: Findings from a phonological components analysis treatment. Aphasiology, 22(9), 923947. doi: 10.1080/02687030701831474

Murdoch, B. E. (1990). Acquired speech and language disorders: A neuroanatomical and functional neurological approach. London, UK: Chapman and Hall.

Pulvermüller, F., Neininger, B., Elbert, T., Mohr, B., Rockstroh, B., Koebbel, P., \& Taub, E. (2001). Constraint-induced therapy of chronic aphasia after stroke. Stroke, 32(7), 1621-1626. doi: 10.1161/01.STR.32.7.1621

Stephan, J., Stegle, O., \& Beyer, A. (2015). A random forest approach to capture genetic effects in the presence of population structure. Nature Communications, 6, 7432. doi: $10.1038 /$ ncomms 8432 


\title{
Neural substrates associated with the recovery of verb anomia: the role of sensorimotor strategies in the improvement of naming abilities in aphasia
}

\author{
Edith Durand ${ }^{1,2}$, Pierre Berroir ${ }^{1,3}$ and Ana Inés Ansaldo ${ }^{1,2}$ \\ ${ }^{1}$ Centre de recherche de l'institut universitaire de Gériatrie de Montréal CRIUGM \\ ${ }^{2}$ École d'orthophonie, Faculté de Médecine, Université de Montréal \\ ${ }^{3}$ Ecole Polytechnique, Université de Montréal
}

\section{Introduction}

In the context of aphasia, verb anomia is more frequent than noun deficits (Mätzig et al., 2009), and their negative impact on daily communication abilities has been well documented (Rofes, Capasso, \& Miceli, 2015). However, most research on anomia has focused on noun retrieval, whereas therapies targeting verb anomia remain scarce (Webster \& Whitworth, 2012). This is particularly surprising, considering the central role of verbs in sentence and speech production (Conroy, Sage, \& Lambon Ralph, 2006). Verb therapy, irrespective of whether verbs are treated within a single-word or sentence context, is effective in improving the retrieval of treated verbs but with limited generalization to untreated verbs (Webster \& Whitworth, 2012). Amongst single-word retrieval therapies, therapies based on sensorimotor strategies have been studied, but rarely explored with neuroimaging techniques. The present study reports on the efficacy of Personalized Observation, Execution, and Mental Imagery (POEM) therapy, a new approach that integrates sensorimotor and language-based strategies to treat verb anomia, within a structured therapy protocol based on principles of experience-dependent neuroplasticity (Durand et al. in prep).

\section{Methods}

\section{Participants}

Ten participants with chronic aphasia and verb anomia were recruited for the study and two of them were also followed up in a pre/post-therapy fMRI study. Participants presented a single lesion in the left hemisphere, varying in size and location, and all of the were diagnosed with non-fluent aphasia, and verb anomia ranging from $21 \%$ to $94 \%$ of degree of severity. They were chronic (time post onset ranging from 2 to 34 years).

\section{Experimental procedure}

Background assessment

Language and cognitive function tests were completed at baseline. Language tests included comprehension, naming, repetition and fluency to allow a complete description of the aphasia profile. Cognitive function tests included short term memory and executive functions tests to obtain a cognitive background.

\section{Stimuli}

A personalized set of stimuli was built for each participant based on the naming performance on baseline, to build a treated and a non-treated set of stimuli, controlled for linguistic variables.

\section{POEM therapy}

POEM was administered according to a strict protocol (Durand et al. in prep) and in a massed stimulation schedule of three one hour-sessions per week, over five weeks. During each session, participants were trained to name actions presented in 5 -second videos. If the participant could not name the action within 5 to $10 \mathrm{~s}$, he was asked to make the gesture associated with this action, helped by the SLP. If he could not name the action, the participant was asked to imagine 
the action in a personal context. If the action is still not named, the SLP asked to close eyes and imagine the action in a relevant context. After these prompts, the word was given to the participant, who was asked to repeat it once.

\section{fMRI protocol}

The two participants who participated to the fMRI study underwent an initial fMRI session (T1), which identified the neural substrate of spontaneous correct naming. Afterward, these two participants received POEM. A second fMRI session (T2) was performed after five weeks of therapy. This session allowed us to identify the brain areas that subserved therapy-induced neuroplasticity. During both fMRI sessions, patients performed an overt naming task.

\section{fMRI design and parameters}

Images were acquired using a 3T MRI Siemens Trio scanner with a standard 32-channel head coil. All the parameters are presented in Durand et al, 2018. Responses to the fMRI naming task were recorded and coded offline. Preprocessing and statistical analyses were performed using SPM12 software. Neuroimaging data analyses were performed only on correct responses. Individual activation maps were calculated for each condition for the whole brain with cluster size superior to 10 voxels and $p<.001$ uncorrected. Furthermore, we are currently processing functional connectivity analysis following the same procedure as Berroir et al., 2017. Our goal is to examine the integration changes in a pre-post POEM comparison on the following networks : canonical language (Baldassarre, Metcalf, Shulman, \& Corbetta, 2019), action language, action observation, motor execution and mental imagery networks (Courson \& Tremblay, 2018).

\section{Results}

All 10 participants benefited from POEM; improvements were observed with both trained and untrained items. In either case explored with fMRI, the recovery on verb naming following POEM was signed by distinct activation patterns. Specifically in P1, there was a significant activation in the left and right middle temporal gyri, the right fusiform and the left cerebellum, all of which are part of the canonical language network (Baldassarre et al., 2019). In P2, there was a significant activation in the right premotor cortex and the right cerebellum, which are both among the components of the sensorimotor network. Concurrently with behavioral recovery, a reduction in the number of recruited areas was observe in both participants.

\section{Discussion}

The evidence suggests that structured anomia treatment integrating sensorimotor strategies can improve word retrieval of treated and untreated verbs. In particular, POEM is the first verb anomia therapy protocol to show generalization of therapy effects to untreated items, and so across all participants. This finding suggests that POEM favors the implementation of a strategy that can be generalized to untrained items. The post-intervention activation pattern observed in the two participants, suggests that the sensorimotor character of POEM favors the recruitment of preserved elements of two brain networks, and so in relation to lesion size and location. In P1, a component of the sensorimotor circuit- the right premotor cortex, and the cerebellum, and in P2 the cerebellum and middle temporal gyri, supporting the mental representation of verb meaning, as well as the syntactic templates of nouns and verbs (Hagoort, 2016). Furthermore, the middle temporal gyrus is a component of two language processing relevant networks, namely the action language network and action observation network (Courson \& Tremblay, 2018), and the language canonical network Baldassarre et al., 2019). Preliminary analyses of functional connectivity data indicates that POEM favors the interactivity between these two networks supporting the recovery from verb anomia. Finally, POEM leads to more efficient use of brain resources, with less scattered activations and better synchronization of 
large-scale relevant networks, to sustain recovery by the implementation of a strategy that can be generalized to untreated items.

\section{References}

Baldassarre, A., Metcalf, N. V., Shulman, G. L., \& Corbetta, M. (2019). Brain networks' functional connectivity separates aphasic deficits in stroke. Neurology, 92(2), e125-e135.

Berroir, P., Ghazi-Saidi, L., Dash, T., Adrover-Roig, D., Benali, H., \& Ansaldo, A. I. (2017). Interference control at the response level: Functional networks reveal higher efficiency in the bilingual brain. Journal of Neurolinguistics, 43, 4-16.

Conroy, P., Sage, K., \& Lambon Ralph, M. A. (2006). Towards theory-driven therapies for aphasic verb impairments: A review of current theory and practice. Aphasiology, 20(12), 1159-1185. doi:10.1080/02687030600792009

Courson, M., \& Tremblay, P. (2018). The neural network of action language: a comparative meta-analysis. Paper presented at the Society for the Neurobiology of Language, Quebec, Canada.

Hagoort, P. (2016). Chapter 28 - MUC (Memory, Unification, Control): A Model on the Neurobiology of Language Beyond Single Word Processing. In G. Hickok \& S. L. Small (Eds.), Neurobiology of Language (pp. 339-347). San Diego: Academic Press.

Rofes, A., Capasso, R., \& Miceli, G. (2015). Verb production tasks in the measurement of communicative abilities in aphasia. Journal of Clinical and Experimental Neuropsychology, 37(5), 483-502. doi:10.1080/13803395.2015.1025709

Webster, J., \& Whitworth, A. (2012). Treating verbs in aphasia: exploring the impact of therapy at the single word and sentence levels. International Journal of Language and Communication Disorders, doi:10.1111/j.1460-6984.2012.00174.x 


\title{
Lexical Frequency and Lexical Semantics interact with Cognitive Reserve
}

\author{
Sonia Montemurro ${ }^{1,2}$, Gonia Jarema ${ }^{3}$, Sara Mondini ${ }^{1,2}$ \\ ${ }^{1}$ Department of General Psychology, University of Padua, Padua, Italy \\ ${ }^{2}$ Human Inspired Technology Research-Centre, University of Padua, Padua Italy \\ ${ }^{3}$ Université de Montréal and Research Centre, Institut universitaire de gériatrie de Montréal, \\ Montréal, Canada
}

\section{Introduction}

Cognitive Reserve derives from the level of education, working activity and leisure activity of an individual. The mechanism of acquiring new learnings is able to create, supply and repair neural resources in support of age-related neurological decline (Cabeza et al., 2018) through the modulation of cognitive performance (Stern et al., 1992; Darby et al., 2017, Thow et al., 2018). Neural networks underlying Cognitive Reserve can be significantly activated as cognitive demand increases (e.g., Cabeza et al., 2018; Darby et al., 2017), indicating that within the same task, the individual behavioral outcome is sensitive to both the task demand and the level of Cognitive Reserve of who is performing the task. In the language domain, the role of Cognitive Reserve has been explored to some extent, showing better language performance with high Cognitive Reserve over adulthood (e.g., Thow et al., 2018). However, most of the studies have measured language performance without considering the possible connection between Cognitive Reserve and psycholinguistic features (Hultsch et al., 1993; Thow et al., 2018). In our study, indeed, we observed the role of Cognitive Reserve across different variables such as Lexical Frequency (low vs. high) and Lexical Semantics (abstract vs. concrete). Since the involvement of Cognitive Reserve should be higher with the increase of task demand, we hypothesized a stronger effect of Cognitive Reserve in the case of low frequency words and abstract words, rather than with high frequency words and concrete words.

\section{Methods}

\section{Participants and Materials}

Sixty-five participants aged from 59 to 87 years underwent the Montreal Cognitive Assessment (MoCA, Nasreddine et al., 2005) to measure their cognitive profile and the Cognitive Reserve Index questionnaire (CRIq, Nucci et al., 2012) to measure their Cognitive Reserve Index (CRI). They performed a simple Lexical Decision task and a Semantic Matching task to evaluate lexical richness.

\section{Procedure}

Participants were administered the MoCA and the CRIq. The MoCA test is a neuropsychological screening assessing the global cognitive profile. The raw scores were adjusted for age and education according to the Italian norms (Conti et al., 2015). The CRIq is a semi-structured interview aimed to obtain a standardized quantitative measure of activities carried on during the lifespan, where the CRI score is an estimation of the CR. The CRI-total comprises three sub-components: education (CRI-Education), working activities (CRI-WorkingActivity), and leisure activities (CRI-LeisureTime). These scores are adjusted for age via a regression-based method to allow comparisons between groups of different ages (see Nucci et al., 2012). The Lexical Decision task included real words and non-words. In the Semantic Matching task, participants responded to synonyms and unrelated words with 'yes' or 'no' to indicate whether the paired words had the same meaning, or not. 


\section{Results}

The analyses were carried out using Generalized Linear Mixed-effect Models (Bates, Mächler, Bolker, \& Walker, 2014; Baayen et al., 2008; Quené and Van den Bergh, 2008). Our dependent variables were Response Times (RT, in milliseconds) and Accuracy (as binomial responses). Our predictors of interest were the cognitive reserve (CRI score), Lexical Frequency (i.e., high vs. low) and Lexical semantic (i.e., concrete vs. abstract). - In the Lexical Decision task, model comparison made by Akaike Information Criterion (AIC, Sakamoto et al., 1986) indicated that higher CRI-Education was the best predictor of RT, and the model fit was better when considering Lexical semantics in interaction with cognitive reserve. CRI-total predicted Lexical Decision Accuracy and, in line with our hypothesis, the effect of Cognitive Reserve was stronger for abstract words than for concrete words (Fig. 1a). - In the Semantic Matching task, participants with higher CRI-total showed significantly faster RT compared to participants with lower Cognitive Reserve. Performance was significantly more accurate in older adults with higher Cognitive Reserve, with stronger effects for low frequency words compared to high frequency words (Fig. 1b).
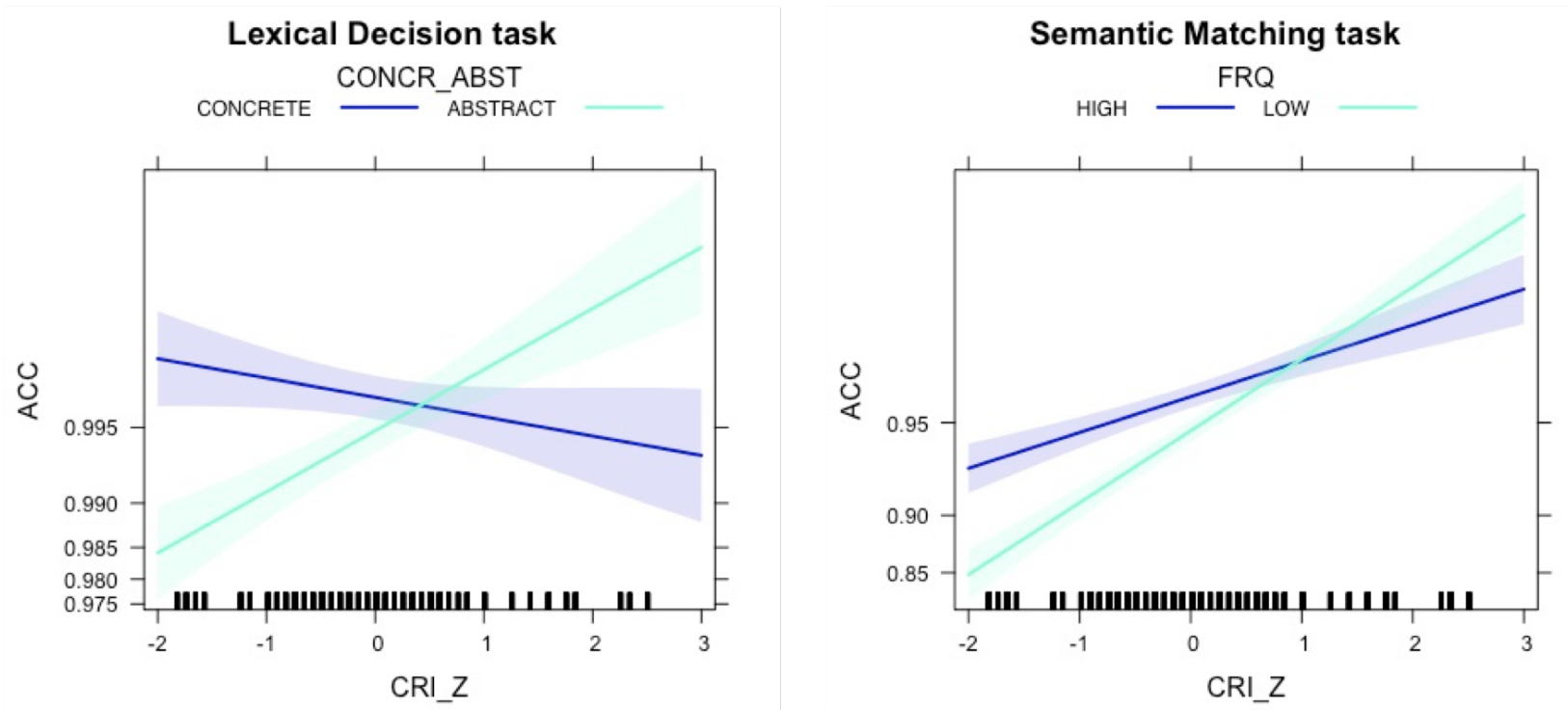

Fig. 1: a) On the left-hand side, it is shown the interaction effect between Cognitive Reserve and Lexical semantics in the Lexical Decision task; b) on the right-hand side, it is shown the interaction effect between Cognitive Reserve and Lexical Frequency in the Semantic Matching task. ACC = Accuracy; CONCR_ABST $=$ Lexical semantic; FRQ = lexical frequency.

\section{Discussion}

Both the Lexical Decision task and the Semantic Matching task were performed significantly better by participants with higher Cognitive Reserve in terms of RT and Accuracy. Cognitive Reserve predicted better processing of low frequency words and abstract words. These results indicate that better language performance can be predicted by higher Cognitive Reserve: a benefit that appears to be marked when more "difficult" stimuli (i.e. low frequency and abstract words) are being processed.

\section{References}

Baayen, R. H., Davidson, D. J., \& Bates, D. M. (2008). Mixed-effects modeling with crossed random effects for subjects and items. Journal of memory and language, 59(4), 390-412.

Bates, D., Mächler, M., Bolker, B., \& Walker, S. (2014). Fitting linear mixed-effects models using lme4. arXiv preprint arXiv:1406.5823. 
Conti, S., Bonazzi, S., Laiacona, M., Masina, M., \& Coralli, M. V. (2015). Montreal Cognitive Assessment (MoCA)-Italian version: regression based norms and equivalent scores. Neurological Sciences, 36(2), 209-214.

Darby, R. R., Brickhouse, M., Wolk, D. A., \& Dickerson, B. C. (2017). Effects of cognitive reserve depend on executive and semantic demands of the task. J Neurol Neurosurg Psychiatry, jnnp-2017

Cabeza, R., Albert, M., Belleville, S., Craik, F. I., Duarte, A., Grady, C. L., ... \& Rugg, M. D. (2018). Maintenance, reserve and compensation: the cognitive neuroscience of healthy ageing. Nature Reviews Neuroscience, 1.

Hultsch, D. F., Hammer, M., Small, B. J. (1993). Age differences in cognitive performance in later life: Relationships to self-reported health and activity life style. Journal of gerontology, 48(1), P1-P11. doi 10.1093/geronj/48.1.P1

Nasreddine, Z. S., Phillips, N. A., Bédirian, V., Charbonneau, S., Whitehead, V., Collin, I. et al. (2005). The Montreal Cognitive Assessment, MoCA: a brief screening tool for mild cognitive impairment. Journal of the American Geriatrics Society, 53(4), 695-699. doi: 10.1111/j.1532-5415.2005.53221.x

Nucci, M., Mapelli, D., Mondini, S. (2012). Cognitive Reserve Index questionnaire (CRIq): a new instrument for measuring cognitive reserve. Aging clinical and experimental research, $24(3), 218-226$.

Quené, H., \& Van den Bergh, H. (2008). Examples of mixed-effects modeling with crossed random effects and with binomial data. Journal of Memory and Language, 59(4), 413-425.

Sakamoto, Y., Ishiguro, M., \& Kitagawa, G. (1986). Akaike information criterion statistics. Dordrecht, The Netherlands: D. Reidel, 83, 902-926.

Stern, Y., Alexander, G. E., Prohovnik, I., \& Mayeux, R. (1992). Inverse relationship between education and parietotemporal perfusion deficit in Alzheimer's disease. Annals of Neurology: Official Journal of the American Neurological Association and the Child Neurology Society, 32(3), 371-375.

Thow, M. E., Summers, M. J., Saunders, N. L., Summers, J. J., Ritchie, K., \& Vickers, J. C. (2018). Further education improves cognitive reserve and triggers improvement in selective cognitive functions in older adults: The Tasmanian Healthy Brain Project. Alzheimer's $\&$ Dementia: Diagnosis, Assessment $\&$ Disease Monitoring, 10, 22-30. 
Poster Session I 


\title{
Inflecting regular and irregular verbs: neuroimaging and behavioural data from the three Italian conjugations
}

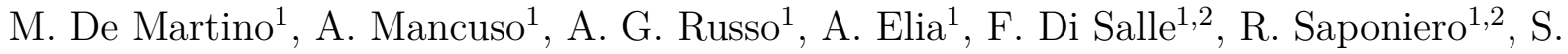 \\ Vietri $^{1}$, F. Esposito ${ }^{1,2}$, A. Laudanna ${ }^{1}$ \\ ${ }^{1}$ University of Salerno, Fisciano (Salerno), Italy \\ ${ }^{2}$ University Hospital "San Giovanni di Dio e Ruggi D'Aragona", Salerno, Italy
}

\section{Introduction}

The present study investigates the production of inflected forms from word-stems with a regular vs. an irregular inflection. This issue has been mostly focused on the theoretical debate between Dual Mechanism theories (rule-based procedures vs. recovery of whole forms stored in an associative network, Pinker \& Ullman, 2002) and Connectionist models (single procedure for both regular and irregular forms, McClelland \& Patterson, 2002). The two accounts make different predictions about the neural underpinnings of lexical processing for regular and irregular verbs. The declarative/procedural model is a modular theory positing that irregular forms are recovered from the mental lexicon which depends on declarative memory and is subserved by the temporal lobe, whereas regular forms are assembled by means of a mental grammar which involves procedural memory and is subserved by the frontal cortex and the basal ganglia (Ullman, 2001). Concurrent models do not postulate a categorical difference between regular and irregular forms and suggest that different pattern of brain activations elicited by regular and irregular verbs are predicted by phonological, semantic and other probabilistic features of verbs (Joanisse \& Seidenberg, 2005).

However, an alternative hypothesis, mainly grounded on linguistic, behavioral and simulation data (Burzio, 1998; Bordag \& Pechmann, 2009; Colombo et al., 2004; 2006; Laudanna, 2007; Laudanna et al., 2004; Pirrelli \& Herreros, 2005; Verissimo \& Clahsen, 2009), suggests that all inflected forms in the mental lexicon, regardless of regularity, are organized in clusters on the basis of their paradigmatic relations among word-forms other than the regular/irregular distinction and that inflectional processes are likely to be governed by membership of words to specific clusters, namely the inflectional classes. This third positions predicts the possibility to detect experimental effects driven by the membership of words to inflectional classes rather than to the coarse distinction between regular and irregular.

In this study we try to shed light on the issue by exploiting a suitable test case, the Italian verb inflectional system. Here, verbs fall into one of three conjugations each exhibiting different degrees of regularity, numerosity and consistency.

By using both behavioral and neuroimaging techniques, we aim at demonstrating that mechanisms underlying speaker's ability to perform inflectional operations rely on grammatical properties of verbs depending on their membership to different inflectional classes.

\section{Methods}

A past-participle generation task was admnistered in a behavioral (42 participants) and an event-related fMRI setting (23 participants). A set of 168 Italian verbs was selected and arranged into 6 subsets of experimental stimuli on the basis of Regularity (regulars vs. irregulars) and Conjugation membership (1st vs. 2nd vs. 3rd). The six subsets were matched on frequency, length, N-count, locus and manner of articulation of the initial phoneme, syllabic structure of the initial syllable, syllabic structure of both the infinitive and past-participle form.

Different sets of statistical analyses were carried out on response latencies, accuracy rates and BOLD responses. Analyses testing putative effects of Conjugation were restricted to response patterns only elicited by regular verbs. Analyses on response measures only elicited by 2nd and 3rd conjugation verbs tested the interaction between Regularity and Conjugation. 


\section{Results}

Main effects of the variable Conjugation were obtained both in the behavioural and in the fMRI experiments: 2nd conjugation regular verbs were responded significantly slower and poorer than 1 st and 3rd conjugation regular verbs. The left middle frontal gyrus and the pre-supplementary motor area exhibited a significantly higher BOLD activation response for 2 nd and 3rd conjugation regular verbs than for 1st conjugation regular verbs.

In addition, the analyses performed on both 2nd and 3rd conjugation regular and irregular verbs revealed that 2 nd conjugation verbs were slower and less accurate than 3rd conjugation verbs and that the perigenual part of the left anterior cingulate cortex was significantly more activated for 2 nd than for 3rd conjugation verbs. A main effect of Regularity was also observed: irregular verbs were slower and less accurate than regular verbs. The left prefrontal cortex and the anterior part of the left inferior frontal gyrus were found significantly more activated for irregular when compared to regular verbs.

Interestingly, a significant Conjugation by Regularity interaction was observed: behaviorally, the Regularity effect was stronger for 3rd than for 2nd conjugation verbs. In the left and right post-central gyri, the 2nd conjugation irregular verbs elicited significant weaker activation than regular ones; the 3rd conjugation verbs elicited the opposite pattern.

\section{Discussion}

The present study demonstrates that inflecting Italian verbs from different conjugations, irrespective of regularity, requires different cognitive operations since it elicits differences in behavioral response latencies, accuracy rates and cortical activations. Rather than a segregation between different brain structures devoted to the processing of regular and irregular verbs, different patterns of activation for both the regular and irregular verbs in the same structures were found. Differences among regular verbs belonging to different inflectional classes were also obtained both on behavioral and neural grounds.

This pattern of data cannot be easily accommodated within either a dual or a single mechanism account. Dual mechanism theories postulate that all regular verbs follow symbolic inflectional rules, thereby no differences among regular verbs are expected. The observed difference cannot be ascribed neither to any formal feature as predicted by single mechanism accounts: actually, in our work we matched many formal features across the experimental categories.

Our data corroborate the idea that language-specific properties of inflectional classes might modulate cognitive operations and biological underpinnings of inflectional behavior.

\section{References}

Bordag, D., \& Pechmann, T. (2009). Externality, internality, and (in) dispensability of grammatical features in speech production: Evidence from Czech declension and conjugation. Journal of Experimental Psychology: Learning, Memory, and Cognition, 35(2), 446.

Burzio, L. (1998). Multiple correspondence. Lingua, 104(1-2), 79-109.

Colombo, L., Laudanna, A., De Martino, M., \& Brivio, C. (2004). Regularity and/or consistency in the production of the past-participle? Brain and Language, 90(1), 128-142.

Colombo, L., Stoianov, I., Pasini, M., \& Zorzi, M. (2006). The role of phonology in the inflection of Italian verbs: A connectionist investigation. The Mental Lexicon, 1(1), 147181.

Joanisse, M. F., \& Seidenberg, M. S. (2005). Imaging the past: Neural activation in frontal and temporal regions during regular and irregular past-tense processing. Cognitive, Affective, E) Behavioral Neuroscience, 5(3), 282-296.

Laudanna, A. (2007). Representation and processing of regular and sub-regular verbal forms in Italian. Lingue e Linguaggio, 6(2), 227-246. 


\begin{tabular}{|c|c|c|c|c|c|c|}
\hline & \multicolumn{2}{|c|}{$\begin{array}{l}\text { Main effect of } \\
\text { Conjugation }\end{array}$} & & & & \\
\hline & reaction times & $\begin{array}{l}\text { error } \\
\text { rate }\end{array}$ & & & & \\
\hline $1^{\text {st }}$ conjugation verbs & $548 \mathrm{~ms}(42)$ & $6 \%$ & & & & \\
\hline $2^{\text {nd }}$ conjugation verbs & $575 \mathrm{~ms}(48)$ & $16 \%$ & & & & \\
\hline \multirow[t]{4}{*}{$3^{\text {rd }}$ conjugation verbs } & $558 \mathrm{~ms}(46)$ & $13 \%$ & & & & \\
\hline & \multicolumn{4}{|c|}{$\begin{array}{c}\text { Regularity by Conjugation } \\
\text { Interaction }\end{array}$} & & \\
\hline & \multicolumn{2}{|c|}{ regular verbs } & \multicolumn{2}{|c|}{ irregular verbs } & & \\
\hline & reaction times & $\begin{array}{l}\text { error } \\
\text { rate }\end{array}$ & reaction times & \begin{tabular}{|l} 
error \\
rate
\end{tabular} & reaction times & error rate \\
\hline $2^{\text {nd }}$ conjugation & $577 \mathrm{~ms}(47)$ & $20 \%$ & $577 \mathrm{~ms}(46)$ & $30 \%$ & 577 ms (46) & $25 \%$ \\
\hline \multirow[t]{2}{*}{$3^{\text {rd }}$ conjugation } & $558 \mathrm{~ms}(46)$ & $13 \%$ & $571 \mathrm{~ms}(49)$ & $27 \%$ & $564 \mathrm{~ms}(48)$ & $20 \%$ \\
\hline & $567 \mathrm{~ms}(48)$ & $16 \%$ & 574 ms (47) & $28 \%$ & & \\
\hline Region & \multicolumn{2}{|c|}{ Talairach } & \multicolumn{2}{|l|}{ Cluster size } & \multicolumn{2}{|c|}{ P-value } \\
\hline \multicolumn{7}{|c|}{ Model: 1-ANOVA (Conjugation) } \\
\hline L-MFG & \multicolumn{2}{|c|}{$-45,-7,+41$} & 521 & & \multicolumn{2}{|c|}{0.000320} \\
\hline L-PreSMA & \multicolumn{2}{|c|}{$-3,+5,+49$} & 405 & & \multicolumn{2}{|c|}{0.000445} \\
\hline L-Caudate & \multicolumn{2}{|l|}{$-8,+8,+4$} & $231^{*}$ & & \multicolumn{2}{|c|}{0.000904} \\
\hline R-Caudate & \multicolumn{2}{|c|}{$+6,+2,+4$} & $55^{*}$ & & \multicolumn{2}{|c|}{0.000532} \\
\hline \multicolumn{7}{|c|}{ Model: 2-ANOVA (Regularity) } \\
\hline L-PFC & \multicolumn{2}{|c|}{$-48,-1,+25$} & 2663 & & \multicolumn{2}{|c|}{0.000268} \\
\hline L-IFG & \multicolumn{2}{|c|}{$-45,+29,+13$} & 1249 & & \multicolumn{2}{|c|}{0.000552} \\
\hline L-PreSMA & \multicolumn{2}{|c|}{$-6,-1,+49$} & $143^{*}$ & & \multicolumn{2}{|c|}{0.001038} \\
\hline L-Caudate & \multicolumn{2}{|c|}{$-12,+8,+7$} & $116^{*}$ & & \multicolumn{2}{|c|}{0.000303} \\
\hline L-Hipp. & \multicolumn{2}{|c|}{$-27,-34,-2$} & $201^{*}$ & & \multicolumn{2}{|c|}{0.004747} \\
\hline \multicolumn{7}{|c|}{ Model: 2-ANOVA (Conjugation) } \\
\hline L-ACC & \multicolumn{2}{|c|}{$-6,+29,+1$} & 388 & & \multicolumn{2}{|c|}{0.000753} \\
\hline
\end{tabular}

Table 1: Mean reaction times (standard deviations in parentheses), error rates and clusters of significant fMRI changes. 
Laudanna, A., Gazzellini, S., \& De Martino, M. (2004). Representation of grammatical properties of Italian verbs in the mental lexicon. Brain and Language, 90(1-3), 95-105.

McClelland, J. L., \& Patterson, K. (2002). Rules or connections in past-tense inflections: What does the evidence rule out? Trends in Cognitive Sciences, 6(11), 465-472.

Pinker, S., \& Ullman, M. T. (2002). The past and future of the past tense. Trends in Cognitive Sciences, 6(11), 456-463.

Pirrelli, V., \& Herreros, I. (2005). Learning morphology by itself. Paper presented at the 5th Mediterranean Morphology Meetings, Bologna.

Ullman, M. T. (2001). A neurocognitive perspective on language: The declarative/procedural model. Nature Reviews Neuroscience, 2(10), 717-7.

Veríssimo, J., \& Clahsen, H. (2009). Morphological priming by itself: A study of Portuguese conjugations. Cognition, 112(1), 187-194. 


\title{
Confrontation-naming and connected speech production in early post-stroke aphasia
}

\author{
Johémie Boucher ${ }^{1,2}$, Karine Marcotte ${ }^{3,4}$, Amélie Brisebois ${ }^{3}$, Marianne Désilets-Barnabé ${ }^{3,4}$, \\ Alberto Osa García ${ }^{3,4}$, Elizabeth Rochon ${ }^{5,6,7,8}$, Carol Leonard ${ }^{9}$, Alex Desautels ${ }^{3,10}$, Simona M. \\ Brambati ${ }^{1,2}$ \\ ${ }^{1}$ Centre de recherche de l'Institut Universitaire de Gériatrie de Montréal, Montréal, Québec, \\ Canada \\ ${ }^{2}$ Département de psychologie, Faculté des arts et des sciences, Université de Montréal, \\ Montréal, Québec, Canada \\ ${ }^{3}$ Centre de recherche du Centre intégré universitaire de santé et de services sociaux du \\ Nord-de-l'T̂le-de-Montréal, Montréal, Québec, Canada \\ ${ }^{4}$ École d'orthophonie et d'audiologie, Faculté de médecine, Université de Montréal, Montréal, \\ Québec, Canada \\ ${ }^{5}$ Department of Speech-Language Pathology, Faculty of Medicine, University of Toronto, \\ Toronto, Canada \\ ${ }^{6}$ Toronto Rehabilitation Institute, Toronto, Canada \\ ${ }^{7}$ Heart and Stroke Foundation, Canadian Partnership for Stroke Recovery, Ontario, Canada \\ ${ }^{8}$ Rehabilitation Sciences Institute, University of Toronto \\ ${ }^{9}$ School of Rehabilitation Sciences, University of Ottawa \\ ${ }^{10}$ Département des neurosciences, Faculté de médecine, Université de Montréal, Montréal, \\ Québec, Canada.
}

\section{Introduction}

Post-stroke aphasia (PSA) is an acquired impairment of speech and language caused by a stroke, generally in the left hemisphere. The impacts of PSA on language production are usually objectivized using tasks relying on single-word production (e.g., confrontation-naming tasks), which generally reveal that all people with aphasia experience some degree of naming impairment (Goodglass \& Wingfield, 1997).

Research has consistently shown that PSA affects word production not only in isolation, but also in connected speech production (Kavé \& Goral, 2017). Accordingly, recent literature highlights the importance of the analysis of connected speech production among patients with PSA (Mayer \& Murray, 2003), which could provide a more detailed and ecological examination of language production impairments.

In clinical settings, connected speech samples are often elicited by picture description tasks. This procedure, as opposed to other speech-elicitation methods (e.g., structured or semistructured interviews), elicits relatively constrained speech, thus facilitating speech comparison between groups (Chenery \& Murdoch, 1994). Connected speech analysis showed that PSA can affect multiple speech and language domains, such as speech production, semantic content, and morphosyntactic and lexical measures (Fergadiotis \& Wright, 2016; Kavé \& Goral, 2017).

Studies quantitatively documenting the effects of PSA on connected speech elicited by picture description tasks, especially in the early stages of the disease, are rather scarce and have yielded mixed results. Moreover, the relationship between the performance on naming tests (i.e. singleword production) typically used in clinical evaluations and the performance on speech-eliciting procedures remains unclear. While some findings suggest that confrontation-naming measures in chronic PSA patients are associated with measures of word-retrieval in connected speech production (Fergadiotis \& Wright, 2016; Herbert, Best, Hickin, Howard, \& Osborne, 2003), 
others support a dissociation between the performance of people with aphasia in single-word and discursive tasks (Crutch \& K Warrington, 2004; Mayer \& Murray, 2003).

Thus, our objectives will be:

a. to assess the effects of PSA on quantitative measures of connected speech production in the subacute stage (10-14 days post-stroke)

b. to determine the relationship between the performance in confrontation naming and connected speech measures among the same people with PSA

\section{Methods}

Participants

Twenty participants with PSA (age: $M=71.85$; male (female): 13(7)) and 21 healthy controls (age: $M=63.62$; female (male): 10(11)) were recruited in the stroke unit of Hôpital du SacréCoeur de Montréal. All participants were right-handed and participants in the PSA group had suffered from a single ischemic middle cerebral artery stroke in the left hemisphere. Participants in the PSA group were evaluated between ten and fourteen days post-stroke $(M=10.39)$.

\section{Language assessment}

For all participants, connected speech elicited by the Picnic scene (Western Aphasia Battery) was recorded. The speech samples were then transcribed by a trained speech-language pathologist using the CLAN program (MacWhinney, 2000). Measures of speech quanta (duration, total number of words, types, open class words, and closed class words), speech rate (words per minute), errors (repetition, self-correction, and word error rates), lexical diversity (type-token ratio, VOC-D measure), lexical selection (open-to-closed class ratio and noun-to-verb ratio), informativity (information content units; information units for the healthy control group were manually extracted from connected speech samples of an other control group sharing similar sociodemographic data), efficiency/density (information units/duration, information units/total number of words, and density), and mean length of utterance were semi-automatically extracted. All participants were also administered a standardized test assessing confrontationnaming (DO80).

\section{Statistical analysis}

We contrasted the performance of PSA participants and healthy controls on the confrontationnaming score and the connected speech measures using independent samples t-tests. Then, for the connected speech measures for which we found significant differences between the PSA and the healthy control group, we tested for correlations with the confrontation-naming task within the PSA group only. Finally, we conducted additional linear regression analyses to identify the strongest connected speech predictors of performance on the confrontation-naming task.

\section{Results}

Results of group comparison between the PSA group and healthy controls for confrontationnaming measures and connected speech production are presented in Table 1.

\section{Discussion}

Our findings show that early PSA is associated with changes in multiple aspects of connected speech production, such as speech quanta measures, speech rate, informativity, lexical diversity, and mean length of utterance. While reduced lexical diversity, number of types, speech rate, and mean length of utterance were all good predictors of naming deficits, we found poor informativity of speech to be the best predictor of naming impairments on a standardized confrontation-naming task. 
Table 1. Performance of People with Aphasia and Healthy Controls on Confrontation-Naming and Speech Production Measures

\begin{tabular}{|c|c|c|c|c|c|c|}
\hline & \multicolumn{2}{|c|}{ PSA } & \multicolumn{2}{|r|}{$\mathrm{HC}$} & \multirow[t]{2}{*}{$t$} & \multirow[t]{2}{*}{$p$} \\
\hline & $\mathrm{M}$ & $\mathrm{SD}$ & $\mathrm{M}$ & $\mathrm{SD}$ & & \\
\hline DO80 (z-score) & -9.26 & 11.63 & 0.64 & 0.47 & -3.91 & $* *$ \\
\hline Duration (seconds) & 57.43 & 41.19 & 78.57 & 48.22 & -1.53 & \\
\hline TNW & 123.52 & 111.70 & 210.24 & 118.49 & -2.44 & $* *$ \\
\hline Types & 50.10 & 36.43 & 93.57 & 33.18 & -4.04 & $* *$ \\
\hline \#OCW & 36.43 & 34.61 & 69.29 & 37.75 & -2.94 & $* *$ \\
\hline \#CCW & 85.67 & 78.72 & 140.14 & 80.95 & -2.21 & $* *$ \\
\hline WPM & 110.41 & 48.86 & 171.92 & 38.47 & -4.53 & $* *$ \\
\hline Repetition rate (\%) & 3.05 & 3.04 & 0.75 & 0.72 & 3.38 & $* *$ \\
\hline Self-correction rate (\%) & 1.51 & 1.41 & 1.04 & 0.88 & 1.27 & \\
\hline Word error rate $(\%)$ & 5.20 & 7.72 & 0.06 & 0.18 & 3.06 & $* *$ \\
\hline TTR & 0.49 & 0.20 & 0.48 & 0.09 & 0.17 & \\
\hline VOC-D & 31.70 & 10.17 & 45.45 & 11.28 & -3.75 & $* *$ \\
\hline OCW-CCW ratio & 0.42 & 0.20 & 0.51 & 0.20 & -1.91 & \\
\hline Noun-to-verb ratio & 7.27 & 7.47 & 6.97 & 3.48 & 0.16 & \\
\hline ICUs & 13.00 & 9.45 & 24.22 & 4.23 & -5.26 & $* *$ \\
\hline ICUs/duration & 0.14 & 0.07 & 0.13 & 0.05 & 0.66 & \\
\hline $\mathrm{ICUs} / \mathrm{tnw}$ & 0.27 & 0.16 & 0.29 & 0.13 & -0.38 & \\
\hline Density & 0.25 & 0.13 & 0.34 & 0.06 & -2.89 & $* *$ \\
\hline MLU & 6.03 & 3.96 & 10.39 & 2.40 & -4.32 & $* *$ \\
\hline
\end{tabular}

${ }^{* *} p<0.01 ; \mathrm{PSA}=$ post-stroke aphasia group; $\mathrm{HC}=$ healthy controls; TNW = total number of words; \#OCW $=$ number of open-class words; $\# \mathrm{CCW}=$ number of closed-class words; WPM $=$ words per minute; $\mathrm{TTR}=$ type-token ratio; OCW-CCW ratio = open-to-closed-class-word ratio; ICUs = number of information content units conveyed; ICUs/words = number of information content units/total number of words produced; ICUs/duration = number of information content units/duration in seconds; MLU = mean length of utterance.

Picture description tasks are quick, easy to administer, and provide a detailed approximation of language production abilities in subacute PSA. While recent recommendations highlight the importance of conducting complete language evaluations in the first days following stroke, this procedure could prove useful for rapid assessment in early PSA. Future directions for this project could include measuring the relationship between quantitative measures of connected speech production in PSA and the performance on standardized single-item tasks targeting other language functions, such as semantic and phonological processing.

\section{References}

Chenery, H. J., \& Murdoch, B. E. (1994). The production of narrative discourse in response to animations in persons with dementia of the Alzheimer's type: Preliminary findings. Aphasiology, 8(2), 159-171. doi:10.1080/02687039408248648

Crutch, S., \& K Warrington, E. (2004). Preservation of Propositional Speech in a Pure Anomic: The Importance of an Abstract Vocabulary (Vol. 9).

Fergadiotis, G., \& Wright, H. H. (2016). Modelling confrontation naming and discourse performance in aphasia. Aphasiology, 30(4), 364-380.

Goodglass, H., \& Wingfield, A. (1997). Anomia: Neuroanatomical and cognitive correlates: Academic Press.

Herbert, R., Best, W., Hickin, J., Howard, D., \& Osborne, F. (2003). Combining lexical and interactional approaches to therapy for word finding deficits in aphasia. Aphasiology, $17(12), 1163-1186$.

Kavé, G., \& Goral, M. (2017). Do age-related word retrieval difficulties appear (or disappear) in connected speech? Aging, Neuropsychology, and Cognition, 24(5), 508-527. doi:10.1080/13825585.2016.1226249 
MacWhinney, B. (2000). The CHILDES Project: Tools for Analyzing Talk. 3rd Edition. Mahwah, NJ: Lawrence Erlbaum Associates

Mayer, J., \& Murray, L. (2003). Functional measures of naming in aphasia: Word retrieval in confrontation naming versus connected speech. Aphasiology, 17(5), 481-497. 


\title{
Investigating the potential of structural priming as a form of constraint-induced language therapy in post-stroke aphasia
}

\author{
Martina Garzon ${ }^{1}$, Federica Biddau ${ }^{1}$, Giorgio Arcara ${ }^{1}$, Francesca Meneghello ${ }^{1}$, Daniela \\ D'Imperio $^{1}$, Giulia Bencini ${ }^{2}$ \\ ${ }^{1}$ San Camillo Hospital, Venezia \\ ${ }^{2}$ Università Ca' Foscari Venezia
}

\section{Introduction}

Structural priming is the tendency to produce previously processed sentence structures. In the first experimental study on this topic (Bock, 1986), priming in English monolingual unimpaired speakers was found with Active/Passive and Ditransitive/Prepositional Dative sentence structures. Priming is useful to investigate the architecture and mechanisms of language processing (Pickering \& Ferreira 2008, Bencini 2013; 2017; Branigan and Pickering, 2016). By orthogonally manipulating the overlapping dimensions between prime and target (e.g., structure, semantics, etc.) priming allows to isolate the dimensions to which the language processing system is sensitive to (e.g., Bock and Loebell 1992). Some studies used structural priming in patients with aphasia and results suggest that it increases the chance to produce complex sentences (Cho and Mack, 2010; Cho, Mack and Thompson, 2016).

The aims of the current study are:

- to examine passive production in Italian speakers with aphasia using structural priming,

- to assess if priming can be an effective language intervention in speakers with aphasia.

\section{Methods}

Participants

Data collection is currently in progress. Overall, we plan to collect data from 10 Italian speakers with aphasia hospitalized in a rehabilitation hospital. All participants have a stroke in the left hemisphere, no neurological and speech-language disorders prior to their stroke, right-handed and have no impairment visual and hearing acuity.

\section{Stimuli and tasks}

Assessment: we administer Aachener Aphasie Test, Italian version, Luzzatti et at., 1996; visual and oral sentences comprehension; oral description of pictures; oral controlled description of images; visual and oral grammatically judgments of the Batteria per l'Analisi dei Deficit Afasici (Miceli et at., 1994); the task 55 from Psycholinguistic assessments of language processing in aphasia (Kay et al., 1996); Assessment of Pragmatic Abilities and Cognitive Substrates (Arcara \&Bambini, 2016). In addition, we collect a narrative sample.

The experiment is administered via Psychopy software (Peirce, 2007). For the experiment we use a total of 32 prime sentences (16 actives and 16 passives) that are paired with 16 pictures of target repeated twice. All sentences have one inanimate agent (e.g. rock) and one animate patient (e.g. boy) and transitive verb (e.g. hit). Prime sentences are recorded. Target pictures are 16 black and white line drawings adapted from Bencini, Bock. The position of the agent is counterbalanced (half is on the right and half is on the left). And the infinitive form of the verb is written under the picture.

\section{Procedure}

Participants receive 3 hourly sessions of assessment and 2 hourly sessions for the experimental tasks, which took place over three weeks with a five days break between T1 and T2. 
TO

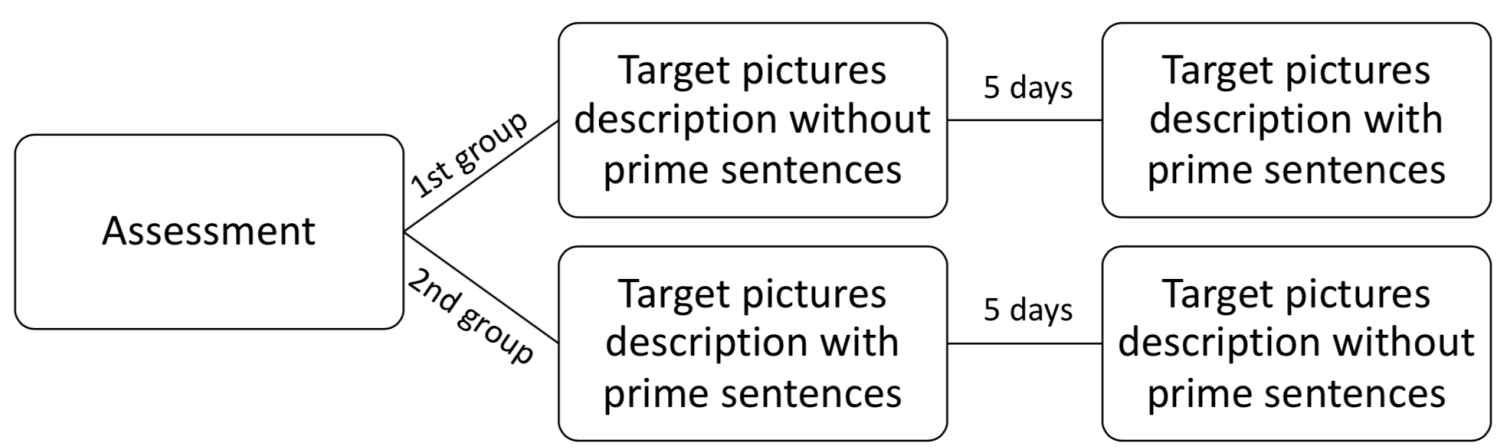

After the assessment, the patients are divided into two groups. Group one is going to describe target picture without prime sentences at T1. After five days participants describe the same pictures with prime sentences at T2. Group two receives the experimental tasks in reverse order.

At the beginning of the experimental task with structural priming the instructions are presented. Then there is a white slide with a written sentence. A recorded voice reads aloud the sentence and the participant has to repeat aloud the sentence that he heard. Then the patient has to fix a blank slide associated with a long sound. After that, the target picture comes up and the participant has to try to describe the following picture with the same structural form that he repeated before.

In the experimental task without structural priming, patients are required to describe the pictures without any structural form restrictions.

\section{Data analysis}

The outcomes to be measured are:

1. the accuracy in the production after the prime sentences (correct responses included active and passive responses produced in corresponding prime conditions)

2. the number of passive sentences produced in the experimental task without prime condition

In order to evaluate the role of the priming, a priming index (PI) is calculated on the ratio between the occurrences of the structural forms of the target descriptions matching with the prime and the total amount of descriptions. A mixed model will be employed to analyze the effect of the type of prime on the priming index. In addition, the without prime condition task will be used to assess possible long effects of the previous prime task. For instance, in a mixed model the percentage of passive sentences is analyzed on the effect of time (in T1 vs. T2).

\section{Results}

Data collection is currently in progress and preliminary results will be presented at the XX conference. 


\section{References}

Arcara, G., \& Bambini, V. (2016). A test for the assessment of pragmatic abilities and cognitive substrates (APACS): Normative data and psychometric properties. Frontiers in psychology, $7,70$.

Bencini G. (2013) Psycholinguistics in Hoffmann, T., \& Trousdale, G. (Eds.). (2013). The Oxford handbook of construction grammar. Oxford University Press.

Bencini, G. M. (2017). Speech Errors as a Window on Language and Thought: A Cognitive Science Perspective. Altre Modernità, 243-262.

Bock, J. K. (1986). Syntactic persistence in language production. Cognitive psychology, 18(3), 355-387.

Bock, K., Loebell, H., \& Morey, R. (1992). From conceptual roles to structural relations: Bridging the syntactic cleft. Psychological review, 99(1), 150.

Branigan H. and M. Pickering, 2016, "An Experimental Approach to Linguistic Representation", Behavioral and Brain Sciences, November, pp. 1-73.

Cho-Reyes, S., Mack, J. E., \& Thompson, C. K. (2016). Grammatical encoding and learning in agrammatic aphasia: Evidence from structural priming. Journal of memory and language, 91, 202-218.

Cho, S., \& Thompson, C. K. (2010). What goes wrong during passive sentence production in agrammatic aphasia: An eyetracking study. Aphasiology, $24(12), 1576-1592$.

Kay, J., Lesser, R., \& Coltheart, M. (1996). Psycholinguistic assessments of language processing in aphasia (PALPA): An introduction. Aphasiology, 10(2), 159-180.

Miceli, G., Laudanna, A., Burani, C., \& Capasso, R. (1994). Batteria per l'analisi dei deficit afasici. Cepsag, Roma.

Peirce, J. W. (2007). PsychoPy - Psychophysics software in Python. Journal of Neuroscience Methods, 162 (1-2):8-13

Pickering, M. J., \& Ferreira, V. S. (2008). Structural priming: a critical review. Psychological bulletin, $134(3), 427$. 


\title{
Adaptation of the Bilingual Aphasia Test to Lebanese Arabic
}

\author{
Nour Ezzeddine, Barbara Köpke \\ Octogone-Lordat, University of Toulouse
}

\section{Introduction}

Multiple languages coexist in Lebanon due to historical, cultural and educational circumstances (Bacha \& Bahous, 2011; Bahous \& al., 2014). Lebanese people are able to speak at least two languages from early childhood. They become bilingual speakers, using their languages on a daily basis and codeswitching frequently (ibid.). This linguistic diversity challenges the assessment of bilingual people with aphasia (BPWA) in Lebanon. The diagnosis of aphasia for bilinguals is based on the clinicians' professional knowledge about acquired language impairments and their use of assessment tools created for French or English-speaking countries. However, evaluation guidelines for BPWA highly recommend not only the examination of all the languages spoken by the patient but also documenting their history of bilingualism (Kiran \& Robert, 2012; Roberts, 2008). In order to improve the clinical practices of speech-language pathologists (SLP) and objectify the language assessment of BPWA, the short version of the Bilingual Aphasia Test (BAT, Paradis \& Libben, 1987) was adapted to Lebanese Arabic (LA), taking into consideration the specificities of the Lebanese dialect (Ezzeddine, 2017, 2018). The short version was chosen because of the necessity of assessing all languages and socio-economic factors (sessions not covered by insurance) interfering with the engagement of patients in therapy.

\section{Methods}

\section{Linguistic adaptation process}

The adaptation of the Lebanese BAT (L-BAT) includes 24 subtests for different oral skills and transcoding and 8 subtests involving literacy skills in Latinized Arabic. The adaptation and standardization of the L-BAT was conducted in two subsequent studies to guarantee better stimuli selection and allow further modifications if needed, especially for some subtests. For instance, in the auditory discrimination subtest, choice of stimuli is complex because of either lack of imaginability of some concepts, dialectal differences within Lebanon, and characteristics of Arabic languages: variability of pronunciation resides in the vowels while morphology is mainly consonantal (e.g. [ al] \& [ el] refer to the word "scarf"). Concerning the non-standard sentence type in the syntactic comprehension subtest, the passive construction, non-inexistent in Arabic dialects, was substituted with VSO forms since SVO forms are more common in oral communication (Khamis-Dakwar \& al., 2012; 2018).

\section{Data collection and analysis}

Twenty-eight healthy bilingual participants were recruited (75\% women; mean age=48.66 y.o.; mean education level=14.85 years). All participants were native speakers of LA, with French as a second language (L2); some of them also spoke English. One bilingual participant with aphasia was recruited in order to verify the applicability of the L-BAT with BPWA.

The patient, right handed female, 68 y.o. (71 at the time of the study), 14 years of schooling, had suffered a stroke in the left hemisphere. Her L1 is LA and L2 is French. The aphasia signs in the acute stroke indicated mutism followed by spontaneous recovery. The initial language assessment took place one-month post-stroke using non-standardized language tasks in LA. This revealed verbal production impairment characterized by anomia and phonological processing deficits, compensated with efficient phonological repair strategies and codeswitching to French. Language intervention had ended one year prior to inclusion. According to the patient, her language skills had improved; verbal deficits only increased with fatigue and stress. 
All participants completed the L-BAT and the French short version of the BAT (F-BAT). Data analysis aimed at 1) analyzing the success rate of the L-BAT, 2) comparing the performance of healthy participants on the Lebanese and French versions, 3) studying the correlation between BAT scores and sociodemographic variables (age and education level) and 4) exploring the results of the bilingual patient with aphasia.

\section{Results}

Results indicate a significantly better success rate for the L-BAT (92.98\%) than for the F-BAT $(92.07 \% ; \mathrm{Z}=-4,282 ; p<0.01)$ in healthy bilinguals. Analyses also demonstrate a correlation of L-BAT scores with age $(=-0,559 ; p<0.01)$ and education level $(=0.375 ; p<0.01)$ but not for F-BAT scores. Hence, performance in L1 Lebanese (but not L2 French) decreases with age and increases with higher education.

As for the patient, the BAT pinpointed detailed information about her history of bilingualism. As a native speaker of LA, acquired at home, her formal education was exclusively conducted in French. She reported being fluent in LA as much as in French, but she used to speak LA on a daily basis whereas French was spoken weekly only. However, she considered having better written language skills in French than Arabic and needed to use the latter less frequently than French. She also reported codeswitching before the stroke, which appeared in spontaneous speech and the naming task in French. Interestingly, L-BAT and F-BAT scores indicate relatively better performances in French (196/228) than in LA (207/291). More importantly, errors were spotted at specific subtests involving phonological processing such as repetition tasks, reading aloud tasks and spontaneous speech in both languages.

\section{Discussion}

The high success rates observed on the L-BAT validate our selection of stimuli despite the dialectal variation in Lebanon, specifically for the auditory discrimination task where scores reach $98.49 \%$. Furthermore, the effects of age and education level on the L-BAT are in line with other studies (Gómez-Ruiz \& al., 2012; Guilhem \& al., 2013). Conversely, the absence of this effect on the F-BAT could be explained by the heterogeneity in French proficiency among participants, regardless of their age and education level.

The patient's performances on the L-BAT and F-BAT support the phonological impairment established at her initial assessment, demonstrating the effectiveness of the L-BAT in aphasia diagnosis. Nevertheless, qualitative analysis of errors is a must for aphasia diagnosis because it supplements the clinical signs of aphasia and explains postmorbid language skills according to premorbid language habits (e.g., codeswitching).

Despite the work invested in the BAT adaptations to numerous languages, additional investigations are required to collect normative data on language assessment for bilinguals. Precisely, further studies about bilinguals in Arabic-speaking countries are crucially needed in order to improve language assessment and therapy for those individuals. For this purpose, the standardization of the Lebanese BAT is still ongoing.

\section{References}

Bacha, N. N., \& Bahous, R. (2011). Foreign Language Education in Lebanon: A Context of Cultural and Curricular Complexities. Journal of Language Teaching and Research, 2(6), $1320-1328$.

Bahous, R. N., Nabhani, M. B., \& Bacha, N. N. (2014). Code-switching in higher education in 
a multilingual environment: a Lebanese exploratory study. Language Awareness, 23(4), 353-368.

Ezzeddine, N. (2017). Adaptation du Bilingual Aphasia Test (Version Courte) au libanais : Données linguistiques et psychométriques. Mémoire de Master 1 en Sciences du Langage (LiCoCo), Toulouse, France. En ligne http://dante.univ-tlse2.fr/id/eprint/3788

Ezzeddine, N. (2018). Adaptation du Bilingual Aphasia Test au contexte linguistique libanais plurilingue : Étude du code-switching à l'épreuve de discours et analyse des fluences verbales. Mémoire de Master 2 en Sciences du Langage (LiCoCo), Toulouse, France. En ligne http://dante.univ-tlse2.fr/id/eprint/7003

Gómez-Ruiz, I., Aguilar-Alonso, Á., \& Gutiérrez-Cabello, L. (2012). Rendimiento de bilingües catalán-castellano en el test de Afasia para Bilingües de Michel Paradís: influencia de la edad y del nivel educativo. Anuario de Psicología, 42(3), 309-325.

Guilhem, V., Gomes, S., Prodhomme, K., \& Köpke, B. (2013). Le Screening BAT : un outil d'évaluation rapide disponible en 8 langues et adaptable à toutes les langues du BAT. Rééducation orthophonique, 253, 121-142.

Khamis-Dakwar, R., Froud, K., \& Gordon, P. (2012). Acquiring diglossia: mutual influences of formal and colloquial Arabic on children's grammaticality judgments. Journal of Child Language, 39(1), 61-89.

Khamis Dakwar, R., Ahmar, M., Farah, R., \& Froud, K. (2018). Diglossic aphasia and the adaptation of the Bilingual Aphasia Test to Palestinian Arabic and Modern Standard Arabic. Journal of Neurolinguistics, 47, 131-144.

Kiran, S. \& Roberts, P. (2012). What do we know about assessing language impairment in bilingual aphasia? In: Gitterman M, Goral M, Obler L, (Eds). Aspects of Multilingual Aphasia (35-50). Bristol: Multilingual Matters.

Paradis, M. \& Libben, G. (1987). The Assessment of Bilingual Aphasia. Hillsdale (NJ): Lawrence Earlbaum Associates.

Roberts, P. (2008). Issues in Assessment and Treatment for Bilingual and Culturally Diverse Patients. In Chapey, R. (ed), Language Intervention Strategies in Aphasia and Related Neurogenic Communication Disorders (245-275). Philadelphia: Lippincott Williams \& Wilkins. 


\title{
The Experiences of Aphasia Caregivers In Turkey
}

\author{
Hüsnünur ASLANTÜRK ${ }^{1}$, Nurten TİRYAKİ ${ }^{2}$ Bülent TOĞRAM ${ }^{3}$ \\ ${ }^{1}$ Department of Social Work, Kocaeli University, Kocaeli, Turkey \\ ${ }^{2}$ Department of Language and Speech Theraphy, İstanbul Medipol University, İstanbul, Turkey \\ ${ }^{3}$ Department of Language and Speech Theraphy, Eskişehir Anadolu University, Eskişehir, \\ Turkey
}

\begin{abstract}
Introduction
Aphasia has a profound effect on the patients and their primary caregivers. Being dependent on someone and impairment of ability to communicate are devastating and frustrating to both aphasic people and their caregivers. The caregivers face variety of challenges as a result of longterm caregiving responsibilities. Little is known about Turkish caregivers' own experiences as a result of care giving for aphasic relatives. The aim of this study was to reveal the Turkish caregivers' experiences during care giving.
\end{abstract}

\section{Methods}

Participants \& Data Collection

Semi-structured in-depth interviews were conducted with the eleven $(n=11)$ caregivers of adults with aphasia.

Data Analysis

All the interviews were audio-recorded and transcribed verbatim. The transcriptions of interviews were analyzed using qualitive content analysis via the program Nvivo11 to explore the key quotations and identify themes for the experiences of caregivers.

\section{Results}

Five core themes related to experiences of caregivers emerged from the analysis of transcripts. These core themes were; social isolation, communication problems, financial burdens, psychological burden of caregiving. The results revealed that caregivers were mostly impacted by language impairment in most areas in their lives. Due to being both a close relative and caregiver of people with aphasia, caregivers stated that they face with additional responsibilities as a result of role change in the family. The results also emphasized that aphasic people's mood changes affect whole family and relationships between caregivers and the person with aphasia.

\section{Discussion}

Caregivers' own experiences should be taken into consideration during the rehabilitation process of people with aphasia. During the language and speech therapy training the caregivers as a communication partner can enhance their relationship and communication with aphasic people whom they are giving care. Psychosocial support and support groups for both caregivers and people with aphasia can be beneficial to adapt their situation and the changes in their lives.

\section{References}

C. Code and M. Herrmann, "The relevance of emotional and psychosocial factors in aphasia to rehabilitation," Neuropsychological Rehabilitation, vol. 13, no. 1-2, pp. 109-132, 2003.

K. Hilari, J. J. Needle, and K. L. Harrison, "What are the important factors in healthrelated quality of life for people with aphasia? A systematic review," Archives of Physical Medicine and Rehabilitation, vol. 93, no. 1, supplement, pp. S86-S95, 2012.

Sjöqvist Nätterlund, B. (2010). Being a close relative of a person with aphasia. Scandinavian journal of occupational therapy, 17(1), 18-28. 
U. E. Coombs, "Spousal caregiving for stroke survivors," The Journal of Neuroscience Nursing, vol. 39, no. 2, pp. 112-119, 2007. 


\title{
Public Awareness of Aphasia in Turkey
}

\author{
Bülent Toğram \\ Center for Education, Research and Training Center for Speech \& Language Pathology \\ (DILKOM), Anadolu University, TURKEY
}

\section{Introduction}

The term aphasia is used to describe an acquired loss or impairment of the language system following brain damage (Benson \& Ardila, 1996). About one-third of all people who experience a stroke develop aphasia (Engelter, 2006). The literature implies that there is limited public awareness of aphasia in some countries, such as USA, England, Australia (Simmons Mackie, Code, Armstrong, Stiegler, \& Elman, 2002), Greece, Norway and Argentina (Code, Papathanasiou, Rubio-Bruno, Haaland-Johansen, de la Paz Cabana, \& Villanueva, 2009), Crotia (Leko \& Prizl-Jakovac, 2008) and Slovenia (Zemva). In Turkey, there is a study conducted with patients in a neurology practice (Mavis, 2007). However, the need for this study has emerged because of apply to relatively big sample using the same survey to compare with other awareness studies in literature. Therefore, the study aimed to explore current public awareness and knowledge of aphasia. It also investigated respondents' sources of information and whether demographic factors affected knowledge.

\section{Methods}

\section{Respondents}

The Awareness of Aphasia Survey (Simmons Mackie, et al., 2002) was adapted to Turkish and administered to Turkish people. A total of 673 people were surveyed. The survey were carried out by researcher using face-to-face interviews or online survey. All respondents were stratified by four age groups (14-44, 45-59, 60-74 and 75 years or older), four educational levels (1-5, $1-8,1-11$, and 12 years or more), and gender. Data were analysed to examine the number of individuals who had "heard of aphasia" and the number with "basic knowledge of aphasia". In addition, demographical characteristics of individuals were analysed.

\section{Tool and Procedure}

The Awareness of Aphasia Survey (Simmons Mackie, et al., 2002) was adapted to Turkish and administered to Turkish people. The survey was designed to be brief and simple in order to ensure maximum participation and cross cultural applicability. Respondents were asked basic demographic information consisting of age, education, gender, and occupation.

\section{Data Analysis}

Data were analysed to examine the number of individuals who had "heard of aphasia" and the number with "basic knowledge of aphasia". In addition, demographical characteristics of individuals were analysed.

\section{Results}

Preliminary data showed that awareness and knowledge about aphasia in Turkish people was low. The most of people who heard of aphasia had a "basic knowledge" of aphasia. There were significant demographical (age, gender and education) variations; however, all demographical variables demonstrated both limited awareness and knowledge of aphasia. Females were more likely to have heard of aphasia than males. Highly educated respondents were more likely to have heard of aphasia than lower educated respondents. The average age of respondents who had heard of aphasia was younger than those who had not heard of aphasia. There was a greater tendency for intermediate professionals to have heard of aphasia. People had heard 
of aphasia primarily through their work, the media, or through a relative or friend who had aphasia.

\section{Discussion}

Public knowledge and awareness may affect quality of delivered services, quality of life and acceptance of individuals with aphasia. Therefore, there are needed to improve public awareness of aphasia through kinds of campaigns (i.e, advocacy campaigns).

\section{References}

Benson, D.F. \& Ardila, A. (1996). Aphasia: a clinical perspective. New York: Oxford University Press.

Code, C., Papathanasiou, I., Rubio-Bruno, S., Haaland-Johansen, L. de la Paz Cabana, M. Villanueva, M.M. (2009). International Comparisons of the Public Awareness of Aphasia: Greece, Argentina and Norway. In Clinical Aphasiology Conference (2009: 39th: Keystone, CO: May 26-30, 2009).

Engelter, S.T., Gostynski, M., Papa, A., Frei, M., Born, C., Ajdacic- Gross, V., Gutzwiller, F., Lyrer, P.A. (2006). Epidemiology of Aphasia Attributable to First Ischemic Stroke: Incidence, Severity, Fluency, Etiology, and Thrombolysis. Stroke, 37, 1379-1384.

Leko, A. \& Prizl-Jakovac, T. (2008). Aphasia-What's that???. Nada Žemva \& Ilias Papathanasiou (Ed.), In 13th International Aphasia Rehabilitation Conference. (pp.72-72). Ljubljana: Institute for Rehabilitation Republic of Slovenia.

Mavis, I. (2007). Perspectives on public awareness of stroke and aphasia among Turkish patients in a neurology unit. Clinical Linguistics and Phonetics, 21(1), 55-70.

Simmons-Mackie, N., Code, C., Armstrong, E., Stiegler, \& Elman, (2002). What is aphasia? Results of an international survey. Aphasiology, 16, 837-848.

Zemva, N. Symposium "International Perspectives On The Public Awareness Of Aphasia": The Public Awareness Of Aphasia in Slovenia.

http://bib.irb.hr/datoteka/541786.Symposium.pdf 


\title{
The subject-object-verb word order as a self-cueing strategy in aphasia: An exploratory study
}

\author{
Svetlana Malyutina ${ }^{1,2}$, Yulia Akinina ${ }^{1,3}$, Valeriya Zelenkova ${ }^{1}$ \\ ${ }^{1}$ Center for Language and Brain, National Research University Higher School of Economics, \\ Moscow, Russia \\ ${ }^{2}$ Federal Center for Cerebrovascular Pathology and Stroke, Moscow, Russia \\ ${ }^{3}$ University of Groningen, Groningen, the Netherlands
}

\section{Introduction}

Numerous studies have demonstrated that people with aphasia (PWA) often produce noncanonical word orders in sentence production tasks (Saffran, Schwartz \& Marin, 1980). Interestingly, specific non-canonical word orders may not be produced with equal probability: some observations suggest that the subject-object-verb (SOV) order may be particularly prevalent. These observations come from English (Scholes, 1982), a language with a strict subject-verbobject (SVO) canonical order, and Russian (Akhutina, 1989, 2003), where word order is more flexible but SVO is still the most canonical (Baylin, 2011). To our knowledge, the prevalence of the SOV order has remained an observation, so our first aim is to independently verify it.

Our second aim is to better understand the mechanisms driving the use of the SOV order. Typically, it has been attributed to syntactic deficits: PWA substitute impaired surface syntax with unmarked word order (Scholes, 1982) or with "semantic syntax" reflecting the conceptual representation of the situation (Akhutina, 2003). However, we consider it debatable that SOV is necessarily the unmarked word order across languages and/or the best reflection of the conceptual representation. Thus, we believe that the syntactic account does not fully explain the prevalence of SOV over other non-canonical word orders.

We propose an alternative explanation: producing the subject and object may be a strategy to self-cue the production of the verb, which is typically the most cognitively demanding sentence element, by first retrieving its semantically related arguments. This account is in line with therapy approaches that explicitly use verb arguments to train verb retrieval within sentences (for example, Edmonds, Nadeau, \& Kiran, 2009). We test our account by complementing sentence production data with action naming data. We hypothesize that if SOV is a self-cueing strategy, (a) it will be used by PWA with verb naming deficits; (b) self-cueing will also be observed in the action naming task; (c) the use of SOV will lead to more successful verb retrieval at the item level.

\section{Methods}

We re-analyzed performance on transitive verbs $(\mathrm{N}=40)$ from a previously conducted experiment (Malyutina, Zelenkova \& Savcenko, 2018) testing verb argument structure effects across two tasks in 40 individuals with fluent $(\mathrm{N}=20)$ and non-fluent $(\mathrm{N}=20)$ post-stroke aphasia. In action naming, participants were asked to name a black-and-white picture of an action (Akinina et al., 2015) with one inflected verb. In sentence production, participants were shown the same pictures, provided with the verb and its arguments, and asked to build a grammatically correct sentence using all of these words, similarly to Cho-Reyes \& Thompson (2012).

To assess whether SOV was the most prevalent non-canonical word order, we manually tagged the word order in the sentence production task. For each participant, we calculated the proportions of specific word orders among responses that included all three sentence elements (subject, verb and object). 
To test our self-cueing strategy hypothesis, we used the naming accuracy from the same dataset as an indicator of verb retrieval difficulties. Also, we tagged the overt use of subjects and object in the action naming task, in non-compliance with the instructions, possibly as a self-cueing strategy. We then correlated the naming accuracy, the proportion of sentences with overt subjects and/or objects in the action naming task, and the proportion of SOV sentences in the sentence production task.

\section{Results}

Among non-canonical word orders including all three sentence elements (subject, verb, object), SOV was the most prevalent order at the group level (see Table 1). At the individual level, out of 18 participants who produced any non-canonical three-element orders, 13 participants produced the SOV order in over $50 \%$ of such responses.

Table 1. Proportions of specific word orders among all orders including all three sentence elements, mean (SD)

\begin{tabular}{lllllll}
\hline & SVO & SOV & VSO & VOS & OVS & OSV \\
\hline 'Non-fluent' aphasia $(\mathrm{N}=20)$ & $90(24) \%$ & $5(13) \%$ & $2(6) \%$ & $1(2) \%$ & $1(5) \%$ & $1(5) \%$ \\
'Fluent' aphasia $(\mathrm{N}=20)$ & $88(16) \%$ & $10(15) \%$ & $0(1) \%$ & $1(3) \%$ & $0(1) \%$ & $0(1) \%$ \\
\hline
\end{tabular}

The Spearman's rank correlation analysis yielded significant correlations between action naming accuracy and self-cueing in action naming, $r(38)=-.33, p=.04$; self-cueing in action naming and the proportion of SOV in sentence production, $r(38)=.46, p=.003$; and action naming accuracy and the proportion of SOV in sentence production, $r(38)=-.41, p=.008$.

\section{Discussion}

Our descriptive analysis confirms previous observations that SOV is the most prevalent noncanonical word order in Russian speakers with aphasia. The correlation analysis provides evidence in favor of the self-cueing nature of SOV: it is employed by individuals with poorer action naming abilities who also produce verb arguments in the action naming task, possibly also as a self-cueing strategy. However, because the verbs were provided in the sentence production task, these data did not allow to test one important component of the self-cueing strategy hypothesis: that SOV as a strategy leads to more efficient verb retrieval. This will be tested in another dataset: the Russian Verb and Sentence Test (Akinina \& Bastiaanse, 2017), which also includes both action naming and sentence production tasks, but in the latter, the verbs are not provided.

At this stage, our conclusions about the mechanisms driving the SOV prevalence are limited by the post-hoc nature of the analysis and should be tested in a controlled experiment. The final conclusions will have clinical implications for how the SOV word order is interpreted at diagnosis (i.e., whether it is interpreted as an indicator of syntactic and/or lexical difficulties) and whether it can be taught as a verb retrieval strategy during treatment, at least in languages with flexible word order such as Russian.

\section{References}

Akhutina, T. V. (1989). Porozhdenie rechi. Neyrolingvisticheckiy analiz sintaksisa [Language production: A neurolinguistic study of syntax]. Moscow: Moskovskiy Universitet [Moscow University].

Akhutina, T. V. (2003). The role of inner speech in the construction of an utterance. Journal of Russian and East European Psychology, 41(3-4), 49-74. 
Akinina, Y., \& Bastiaanse, R. (2017). Verb and sentence test in Russian: a showcase of two people with fluent aphasia. Stem-, Spraak- and Taalpathologie, 22(1), 134-136.

Akinina, Y., Malyutina, S., Ivanova, M., Iskra, E., Mannova, E., \& Dragoy O. (2015). Russian normative data for 375 action pictures and verbs. Behavior Research Methods, $47(3)$, 691-707.

Edmonds, L. A., Nadeau, S. E., \& Kiran, S. (2009). Effect of Verb Network Strengthening Treatment (VNeST) on lexical retrieval of content words in sentences in persons with aphasia. Aphasiology, 23(3), 402-424.

Bailyn, J. (2011). The Syntax of Russian (Cambridge Syntax Guides). Cambridge: Cambridge University Press.

Saffran, E. M., Schwartz, M. F., \& Marin, O. S. M. (1980). The word order problem in agrammatism: II. Production. Brain and Language, 10(2), 263-280.

Scholes, R. J. (1982). The verb-right strategy in agrammatic aphasia. Neuropsychologia, 20(3), 361-363. 


\title{
Differences in semantic priming during lifespan and in aphasia
}

\author{
Krethlow, G., Python, G., Laganaro, M.
}

\section{Introduction}

Word selection and production can be influenced by several psycholinguistics factors such as word frequency or age of acquisition (e.g. Alario et al., 2004; Newman \& German, 2005; Barry et al., 2006; Perret \& Bonin, 2018; Rabovsky et al., 2016), or by factors related to the semantic network (Meyer \& Schvaneveldt 1971; Ferrand \& New, 2003; McRae et al., 2012; Reilly \& Desai, 2017; Tree \& Hirsh, 2003; Gordon \& Cheimariou, 2013). Word production also undergoes changes during lifespan and therefore varies according to the age of the speaker (Kavé et al., 2010; Laganaro et al., 2015). The influence of the semantic network has an impact on word production across the lifespan, but this effect seems specific to the network of the age-group of the speaker (Krethlow et al., subm). As semantic relationships between words are used to interfere or facilitate word production both in healthy participants and in aphasia (Britt et al., 2016; Hashimoto, 2016; Python et al., 2018), one may wonder whether age-specific semantic relationships have larger influence on word production than age-independent associations.

In this study we investigate in healthy speakers and in patients with aphasia if the activation of the semantic network preceding an image to be named influences word production in different ways depending on whether the associates belong to the semantic network of the speaker's age-group or not.

\section{Methods}

Participants

Healthy participants have been recruited from two age-groups (corresponding to the age-groups from which we have semantic association data from Krethlow et al., subm), namely: 20 to 30 and 58 to 68 year-olds. Left hemisphere brain-damaged participants suffering from aphasia with mild to moderate anomia will also be recruited in the latter age-group (58-68 years-old).

\section{Material and procedure}

The task is a 23 items picture naming with auditory semantic priming/interference with a negative SOA (usually inducing a facilitation effect; e.g. Python et al., 2018). The primes preceding the picture to be named are either frequent semantic associates predominantly for the 20-30 age-group; frequent semantic associates predominantly for the 58-68 age-group or unrelated words.

For healthy subject the stimuli are presented by the E-Prime software (E-Studio). During the experimental trial, first a fixation cross is presented for $500 \mathrm{~ms}$ on the screen, then the prime word is heard and finally, after a fixed delay of $150 \mathrm{~ms}$, the picture appears for $2000 \mathrm{~ms}$ on a grey screen. Participants were asked to ignore the auditory word and overtly produce the word corresponding to the picture as fast and as accurately as possible. For aphasic patients the procedure is almost the same, but the picture appears for $5000 \mathrm{~ms}$ and the following trial is started by the experimenter.

All the responses were recorded with a microphone and digitized for accuracy and reaction time (RT) check.

\section{Preliminary Results}

Data collection is currently underway. Preliminary results on 18 young-adult speakers (20-30 years-old) show unexpectedly significantly longer RTs for pictures preceded by frequent associates for 20-30 group (776 ms) relative to unrelated primes (730 ms) and intermediate RTs for 
primes which are frequent for the other age-group (764 ms).

A 59 year-old participant suffering from aphasia with lexical-semantic anomia showed the largest interference on accuracy for items preceded by semantic associates specific to the 58-68 group relative to unrelated and to items preceded by associates from the 20-30 age-group.

\section{Discussion}

The preliminary results show that semantic associates presented before the picture to be named causes interference rather than the expected facilitation in both healthy participants and a participant with aphasia. This was all the more the case when the associates were issued from the age-specific group of the participants. The results thus indicate that activating the semantic network specific to the age-group of the speaker seems to interfere with word production on top of a general (unspecific) semantic network. These results have to be confirmed with healthy participants in the other age-group (58-68) and with a larger group of speakers with aphasia.

\section{References}

Alario, F. X., Ferrand, L., Laganaro, M., New, B., Frauenfelder, U. H., \& Segui, J. (2004). Predictors of picture naming speed. Behavior Research Methods, Instruments, \& Computers, 36(1), 140-155.

Barry, C., Johnston, R. A., \& Wood, R. F. (2006). Effects of age of acquisition, age, and repetition priming on object naming. Visual cognition, 13(7-8), 911-927.

Britt, A. E., Ferrara, C., \& Mirman, D. (2016). Distinct Effects of Lexical and Semantic Competition during Picture Naming in Younger Adults, Older Adults, and People with Aphasia. Frontiers in Psychology, 7, 813.

Ferrand, L., \& New, B. (2003). Semantic and associative priming in the mental lexicon. Mental lexicon: Some words to talk about words, 25-43.

Gordon, J. K., \& Cheimariou, S. (2013). Semantic interference in a randomized naming task: Effects of age, order, and category. Cognitive neuropsychology, 30(7-8), 476-494.

Hashimoto, N. (2016). The use of one or three semantic associative primes in treating anomia in aphasia. American journal of speech-language pathology, 25(4S), S665-S686.

Kavé, G., Knafo, A., \& Gilboa, A. (2010). The rise and fall of word retrieval across the lifespan. Psychology and Aging, 25(3), 719.

Laganaro, M., Tzieropoulos, H., Frauenfelder, U. H., Zesiger, P., 2015. Functional and timecourse changes in single word production from childhood to adulthood. NeuroImage, 111, $204-214$.

McRae, K., Khalkhali, S., \& Hare, M. (2012). Semantic and associative relations in Adolescents and Young Adults: Examining a tenuous dichotomy. In V. F. Reyna, S. B. Chapman, M. R. Dougherty, \& J. Confrey (Eds.), The Adolescent Brain: Learning, Reasoning, and Decision Making (pp. 39-66). Washington, DC: APA.

Meyer, D. E., \& Schvaneveldt, R. W. (1971). Facilitation in recognizing pairs of words: evidence of a dependence between retrieval operations. Journal of experimental psychology, 90(2), 227.

Newman, R. S., \& German, D. J. (2005). Life span effects of lexical factors on oral naming. Language and Speech, 48(2), 123-156.

Perret, C., \& Bonin, P. (2018). Which variables should be controlled for to investigate picture naming in adults? A Bayesian meta-analysis. Behavior research methods, 1-13.

Python, G., Fargier, R., \& Laganaro, M. (2018). When wine and apple both help the production of grapes: ERP evidence for post-lexical semantic facilitation in picture naming. Frontiers in human neuroscience, 12, 136.

Python, G., Glize, B., Laganaro, M. (2018). The involvement of left inferior frontal and middle temporal cortices in word production unveiled by greater facilitation effects following 
brain damage. Neuropsychologia, 121, 122-134. doi: 10.1016/j.neuropsychologia.2018.10.026. Rabovsky, M., Schad, D. J., \& Rahman, R. A. (2016). Language production is facilitated by semantic richness but inhibited by semantic density: Evidence from picture naming. Cognition, 146, 240-244.

Reilly, M., \& Desai, R. H. (2017). Effects of semantic neighborhood density in abstract and concrete words. Cognition, 169, 46-53.

Tree, J. J., \& Hirsh, K. W. (2003). Sometimes faster, sometimes slower: Associative and competitor priming in picture naming with young and elderly participants. Journal of Neurolinguistics, 16(6), 489-514. 


\title{
Speech and language therapy for acquired dysgraphia in neurological patients: a systematic review
}

\author{
Brisotto C., Biddau F., Nordio S. \\ IRCCS San Camillo Hospital, Venice, Italy
}

\section{Introduction}

Acquired dysgraphia refers to a disorder of linguistic and cognitive aspect of writing and spelling (Weekes, 2015) and usually co-occurs with impairments to other language modalities (reading, spoken word retrieval, auditory comprehension), in people suffering from aphasia (Damasio, 1998). Oral language is dominant in everyday communication (Nickels, 2002), therefore rehabilitation of spoken language is often considered a priority over written language treatment in clinical management of aphasia. Nevertheless, written language may be easier to improve and it could be a useful strategy to support oral communication in aphasic patients (Rapcsak \& Beeson, 2002). Moreover, it is important to highlight the role of written communication in everyday life, work situations and social interactions, considering e.g. the increasing use of mobile phones, tablet and personal computer.

A recent narrative review (Thiel, 2015) found that dysgraphia therapy studies have been mostly focused on single-word spelling then on training written language for real life situations. Therefore, we believe that it could be essential to summarize the main evidence based methods of treatments to improve written language abilities.

The aim of this systematic review is to verify the effectiveness of speech and language therapy (SLT) for acquired dysgraphia due to neurological diseases in acute or post-acute phase, in terms of improvement of i) written lexical access, ii) phonological procedure and iii) spelling of written words or sentences.

\section{Methods}

Search strategy and selection criteria

The review was registered in PROSPERO (International Prospective register of systematic reviews) with the registration number CRD42018084221. We used the PRISMA 2009 checklist (Moher D, Shamseer L, Clarke M, et al, 2015) to establish the protocol.

Systematic searches of four key electronic databases were conducted until December 2018 using key words such as dysgraphia, agraphia, written language, writing impairment, speech therapy, intervention, treatment, rehabilitation.

Eligible studies were identified according to selection criteria: they had to test the efficacy of speech and language therapy for the recovery of written language impairments in patients affected by dysgraphia due to neurological diseases; authors excluded studies on patients with writing impairments due to dementia, cancer and progressive diseases. Moreover, were included only randomized controlled trials (RCT), controlled trials (CT), case series, single-subject design (SSD) and controlled before-after (CBA) studies. Finally, studies were required to be published in English.

\section{Data collection and analysis}

Two authors independently screened the titles and abstracts of the records retrieved from the search strategy, applying the selection criteria previously described.

The full text of the possible eligible records were retrieved and analyzed for final inclusion in this systematic review. Three authors independently extracted data from each record and tabulated studies characteristics.

Methodological quality was assessed using the Downs and Black checklist (1998) by two au- 
thors. Levels of evidence ratings were assigned using the Oxford Centre for Evidence based Medicine's (OCEBM) Levels of Evidence 1 (2009).

Owing to the heterogeneity of included studies in their design, population, type of treatment and data collection and analyses, a narrative synthesis was conducted.

\section{Results}

Database searches identified 447 study titles. 76 duplicates were removed, leaving 371 titles and abstracts to be screened. Of these, 19 abstracts were selected for full-text reading; then, 10 studies were included in the final review and analyzed through.

\section{PRISMA 2009 Flow Diagram}
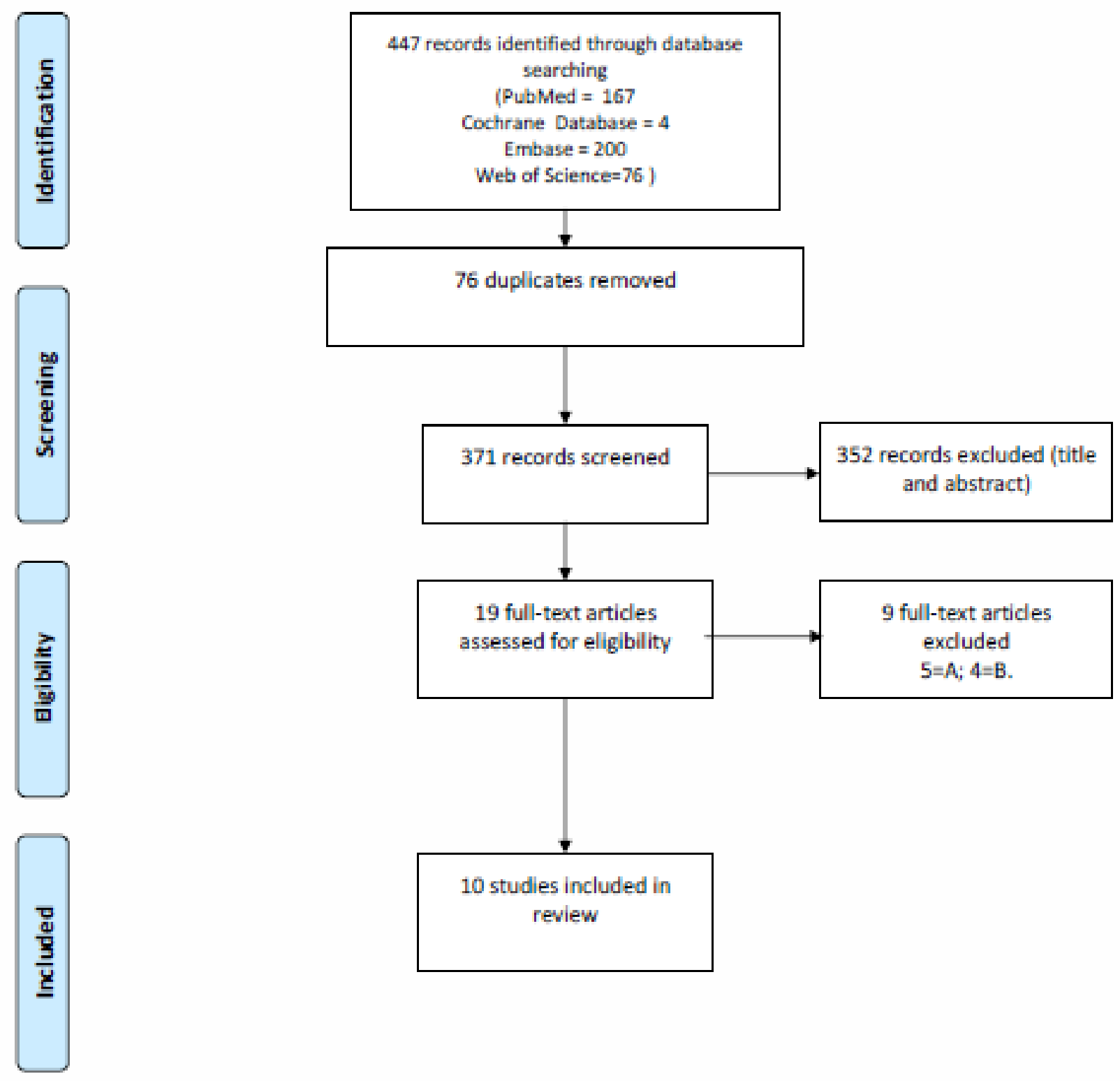

Figure 1. Flow diagram outlining the study's selection process. Rejection criteria: $A=$ inappropriate aim; $\mathrm{B}=$ study design not specified. 
All the papers included in the review had a low level of evidence, rated as level 4 according to the OCEBM's Levels of Evidence 1 (2009).

Methodological quality assessment through the application of the Downs and Black checklist (1998), identified flaws and risk of bias in all studies. External and internal validity were particularly critic: papers didn't report recruitment procedures and blinding of participants and assessors; moreover, there was lack of randomization and of investigation of the main confounders. On the other hand, all studies clearly described case reports, the aim and the interventions of interest and the most of them clearly described also the main findings. Moreover, according to the authors, studies applied appropriate statistical analysis of their results except for one that reported only raw scores.

Preliminary narrative synthesis found that all the studies had a small sample size that ranged from 2 to 8 participants per study; participants had a homogeneous etiology and most of them were in a chronic phase of the disease. Only two studies recruited patients in acute phase. Types of aphasia and dysgraphia, instead, are heterogeneous in every study.

Regarding the outcomes measured, most of the studies investigated spelling accuracy of trained and untrained words after the intervention. Some studies also investigated oral naming and spelling accuracy, reading rate and accuracy, speed of spelling, sound-letter and letter-sound correspondences. Treatments were all administrated during individual sessions; in most of the studies participants were also requested to do daily homework and just in one paper a weekly group sessions were administrated. Frequency of therapies varied from one to three times per week. The treatments' duration lasted from 4 to 14 weeks. Types of treatment were heterogeneous and could be administrated combined or not: lexical therapies (i.e., whole word writing), phonological therapies (focusing on sound-to-letter correspondences), interactive therapies (involving also problem solving strategies) errorless therapies (designed to minimize errors) and errorful therapies (with progressive orthographic cues).

\section{Discussion}

From an overall synthesis of the preliminary results, it seems that the systematic review did not provide final evidence that any therapy approach is more effective than any other in dysgraphia recovery.

Nevertheless, the majority of studies, predominantly focused on single word spelling accuracy, shown that participants with a range of linguistic and spelling abilities can achieve positive gains following therapy, as already highlighted by Thiel et al., 2015. Improvements were reached despite differences in linguistic characteristics and level of severity and despite the different frequency and duration of treatments.

Future trials need to have a more robust design and evaluate the effectiveness of treatments based on common outcomes.

\section{References}

Weekes, B.S. (2005). Acquired disorders of reading and writing: Cross-script comparisons. Behavioural Neurology, 16(2,3), 51-57.

Damasio, A. R. (1998). Signs of aphasia. Acquired aphasia, 2, 27-43.

Nickels, L. (2002). Theoretical and methodological issues in the cognitive neuropsycology of spoken word production. Aphasiology, 16 (1/2), 3-9.

Rapcsak, S., Beeson, P.M. (2002). Agraphia. Enciclopedia of the Human Brain, 1, 71-86

Thiel, L., Sage, K., Conroy, P., (2015) Retraining writing for functional purposes: a review of the writing therapy literature. Aphasiology, 29:4, 423-441.

Moher, D., Shamseer, L., Clarke, M., Ghersi, D., Liberati, A., Petticrew, M., ... \& Stewart, L. A. (2015). Preferred reporting items for systematic review and meta-analysis protocols 
(PRISMA-P) 2015 statement. Systematic reviews, 4(1), 1.

Downs, S.H., Black, N., (1998). The feasibility of creating a checklist for the assessment of the methodological quality both of randomised and non-randomised studies of health care interventions. Epidemiol Community Health, 52, 377-384.

OCEMB Levels of Evidence Working Group. (2009). The Oxford 2009 levels of evidence. 


\title{
Neurofunctional correlates of auditory and visual sentence comprehension: Evidence from aphasia
}

\author{
Sabrina Beber ${ }^{1}$, Rita Capasso ${ }^{2}$, Gabriele Miceli ${ }^{1,3}$ \\ ${ }^{1}$ CIMeC (Centro interdisciplinare Mente/Cervello), Università di Trento \\ ${ }^{2}$ Brain Associates, Roma \\ ${ }^{3}$ Centro Linceo Interdisciplinare 'Beniamino Segre', Accademia dei Lincei, Roma
}

\section{Introduction}

Several studies have recently focused on the neural correlates of sentence comprehension disorders in aphasia (Thothathiri, Kimberly \& Schwartz, 2011; Magnusdottir et al, 2013; Rogalsky et al, 2017; den Ouden et al, 2019; Walenski, Europa, Caplan, Thompson, 2019). Results challenged the traditional view that prefrontal regions are essential for sentence comprehension. Investigations largely converge in assigning a critical role to parieto-temporal regions; they acknowledge less consistently a role for anterior/superior temporal areas and only occasionally attribute a relevant role to left prefrontal regions.

Despite their unquestionable interest, these studies focus on the neural correlates of auditory comprehension but leave unexplored those of written sentence comprehension. As a consequence, it is not clear if the areas identified in these studies are involved in modality-specific or modality-neutral processes.

We investigated the neurofunctional correlates of sentence comprehension in 26 individuals with aphasia, in order to establish whether the same brain areas are critical for auditory and visual sentence processing, and identify the regions involved in modality-specific and modality-neutral comprehension.

\section{Methods}

54 participants with aphasia presenting sentence comprehension deficits and 90 healthy volunteers completed an auditory and a visual sentence/picture matching task. All sentences were semantically reversible. Both tasks included an identical number of active and passive stimuli (La bambina abbraccia la mamma, The girl hugs the mother; La mamma è abbracciata dalla bambina, The mother is hugged by the girl). Participants heard or read a sentence and at the same time saw two vertically aligned, black-and-white drawings. They were asked to select the drawing that corresponded to the sentence. In each item, a drawing matched the stimulus, and the other was a thematic role foil, a morphological foil or a lexical-semantic foil (one-third each). The auditory task included 60 stimuli, the visual task 45.

Twenty-six participants with aphasia who made no errors on semantic foils but scored $>2$ sd below norm on other foil types in the auditory or visual task were retained. Of these, 8 scored below norm only in the auditory task (Auditory group), 9 only on the written task (Visual group) and 9 on both tasks (Auditory-Visual group). Lesions were reconstructed in FSL (https://fsl.fmrib.ox.ac.uk/fsl/fslwiki/) based on MRI or high-quality CT-scan images. Correlations between brain structures and behavioral variables were analyzed by VLSM (Bates et al, 2003). The analyses were conducted on voxels damaged in at least $10 \%$ of the sample.

\section{Results}

Brain damage is distributed differently in the three groups.

Lesions in the Auditory group overlap in prefrontal and perisylvian regions. Prefrontal damage is observed only in this group. It affects the inferior frontal gyrus (pars opercularis and triangularis), the insula, the frontal orbital cortex, and the frontal and central opercula. Additional perisylvian damage involves a small portion of the planum polare, Heschl's gyrus and the parietal operculum, and extends posteriorly to a small portion of the supramarginal gyrus. 
Lesions in the Visual group mostly overlap in temporo-parietal regions, rarely involved in the Auditory group (the temporo-occipital division of the middle temporal gyrus, the angular gyrus, the planum temporale). They extend to the parietal and central opercula, the planum polare, the Heschl's gyrus and the insula.

In the Auditory-Visual group, damage overlaps in a posterior temporo-parietal area including the supramarginal gyrus (posterior division), the angular gyrus, the planum temporale, the lateral occipital cortex (superior division) and a small temporooccipital-posterior portion of the middle temporal gyrus.

Collapsing data across the three groups, a Brunner-Munzel test was performed to identify the regions associated with the lowest accuracy in the auditory task, in the visual task, and in both tasks $(p<.05$ for all comparisons; Figure 1). Results showed low scores in auditory comprehension to be significantly associated with damage to the inferior frontal gyrus (pars opercularis and triangularis), the insula and the frontal orbital cortex. Lower accuracy in visual comprehension was associated with damage to the angular and the supramarginal gyrus, the temporo-occipital portion of the middle temporal gyrus and part of the lateral occipital cortex. Poorer performance on both comprehension tasks was significantly associated with damage to the angular gyrus and part of the superior lateral occipital cortex.

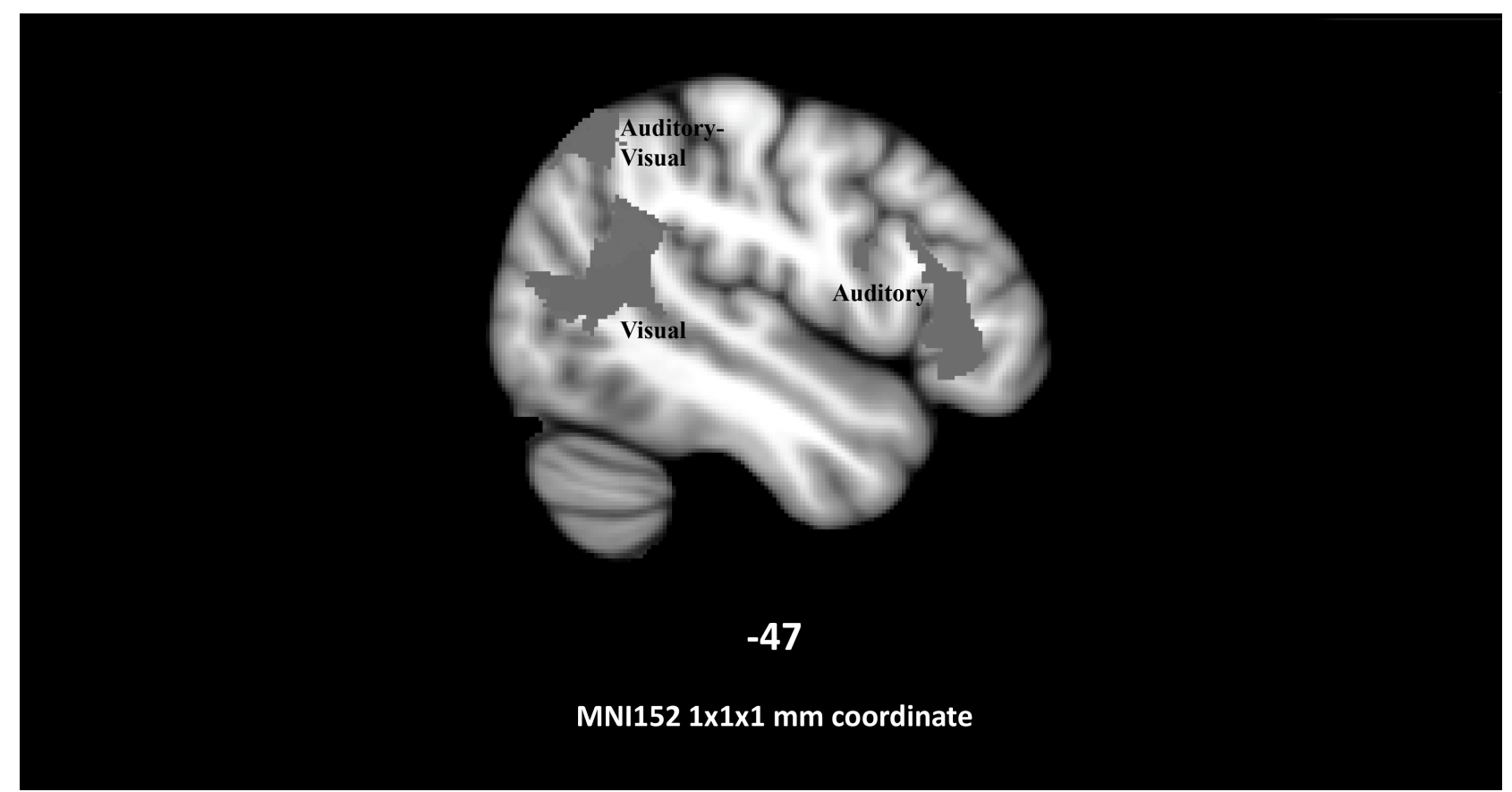

Figure 1. Areas associated with poor performance on the Auditory task, on the Visual task, and on both the Auditory and the Visual task (Brunner-Munzel test; $p<.05$ ).

\section{Discussion}

We studied the neural correlates of auditory and visual sentence comprehension in 26 individuals with aphasia. Distinct patterns of neural impairment were observed. Poor auditory comprehension was associated with damage to prefrontal regions, centered around the inferior frontal gyrus. Poor visual comprehension occurred as a consequence of inferior parietal and posterior temporal damage. Interestingly, the insula and Heschl's gyrus were lesioned in subjects from both groups (poor sentence comprehension in these cases might result from damage to fiber tracts underlying these structures, which may have disconnected critical cortical areas). Modality-independent comprehension difficulties were associated with damage to the posterior portion of the superior parietal lobule.

Poor auditory sentence comprehension following inferior frontal gyrus damage could be due 
to the role of this area in the phonological loop component of auditory-verbal working memory (Romero Lauro et al, 2010). The correlation of posterior temporal damage with poor visual sentence comprehension is less obvious, but the involvement of this region in reading has been documented repeatedly (Price, 2012). Damage to the superior parietal lobule (angular and supramarginal gyrus) correlates with modality-independent sentence comprehension difficulties, possibly due to its role in the re-analysis of thematic roles in reversible sentences (Finocchiaro et al, 2015).

\section{References}

Bates E., Wilson S.M., Saygin A.P., Dick F., Sereno M.I., Knight R.T., \& Dronkers N.F. (2003). Voxel-based lesion-symptom mapping. Nat Neurosci, 6(5), 448-450.

Den Ouden D.B., Malyutina S., Basilakos A., Bonilha L., Gleichgerrcht E., Yourganov G., Hillis A.E., Hickok G., Rorden C., \& Fridriksson J. (2019) Cortical and structuralconnectivity damage correlated with impaired syntactic processing in aphasia. Hum Brain Mapp doi: 10.1002/hbm.24514.

Finocchiaro C., Capasso R., Cattaneo L., Zuanazzi A. \& Miceli, G. (2015). Thematic Role Assignment in the Posterior Parietal Cortex: A TMS study. Neuropsychologia, 77:223232 .

Jenkinson M., Beckmann C.F., Behrens T.E., Woolrich M.W., \& Smith S.M. (2012). Fsl. Neuroimage, 62(2), 782-790.

Magnusdottír S., Fillmore P., den Ouden D.B., Hjaltason H., Rorden C., Kjartansson O., Bonilha L. \& Fridriksson J. (2013). Damage to left anterior temporal cortex predicts impairment of complex syntactic processing: A lesion-symptom mapping study. Hum Brain Mapp, 34:2715-2723.

Price C.J. (2012). A review and synthesis of the first 20 years of PET and fMRI studies of heard speech, spoken language and reading. NeuroImage, 62, 816-847.

Rogalsky C., LaCroix A.N., Chen K.-H., Anderson S.W., Damasio H., Love T. \& Hickok G. (2017). The neurobiology of agrammatic sentence comprehension: A lesion study. J Cogn Neurosci, 30:2, pp. 234-255.

Romero Lauro L.J., Reis J., Cohen L.G., Cecchetto C., \& Papagno C. (2010). A case for the involvement of phonological loop in sentence comprehension. Neuropsychologia, 48 , 4003-4011.

Thothathiri M., Kimberg D.Y. \& Schwartz M.F. (2011). The neural basis of reversible sentence comprehension: Evidence from voxel-based lesion symptom mapping in aphasia. $J$ Cogn Neurosci, 24:1, 212-222.

Walenski M., Europa E., Caplan D. \& Thompson C.K. (2019). Neural networks for sentence comprehension and production: An ALE-based meta-analysis of neuroimaging studies. Hum Brain Mapp, DOI: 10.1002/hbm.24523. 


\section{Characterising the Nature of Discourse Treatment in Aphasia Rehabilitation Research}

Lucy Dipper $^{1}$, Madeline Cruice ${ }^{1}$, Jane Marshall ${ }^{1}$, Nicola Botting ${ }^{1}$, Mary Boyle $^{2}$, Deborah Hersh $^{3}$, Madeleine Pritchard ${ }^{1}$

${ }^{1}$ City, University of London, UK

${ }^{2}$ Montclair State University, USA

${ }^{3}$ Edith Cowan University, Australia

\section{Introduction}

Discourse treatment is a priority for people with aphasia (PWA) and their family members [1], as well as for aphasia clinicians and managers [2]. Discourse treatment is correspondingly recommended in aphasia rehabilitation $[3,4]$. However, this is a relatively new field in treatment research and in practice, and there is no clear overview of the evidence base to inform the future research agenda or guide clinical practice. In this paper, we report on a scoping review of discourse treatment in aphasia rehabilitation, with the purpose of characterising the nature of therapies provided.

\section{Methods}

Scopus, Medline, and EmBase databases were searched on 25/05/18, using the terms ['discourse' or 'narrative' or 'story' or 'storytelling' or 'connected speech'] and ['intervention' or 'treatment' or 'therapy'] and ['aphasia' or 'dysphasia']. A total of 333 records were identified via database $(n=330)$ and hand searching $(n=3)$. Studies were included if they reported clients with aphasia following LH stroke; were published in a peer reviewed journal; reported primary data; were published in English; reported direct intervention with clients; and included discourse as a component of assessment and intervention. The therapy reported in each paper was extracted and scrutinized to determine the specific target of treatment within discourse, and studies were thus categorized: single word (e.g., a study focused on improving adjective production within discourse was categorized as single word), sentence, discourse, scripting, or multi-level (2 or 3 levels i.e. word, sentence and discourse). Treatment within studies was categorized by 3 members of the research team and then discussed.

\section{Results}

Twenty-five (25) studies were included in the review, reporting a total of 132 participants, published between 1991 and 2018. There was complete agreement for $65 \%$ of papers on first coding, with majority agreement (amongst 5 teams members) for $88 \%$ of papers. To reach further consensus we discussed which activities described in each paper were therapeutic, and agreed that there had to be an explicit statement that cueing, correction, feedback or scaffolding was provided by the clinician for that activity, in order to be included as a 'therapeutic activity' which could then be coded. Disagreements were re-considered on this basis, and consensus thereby reached for 23 papers (92\%). No consensus was reached on the final two papers. Single word treatment studies ( 5 studies; $\mathrm{n}=12$ participants) used Semantic Feature Analysis, group therapies for word production, and phonological and orthographic cuing therapies. Sentence level treatment studies ( 5 studies; $\mathrm{n}=55$ ) used Treatment of Underlying Forms, mapping therapy, modified response elaboration therapy, training verb production, and oral reading therapies. Discourse treatment studies ( 2 studies; $\mathrm{n}=5$ ) focused on improving discourse through exercises and storytelling. Scripting treatment studies (3 studies; $\mathrm{n}=22$ ) used AphasiaScripts ${ }^{\mathrm{TM}}$ ther- $^{-}$ apy. Multilevel (two level) treatment studies ( 5 studies; $n=16$ ) focused on training adjectives and discourse production, PACE therapy and discourse production, informativeness and/or morpho-syntactic organisation of connected speech, and sentence and discourse. Multilevel (3 
level) treatment studies ( 3 studies; $\mathrm{n}=22$ ) focused on improving single words, utterances, and the organisation of discourse, single word production and error analysis in discourse, sentence or utterance production in discourse, and macrostructural elements in discourse. Finally, for the two studies where consensus could not be reached, therapies used technology to improve spoken discourse (AAC device and Sentence Shaper software).

\section{Discussion}

The discourse treatment evidence base is an emerging field which subsumes a diverse number of distinct therapy approaches, some of which are highly recognizable. The variety in treatment targets has implications for synthesizing the evidence base in terms of efficacy and outcome measurement comparisons. The familiarity of the treatments used by clinical researchers in discourse treatment should encourage adoption by practising clinicians, but the number of different approaches might be a barrier to choosing a single one. More multi-level and discourse treatment research is needed.

\section{References}

1 Wallace, S.J., Worrall, L., Rose, T., Le Dorze, G., Cruice, M., Isaksen, J., Kong, A.P.H., Simmons-Mackie, N., Scarinci, N. and Gauvreau, C.A., 2017. Which outcomes are most important to people with aphasia and their families? An international nominal group technique study framed within the ICF. Disability and rehabilitation, 39(14), pp.13641379.

2 Wallace, S. J., Worrall, L., Rose, T., \& Le Dorze, G. (2017). Which treatment outcomes are most important to aphasia clinicians and managers? An international e-Delphi consensus study. Aphasiology, 31(6), 643-673.

3 Clinical Centre for Research Excellence in Aphasia Rehabilitation. (2014). Aphasia Rehabilitation Best Practice Statements 2014 comprehensive supplement to the Australian Aphasia Rehabilitation Pathway. Brisbane: CCRE in Aphasia Rehabilitation.

4 Hebert, D., Lindsay, M. P., McIntyre, A., Kirton, A., Rumney, P. G., Bagg, S., Bayley, M., Dowlatshahi, D., Dukelow, S., Garnhum, M. \& Glasser, E. (2016). Canadian stroke best practice recommendations: stroke rehabilitation practice guidelines, update 2015 . International Journal of Stroke, 11(4), 459-484. 


\title{
In Time with the Beat: Entrainment in Patients with Phonological Impairment, Apraxia of speech and Parkinson's disease
}

\author{
Ingrid Aichert ${ }^{1}$, Katharina Lehner ${ }^{1}$, Simone Falk ${ }^{2}$, Mona Späth $^{3}$, Mona Franke ${ }^{1} \&$ Wolfram \\ Ziegler $^{1}$ \\ ${ }^{1}$ Clinical Neuropsychology Research Group (EKN), Institute of Phonetics and Speech \\ Processing, Ludwig-Maximilians-Universität, Munich, Germany \\ ${ }^{2}$ Laboratoire Phonétique et Phonologie, Université Sorbonne Nouvelle Paris-3, Frankreich \\ ${ }^{3}$ neolexon, Munich
}

\section{Introduction}

Earlier studies have shown that patients with neurogenic speech sound impairments benefit from auditory stimulation by regular speech rhythms. An auditory priming experiment reported by Späth et al. (2016) revealed that speech initiation in individuals with Parkinson's (PD) was facilitated by a perceived regular speech rhythm. A recent study by Aichert et al. (in press) showed an influence of the metrical regularity of speech input in patients with apraxia of speech (AOS) and in patients with phonological impairment (PI). The two patient groups produced fewer segmental errors on target words preceded by regularly stressed prime sentences as compared to prime sentences with an irregular metrical pattern. They therefore seemed to exploit rhythmic cues in others' speech for their own production.

In the present study we investigated if patients with PI, AOS and PD are able to accommodate their speech to the natural speech rhythm of an auditory model and, if so, whether the effect is larger after hearing sentences with a metrically regular as compared to an irregular pattern. This question builds on theories of rhythmic entrainment, assuming that sensori-motor predictions of upcoming events allow humans to synchronize their actions with an external rhythm (e.g., Repp, 2005). Moreover, rhythm-based methods proved successful in treatment trials of AOS (e.g., Wambaugh \& Martinez, 2000) and dysarthria (Thaut et al. 2001).

\section{Methods}

Participants

Three groups of monolingual German-speaking patients participated in the study:

- 12 patients with apraxia of speech (AOS) and coexisting aphasia,

- 12 participants with aphasic phonological impairments (PI) without apraxia of speech, and

- 12 individuals with Parkinson's dysarthria (PD).

Furthermore, 24 neurologically healthy speakers were examined.

\section{Procedure and Materials}

A sequential synchronization paradigm based on a spoken sentence completion task was conducted. The materials comprised 96 sentences consisting of five two-syllabic words each. Regular prime sentences contained a series of four trochaic words (strictly alternating strong-weak pattern), whereas irregular prime sentences consisted of an alternation of trochaic and iambic words (trochee-iamb-iamb-trochee). Target words to be filled in by participants after a prime were either trochees (regular) or iambs (irregular). There were four conditions:

(1) Regular prime, trochaic target, e.g., 'Lena 'pflanzte 'damals 'diese 'Tulpe.

(2) Regular prime, iambic target, e.g., 'Friedrich 'neckte 'häufig 'diesen Te'nor

(3) Irregular prime, trochaic target, e.g., 'Jule ver'schenkt je'doch 'diese 'Tulpe.

(4) Irregular prime, iambic target, e.g., 'Mira be'trog zu'nächst 'diesen Te'nor.

The experiment was implemented in PsychoPy. Sentences were presented in a randomized order. 


\section{Data Analysis}

Response latencies (intervals between the onset of the last word of the prime sentence and the onset of the spoken target word) were measured in Praat. Words with phonological errors on the first syllable and responses preceded by groping behavior or by latencies more than 2000ms were discarded. To relate the response latencies to the spoken rhythm of the prime, we determined the average foot durations of the model speaker's prime sentences.

Linear mixed effects models were calculated to estimate the effects of prime regularity (factor PRIME) and target regularity (factor TARGET) on response latencies in the four groups (factor GROUP), with ITEMS as random intercepts and SUBJECTS as random slopes.

\section{Results}

Across the 96 prime sentences, mean foot durations were virtually identical in the regular (654ms) and the irregular $(655 \mathrm{~ms})$ condition. The figure depicts the deviation of a speaker's response latency from the latency value predicted by the metrical rhythm of the preceding prime. Values of 0 correspond to initiation delays equaling the average foot duration of the respective prime. Negative and positive values represent speech initiation delays shorter or longer than this unit.

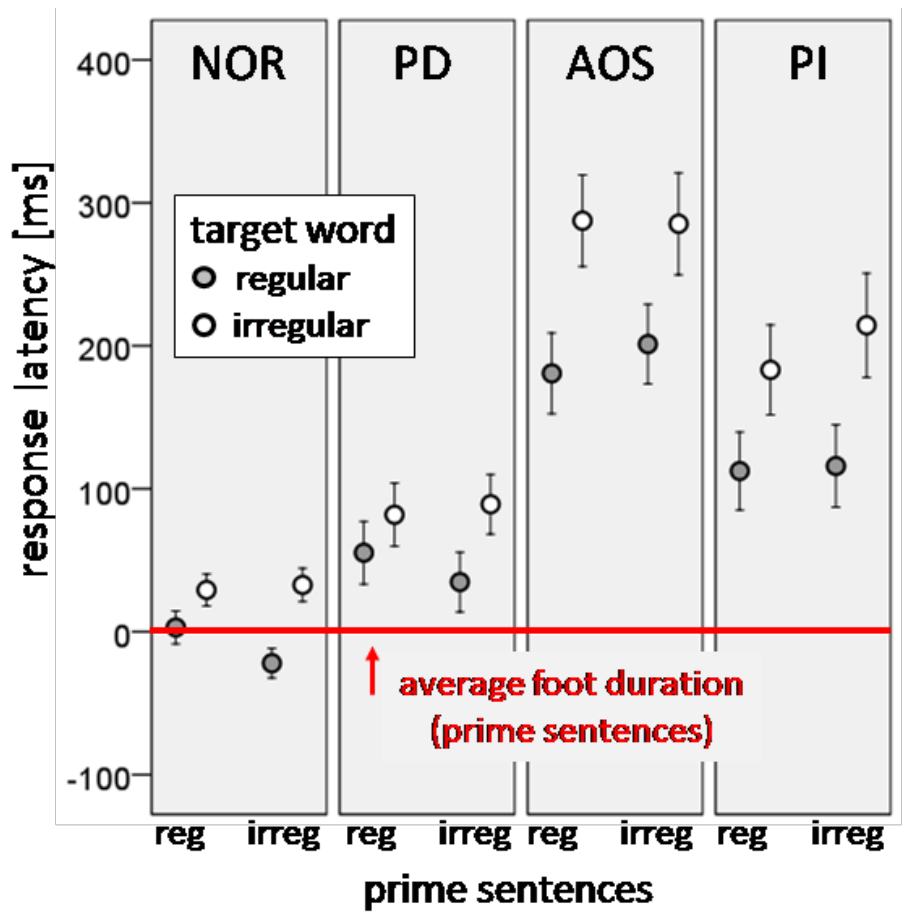

Figure 1. Adjusted response latency (ms) as a function of prime and target regularity for the patient groups (PD, AOS, PI) and the healthy control speakers (NOR).

Likelihood-ratio tests revealed significant effects of the factors GROUP $(\mathrm{F}(3,55)=11.3, p<.001)$ and TARGET $(\mathrm{F}(1,53)=15.1, p<.001)$, but not of PRIME $(\mathrm{F}(1,50)=0.12$, n.s. $)$. Furthermore, among the two-way interactions, significant effects were observed for GROUP $\times$ PRIME $(\mathrm{F}(3,44)=3.9, p<.05)$ and for GROUP $\times \operatorname{TARGET}(\mathrm{F}(3,44)=5.9, p<.01)$. Analyses restricted to the healthy speakers showed significant effects of PRIME $(\mathrm{F}(1,356)=8.3, p<.01)$ and TARGET $(\mathrm{F}(1,47)=7.6, p<.01)$ and a significant interaction of PRIME $\mathrm{x}$ TARGET $(\mathrm{F}(1,2197)=10.8, p<.01)$. 


\section{Discussion}

Healthy speakers demonstrated perfect entrainment of trochaic target words to the rhythm of regularly stressed prime sentences, delaying their responses almost exactly by the average foot duration of the preceding prime. In contrast, trochaic target words following irregular prime sentences were produced faster than expected. In this condition, a temporal reference frame could not be set up to establish rhythmical expectations for the subsequent target word production. Irregular target words were produced slower in both conditions.

Even though slower across all conditions, Parkinson's patients (PD) showed a similar pattern between conditions. This finding confirms the results of Späth et al. (2016) where patients proved to be able to entrain their speech rhythm with another person's speech, especially in a metrically regular condition. However, considerably longer response latencies with differing patterns between conditions were observed for the AOS and PI patients. Irregular prime sentences caused only a slight increase of latencies. The absence of regularity-dependent entrainment effects in these patients may be explained by their more severe difficulties in initiating speech at all. However, both patient groups showed a strong effect of target regularity. Irregular (iambic) words were initiated with considerable longer latencies compared to regular (trochaic) words. This result is in agreement with the error data reported from the same experiment (Aichert et al., in press). Moreover, it confirms earlier results based on repetition tasks, in which the regular lexical stress pattern of German (the trochee) facilitated articulation in patients with AOS (e.g., Aichert et al., 2016).

\section{References}

Aichert, I., Lehner, K.,Falk, K., Späth, M. \& Ziegler, W. (in press). Do patients with neurogenic speech sound impairments benefit from auditory priming with a regular metrical pattern? Journal of Speech, Language and Hearing Research

Aichert, I., Späth, M. \& Ziegler, W. (2016). The role of metrical information in apraxia of speech: Perceptual and acoustic analyses of word stress. Neuropsychologia, 82, 171-178.

Repp, B. H. (2005). Sensorimotor synchronization: A review of the tapping literature. Psychonomic Bulletin \& Review, 12, 969-992.

Späth, M., Aichert, I., Ceballos-Baumann, A. O., Wagner-Sonntag, E., Miller, N., \& Ziegler, W. (2016). Entraining with another person's speech rhythm: Evidence from healthy speakers and individuals with Parkinson's disease. Clinical Linguistics and Phonetics, 30, 1-18.

Thaut, M. H., Mcintosh, K. W., McIntosh, G. C. \& Hömberg, V. (2001). Auditory rhythmicity enhances movement and speech motor control in patients with Parkinson's disease. Functional Neurology, 16, 163-172.

Wambaugh, J. L. \& Martinez, A. L. (2000). Effects of rate and rhythm control treatment on consonant production accuracy in apraxia of speech. Aphasiology, 14, 851-871. 


\title{
Impairment of switching attention in patients with fluent aphasia and temporal lobe damage
}

\author{
Svetlana Kuptsova ${ }^{1,2}$, Anastasia Ulicheva ${ }^{3}$, Olga Dragoy ${ }^{2}$, Maria Ivanova $^{2,4}$ \\ ${ }^{1}$ Center for speech pathology and neurorehabilitation (Moscow, Russia) \\ ${ }^{2}$ National Research University Higher School of Economics (Moscow, Russia) \\ ${ }^{3}$ Royal Holloway, University of London (United Kingdom) \\ ${ }^{4}$ University of California Berkeley (Berkeley, CA, USA)
}

\section{Introduction}

Previous studies have shown different types of deficits in cognitive and executive functions in patients with aphasia, with attention being one of them (Heuer \& Hallowell, 2015; Murray, 2000,2012 ). Some of these studies have shown impairments in switching between two different tasks in patients with aphasia compared to aged matched healthy controls (Murray et al., 1998; Hula \& McNeil, 2008). But currently there is insufficient information about characteristics of switching attention within one task in patients with aphasia, and whether attention deficits vary depending on type of aphasia and brain lesion site. The aim of this study was to investigate characteristics of switching attention in one type of task in patients with fluent aphasia and temporal lobe damage.

\section{Methods}

\section{Participants}

Twenty healthy individuals (mean age: $50.8 \pm 5.9 ; 10$ men) and sixteen patients with fluent aphasia and temporal lobe damage verified on an MRI scan (mean age: $54.5 \pm 7.9$; 11 men; after left hemisphere CVA, with additional minor damage to the parietal lobe (11 individuals), the occipital lobe (3); ranging from mild to moderate severity of aphasia) participated in this study. All the participants were right-handed native speakers of Russian.

\section{Experimental task}

The participants had to switch their attention between counting high-pitched $(2000 \mathrm{~Hz})$ and low-pitched $(500 \mathrm{~Hz})$ tones that lasted $500 \mathrm{~ms}$ and were presented to them one by one in pseudorandom order. To switch to the next tone the participant had to press the space bar. Each sequence consisted of 7 to 9 tones. There were thirty sequences (trails), half of them had high switching frequency (four switches within a sequence), half of them had low switching frequency (two switches). The participants had to listen to these tones and calculate them separately. At the end of each sequence the participant had to say how many high- and low-pitched tones they had heard. This task had to be done as quickly and accurately as possible. The task was presented on a laptop in an automated mode using the E-Prime 2.0 computer program. The data analysis was performed in SPSS 22.

\section{Results}

Significant differences between healthy controls and patients with aphasia were observed in the number of correct responses for all the trails $(\mathrm{F}=11.65, p=0.002, \mathrm{M}$ (healthy $)=26.65$, $\mathrm{SD}=2.03 ; \mathrm{M}$ (patients $)=23.13, \mathrm{SD}=4.17)$, the number of correct responses for low frequency trials $(\mathrm{F}=9.69, p=0.004, \mathrm{M}$ (healthy $)=13.75, \mathrm{SD}=1.11 ; \mathrm{M}$ (patients $)=11.63, \mathrm{SD}=2.27)$ and reaction times for non-switching stimuli for trials with correct responses $(\mathrm{F}=4.26, p=0.047$, $\mathrm{M}($ healthy $)=1468.90, \mathrm{SD}=391.99 ; \mathrm{M}($ patients $)=1733.93, \mathrm{SD}=612.86)$. No significant differences between the two groups were observed in the number of correct responses for high frequency trials $(\mathrm{F}=2.29, p=0.139, \mathrm{M}$ (healthy $)=12.90, \mathrm{SD}=1.88 ; \mathrm{M}$ (patients $)=11.19, \mathrm{SD}=2.83)$, reaction times across all the stimuli for correct trails $(\mathrm{F}=3.45, p=0.072, \mathrm{M}$ (healthy $)=1600.97$, 
$\mathrm{SD}=427.05 ; \mathrm{M}$ (patients $)=1877.85, \mathrm{SD}=663.41)$ and reaction times for switching stimuli for correct trails $(\mathrm{F}=2.42, p=0.128, \mathrm{M}($ healthy $)=1779.29, \mathrm{SD}=496.19 ; \mathrm{M}$ (patients $)=2063.47, \mathrm{SD}=762.65)$.

\section{Discussion}

Comparison of the number of correct responses in patients with fluent aphasia and temporal lobe damage with the control group revealed that the patients made significantly more errors than the healthy control group. This can be explained by the specificity of the deficits which is typical for patients with fluent aphasia. Patients with fluent aphasia have problems with language comprehension and make a lot of verbal paraphasias in their speech. During the task they might have made verbal paraphasias while providing spoken responses and did not correct these errors. But there are no differences between two groups for trails with high frequency. These trails can be difficult for healthy individuals since they have to switch very fast between stimuli and because of this healthy individuals made a lot of errors. The patients with fluent aphasia made a lot of errors as well (numerically more than the control group), but the difference between the two groups did not reach significance.

Also, patients showed longer reaction times than the control group. However, this effect was observed only for non-switching stimuli in patients, the difference in reaction times between the two groups for the switching stimuli almost reached significance. There was great variability within the patient group in the numbers of correct responses and reaction times. Some patients performed similarly to healthy controls and some substantially worse. This might be due to some kind of lesion differences or severity of aphasia between patients and maybe because of this we did not find any differences between two groups in the high frequency trials and switching stimuli.

A possible explanation for this pattern of results is that patients with fluent aphasia do not seem to evaluate task demands properly and do not deploy substantial amount of additional resources for the switching stimuli. Thus, they are not taking longer to update the count during the switch. Alternatively, it may be the case that patients with temporal damage have impaired sustained attention (required for performance on the non-switching trials) relative to attention switching (required for performance on the switching trials), that is thought to be more dependent on the frontal regions that are intact in this group. Consequently, they are failing more in low frequency sequences, on which correct performance is dependent more on sustained attention. These explanations require further investigation. 


\section{References}

Heuer, S., \& Hallowell, B. (2015). A novel eye-tracking method to assess attention allocation in individuals with and without aphasia using a dual-task paradigm. Journal of Communication Disorders, 55, 15-30

Murray, L. L. (2012). Attention and other cognitive deficits in aphasia: Presence and relation to language and communication measures. American Journal of Speech-Language Pathology, 21:2, 51-64, DOI: 10.1044/1058-0360(2012/11-0067)

Murray, L. L. (2000). The Effects of Varying Attentional Demands on the Word Retrieval Skills of Adults with Aphasia, Right Hemisphere Brain Damage, or No Brain Damage. Brain and Language, 72:1, 40-72

Murray, L. L., Holland, A. L., \& Beeson, P. M. (1998). Spoken language of individuals with mild fluent aphasia under focused and divided-attention conditions. Journal of Speech, Language, and Hearing Research, 41:1, 213-227

Hula, W.D., McNeil, M.R. (2008). Models of attention and dual-task performance as explanatory constructs in aphasia. Seminars in speech and language, 29:3, 169-187 


\title{
Age-related differences in auditory semantic priming: the development of normative electrophysiological data in the Dutch population
}

\author{
Elissa-Marie Cocquyt ${ }^{1}$, Patrick Santens ${ }^{2}$, Pieter van Mierlo ${ }^{3}$, Wouter Duyck ${ }^{4}$, Arnaud \\ Szmalec $^{4,5} \&$ Miet De Letter ${ }^{1}$ \\ ${ }^{1}$ Department of Rehabilitation Sciences, Ghent University, Belgium \\ ${ }^{2}$ Department of Neurology, Ghent University Hospital, Belgium \\ ${ }^{3}$ Department of Electronics and Information Systems, Ghent University, Belgium \\ ${ }^{4}$ Department of Experimental Psychology, Ghent University, Belgium \\ ${ }^{5}$ Psychological Sciences Research Institute, Université catholique de Louvain, Belgium
}

\section{Introduction}

Normal aging is generally associated with a slight decline in executive, phonological and grammatical processing (Glisky, 2007; Stamatakis et al., 2011; Moscoso Del Prado Martin, 2017). Conversely, most aspects of semantic processing are thought to remain fairly stable with age. This maintenance of semantic abilities has been associated with changes in both brain structure (Kemmotsu et al., 2012) and functional networks (Hoffman, 2018). However, heterogeneous aging-effects in semantic processing have been reported by means of the event-related potential (ERP) technique, especially regarding the semantic N400 in the auditory modality (Giaquinto et al., 2007; Räling et al., 2016). Therefore, the aim of this study is to delineate the effects of normal aging on the N400 amplitude and latency. The investigation of semantic potentials in different ages will yield normative electrophysiological data for verbal-semantic comprehension in the Dutch population.

\section{Methods}

One hundred and twenty healthy, right-handed adults participated in this experiment. Ten males and ten females in each of six age-decades, between 20 and 80 years, were evaluated during EEG-recording. The ERP-paradigm was a semantic priming task in which an explicit judgment had to be made on the semantic relatedness of 120 auditorily presented prime-target pairs (adapted from Hagoort, Brown and Swaab, 1996). Both target words in the related and unrelated condition as well as the difference waves were analyzed. Mean amplitudes and onset latencies of the N400-effect were extracted using BrainVision Analyzer 2.1. Aging effects were investigated by means of a linear mixed model.

\section{Results}

All subjects, regardless of age, produced a reliable N400-effect. More precisely, larger N400 amplitudes were elicited by semantically unrelated target words than by semantically related words. With age, the N400-effect showed a decrease in amplitudes and an increase in latencies. Normative data for each of the six age decades were obtained.

\section{Discussion}

This study provides an examination of the age-related changes in amplitudes and latencies of the auditory N400 during a semantic priming task. A reliable N400-effect could be observed throughout the lifespan from 20 to 80 years. Specific aging effects are discussed in the context of neuroscientific theories of aging. Moreover, the normative electrophysiological data for semantic processing will bring added value in the diagnostic stage and the long-term follow-up of aphasic patients of different ages. 


\section{References}

Giaquinto, S., Ranghi, F., \& Butler, S. (2007). Stability of word comprehension with age. An electrophysiological study. Mechanisms of ageing and development, 128(11-12), 628-636. doi:10.1016/j.mad.2007.09.003.

Glisky, E. L. (2007). Changes in Cognitive Function in Human Aging. In D. R. Riddle (Ed.), Brain Aging: Models, Methods, and Mechanisms. Boca Raton (FL): CRC Press/Taylor \& Francis.

Hagoort, P., Brown, C. M., \& Swaab, T. Y. (1996). Lexical-semantic event-related potential effects in patients with left hemisphere lesions and aphasia, and patients with right hemisphere lesions without aphasia. Brain : a journal of neurology, 119 ( Pt 2), 627-649.

Hoffman, P., \& Morcom, A. M. (2018). Age-related changes in the neural networks supporting semantic cognition: A meta-analysis of 47 functional neuroimaging studies. Neuroscience and biobehavioral reviews, 84, 134-150. doi:10.1016/j.neubiorev.2017.11.010.

Kemmotsu, N., Girard, H. M., Kucukboyaci, N. E., McEvoy, L. K., Hagler, D. J., Jr., Dale, A. M., . . . McDonald, C. R. (2012). Age-related changes in the neurophysiology of language in adults: relationship to regional cortical thinning and white matter microstructure. The Journal of neuroscience: the official journal of the Society for Neuroscience, 32(35), 12204-12213. doi:10.1523/jneurosci.0136-12.2012.

Moscoso Del Prado Martin, F. (2017). Vocabulary, Grammar, Sex, and Aging. Cognitive science, 41(4), 950-975. doi:10.1111/cogs.12367.

Räling, R., Schroder, A., \& Wartenburger, I. (2016). The origins of age of acquisition and typicality effects: Semantic processing in aphasia and the ageing brain. Neuropsychologia, 86, 80-92. doi:10.1016/j.neuropsychologia.2016.04.019.

Stamatakis, E. A., Shafto, M. A., Williams, G., Tam, P., \& Tyler, L. K. (2011). White matter changes and word finding failures with increasing age. PloS one, 6(1), e14496. doi:10.1371/journal.pone.0014496. 
Oral Presentations II 


\title{
'A moderate global aphasia': the pattern of language deficits in acute post-surgical tumor patients
}

\author{
Olga Dragoy ${ }^{1,2}$, Ekaterina Stupina ${ }^{1}$, Andrey Zyryanov ${ }^{1}$, Marina Chernova ${ }^{1}$, Elizaveta \\ Gordeyeva $^{1}$, Natalya Gronskaya ${ }^{1}$, Galina Gunenko ${ }^{3}$, Sergey Chernov ${ }^{3,4}$, Dmitry Kopachev ${ }^{5}$, \\ Igor Medyanik ${ }^{6}$, Nikita Pedyash ${ }^{4}$, Igor Pronin ${ }^{5}$, Andrey Sitnikov ${ }^{7}$, Konstantin Yashin ${ }^{6}$, \\ Andrey Zuev ${ }^{4}$ \\ ${ }^{1}$ National Research University Higher School of Economics, Russia \\ ${ }^{2}$ Center for Cerebrovascular Pathology and Stroke, Russia \\ ${ }^{3}$ Federal Center for Neurosurgery (Novosibirsk), Russia \\ ${ }^{4}$ National Medical and Surgical Center named after N.I. Pirogov, Russia \\ ${ }^{5}$ N.N. Burdenko National Scientific and Practical Center for Neurosurgery, Russia \\ ${ }^{6}$ Privolzhsky Research Medical University, Russia \\ ${ }^{7}$ Federal Centre of Treatment and Rehabilitation of the Ministry of Healthcare of the Russian \\ Federation, Russia
}

\section{Introduction}

Prior to a neurosurgical resection, most patients with tumors in the left hemisphere of the brain show normal or close to normal language abilities. After the surgery, however, many patients present with significant language decline, which often resolves over several months (Duffau et al. 2003). Previous studies reported different rates of aphasia in the acute post-operative phase, ranging from $17 \%$ to $100 \%$ (Papagno et al. 2012). Critically, the mechanisms of postsurgical language deficits are not understood, the main question being if they follow a traditional syndromatic pattern of a common post-stroke aphasia. Most studies that attempted to analyze postsurgical language outcomes used incomprehensive language testing (Davies et al. 2005); in others, subjective scoring of tests was used (Wilson et al. 2015). We used the comprehensive and objectively rated Russian Aphasia Test (Ivanova et al. 2016) to examine the pattern of language deficits in the acute phase in patients who underwent a tumor resection in the left hemisphere and compared it to performance of individuals with post-stroke aphasia on the same test.

\section{Methods}

A total of 58 monolingual native Russian-speaking patients (27 female, 31 male; age range 18-63, mean 40 y.o.) with primary brain tumors (WHO grade I-IV) in left-hemisphere perisylvian language regions participated in this study. Three patients were ambidextrous, five were left-handed, while all others were right-handed. All patients were operated for the first time. Forty-one of them underwent awake craniotomy and intraoperative language mapping to minimize postoperative language deficits; seventeen patients were operated under general anesthesia due to contraindications for awake craniotomies.

All patients were assessed with the Russian Aphasia Test (RAT; Ivanova et al. 2016), before and 1-14 days after the surgery. This battery consists of a series of tasks designed to assess both comprehension and production at all levels of linguistic processing. In this study, we focused on three basic levels - phonological, lexical and syntactic, in two modalities -comprehension and production. In comprehension, these were assessed using phonological judgment of minimal pairs of pseudowords; word-to-picture matching for objects and actions; sentence-to-picture matching for syntactic constructions of varying complexity. In production, we used repetition of pseudowords; picture naming of objects and actions; sentence construction in response to a picture. Test performance was measured in percentage of correct responses. We then compared pre- and postsurgical test results. Statistical analysis was performed using SPSS (Version 22). 


\section{Results}

Presurgically, most patients performed well on language tests: 50 patients showed normal or close to normal language abilities; four patients showed mild deficit and four patients presented with a stronger language deficit. In contrast, postsurgical testing revealed that only 13 patients $(22 \%)$ maintained or improved their language performance; others showed different degrees of worsening. At the group level, production was affected significantly more than comprehension $(\mathrm{t}(57)=4.41, p=0.000)$ : performance on language production tasks decreased by $17 \%(\mathrm{SD}=27.7 \%)$, while performance on comprehension tasks decreased by $6.6 \%(\mathrm{SD}=17.2 \%)$.

Changes in patients' performance on three chosen levels of linguistic processing (phonological, lexical and syntactic), in comprehension and production, were further compared. For that, difference between post- and presurgical scores on six aforementioned tests was calculated and entered pair-wise comparisons ( $p$-values were corrected for multiple comparisons using the Bonferroni procedure). Significant differences in comprehension tests were found for the lexical $(\mathrm{t}(57)=2.78, p=0.007)$ and the syntactic $(\mathrm{t}(55)=3.37 p=0.001)$ levels of processing. In production, there were significant differences at all considered levels of processing: phonological $(\mathrm{t}(57)=2.77, p=0.008)$, lexical $(\mathrm{t}(57)=3.90, p=0.000)$ and syntactic $(\mathrm{t}(54)=4.35 p=0.000)$. Interestingly, language worsening was more profound for higher level of processing when compared to lower levels. In comprehension, postsurgical scores decreased by $5.9 \%(\mathrm{SD}=16.1 \%)$ on the lexical and by $10.7 \%(\mathrm{SD}=22.8 \%)$ on the syntactic task. In production, postsurgical scores decreased by $10.1 \%(\mathrm{SD}=27.4 \%)$ on the phonological, by $16.1 \%$ ( $\mathrm{SD}=31.1 \%)$ on the lexical, and $21 \%(\mathrm{SD}=33.8 \%)$ on the syntactic task.

Additionally, we calculated pairwise correlations between postsurgical scores of the six tests. All were strong and positive (r ranging from 0.45 to 0.86 ; significant at the 0.000 level meeting a multiple comparison correction). To compare this pattern with typical post-stroke aphasia, we analyzed the same data of 30 randomly chosen stroke survivors from a different project. In this cohort, the correlation results were very different from the post-surgery group: patients with post-stroke aphasia only showed correlations between functionally related linguistic levels -e.g., phonological perception correlated with word comprehension $(\mathrm{r}=0.60, p=0.001)$, word production - with sentence production $(\mathrm{r}=0.77, p=0.001)$.

\section{Discussion}

Though in the long-term perspective language recovery rate after neurosurgeries is high, in the acute post-surgical phase most of the patients demonstrate a decline in language abilities. These deficits do not follow the classical pattern of post-stroke aphasias, where one can identify a specific locus of the deficit. Instead, all tests showed strong pairwise correlations, meaning that there are no dissociations among different language domains. At the same time, worsening was more profound for higher levels of processing than for lower levels. All in all, our results suggest that the observed deficits are rather non-specific in nature and are modulated by the overall linguistic/cognitive load of the task, since higher linguistic levels require more information to integrate. Future studies that would include lesional data and follow-up longitudinal studies are needed, to further inform the nature of post-surgical language deficits. The study was funded by Russian Foundation of Basic Research (project No. 18-012-00829).

\section{References}

Davies, K.G., Risse, G.L., \& Gates, J.R. (2005). Naming ability after tailored left temporal resection with extraoperative language mapping: increased risk of decline with later epilepsy onset age. Epilepsy \&6 Behavior, 7, 273-278. 
Duffau, H., Capelle, L., Denvil, D., Sichez, N., Gatignol, P., Lopes, M., et al. (2003). Functional recovery after surgical resection of low grade gliomas in eloquent brain: hypothesis of brain compensation. Journal of Neurology, Neurosurgery, and Psychiatry, 74, 901907.

Ivanova, M., Dragoy, O., Akinina, J., Soloukhina, O., Iskra, E., Khudyakova, M., \& Akhutina, T. (2016). AutoRAT at your fingertips: Introducing the new Russian Aphasia Test on a tablet. Frontiers in Psychology Conference Abstract: 54th Annual Academy of Aphasia Meeting.

Papagno, C., Casarotti, A., Comi, A., \& Bello, L. (2012). Measuring clinical outcomes in neuro-oncology. A battery to evaluate low-grade gliomas (LGG). Journal of NeuroOncology, 108(2), 269-275.

Wilson, S.M., Lam, D., Babiak, M., Perry, D., Shih, T., Hess, C.P., ... \& Chang, E.F. (2015). Transient aphasias after left hemisphere resective surgery. Journal of Neurosurgery, 123(3), 581-593 


\title{
Right-hemisphere density reduction in acute post-stroke aphasia
}

\author{
Karine Marcotte ${ }^{1,2}$, Alberto Osa ${ }^{1,2}$, Johémie Boucher ${ }^{3,4}$, Bérengère Houzé ${ }^{3}$, Christophe \\ Bedetti $^{3}$, Amélie Brisebois ${ }^{1}$, Alex Desautels ${ }^{1,5,6}$, and Simona Maria Brambati ${ }^{3,4}$ \\ ${ }^{1}$ Centre de recherche du Centre intégré universitaire de santé et de services sociaux du \\ Nord-de-l'Tlle-de-Montréal, Hôpital du Sacré-Cour de Montréal, Montréal, Québec, Canada. \\ ${ }^{2}$ École d'orthophonie et d'audiologie, Université de Montréal, Montréal, Québec, Canada. \\ ${ }^{3}$ Centre de recherche de l'Institut Universitaire de Gériatrie de Montréal, Montréal, Québec, \\ Canada. \\ ${ }^{4}$ Département de psychologie, Université de Montréal, Montréal, Québec, Canada. \\ ${ }^{5}$ Département de neurosciences, Université de Montréal, Montréal, Québec, Canada. \\ ${ }^{6}$ Centre d'études Avancées en médecine du sommeil, Hôpital du Sacré-Cour de Montréal, \\ Montréal, Québec, Canada.
}

\section{Introduction}

Post-stroke aphasia (PSA) is generally caused by an ischemic stroke in the left middle cerebral artery (MCA). The occlusion of the left MCA causes an interruption of cerebral blood flow, leading to a hypoperfusion of the supplied regions supplied and, eventually, to focal cell death. The territory supplied by the MCA includes a great portion of the frontal, parietal and temporal lobes, which together play a crucial role in the brain language network. Studies investigating the impact of stroke on language functions in PSA have mainly focused in the chronic stage of the disease and on the lesion site within the left hemisphere. One of the few studies investigating the functional changes of acute PSA on the language network showed that PSA within the first 3 days post-stroke do not activate the regions of the language networks neither in the left or the right hemisphere.(Saur et al., 2006) Moreover, secondary neurodegeneration in functionally and structurally connected brain areas, including areas in the contralateral hemisphere, can be observed after an ischemic stroke.(Blum et al., 2012; Schaapsmeerders et al., 2015; Wu et al., 2008) To date, both atrophy and expansion have reported over the long-time course of PSA recovery. However, little is known regarding the gray matter (GM) density of the right hemisphere during the early recovery of PSA. The objective of the present study is to assess whether patients with acute PSA caused by an ischemic stroke in the left MCA present reduced GM density in the right hemisphere compared to healthy controls three days after stroke.

\section{Methods}

\section{Participants}

Twenty-four right-handed PSA (9 women; mean age $=70.9 \pm 12.8$ years) and twenty biological sex- and age-matched neurologically healthy controls (9 women; mean age: $65.0 \pm 7.4$ years; mean education: $11.6 \pm 3.9$ years) were enrolled in the study. Inclusion criteria for PSA were: 1) left-hemispheric stroke in the MCA territory occurred within the previous 72 hours, 2) presenting oral language impairments due to the stroke. Exclusion criteria included: presenting bihemispheric infarcts; previous head injury, stroke or intracranial surgery; history of major psychiatric illness, alcohol or drug abuse; learning difficulties before the stroke; an uncorrected hearing or visual disturbance; contraindication to magnetic resonance.

\section{MRI acquisition parameters}

MRI images (within 3 days post-stroke) were acquired using a Skyra 3T MRI scanner (Siemens Healthcare, USA) at the radiology department of Hôpital du Sacré-Coeur of Montreal. High resolution 3D T1-weighted $(\mathrm{T} 1 \mathrm{w})$ image $(\mathrm{TR}=2200 \mathrm{~ms}, \mathrm{TE}=2.96 \mathrm{~ms}, \mathrm{TI}=900 \mathrm{~ms}, \mathrm{FOV}=$ $250 \mathrm{~mm}$, voxel size $=1 \times 1 \times 1 \mathrm{~mm}^{3}$, matrix $=256 \times 256,192$ slices, flip-angle $=8^{\circ}$ ) was acquired using a Magnetization Prepared Rapid Gradient Echo (MP-RAGE) sequence. A diffusion 
weighted imaging (DWI) sequence was also acquired $(\mathrm{TR}=8051 \mathrm{~ms}$, TE $=86 \mathrm{~ms}, \mathrm{FOV}=230$ $\mathrm{mm}$, voxel size $=2 \times 2 \times 2 \mathrm{~mm}^{3}$, flip angle $=90^{\circ}$, bandwidth $=1698 ;$ EPI factor $=67 ; 68$ slices in transverse orientation) with one image $\left(b=0 \mathrm{~s} / \mathrm{mm}^{2}\right)$ and 64 images with non-collinear diffusion gradients $\left(b=1,000 \mathrm{~s} / \mathrm{mm}^{2}\right)$ in a posterior-anterior $(\mathrm{PA})$ phase encoding direction, as well as two other images $\left(\mathrm{b}=0 \mathrm{~s} / \mathrm{mm}^{2}\right)$ : one in a PA phase encoding direction and the other in an anterior-posterior (AP) phase encoding direction.

\section{Lesion Mapping}

The lesion delineation was performed by a semi-automatic method and verified by a fully manual method. First, Clusterize SPM's toolbox was used to semi-automatically delineate the lesion in the DWI-derived mean diffusivity map for each patient. This SPM toolbox previously showed to have a good reliability in acute lesions delineation in post-stroke patients. Mean diffusivity maps derived from diffusion MRI were suggested to be a promising tool in lesion visualisation in post-stroke acute phase. Clusterize automatically computed hypo-intensities clusters of voxels on mean diffusivity maps. Then, cluster(s) corresponding to the lesion were manually selected and adjusted to fit the lesion in each slice by a study team member. The entire lesion was then extracted for each subject. Secondly, each lesion file was counter-verified and adjusted (if needed) with MI-brain software (Imeka Solutions Inc.), with the help of mean diffusivity and b0 DWI maps by an experienced team member in lesion delineation. Both raters were blind to language/fluency/behavioral scores.

\section{Voxel-based morphometry}

VBM pre-processing was performed using Clinical Toolbox Version 7/7/2016 running on SPM12. The template for normalization was obtained from 30 healthy subjects (mean age: 61.3 years, 17 men) (Rorden, Bonilha, Fridriksson, Bender, \& Karnath, 2012). Eniantiomorphic normalisation method was used to warp images to the template. Lesion maps were entered in the normalization step. The pre-processing of the control group followed the same procedures of PSA patients (without including the lesion). The GM tissue images obtained from the segmentation of normalized images were then smoothed with a FWHM 6 mm Gaussian filter. A two-sample T-test model was used to compared GM density at voxel level between controls and PSA patients. Age, sex and years of education were entered in the model as covariates. Results were assessed using a threshold of significance of $p<0.05$ family-wise error (FWE) corrected at cluster level.

\section{Results}

The VBM results generally revealed that the acute PSA present GM density reduction in the right homologues of the brain regions included in the language network. These results raised the question whether the mainregions showing reduced density are usually coactivated with the left-lateralized language network subserved by the left MCA in healthy subjects. To address this issue we derived meta-analytic maps of co-activation based on NeuroSynth database $(p<0.05$ FDR-corrected) for the most significant coordinates of the VBM analysis. The results confirmed that the regions showing decreased GM density in acute PSA are usually coactivated with the left-lateralized language network in healthy participants.

\section{Discussion}

These results provide evidence of a possible early impact of stroke on the homologue regions of the language network in the right hemisphere in PSA. In healthy controls, these regions are usually coactivated with the left language network subserved by the left MCA and damaged in our PSA samples. These results could provide evidence of possible early structural changes in terms of decreased GM density. 
Table 1. Brain regions showing GM reduction in acute PSA (within three days from stroke) versus controls

\begin{tabular}{lll}
\hline Brain Region & $\mathrm{x}, \mathrm{y}, \mathrm{z}$ & $\mathrm{T}$ score \\
\hline R anterior Middle Temporal Gyrus & $60-17$ & 5.22 \\
& $58-2-20$ & 5.08 \\
& $53-4-34$ & 4.34 \\
& $67-19-18$ & 3.84 \\
R Temporal Pole & $5213-21$ & 4.34 \\
& $5510-9$ & 3.58 \\
R Superior Temporal Gyrus & $47-38$ & 3.55 \\
R Posterior Inferior Temporal Gyrus & $59-6$ & 3.81 \\
R Posterior Middle Temporal Gyrus & $57-65-20$ & 4.16 \\
R Anterior Insula & $69-52-15$ & 3.58 \\
R Supramarginal Gyrus & $67-44-15$ & 3.70 \\
R PostCentral Gyrus & $3517-17$ & 4.61 \\
& $63-2821$ & 5.21 \\
& $59-835$ & 4.50 \\
R Medial superior Frontal Gyrus & $48-2056$ & 4.33 \\
& $52-1248$ & 4.17 \\
R Inferior Frontal Gyrus, pars opercularis & $55-1540$ & 3.37 \\
R Inferior Frontal Gyrus, pars triangularis & 3509 & 5.09 \\
& 54922 & 4.31 \\
R Frontal Operculum & $4544-2$ & 3.70 \\
R Posterior Orbital Gyrus & 45487 & 4.83 \\
R Middle Frontal Gyrus & $4416-1$ & 4.25 \\
& $3923-19$ & 4.64 \\
R Inferior Occipital Gyrus & $2934-20$ & 5.15 \\
& 433232 & 4.94 \\
& 482920 & 4.73 \\
& $46-76-17$ & 4.32 \\
& $57-60-19$ & 4.26 \\
\hline
\end{tabular}

\section{References}

Blum, S., Luchsinger, J. A., Manly, J. J., Schupf, N., Stern, Y., Brown, T. R., ... Brickman, A. M. (2012). Memory after silent stroke: Hippocampus and infarcts both matter. Neurology. https://doi.org/10.1212/WNL.0b013e31823ed0cc

Rorden, C., Bonilha, L., Fridriksson, J., Bender, B., \& Karnath, H. O. (2012). Age-specific CT and MRI templates for spatial normalization. NeuroImage. https://doi.org/10.1016/j.neuroimage.2012.03.020

Saur, D., Lange, R., Baumgaertner, A., Schraknepper, V., Willmes, K., Rijntjes, M., \& Weiller, C. (2006). Dynamics of language reorganization after stroke. Brain: A Journal of Neurology, 129(Pt 6), 1371-84. https://doi.org/10.1093/brain/awl090

Schaapsmeerders, P., van Uden, I. W. M., Tuladhar, A. M., Maaijwee, N. A. M., van Dijk, E. J., Rutten-Jacobs, L. C. A., ... Kessels, R. P. C. (2015). Ipsilateral hippocampal atrophy is associated with long-term memory dysfunction after ischemic stroke in young adults. Human Brain Mapping. https://doi.org/10.1002/hbm.22782

Wu, W., Brickman, A. M., Luchsinger, J., Ferrazzano, P., Pichiule, P., Yoshita, M., ... Small, S. A. (2008). The brain in the age of old: The hippocampal formation is targeted differentially by diseases of late life. Annals of Neurology. https://doi.org/10.1002/ana.21557 


\title{
Validation of the Rapid Aphasia Test in the Russian-speaking post-stroke population
}

\author{
Olga Buivolova ${ }^{1}$, Oxana Vinter $^{2}$, Roelien Bastiaanse ${ }^{1,3}$, Olga Dragoy ${ }^{1,4}$ \\ ${ }^{1}$ National Research University Higher School of Economics \\ ${ }^{2}$ Moscow Municipal Clinical Hospital N31 \\ ${ }^{3}$ Center for Language and Cognition Groningen (CLCG), University of Groningen \\ ${ }^{4}$ Center for Cerebrovascular Pathology and Stroke
}

\section{Introduction}

The Aphasia Rapid Test (ART; Azuar et al., 2013) is a 3-minute bedside screening tool allowing each member of medical staff to assess speech and language abilities in individuals in the acute stroke period. This test has been developed as a 26-point scale rating language production and comprehension as well as the presence of articulation disorders. The ART is standardized, normalized and adapted for different languages. In Russian acute stroke clinics, there is, so far, no standardized speech and language screening test. Instead, clients are diagnosed with the long and elaborate Vasserman's scale (Vasserman, Dorofeeva, \& Meerson, 1997), assessing not only speech and language but also other cognitive and motor functions. This test should be administered by professional speech and language therapists or neuropsychologists who are often not available in clinics for acute stroke patients. The goal of the current study was to adapt the Aphasia Rapid Test for Russian and to validate it for aphasic individuals in chronic and acute clinical populations. Furthermore, the potential of the Russian ART for assessment of speech and language recovery dynamics has been evaluated.

\section{Methods}

\section{Materials and Design}

The materials of the test have been adapted from the English version of ART (Azuar et al., 2013) using original stimuli relevant for Russian. The first task assesses a patient's ability to follow two simple and two complex instructions (to open and close the eyes; to give the left hand; to put the left hand on the right ear). In the second task, participants are asked to repeat three single nouns with different numbers of articulatory switches (kit 'whale' - no switch, groza 'thunderstorm' - 1 switch, vorotnik 'collar' - 3 switches). The third task assesses repetition of one simple sentence (Mama kupila dva zelehykh yabloka 'Mom bought two green apples'). The fourth task is the naming of three objects presented in the pictures (myach 'ball', zvezda 'star', kompas 'compass'). Finally, in a 1-minute semantic fluency task participants are asked to name as many animals as possible. Dysarthria severity is also assessed. All tasks are scored in the same manner as in the original ART (Azuar et al., 2013), that is, 0 means absence of dysarthria and 3 points correspond to very severe disorder. For validation of the ART, the iPad version of the Token Test, the "golden standard" for diagnosing aphasia and estimating its severity, was used (Token Test App: Bastiaanse, Raaijmakers, Satoer, \& Visch-Brink, 2016; Russian version: Akinina et al., 2016).

\section{Participants and Procedure}

The Russian ART was tested for its validity in the clinical group of 16 people with chronic aphasia (9 females; mean age $=54(\mathrm{SD}=8.9)$; range $41-71$; mean time post onset $=23.7$ months ( $\mathrm{SD}=27.1)$; range 2-84 months). Fourteen participants from this group performed the Russian ART and the Token Test twice for establishing the test-retest reliability in people with stable linguistic status. To evaluate whether the Russian ART reflects speech and language problems in acute stroke period, we tested 8 individuals ( 3 females; mean age $=62.5$ ( $\mathrm{SD}=$ $9.6)$; range $49-87$; mean time post onset $=6.3$ days $(\mathrm{SD}=3.4)$; range $3-12$ days $)$ with the ART 
and the Token Test. Additionally, 16 people (9 females; mean age $=71.4(\mathrm{SD}=13.7)$; range 40-85; mean time post onset $=7.6$ days $(\mathrm{SD}=3.7)$; range 3 - 14 days $)$ in the acute stroke period were recruited to measure if the Russian ART is suitable for estimating the recovery dynamics. This group was tested twice with the Russian ART at two time points with an average of 4 days between them.

\section{Results}

In the chronic clinical group, the mean overall ART score was 6 ( $\mathrm{SD}=4.2$, range $1-15$, $\max .=$ 26) and the mean overall Token Test score was $19.9(\mathrm{SD}=8.1$, range $6-30$, max. $=36)$. There was a significant negative association between the performance on the ART and the Token Test (Pearson's $\mathrm{r}=-.649, p<.001)$. In the clinical group of people in acute stroke period, the mean overall ART score was $7(\mathrm{SD}=5.6$, range $1-8)$ and the mean overall Token Test score was 24.6 ( $\mathrm{SD}=10.7$, range $0-34)$. Three participants in this group were considered as non-aphasic by the Token Test, however, dysarthria was detected with the Russian ART. In this group, there was again a significant correlation between the Russian ART and the Token Test $(\mathrm{r}=-.968, p$ $<.001)$. The revealed associations were negative because more severe aphasia corresponds to higher scores on the ART but lower scores on the Token Test. The results of Student's t-test showed no significant difference between the performance of individuals with chronic aphasia on the Russian ART at the first and the second testing time points ( $\mathrm{t}(13)=1.449, p=.171$ ) meaning high test-retest reliability. In the acute clinical group, the difference between two time points was significant $(\mathrm{t}(15)=3.280, p=.005)$, meaning that the test reflects the speech and language recovery in the acute post-stroke period. The control group performed at ceiling on the ART: all participants scored 0 points. The mean Token Test score was 32.7 (SD = 1.8, range $29.5-35)$.

\section{Discussion}

Our results showed that Russian ART meets the standards for clinical tests and can be considered as a valid tool for use in clinical settings, both in the chronic and in the acute stage. The high correlation between scores obtained on the Russian ART and the "golden standard" Token Test proved that this test reliably detects speech and language problems and their severity. The stability of the test scores at two time points in a chronic clinical group and significant changes in the acute clinical group showed that Russian ART reflects the dynamics of recovery in the first days post onset. Therefore, Russian ART could be suggested for usage in a Russian speaking acute clinical population.

\section{References}

Akinina, Yu., Dragoy, O., Raaijmakers, S., Satoer, D. \& Bastiaanse, R. (2015). The e-Token Test: Russian version. Groningen (NL): Groningen Expert Center for Language and Communication Disorders.

Azuar, C., Leger, A., Arbizu, C., \& Samson, Y. (2013). The Aphasia Rapid Test: An NIHSSlike aphasia test, Journal of Neurology, 2110-2117. https://doi.org/10.1007/s00415-0136943-x

Bastiaanse, R., Raaijmakers, S., Satoer, D., \& Visch-Brink, E. (2016). The Multilingual Token Test. Aphasiology, 30(4), 508-508. https://doi.org/10.1080/02687038.2015.1121710.

Vasserman, L. I., Dorofeeva S. A., Meerson Ya. A. (1997) Metody neiropsikhologicheskoy diagnostiki: Prakticheskoe rukovodstvo. 


\title{
Psychometric Properties of the Token Test App
}

\author{
Yulia Akinina ${ }^{1,2}$, Olga Buivolova ${ }^{1}$, Olga Soloukhina ${ }^{1}$, Roelien Bastiaanse ${ }^{1,2}$ \\ ${ }^{1}$ National Research University Higher School of Economics, Moscow, Russia \\ ${ }^{2}$ University of Groningen, Groningen, the Netherlands
}

\section{Introduction}

Token Test (TT, De Renzi \& Vignolo, 1962) is an established tool for assessing auditory comprehension in aphasia and other language disorders. Tokens of different shape, size and color are presented to the participant, and they have to manipulate them following auditory instructions of increasing complexity. The test score indicates presence and severity of the deficit. Since the original publication, many variants of the test and versions for different languages have been developed.

Presently, digital editions of diagnostic tools are in high demand due to their advantages over paper-and-pencil versions, such as lower human error, standardization of the procedure, automatic presentation and scoring etc. (Newton, Acres, \& Bruce, 2013). This motivated the development of a tablet application based on the 36-item version of the TT (de Renzi \& Faglioni, 1978). Other rationales were that a tablet app is more readily available than a test that requires physical tokens, and that new language versions can be implemented relatively easily. Thus, the electronic version of the TT (eTT; Bastiaanse, Raaijmakers, \& Satoer, 2015) for iOS was created. A pilot project with Russian-speaking neurologically healthy individuals (NHI) and people with aphasia (PWA) demonstrated promising psychometric properties: good sensitivity, test-retest reliability, and concurrent validity both with the paper-and-pencil TT and standard language examination (Akinina et al., 2017).

In the current version of the app (Bastiaanse et al., 2018), several critical bugs were corrected, and an Android version was added. The new edition contains around 40 languages (Afrikaans, Akan, Albanian, Armenian, Berber, Bosnian, Catalan, Catalan form Valencia, Chinese Mandarin from Mainland and Taiwan, Croatian, Czech, Danish, Dutch, English (American, Australian, British, Canadian, and South African), Finnish, Flemish, French, Frisian, Galician, German, Greek, Hebrew, Hungarian, Maltese, Norwegian, Persian, Portuguese, Brazilian Portuguese, Russian, Spanish, Swiss German, Tagalog, Tatar, and Turkish). The development of several other language versions is in progress.

Presently, the standard psychometric properties (sensitivity and specificity, test-retest reliability, concurrent validity etc.) are being collected in Russian-speaking NHI and PWA. Parts of the data were presented elsewhere (e.g., test-retest reliability and practice effects in NHI: Akinina et al., submitted). Here, we present preliminary data on eTT performance in groups of Russian-speaking NHI and fluent and non-fluent PWA. 


\section{Methods}

\section{Participants}

The NHI group currently consists of 55 right-handed neurologically healthy speakers of Russian. There are 33 females, mean age is 39.75 yo (range $18-75, S D=18.66$ ). The PWA group currently consists of 26 Russian-speaking people with fluent $(\mathrm{N}=8)$ and non-fluent $(\mathrm{N}=18)$ aphasia of various etiology at subacute and chronic stage. There are nine females, mean age is 58.54 yo (range $26-74, S D=13.03$ ).

\section{Procedure}

The tokens are demonstrated on a tablet screen, and the 36 item instructions, as well as general instructions, recorded by a professional speaker, are presented automatically. The instructions are organized in 6 blocks. In blocks 1-5, the instructions are to touch one or two of the tokens; in block 6, the instructions are to do something with the tokens (e.g., "Put the red circle between the yellow square and the green square").

The responses are scored automatically: 1 for a correct response, 0.5 for a correct response after item repetition (allowed in blocks 1-5); 0 for an incorrect response. Hence, the possible score range is $0-36$. The test is discontinued after five incorrect responses in a row; in block 6 , all the items are always presented.

\section{Results}

The descriptive statistics of the eTT total scores for NHI, fluent PWA, non-fluent PWA, and PWA in general are presented in Table 1 . We did not perform any statistical tests yet, because of the preliminary nature of the data.

Table 1. eTT total score

\begin{tabular}{lllllll}
\hline Group & mean & median & SD & IQR & $\min$ & $\max$ \\
\hline NHI $(\mathrm{N}=55)$ & 34.86 & 35 & 1.37 & 1.77 & 30 & 36 \\
PWA, fluent $(\mathrm{N}=8)$ & 15.44 & 12.5 & 12.13 & 12.88 & 1.5 & 34.5 \\
PWA, non-fluent $(\mathrm{N}=18)$ & 18.39 & 18.5 & 10.12 & 13.13 & 1 & 36 \\
PWA, general $(\mathrm{N}=26)$ & 17.48 & 16.5 & 10.62 & 15.63 & 1 & 36 \\
\hline
\end{tabular}

\section{Discussion}

At this stage, with an incomplete dataset, we cannot draw definitive conclusions regarding the psychometric properties of the eTT. However, preliminary data show that, as expected, NHI scores are almost at ceiling, and both PWA groups perform worse and with greater variability. The test performance statistics - sensitivity, specificity, differences between groups of PWA, correlations with standard severity scores -will be calculated and reported when data collection is complete ( $\mathrm{N}=100$ in both NHI and PWA groups). Also, the test-retest scores and concurrent validity of eTT and the paper-and-pencil TT will be collected in separate groups of PWA. Overall, the eTT, together with its psychometrics parameters, will be a useful standardized test for aphasia diagnostics.

\section{References}

Akinina, Y., Soloukhina, O., Bastiaanse, R. (submitted). Automated language deficit diagnostics with the Token Test App: test-retest reliability and practice effects. Abstract submitted to Yth International Conference on Serious Games and Applications for Health, IEEE SeGAH 2019, to be held on Kyoto, Japan, from 5 - 7 of August 2019.

Akinina, Y., Buivolova, O., Iskra, E., Silaeva, V., Soloukhina, O., Bastiaanse, R. (2017). Token Test on a tablet: a case of adaptation. Poster presented at 14th European Conference of Psychological Assessment, July $201 \%$. 
Bastiaanse, R., Akinina, Yu., Satoer, D., Visch-Brink, E.G. (2018). The Token Test App, Second Edition. Groningen (NL): The University of Groningen.

Bastiaanse, R., Raaijmakers, S., Satoer, D. (2015). The e-Token Test. Groningen (NL): Groningen Expert Center for Language and Communication Disorders

De Renzi, E., Faglioni, P. (1978). Normative data and screening power of a shortened version of the Token Test. Cortex, 14, 41-49.

De Renzi, E., Vignolo, L.A. (1962). The Token Test: a sensitive test to detect receptive disturbances in aphasics. Brain, 85, 665-678.

Newton, C., Acres, K., Bruce, C.A. (2013). Comparison of computerized and paper-based language tests with adults with aphasia. American Journal of Speech-Language Pathology, 22, 185-197. 


\title{
Coupling language and executive functions for premanifest and early Huntington's Disease follow up
}

\author{
C. Jacquemot ${ }^{1,2}$, C. Schramm ${ }^{1,2}$, L. Lemoine ${ }^{1-3}$, K. Youssof ${ }^{1-3}$, AC. Bachoud-Lévi ${ }^{1-3}$ \\ ${ }^{1}$ DEC-ENS, PSL University, Paris \\ ${ }^{2}$ Inserm U955-E01, IMRB-UPEC, Créteil \\ ${ }^{3}$ AP-HP, Centre de référence Maladie de Huntington, Service de Neurologie, Hôpital \\ Mondor-Chenevier, Créteil
}

\section{Introduction}

Huntington's disease (HD) is a neurodegenerative disease that primarily affects the striatum and leads to progressive motor, neuropsychiatric and cognitive symptoms. Clinical diagnosis is based on motor symptoms on the Unified Huntington's Disease Rating Scale (UHDRS) and is generally done in the fourth decade of life. There is not curative treatment and death occurs in approximatively 15 years. In the last years, the premanifest stage - the period before clinical diagnosis- emerged as a window of opportunity to reduce the effects of HD by using innovative therapies such as cell grafting or gene therapy that can preserve neural function and thus delay the onset and progression of symptoms[1]. Since HD mutation is autosomal dominant and fully penetrant, it is possible to follow premanifest mutation carriers (preHD) long before their clinical diagnosis. However there is currently no reliable marker sensitive enough to detect infra-clinical signs of disease and monitor their progression in small cohort of subjects[2].

To address this issue and to develop tools for the follow-up of small cohorts of preHD gene carriers and HD patients, we capitalized on the fact that cognitive symptoms, and in particular language and executive disorders, occur before the clinical onset of the disease and are highly sensitive for ascertaining deterioration in $\operatorname{HD}[3-4]$. We designed an intuitive naming task, the CATEX (Category-Exemplar) that assesses executive functions in interaction with language.

First, in a prospective cohort study of preHD, we assessed whether the CATEX score is as an early marker of cognitive signs of HD. We coupled behavioural and Voxel Based Morphometry (VBM) data to explore the correlation between cognitive performance and gray matter volume in preHD. Second, in a 20 month-interval follow-up study of early HD patients, we assessed whether the CATEX score is a more sensitive tool than the UHDRS to monitor disease evolution.

\section{Methods}

CATEX naming task

The participant is asked to produce a single word describing two pictures either of the same item ("Exemplar" condition [N=31], e.g. an open notebook and a closed notebook, with "notebook" as the expected response) or of two different items from the same category ("Category" condition [N=31], e.g. a fork and a knife, with "cutlery" as the expected response). Executive demand differs in the exemplar and category conditions: naming category words makes greater demands on executive resources than naming exemplar words.

\section{Participants}

PreHD $(\mathrm{N}=19)$ and controls $(\mathrm{N}=38)$ were assessed with the CATEX. PreHD were also evaluated with the UHDRS and underwent a MRI scanning.

Early HD patients $(\mathrm{N}=12)$ were assessed with the CATEX and the UHDRS twice within an interval of 20 months.

PreHD: PreHD and controls' CATEX score was compared using an analysis of covariance. 
PreHD were followed-up and cox-proportional hazards regression models for survival data (converters and non-converters) was computed separately for Category and Exemplar scores to relate the time that passes before the clinical diagnosis of HD. The optimal thresholds of CATEX scores to discriminate preHD who became symptomatic (converters) from controls was defined using the receiver operating characteristic (ROC) curve.

A multiple regression analysis explored the correlation between Category and Exemplar score, and gray matter volume in preHD within the ROI of gray matter atrophy.

HD patients: Category and Exemplar scores, and the cognitive and motor scores of the UHDRS at the first and second assessment were compared using Mann-Withney-Wilcoxon test for paired data.

\section{Results}

\section{PreHD}

Controls perform higher than preHD for Category (respectively, 91.2\% SD:2.1 and 79.1, SD: $2.2 ; p=0.001$ ) and Exemplar condition (respectively, 94.5\% SD:2.1, 85.4\%, SD: $2.2 ; p=0.001$ ). During the follow-up period, 9/19 preHD became HD patients. Cox proportional hazard model shows that higher Category score significantly reduces the risk to become symptomatic within the next 4 years (Hazard Ratio $=.81 ; 95 \%$ CI $[0.66,0.98], p=0.031$ ) whereas higher Exemplar score did not $(p=0.63)$. Optimal threshold to discriminate preHD who became symptomatic and controls was estimated to $25.5 / 31$ (95\% CI [24.5, 26.5]) for Category indicating that premanifest gene carriers who perform below this score are close to clinical diagnosis with a Sensitivity of $1.00(95 \%$ CI $[0.78,1])$ and a Specificity of $0.76(95 \%$ CI $[0.66,0.95])$.

The multiple regression analysis between Category score and gray matter volume showed a significant positive correlation in the left anterior putamen $\left(P_{\text {corrected }}<0.05\right)$.

\section{HD patients}

Exemplar score significant declines within the 20-month period (for all, median (Max score=31) and [Q1-Q3] are reported; Exemplar, M0: 29.0 [26.5;30.0], M20: 26.0 [23.8;28.0], V = 53, $p$ $=.010$ ) whereas this is not observed for Category score (Category, M0: 23.0 [20.8;26.3], M20: $24.0[20.3 ; 26.0], \mathrm{V}=35, p=0.9$ ). The UHDRS cognitive scores do not decline (Stroop interference, M0: 28.0 [19.5;33.5], M20: 26 [17.8;29.5], $\mathrm{V}=46, p=0.2$; SDMT, M0: 22.0 [19.0;33.5], M20: $22.5[18.5 ; 30.0], \mathrm{V}=40, p=0.2$; Letter verbal fluency M0: 27.0 [24.5;32.0], M20: 26.0 $[21.8 ; 32.5], \mathrm{V}=38, p=0.31)$. The UHDRS motor score shows a significant decline (M0: $28.5[20.0 ; 34.3], \mathrm{M} 20$ : median $=33.5[25.3 ; 48.0], \mathrm{V}=8, p=0.02)$. When applying correction for multiple comparison, only the Exemplar score remains marginally significant $(p=0.060)$, whereas all the other scores become non-significant (all $p s>.10$ ).

\section{Discussion}

The CATEX is an ecological measure that combines language and executive functions. The Category score is highly sensitive to early cognitive signs of HD and can be used to identify preHD individuals who are within 4 years of clinical diagnosis with a sensitivity of 1 . The Category score is correlated with the striatum volume. In contrast, the Exemplar score is sensitive enough to track disease progression over a period of 20 month in 12 HD patients and constitutes a marker of cognitive decline tailored for the follow-up of small cohort of patients and clinical trials.

\section{References}

1 Tabrizi SJ, Scahill RI, Durr A, Roos RA, Leavitt BR, Jones R, et al. Biological and clinical changes in premanifest and early stage Huntington's disease in the TRACK-HD study: 
The 12-month longitudinal analysis. Lancet Neurol. 2011; 10: 31-42.

2 Tabrizi SJ, Scahill RI, Owen G, Durr A, Leavitt BR, Roos RA, et al. Predictors of phenotypic progression and disease onset in premanifest and early-stage Huntington's disease in the TRACK-HD study: analysis of 36-month observational data. Lancet Neurol. 2013; 12: 637-649.

3 Hinzen W, Rosselló J, Morey C, Camara E, Garcia-Gorro C, Salvador R, et al. A systematic linguistic profile of spontaneous narrative speech in pre-symptomatic and early stage Huntington's disease. Cortex 2018; 100: 71-83.

4 Hart EP, Dumas EM, Giltay EJ, Middelkoop HAM, Roos RAC. Cognition in Huntington's Disease in Manifest, Premanifest and Converting Gene Carriers over Ten Years. J. Huntingtons. Dis. 2013; 2: 137-147. 
Poster Session II 


\title{
The role of the white matter pathways in spontaneous speech in aphasia
}

\author{
Svetlana Averina $^{1}$, Olga Dragoy ${ }^{2,3}$, Roelien Bastiaanse ${ }^{2,4}$ \\ ${ }^{1}$ International Doctorate in Experimental Approaches to Language and Brain (IDEALAB), \\ Universities of Groningen (NL), Newcastle (UK), Potsdam (DE), Trento (IT) and Macquarie \\ University Sydney (AUS) \\ ${ }^{2}$ National Research University Higher School of Economics (RUS) \\ ${ }^{3}$ Center for Cerebrovascular Pathology and Stroke (RUS) \\ ${ }^{4}$ Center for Language and Cognition Groningen, University of Groningen (NL)
}

\section{Introduction}

There is a growing body of evidence linking the speech production characteristics to white matter tracts integrity in chronic aphasia. Speech fluency is often used as a primary behavioural outcome measure and recently it has been linked to damage to the left arcuate fasciculus (Fridriksson et al., 2013, Basilakos et al., 2014, Marchina et al., 2011, Wang et al., 2013), the left aslant tract (Basilakos et al., 2014), and the left uncinate fasciculus (Fridriksson et al., 2013, Hope et al., 2015). The diversity of localizations may be induced by the heterogeneity of the terminology and, hence, to the methods of evaluation for speech fluency. Also, the variety of scales (Fridriksson et al., 2013, Basilakos et al., 2014) and scoring systems (Hope et al., 2015) for speech fluency do not allow to extrapolate the findings over spontaneous speech features in aphasia. In addition, there is no evidence on whether spontaneous speech characteristics in aphasia are related to the same neural correlates as the overall verbal communication limitations.

In the current study, we aim to investigate the role of the white matter tracts in spontaneous speech production and verbal communicative abilities in chronic aphasia. We do not limit our investigation to the ipsilesional white matter, considering the recent findings on the role of the right hemisphere tracts in post-stroke aphasia (Forkel et al., 2014, Forkel \& Catani, 2018).

\section{Methods}

\section{Participants}

Twenty-eight native Russian speakers who suffered from a single left hemisphere stroke resulting in chronic aphasia (from 6 months post-onset) were included in the study. The participants were recruited while they were on the waiting list for admission to the Center for Speech Pathology and Neurorehabilitation (Moscow).

\section{Linguistic assessment}

For every participant, the language performance was assessed two times (T1 - T2) with approximately 4 weeks difference during which they did not receive speech and language therapy. The severity of aphasia was established with the Token Test via App (Akinina et al., 2015). A 200-word spontaneous speech sample was elicited from every participant by an interview with open-ended questions. The interview was audio-recorded and then orthographically transcribed. The transcription was used to quantify speech rate (words per minute), mean length of utterance (MLU) in words, percentage of correct sentences, percentage of sentences with embedded clauses, total number of nouns and lexical verbs (tokens) and number of nouns and verbs types (that were used for the type-token ratios for nouns and verbs). The Amsterdam-Nijmegen Everyday Language Test (ANELT; Blomert et al., 1994; Russian adaptation: Akinina, 2017) was used to examine verbal communicative abilities. 


\section{Neuroimaging}

Diffusion imaging data were obtained at T2. The data were collected using a $1.5 \mathrm{~T}$ Siemens scanner and a sequence specialized for tractography (64 directions, $2.5 \mathrm{~mm}$ isovoxel, $\mathrm{b}=1000$ $\mathrm{s} / \mathrm{mm} 2$, two repetitions with opposite phase encoding directions). After preprocessing in FSL (Jenkinson et al., 2012) and ExploreDTI (http://www.exploredti.com) using the deterministic diffusion tensor imaging approach, all tracts of interest were reconstructed in TrackVis (http://www.trackvis.org) manually. We focused on the long associative tracts in both hemispheres: the three segments of the arcuate fasciculus, the aslant tract, the uncinate fasciculus, the inferior fronto-occipital fasciculus, and the inferior longitudinal fasciculus. The tracts statistics (voxel count, mean length, FA and MD values) were further extracted and used together with behavioral data (as well as demographic data) to build regression models.

\section{Results}

At the current stage, we have collected all the data and finalized the behavioral analysis. The neuroimaging analysis is at its final phase. The results of this ongoing study will indicate whether there are white matter correlates for the spontaneous speech characteristics and the verbal communicative abilities in chronic aphasia.

\section{Discussion}

Our findings will contribute to establishing the link between spontaneous speech and verbal communication performance in chronic aphasia and the integrity of damaged or spared white matter tracts. The results may help to overcome the limitations of the previous studies by using a wider set of spontaneous speech parameters and focusing on both cerebral hemispheres.

\section{References}

Akinina, Yu., Dragoy, O., Raaijmakers, S., Satoer, D. \& Bastiaanse, R. (2015). The e-Token Test: Russian version. Groningen (NL): Groningen Expert Center for Language and Communication Disorders.

Basilakos, A., Fillmore, P. T., Rorden, C., Guo, D., Bonilha, L., \& Fridriksson, J. (2014). Regional white matter damage predicts speech fluency in chronic post-stroke aphasia. Frontiers in Human Neuroscience, 8, 845.

Blomert, L., Kean, M. L., Koster, Ch. \& Schokker, J. (1994). Amsterdam-Nijmegen everyday language test: construction, reliability and validity. Aphasiology, 8, 381-407.

Forkel, S. J., Thiebaut de Schotten, M., Dell'Acqua, F., Kalra, L., Murphy, D. G. M., Williams, S. C. R., \& Catani, M. (2014). Anatomical predictors of aphasia recovery: A tractography study of bilateral perisylvian language networks. Brain, 137, 2027-2039.

Forkel, S.J., Catani, M. (2018). Lesion mapping in acute stroke aphasia and its implications for recovery. Neuropsychologia, 115, 88-100.

Fridriksson, J., Guo, D., Fillmore, P., Holland, A., \& Rorden, C. (2013). Damage to the anterior arcuate fasciculus predicts non-fluent speech production in aphasia. Brain, 136, 3451-3460.

Hope, T. M. H., Seghier, M. L., Prejawa, S., Leff, A. P., Price, C. J. (2015). Distinguishing the effect of lesion load from tract disconnection in the arcuate and uncinate fasciculi. NeuroImage, 125, 1169-1173.

Marchina, S., Zhu, L. L., Norton, A., Zipse, L., Wan, C. Y., \& Schlaug, G. (2011). Impairment of speech production predicted by lesion load of the left arcuate fasciculus. Stroke, 42, $2251-2256$.

Wang, J., Marchina, S., Norton, A. C., Wan, C. Y., \& Schlaug, G. (2013). Predicting speech fluency and naming abilities in aphasic patients. Frontiers in Human Neuroscience, 7 , 831. 


\title{
Musical and linguistic syntactic processing in agrammatic aphasia: An ERP study
}

\author{
Brianne Chiappetta ${ }^{1}$, Matthew Walenski ${ }^{1}$, Elena Barbieri ${ }^{1}$, Aniruddh Patel $^{2}$, Cynthia K. \\ Thompson $^{1}$ \\ ${ }^{1}$ Northwestern University, Department of Communication Sciences and Disorders \\ ${ }^{2}$ Tufts University, Department of Psychology
}

\section{Introduction}

Music, like language, contains discrete elements that are organized within a hierarchical structure, creating a musical syntax. Syntactic processing both in music and in language involves the prediction and integration of discrete elements (e.g., words or chords) into higher-order structures (e.g., sentences or musical phrases). The ability to process syntax in language can be impaired in agrammatic aphasia. However, it is unclear if agrammatic aphasia affects musical syntactic processing as well. The Shared Syntactic Integration Resource Hypothesis (SSIRH; Patel, 2003) suggests that syntactic processing of music and language draws on distinct networks for discrete elements (e.g., separate long-term knowledge stored for words vs. tones), but relies on at least partially shared resources for integrating those elements into the context of a sentence or musical phrase. One key prediction of the SSIRH is that people with a linguistic syntactic impairment, such as those with agrammatic aphasia, should also present with a musical syntactic impairment.

Consistent with the SSIRH, violations of linguistic and musical syntax elicit the same electrophysiological response in healthy adults: the P600 event-related potential (ERP) component (Patel, Gibson, Ratner, Besson, \& Holcomb, 1998). The P600 has been interpreted as an index of integration and revision/reanalysis processes (Kaan, Harris, Gibson, \& Holcomb, 2000; Friederici, Hahne, \& Saddy, 2002). In response to morpho-syntactic violations (subject-verb number disagreement), individuals with agrammatic aphasia present with a P600 with reduced amplitude compared to healthy controls (Wassenaar, Brown, \& Hagoort, 2004).

In the present study, we examined the electrophysiological responses to violations of both linguistic and musical syntax in a group of individuals with stroke-induced chronic agrammatic aphasia and in a group of healthy adults. Based on the SSIRH, we predicted that individuals with agrammatic aphasia would show reduced and/or delayed ERP responses (namely, the P600) to both types of violations, in line with the assumption that musical and linguistic syntactic processing rely on partially shared neural resources.

\section{Methods}

\section{Participants}

To date, four participants with agrammatic aphasia ( 2 female, 2 male; mean age $=45.8$ years, range: $26-66$ ) and five healthy controls ( 2 female, 3 male; mean age $=58.8$ years, range: $44-67$ ) have participated ${ }^{1}$. All participants were right-handed, monolingual, native English speakers with no history of neurological, psychiatric, speech, language, or learning impairments. The participants with aphasia suffered a single left hemisphere stroke at least one year prior to the study, and were diagnosed with agrammatic aphasia following completion of a battery of standard language tests. All participants underwent a full neuropsychological exam including a cognitive screener, a hearing screener, and a pitch discrimination task. They also completed a musical experience questionnaire in order to provide a comprehensive summary of the extent of their musical exposure and experiences (Goldsmiths Musical Sophistication Index; Gold-MSI

\footnotetext{
${ }^{1}$ Data from the full cohort of participants ( $n=20$ per group) is anticipated and will be reported at the meeting.
} 
v1.0; Müllensiefen, Gingras, Musil, \& Stewart, 2014).

\section{Stimuli and Procedure}

All participants completed an auditory grammatical judgment task while EEG was recorded from 32 scalp electrodes. The stimuli were taken from previous studies, and included 100 sentences (Barbieri et al., 2018) and 144 musical phrases (Patel et al., 1998). Half of the sentences were grammatical (e.g., The hiker was camping on the mountain), and half contained a morpho-syntactic violation (e.g., The hikers was camping on the mountain). Half of the musical phrases were grammatical, (all chords were in-key) and half included a target chord that was structurally less expected based on norms in Western Tonal music (contained one chord that was out-of-key). We also included filler sentences and musical phrases (not described here). ERPs were time locked to the onset of the auxiliary verb for sentences and to the onset of the target chord for musical phrases, with a $200 \mathrm{~ms}$ pre-stimulus baseline and $1200 \mathrm{~ms}$ epoch for each.

\section{Results}

Preliminary results indicate that the healthy controls showed a P600 effect in response to violations of morpho-syntax (Figure 1a). The participants with agrammatic aphasia also had a P600 effect in response to violations of morpho-syntax (Figure 1b), although it was delayed relative to the healthy controls. In response to violations of musical syntax, the healthy adults presented with a P600 effect (Figure 1c); the participants with aphasia showed a robust P600 effect (Figure 1d), which was larger in amplitude compared to the healthy controls.

\section{Discussion}

For the morpho-syntactic violations, the P600 effect from the healthy controls is consistent with previous findings (Wassenaar et al., 2004; Barbieri et al., 2018). For individuals with aphasia, the finding of a P600 that was delayed compared to healthy individuals suggests impaired, although not completely suppressed, morpho-syntactic processing in our participants. The finding of an abnormal P600 is broadly consistent with prior findings of abnormal P600 effects in stroke aphasia (Wassenaar et al., 2004) and agrammatic primary progressive aphasia (with the same task and materials; Barbieri et al., 2018) despite differences in how the abnormality has manifested across studies (delayed vs. reduced amplitude).

For the musical syntactic violations, the results from healthy controls replicate previous findings of P600 effects to musical syntactic violations (Patel et al., 1998; Lagrois, Peretz, \& Zendel, 2018). This is the first study to examine the P600 effect in response to musical syntactic violations in people with agrammatic aphasia. The finding of a larger P600 amplitude for patients than controls could be due to the patients' increased level of musical training compared to the healthy adults. Nevertheless, these findings suggest potentially intact online processing of musical syntax in agrammatic aphasia, at least in patients with $3+$ years of musical training. This initial finding is inconsistent with the predictions of the SSIRH, suggesting that musical and linguistic syntactic processing may be differentially impaired in agrammatic stroke aphasia. Results from the full study, which will include greater numbers of participants in both groups, will help to clarify the relation between music and linguistic syntax and how musical experience impacts this.

\section{References}

Patel, A. D. (2003). Language, music, syntax and the brain. Nature neuroscience, 6(7), 674.

Patel, A. D., Gibson, E., Ratner, J., Besson, M., \& Holcomb, P. J. (1998). Processing syntactic relations in language and music: An event-related potential study. Journal of cognitive 
neuroscience, 10(6), 717-733.

Kaan, E., Harris, A., Gibson, E., \& Holcomb, P. (2000). The P600 as an index of syntactic integration difficulty. Language and cognitive processes, 15(2), 159-201.

Friederici, A. D., Hahne, A., \& Saddy, D. (2002). Distinct neurophysiological patterns reflecting aspects of syntactic complexity and syntactic repair. Journal of psycholinguistic research, 31(1), 45-63.

Wassenaar, M., Brown, C. M., \& Hagoort, P. (2004). ERP Effects of Subject-Verb Agreement Violations in Patients with Broca's Aphasia. Journal of cognitive neuroscience, 16(4), 553-576.

Müllensiefen, D., Gingras, B., Musil, J., \& Stewart, L. (2014). The musicality of nonmusicians: an index for assessing musical sophistication in the general population. PloS one, 9(2), e89642.

Barbieri, E., Walenski, M., Litcofsky, K.A., Chiappetta, B., Hsu, C-J., Mesulam, M.M., \& Thompson, C.K. (May 2018). On-line sentence processing in Primary Progressive Aphasia. Clinical Aphasiology Conference. Austin, TX, USA.

Lagrois, M. É., Peretz, I., \& Zendel, B. R. (2018). Neurophysiological and behavioral differences between older and younger adults when processing violations of tonal structure in music. Frontiers in neuroscience, 12, 54. 


\title{
Brain metabolic correlates of errors on picture naming in Alzheimer's Disease
}

\author{
Cristina Rosazza ${ }^{1}$, Maria Gazzotti ${ }^{2}$, Paolo $\mathrm{Urso}^{2}$, Valentina Impagnatiello², Cinzia \\ Crivellaro $^{3}$, Valeria Isella ${ }^{2}$ \\ ${ }^{1}$ Fondazione IRCCS Istituto Neurologico Carlo Besta, Milan, Italy \\ ${ }^{2}$ Neurology Department, S. Gerardo Hospital, Monza, University of Milano - Bicocca, Italy \\ ${ }^{3}$ Nuclear Medicine, S. Gerardo Hospital, Monza, University of Milano - Bicocca, Italy
}

\section{Introduction}

Naming an object presented visually is a cognitively composite operation controlled by a vast network of brain areas. Patients with Alzheimer's Disease (AD) produce a variety of errors on confrontation naming that indicate impairment at multiple levels of the naming process. The precise topography of damage underlying such an impairment in AD is still controversial, with some studies showing extremely focal, left temporal, abnormalities, and others describing very widespread damage.

\section{Methods}

Subjects

Study participants were 70 patients referred to the memory clinic of the Department of Neurology of S. Gerardo Hospital, Monza, Italy, and meeting clinical criteria for probable AD. They were 37 women and 33 men and had a mean age of 71.7 years +8.1 (range: $50-85$ ), a mean education of 8.7 years +4.1 (range: 5 -19) and a mean score on the MiniMental State Examination of $23.3+3.4$ (range: 18-28), indicating mild to moderate disease severity.

\section{Naming assessment}

Confrontation naming was assessed with a standardized test comprising 80 stimuli of the Snodgrass and Vanderwart pictorial set. Total number of correct responses on the test was 4320 and mean number per patient was $61.7+13.9$ (range: 19-80). The following subtypes of naming errors were included in the subsequent imaging correlation analysis: anomias, circumlocutions, superordinates, coordinates, visual-semantic errors, pure visual errors, unrecognized stimuli and phonemic errors (including formal and phonological paraphasias). Their total number was 1225 and mean number per patient was $17.5+13.4$ (range: $0-61$ ).

\section{Brain FDG-PET analysis}

PET scans were acquired in the Department of Nuclear Medicine of S. Gerardo Hospital on a General Electric Discovery LS PET/CT scanner, reconstructed following an OSEM algorithm and subsequently processed with Statistical Parametric Mapping (SPM) 8. Correlation between brain metabolism and naming errors was carried out with linear regression analysis, including age, sex and MMSE score as covariates of no interest. Significance was set at $p<$ 0.05 FWE-corrected or $p<0.001$ uncorrected and only clusters with a minimum size of 100 voxels were taken into account.

\section{Results}

Anomias and Circumlocutions were associated with hypometabolism in the left ventral temporal cortex extending anteriorly for the former and posteriorly for the latter, but with a substantial overlap over the mid portion of the fusiform gyrus. A significant correlate emerged for Superordinates in the left parahippocampal gyrus, while Coordinates were associated with an area of hypometabolism including the posterior segments of the left superior and middle temporal gyri. The correlates of Visual-semantic and Pure visual errors encompassed the middle and 
inferior occipital gyri and posterior inferior temporal cortex in the left and right hemispheres, respectively. Unrecognized stimuli were associated with hypometabolism in the right inferior occipital-temporal cortex. Finally, a significant correlate emerged for Phonemic errors in the left hemisphere, along the posterior and mid portions of the superior temporal sulcus.

\section{Discussion}

Anomias and Circumlocutions were both related to the left ventral temporal lobe, a crucial area for semantic processing, suggesting that they are likely to be lexical-semantic in nature, in patients with AD. Superordinate errors were localized to the left entorhinal-perirhinal region, which is thought to have a critical role in distinguishing between semantically confusable items. Superordinates might thus arise from a deficit in activating distinctive properties of items belonging to a category. Coordinates were associated with left posterior superior temporal gyrus, in line with evidence mapping to this area the interface between semantics and phonology. Correlates of visual errors were reminiscent of the neuroanatomy of damage underlying apperceptive and associative agnosia, with Pure visual errors and Unrecognized stimuli linked to hypometabolism in the right occipital cortex and Visual-semantic errors to hypometabolism in the left occipital cortex. Phonemic errors were localized to the borders of the left superior temporal sulcus, which are known to be relevant for phonological processing, and short-term maintainance of phonemes in their correct sequence during repetition or lexical retrieval tasks. We believe that our findings fit generally well with dual-stream neurocognitive models of word generation and also contribute to their refinement providing some novel evidence.

\section{References}

Dell, G.S., Martin, N., Schwartz ,M.F. (2007). A case-series test of the interactive two-step model of lexical access: Predicting word repetition from picture naming. Journal of Memory and Language, 56, 490-520.

Hickok, G., Poeppel, D. (2007). The cortical organization of speech processing. Nature Reviews Neuroscience, 8, 393-402.

Humphreys, G. W., Riddoch, M. J. (1984). Routes to object constancy: implications from neurological impairments of object constancy. Quarterly Journal of Experimental Psychology, 36, 385-415.

Indefrey, P. Levelt, W.J.M. (2004). The spatial and temporal signatures of word production components. Cognition, 92, 101-144.

Laiacona, M., Barbarotto, R., Trivelli, C., Capitani, E. (1993). Dissociazioni semantiche intercategoriali: descrizione di una batteria standardizzata e dati normativi. Archivio Di Psicologia Neurologia e Psichiatria, 2, 209-248.

Lambon Ralph, M.A., Jefferies, E., Patterson, K., Rogers, T.T. (2016). The neural and computational bases of semantic cognition. Nature Reviews Neuroscience, 18, 42-55.

Verma, M., Howard, R.J. (2012). Semantic memory and language dysfunction in early Alzheimer's disease: A review. International Journal of Geriatric and Psychiatry, 27, 1209-1217.

Price, C.J. (2012). A review and synthesis of the first 20years of PET and fMRI studies of heard speech, spoken language and reading. Neuroimage, 62, 816-847.

Vigneau, M., Beaucousin, V., Hervéa, P., Duffau, H., Crivello, F., Houdé, O., TzourioMazoyer, N. (2006). Meta-analyzing left hemisphere language areas: Phonology, semantics, and sentence processing. Neuroimage, 30, 1414-1432.

Wright, P., Randall, B., Clarke, A., Tyler, L. K. (2015). The perirhinal cortex and conceptual processing: Effects of feature-based statistics following damage to the anterior temporal lobes. Neuropsychologia, 76, 192-207. 


\title{
When Trade-Offs in NeuroCognitive Resources Determine Word Production Efficiency in Aging
}

\author{
Ikram Methqal ${ }^{1,2}$, Yves Joanette ${ }^{1,2}$ \\ ${ }^{1}$ Centre de Recherche de l'Institut Universitaire de Gériatrie de Montréal, Canada \\ ${ }^{1,2}$ Université de Montréal, Canada.
}

\section{Introduction}

Healthy aging represents an adaptive process whereby behavioral performance is preserved through the recruitment of appropriate cognitive and neural resources in response to age-related brain changes and/or changes in strategies used to perform at a given task. Thus, maintaining cognitive performance in healthy aging does not mean acting youth-like but requires to be able to adopt strategies taking into consideration the task requirements (Reuter-Lorenz \& Cappell, 2008). Indeed, changes in the processing strategies used could be associated with evolutive and unique neurofunctional resources during healthy aging (Cabeza, 2002; Greenwood \& Parasuraman, 2010). In this respect, and because of their relative preservation in aging, language processing tasks provide unique insights into the dynamic evolutive neurofunctional changes in healthy aging.

Although numerous studies have explored the idea of age-related activation changes during language comprehension (Peelle et al., 2013; Wingfield \& Grossman, 2006), few have addressed neurofunctional reorganization issues in word production comparing younger and healthy older adults (Marsolais et al., 2015). Taken together, these studies suggest that bilateral frontal activations in older adults contribute to successful performance during comprehension tasks, but not always during production. The inconsistency posited by some neuroimaging findings illustrates the challenges associated with increased frontal activation as being the unique manner of improving verbal fluency performance in healthy aging. In this context, a verbal fluency task represent a reliable paradigm where individual performances can hinge on a self-determined strategy driven by trade-offs between exploitation (or clustering) and exploration (or switching) strategies, depending on the task requirement, yielding efficient performance with age (Hills et al., 2010). However, it remains valuable to explore whether age-related neurofunctional changes can be detected during the aforementioned verbal fluency tasks, which exert self- initiated strategies.

The goal of this study was to explore neurofunctional changes underlying the strategic processes in a verbal fluency task, comparing healthy older and younger adults, using an overt self-paced verbal fluency task paradigm.

\section{Methods}

Participants

Thirteen younger and 13 older participants underwent fMRI scanning. The fMRI protocol used an innovative mixed-designed procedure by which participants produced words during 90s in a natural self-paced manner, while fMRI whole head acquisitions were completed every 2 seconds and were then blocked according to task-related characteristics (Marsolais et al., 2015), allowing for blocks triggered by different events during the task. Thus, blocks were formed for trials when participants were asked to generate as many words starting with the same letter (L,P,M,V) or from the same semantic category (animals, vegetables, sports, clothes). Likewise, the design allowed to create blocks corresponding to clustering ( 2 or more consecutive words corresponding to a sub-set of the criterion) and/or switching (change between a given sub-set and another one) processes reflecting spontaneous and self-initiated strategies during VFT. In other words, this methodological approach allowed for valuable exploration of brain activity related to individual strategy productions (event-related activity), which could then also be 
grouped retrospectively in continuous blocks (block-related activity).

\title{
Results
}

Overall performance on both verbal fluency tasks were similar between younger and older participants (semantic, $p=.57$; orthographic, $p=.42$ ). However, in order to achieve similar performance, older adults provide more items in each sub-set thus producing significantly larger semantic clusters $(p<.05)$. This result suggests that older participants manage limited resources with a less costly cognitive strategy by producing more items for each cluster significantly larger semantic clusters significantly larger semantic clusters, as opposed to a more demanding switching, strategy. At a neurofunctional level, our main findings indicate that temporally mediated semantic clustering and frontally mediated orthographic switching were driven by evolutive neurofunctional reorganization in high-performing older adults. More specifically, our study revealed two main results: First, significant age-related activations during semantic clustering in inferior temporal regions, bilaterally for older adults as opposed to unilateral activation in the younger adult group (Figure 1).
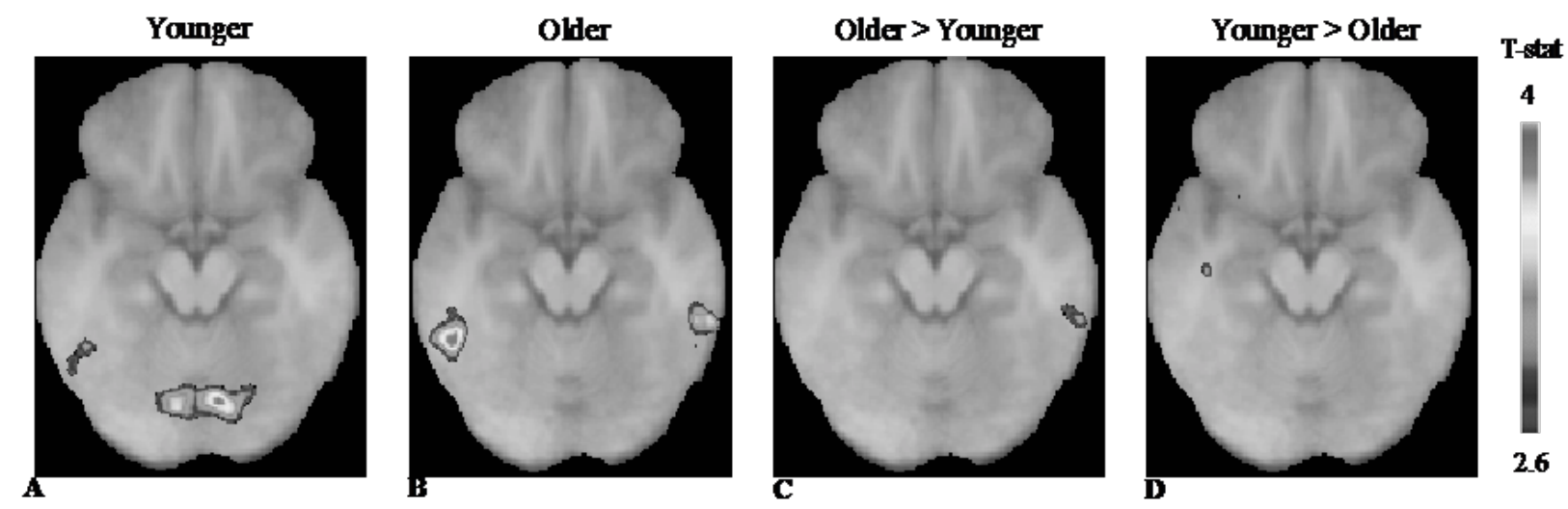

\begin{abstract}
Figure 1 | Activation for semantic chstering mins control condition. Honizontal section through the right inferior temporal cortex (area 37) peaks at $Z=-18$. The aveage of functional activity is superimposed on the anatomical MRI which is the average of the T1 acquisitions of the 13 younger adults and the 13 older adults transfomed into the Montreal Neurological Institute (MNI) standard proportional stereotaxic space. The color scale represents the T statistic. (A) Activity for the younger adults. (B) Activity for the older adults. (C) Activity for the older adults compared to the younger adults (uncomected at $\mathbf{p} 40.001$ ). (D) Activity for the younger adults compared to the older adults.
\end{abstract}

A significant activation during word production of semantic clusters in the left inferior temporal cortex (BA 37) was found for the older adults, while the left inferior prefrontal cortex (junction 6/8/44) and the left subcortical regions (left putamen and left caudate nucleus) were more relevant for the younger adults. Second, older adults showed bilateral frontal activations during orthographic-switching strategy, as opposed to unilateral activations for younger adults, to reach a high level of performance that leads to task success.

\section{Discussion}

The patterns of activation observed in the high-performing older participants in this study does not indicate that their performance are supported by youth-like brain activation patterns, but rather reflect the ability to maintain performance through the engagement of distinct cognitive strategies and shift neural activation to requisite brain regions. The change in strategy in older adults could reflect an old-age advantage of benefiting from a life-long improvement in verbal 
knowledge and expertise (Hedden and Gabrieli 2004). Hence, the changes in neural activity within semantic neural networks suggest that specific brain regions, such as the inferior temporal cortex, could provide a form of neurofunctional scaffolding for high-performing healthy older adults, allowing them to cope with lowered prefrontal and caudate activity. In line with the STAC model (Park \& Reuter-Lorenz, 2009), the neurofunctional scaffolds built up through life experiences and acquired knowledge might be critical to shrink a gap between behavioral and functional patterns to better maintain lexico-production ability in healthy high-performing older adults, as well as adopting appropriate and advantageous strategies to support the fulfillment of task demands.

Beyond the maintenance of a good orthographic fluency performance in older and younger adults, our findings also suggest that the switching strategy may have been more challenging for the older participants because of a reduction in left frontal activations. Overall, this study shows that the exploration of neurofunctional changes underlying the strategic processes in a verbal fluency task informs both about the functioning of the aging brain as well as its ability to manage trade-offs between cognitive and neural resources to successfully perform. Thus, maintaining performance in healthy aging does not mean acting youth-like, but rather expresses the ability to adopt strategies based on task requirements, as long as resources continue to be available.

\section{References}

Cabeza, R. (2002). Hemispheric asymmetry reduction in older adults: The HAROLD model. Psychology and Aging, 17(1), 85-100.

Greenwood, P. M., \& Parasuraman, R. (2010). Neuronal and cognitive plasticity: A neurocognitive framework for ameliorating cognitive aging. Frontiers in Aging Neuroscience, 2(NOV), 1-14.

Hedden, T., \& Gabrieli, J. D. E. (2004). Insights into the ageing mind: A view from cognitive neuroscience. Nature Reviews. Neuroscience, 5(2), 87-96.

Hills, T. T., Todd, P. M., \& Goldstone, R. L. (2010). The Central Executive as a Search Process: Priming Exploration and Exploitation Across Domains. Journal of Experimental Psychology: General, 139 (4), 590-609.

Marsolais, Y., Methqal, I., \& Joanette, Y. (2015). Marginal neurofunctional changes in highperform- ing older adults in a verbal fluency task. Brain and Language, 140, 13-23.

Park, D. C., \& Reuter-Lorenz, P. (2009). The adaptive brain: Aging and neurocognitive scaffolding. Annual Review of Psychology, 60(1), 173-196.

Peelle, J. E., Chandrasekaran, K., Powers, J., Smith, E. E., \& Grossman, M. (2013). Agerelated vulnerability in the neural systems supporting semantic processing. Frontiers in Aging Neuroscience, 5(SEP), 1-11.

Reuter-Lorenz, P. A., \& Cappell, K. A. (2008). Neurocognitive aging and the compensation hypothesis. Current Directions in Psychological Science, 17(3), 177-182.

Wingfield, A., \& Grossman, M. (2006). Language and the aging brain: Patterns of neural compensation revealed by functional brain imaging. Journal of Neurophysiology, 96(6), 2830-2839. 


\title{
Written Language in Mandarin-dominant Older Adults with Hearing Loss
}

\author{
Liyana Low ${ }^{1}$, Susan J. Rickard Liow ${ }^{2}$, Melvin J. Yap ${ }^{3}$, Tng Siok Keng ${ }^{2}$, Rebecca Heywood ${ }^{4}$ \\ ${ }^{1}$ Masters of Science (Speech and Language Pathology), Division of Graduate Medical Studies, \\ Yong Loo Lin School of Medicine, National University of Singapore \\ ${ }^{2}$ Department of Otolaryngology, Yong Loo Lin School of Medicine, National University of \\ Singapore \\ ${ }^{3}$ Department of Psychology, Faculty of Arts and Social Sciences, National University of \\ Singapore \\ ${ }^{4}$ Department of Otolaryngology, Ng Teng Fong General Hospital, Singapore
}

\section{Introduction}

Age-related hearing loss is the most common acquired communication disability affecting older adults (Hickson \& Scarinci, 2007), and is strongly related to cognitive impairment (Heywood et al., 2017; Lin et al., 2011; Loughrey, Kelly, Kelley, Brennan \& Lawlor, 2017). The impact of this relationship on the written language abilities, especially for those who speak languages involving lexical tone, warrants investigation given the increased use of internet and social media amongst older adults (Pew Research Centre, 2017). The aim of this study was to examine the relationship between hearing loss, cognitive abilities, and written language skills in Mandarindominant 60-80 year-olds using Taft, Liu and Zhu's (1999) Interactive Activation Framework for possible mechanisms underlying written language processing in Chinese.

\section{Methods}

Literate Mandarin-dominant older adults (mean age 70.34 years) with normal hearing $(\mathrm{n}=15)$ or hearing loss $(n=7)$ were assessed using a locally developed Chinese Reading and Spelling Assessment battery comprising word naming (60 items), lexical decision task (120 items) and spelling (120 items). Phonetic-compound Chinese characters were used in the assessment battery. Semantic picture matching, written word to picture matching and written picture naming tests were administered to identify possible semantic impairments. Hearing loss was measured by pure-tone audiometry and cognitive abilities were gauged using the Montreal Cognitive Assessment (MOCA).

\section{Results}

Following a partial correlation analysis (controlling for age and education), the data showed a significant negative correlation between hearing loss and written picture naming accuracy, and a borderline significant positive association between cognition (MOCA scores) and spelling performance. When participants were matched for age and education, the qualitative analysis of error patterns revealed that those with hearing loss made two types of atypical spelling errors. First, they produced spellings that were completely different visually from the original target and with a different tone, although they shared syllable phonology, e.g., Target: 猜 (cai1), Response: 采 (cai3). Second, they produced spelling responses that contained the target's phonetic radical but the phonology of the written character was either the same as that of the target but had a different tone, e.g., Target: 炒 (chao3), Response: 抄 (chao1), or completely different from the original target, e.g., Target: 吩 (fen1), Response: 扮 (ban4). The second set of errors were produced mostly for low-frequency group of targets.

\section{Discussion}

If phonological activation plays a role in written processing of Chinese (Damian \& Qu, 2013; Guo, Peng, \& Liu, 2005; Tan \& Perfetti, 1999), and phonological processing is represented in the 
left middle and superior temporal gyri (Bookheimer, Zeffiro, Blaxton, Gaillard, \& Theodore, 1995; Demonet et al., 1992), it follows that neural degradation in these brain areas as a result of presbycusis (Chen et al., 2016) might contribute to the selection of the wrong phonological representation in Mandarin-dominant adults with hearing loss.

Our sample size is limited, but the quantitative and qualitative results suggest that hearing loss and subsequent cognitive impairment in older adults who use tonal languages, such as Mandarin, can have a negative impact on written language processing. The data appear consistent with Lin et al.'s (2014) suggestion that hearing loss is associated with accelerated brain atrophy, especially in the temporal lobe structures, an area suggested to be important not only for spoken language processing (Peelle, 2012) but is also reported into be involved with semantic memory and the early stages of mild cognitive impairment (Chetelat et al., 2005; Tranel, Damasio \& Damasio, 1997)

With reference to the quantitative analysis, based on Taft et al.'s (1999) framework, the activation of an incorrect phonological representation arising from reduced phonological processing abilities in those with hearing loss would lead to the selection of an incorrect orthographic representation. This sequence of events would explain why the older adults with hearing loss achieved significantly lower written naming scores. With reference to the qualitative analysis of errors, reduced phonological processing abilities in participants with hearing loss could also contribute to the inaccurate selection of characters with phonologies that were completely different from the expected target, or had different tones from the target.

\section{References}

Bookheimer, S. Y., Zeffiro, T. A., Blaxton, T., Gaillard, W., \& Theodore, W. (1995). Regional cerebral blood flow during object naming and word reading. Human Brain Mapping, 3(2), 93-106.

Chetelat, G., Landeau, B., Eustache, F., Mezenge, F., Viader, F., De La Sayette, V., ... \& Baron, J. C. (2005). Using voxel-based morphometry to map the structural changes associated with rapid conversion in MCI: a longitudinal MRI study. Neuroimage, 27(4), 934-946.

Chen, X., Wang, M., Deng, Y., Liang, Y., Li, J., \& Chen, S. (2016). Language processing of auditory cortex revealed by functional magnetic resonance imaging in presbycusis patients. Acta oto-laryngologica, 136(2), 113-119.

Damian, M., \& Qu, Q. (2013). Is handwriting constrained by phonology? Evidence from Stroop tasks with written responses and Chinese characters. Frontiers in psychology, 4, 765.

Demonet, J. F., Chollet, F., Ramsay, S., Cardebat, D., Nespoulous, J. L., Wise, R., ... \& Frackowiak, R. (1992). The anatomy of phonological and semantic processing in normal subjects. Brain, 115(6), 1753-1768.

Guo, T., Peng, D., \& Liu, Y. (2005). The role of phonological activation in the visual semantic retrieval of Chinese characters. Cognition, 98(2), B21-B34.

Heywood, R., Gao, Q., Nyunt, M. S. Z., Feng, L., Chong, M. S., Lim, W. S., ... \& Ng, T. P. (2017). Hearing loss and risk of mild cognitive impairment and dementia: findings from the Singapore Longitudinal Ageing Study. Dementia and geriatric cognitive disorders, 43(5-6), 259-268.

Lin, F. R., Metter, E. J., O’brien, R. J., Resnick, S. M., Zonderman, A. B., \& Ferrucci, L. (2011a). Hearing loss and incident dementia. Archives of neurology, 68(2), 214-220.

Lin, F. R., Ferrucci, L., An, Y., Goh, J. O., Doshi, J., Metter, E. J., ... \& Resnick, S. M. (2014). Association of hearing impairment with brain volume changes in older adults. 
Neuroimage, 90, 84-92.

Loughrey, D. G., Kelly, M. E., Kelley, G. A., Brennan, S., \& Lawlor, B. A. (2017). Association of age-related hearing loss with cognitive function, cognitive impairment, and dementia: a systematic review and meta-analysis. JAMA Otolaryngology-Head $\&$ Neck Surgery.

Peelle, J. E. (2012). The hemispheric lateralization of speech processing depends on what "speech" is: a hierarchical perspective. Frontiers in Human Neuroscience, 6, 309.

Pew Research Center. (2017) Tech Adoption Climbs Among Older Adults. Retrieved December 1, 2018 from http://www.pewinternet.org/2017/05/17/technology-use-among-seniors/

Taft, M., Liu, Y., \& Zhu, X. (1999). Morphemic processing in reading Chinese. In J. Wang, A. Inhoff \& H. C. Chen (Eds.), Reading Chinese script: A cognitive analysis (pp. 91113). Mahwah, NJ: Lawrence Erlbaum.

Tan, L. H., \& Perfetti, C. A. (1999). Phonological activation in visual identification of Chinese two-character words. Journal of Experimental Psychology: Learning, Memory, and Cognition, 25(2), 382.

Tranel, D., Damasio, H., \& Damasio, A. R. (1997). A neural basis for the retrieval of conceptual knowledge. Neuropsychologia, 35(10), 1319-1327. 


\title{
Determining the ideal length of spontaneous speech fragments for predictive analysis
}

\author{
Roelant Ossewaarde ${ }^{1,2}$, Roel Jonkers ${ }^{1}$, and Roelien Bastiaanse ${ }^{1}$ \\ ${ }^{1}$ Center for Language and Cognition Groningen (CLCG), Rijksuniversiteit Groningen, \\ Netherlands \\ ${ }^{2} H U$ University of Applied Science, Utrecht, Netherlands
}

\section{Introduction}

Spontaneous speech is an important source of information for aphasia research (Prins and Bastiaanse 2004). It is essential to collect the right amount of data: enough for distinctions in the data to become meaningful, but not so much that the data collection becomes too expensive or places an undue burden on participants. The latter issue is an ethical consideration when working with participants that find speaking difficult, such as speakers with aphasia.

The influence of dementia on language production can be quantified using statistical methods applied to a corpus of spontaneous speech. A trained statistical model can predict group membership (speakers with dementia or non-brain damaged speakers) based on speech transcripts (Fraser, Rudzicz, and Rochon (2013) inter alia). Such approaches require training data that are sufficiently abundant for a model to reliably detect difference. Abundancy of data is a function of two aspects: enough training instances (conversations) and enough distributional difference between the variables of the model. Distributional difference is obtained by collecting conversations long enough to reliably quantify variables of interest. After analyzing a set amount of conversation, a parameter can be reliably estimated, and as a consequence the cost of analysis starts to outweigh the benefit of collecting more data. There are different norms on how much data to collect. For example, Nicholas and Brookshire (1993) and Vermeulen, Bastiaanse, and Van Wageningen (1989) base their analyses on the first 300 words of a fragment. Some clinicians use a set amount of time, rather than words, if the speech rate is low or severely nonfluent. It is an open question whether text length at all is influential for interpreting the machine learned models that are used to classify speech fragments into diagnostic groups.

We present a case study of how the minimally required text length for a given corpus can be determined so that models can be construed which can make meaningful discriminations based on the predictor parameters. It is inspired by the questions that arose while determining parameters (required text length) for a future longitudinal study. We compare the results of our analyses with existing analyses of the same data.

\section{Methods}

The core of this study is the estimation of the uncertainty around variables in fragments of different text length. We train statistical models of variables on the word and sentence levels to determine the relation between text length and uncertainty. The training data is processed by software (Stanford Parser) that can determine sentence and word level variables in fragments with a given length. As training data, we use two corpora of conversations with individuals with aphasia: (1) English speakers in the Pitt corpus of the DementiaBank clinical dataset (Becker et al. 1994), and (2) German speakers in data collected for a larger study of processing of verbs and nouns in speakers with different types of dementia.

In general, uncertainty in a trained model can decrease when (a) differences between measurements become larger, when (b) the certainty of individual measurements increases, or when (c) the amount of training data available increases. In our context, increasing text length or 
collecting data from more individuals may be used as strategies for (b) and (c). This study investigated (b).

Bayesian modeling was applied with as parameters several word and sentence level variables that are often used in brain damaged vs non brain damaged classification tasks. The values of parameters and their uncertainty were estimated through Hamiltonian Monte Carlo simulation using STAN and R. We chose Bayesian modeling because the method does not depend on large samples, and because the Bayesian interpretation of probability provides a natural estimation of the relation between model uncertainty and text length. The resulting models are compared to a model that includes only age as predictor, to quantify how a model with linguistic variables fares better than a model without them. The model maps parameters onto a binomial distribution using the logit link function. All parameters (except 'age') were normalized to the mean and scaled around their standard deviation.

\section{Results}

The Markov chains converged, with no orphaned or clumpy chains.

There is a similar pattern of decreasing uncertainty for four of six linguistic variables that are often used in predictive models. In this study, observations in a range of 0-4 units of SD were observed. A model with predictor variables that deviate within that range can be trained with 500 words or more. The informative gain starts to diminish when sample length increases beyond 1100 words. The uncertainty around the predictor variables in samples with less than 500 words becomes so large that measurements would have to have unrealistic deviations in order to become significant, at least in the context of this corpus. The different variables converge to a surprisingly similar degree to become predictive at the same cutoff in terms of sample length, at around 700 words. Spontaneous speech samples of that length are long enough to classify brain damaged and non brain damaged speakers with the variables of this study.

\section{References}

Becker, James T, François Boiler, Oscar L Lopez, Judith Saxton, and Karen L McGonigle. 1994. "The Natural History of Alzheimer's Disease: Description of Study Cohort and Accuracy of Diagnosis." Archives of Neurology 51 (6). American Medical Association: 585-94.

Fraser, Kathleen C, Frank Rudzicz, and Elizabeth Rochon. 2013. "Using text and acoustic features to diagnose progressive aphasia and its subtypes." Interspeech, 2177-81.

Nicholas, L E, and R H Brookshire. 1993. "A System for Quantifying the Informativeness and Efficiency of the Connected Speech of Adults with Aphasia." J Speech Hear Res 36 (2): $338-50$.

Prins, Ronald and Roelien Bastiaanse (2004) "Review Analysing the spontaneous speech of aphasic speakers", Aphasiology, 18:12, 1075-1091.

Vermeulen, J, R Bastiaanse, and B Van Wageningen. 1989. "Spontaneous Speech in Aphasia: A Correlational Study." Brain Lang 36 (2): 252-74. 


\title{
Short Term Memory and sentence processing in deep dysphasia
}

\author{
Xabi Ansorena ${ }^{1}$, Mireia Hernández ${ }^{2,3}$, Manuel Carreiras ${ }^{1}$, José Ignacio Quemada ${ }^{4}$, Simona \\ Mancini $^{1}$ \\ ${ }^{1}$ Basque Center on Cognition, Brain and Language, BCBL, Donostia, Spain \\ ${ }^{2}$ Section of Cognitive Processes, Department of Cognition, Development, and Educational \\ Psychology, Institut de Neurociències, Universitat de Barcelona, Barcelona, Spain \\ ${ }^{3}$ Cognition and Brain Plasticity Group, Bellvitge Biomedical Research Institute (IDIBELL), \\ L'Hospitalet de Llobregat, Barcelona, Spain \\ ${ }^{4}$ Jefe de Servicio de Daño Cerebral, Hospital Aita Menni, Mondragón, País Vasco
}

\section{Introduction}

Deep dysphasia refers to a rare form of conduction aphasia (Katz and Goodglass, 1980; Michel \& Andreewski, 1983; Majerus, 2001) characterized by (a) an impaired auditory Short Term Memory (STM) span, (b) lexicality effects, showing better repetition of words than pseudowords, (c) imageability effects, with better repetition of high imageability words than low imageability words and (d) semantic errors.

Katz and Goodglass (1990) first conceptualized this disorder as a result of damage to two different routes: damage to the semantic-lexical route accounted for semantic errors and imageability effects, while damage to the nonsemantic lexical and nonlexical route accounted for lexicality effects. Others (Ablinger et al. 2008; Martin and Saffran, 1992; Martin et al. 1994) have proposed interactive spreading activation models that explain these symptoms as a global decay of the input trace in the STM.

Existing studies of deep-dysphasic patients have primarily reported word-repetition deficits, while sentence repetition impairments are mostly absent from these studies (but see Saffran \& Marin, 1975). Here, we report the case of a patient with deep dysphasia that also shows impairment in the repetition and comprehension of sentences. In this study we deepen our understanding of the sentence repetition impairment in deep dysphasia and discuss its relation with STM and cognitive models accounting for this syndrome.

\section{Methods}

Case description

$\mathrm{KG}$ is a 61-year old, right-handed Spanish monolingual man who suffered from an ischemic infarction of the right Middle Cerebral Artery ${ }^{2}$ causing him right temporoparietal damage 12 months ago. He has 20 years of education and, at the time of the neurological event, he had worked as a nurse for 35 years.

Audiological examination showed no abnormality. Neuropsychological assessment revealed a STM impairment, by both The Digit Span Task (Direct Span=4, percentile range 3-5; Inverse Span=3, percentile range 29-40) and the Auditory Verbal Learning Test $[1(-3.75) / 3(-3.09) /$ $5(-3.1) / 6(-2.91) / 4(-4.42)$, z scores in parenthesis]. Linguistic assessment (BETA: Cuetos \& Gonzalez, 2009) showed good naming (78/80) and lexical access (150/160), while the scores in repetition, grammaticality judgment task and the Token Test were low, particularly after auditory input.

\footnotetext{
${ }^{2}$ Because deep dysphasia symptoms are normally associated with left-hemiphere damage, language lateralization testing is ongoing for $\mathrm{KG}$
} 
Even if word repetition was unimpaired, a significant drop in accuracy emerged in pseudoword repetition [Lexicality effect: $2_{(1)}=1681, p<0.001$ ], especially after auditory input. In sentence repetition, we observed semantic errors [e.g. chica (girl) $\rightarrow$ mujer (woman)]) as well as morphological [perseguir (to chase) $\rightarrow$ seguir (follow)] and syntactic simplifications [La chica es vista por la gallina (The girl is seen by the hen) $\rightarrow$ La gallina ha visto a la chica (The hen has seen the girl)]. To test for imageability effects, we administered a single-word repetition task manipulating imageability. Results showed significantly lower accuracy for low-imageable compared to high-imageable words, although only in dictation $\left[2_{(1)}=3.96, p<0.05\right]$.

\section{Materials and procedure}

To investigate KG's performance at the sentence level and the auditory-visual input dissociation more in depth, additional comprehension and repetition tasks were administered. In a sentencepicture matching task, 20 sentences were presented to be associated with their corresponding target picture, intermixed with three distractors. The stimuli included active, relative-clause, passive and subject/object-focalized sentences (see examples below).

1. La jirafa es golpeada por el elefante

The giraffe is beaten by the elephant

2. Es al cerdo al que muerde la oveja

It is the pig that the sheep is biting

3. El niño se ríe del payaso que lleva peluca

The boy laughs at the clown who wears a wig

Sentences were administered both visually and auditorily. We also administered these same sentences in an additional repetition task. All tasks are described and their scores summarized in Table 1.

\section{Results}

For the sentence picture matching task, performance in both auditory and visual input was impaired. KG showed more difficulties in object focalized (1/8) and passive $(3 / 8)$ sentences as compared to active (5/8), relative (5/8) and subject-focalized (8/8) sentences. Interestingly, some errors involved thematic role inversion. For example, for sentence (1), the choice of the patient would be a picture in which the giraffe beats the elephant.

In repetition, performance on visual tasks (reading+written copy) was better than performance on auditory tasks (oral repetition+dictation) both globally [ $2(1)=500.13, p<0.001]$ and when comparing individual tasks, as shown in table 1. Qualitative analysis of repetition errors evidenced omissions (15\%), additions (4\%), substitutions (25\%), phonological paraphasias (15\%), semantic errors $(23 \%)$, and simplifications of syntactic structure (19\%). Interestingly, these percentages were distinct in the auditory and the visual input, with a higher percentage of semantic errors in the auditory input (27\% vs. $0 \%)$ and a higher percentage of phonological errors in the visual input ( $28 \%$ vs. $12 \%)$.

\section{Discussion}

Overall, the data reported here show that impairment in deep dysphasia goes beyond the singleword level, extending to sentence repetition and comprehension, as shown by KG's semantic substitutions and simplification of syntactic structure, as well as his reduced accuracy in the Sentence Picture Matching tasks. The consistent dissociation in performance found after visual and auditory input, as well as KG's poor performance in digit-span and RAVLT tests, point to a main deficit in auditory STM. 


\begin{tabular}{|c|c|c|c|c|c|c|c|}
\hline Stimuli & Task description & Auditory tasks (1) & $\begin{array}{c}\text { Score } \\
(1)\end{array}$ & $\begin{array}{c}\text { Score } \\
(2)\end{array}$ & Visual tasks (2) & $\begin{array}{c}\chi^{2} \\
\text { value }\end{array}$ & p value \\
\hline Phonemes & $\begin{array}{c}\text { Pairs of stimuli, half of which differ in a phoneme, taken from BETA (Cuetos- } \\
\text { Vega \& González Nosti, 2009). The patient must decide if they are equal or } \\
\text { different. Equally divided in words and pseudowords }\end{array}$ & $\begin{array}{l}\text { Auditory phonemic } \\
\text { discrimination }\end{array}$ & $31 / 32$ & $31 / 32$ & $\begin{array}{l}\text { Written phonemic } \\
\text { discrimination }\end{array}$ & 0 & ns \\
\hline \multirow{3}{*}{ Words } & $\begin{array}{l}\text { Half words and pseudowords are presented and the patient decides if they } \\
\text { are real or not. Words are names (animals and tools) and verbs of high and } \\
\text { low frequency. Pseudowords are extracted from these words using Wiggy } \\
\text { (Keuleers \& Brysbaert, 2010) }\end{array}$ & Auditory lexical access & $75 / 80$ & $75 / 80$ & Written lexical access & 0 & ns \\
\hline & Repetition of verbs and nouns of high and low frequency and imageability & Word repetition oral & $35 / 40$ & $38 / 40$ & Word repetition reading & 36 & $<.05$ \\
\hline & Repetition of verbs and nouns of high and low frequency and imageability & $\begin{array}{l}\text { Word repetition } \\
\text { dictation }\end{array}$ & $26 / 40$ & $36 / 40$ & $\begin{array}{l}\text { Word repetition written } \\
\text { copy }\end{array}$ & 100 & $<.01$ \\
\hline \multirow{2}{*}{ Pseudowords } & Pseudowords extracted from the words in the repetition task. Using Wiggy & $\begin{array}{l}\text { Pseudoword repetition } \\
\text { oral }\end{array}$ & $11 / 40$ & $37 / 40$ & $\begin{array}{l}\text { Pseudoword repetition } \\
\text { reading }\end{array}$ & 676 & $<.001$ \\
\hline & Pseudowords extracted from the words in the repetition task. Using Wiggy & $\begin{array}{l}\text { Pseudoword repetition } \\
\text { dictation }\end{array}$ & $6 / 40$ & $37 / 40$ & $\begin{array}{l}\text { Pseudoword repetition } \\
\text { written copy }\end{array}$ & 961 & $<.001$ \\
\hline \multirow{5}{*}{ Sentences } & $\begin{array}{l}\text { The token test (De Renzi and Vignolo, 1962) is a well-known test that } \\
\text { assesses comprehension of commands of increasing complexity* }\end{array}$ & Auditory Token Test & $46 / 62$ & $36 / 36$ & Written Token Test & 138.182 & $<.001$ \\
\hline & $\begin{array}{l}\text { Consists of sentences, half of which are grammatically correct and half are } \\
\text { incorrect. The patient judges their correctness }\end{array}$ & $\begin{array}{l}\text { Auditory grammaticality } \\
\text { judgment }\end{array}$ & $30 / 40$ & $36 / 40$ & $\begin{array}{l}\text { Written grammaticality } \\
\text { judgment }\end{array}$ & 36 & ns \\
\hline & $\begin{array}{l}\text { Active, passive, object focused and subject focused sentences are presented } \\
\text { and the patient chooses one of four pictures that corresponds to these } \\
\text { sentences. Grammatical and semantic distractors are included }\end{array}$ & $\begin{array}{l}\text { Auditory sentence } \\
\text { picture matching }\end{array}$ & $12 / 20$ & $10 / 20$ & $\begin{array}{l}\text { Written sentence picture } \\
\text { matching }\end{array}$ & 4 & ns \\
\hline & Same sentences from the Sentence Picture Matching task & Sentence repetition oral & $5 / 20$ & $19 / 20$ & $\begin{array}{l}\text { Sentence repetition } \\
\text { reading }\end{array}$ & 196 & $<.001$ \\
\hline & Same sentences from the Sentence Picture Matching task & $\begin{array}{l}\text { Sentence repetition } \\
\text { dictation }\end{array}$ & $3 / 20$ & $15 / 20$ & $\begin{array}{c}\text { Sentence repetition } \\
\text { written copy }\end{array}$ & 144 & $<0.001$ \\
\hline
\end{tabular}

Table 1 describes the tasks that were compared for auditory (1) and visual (2) input; and shows the scores obtained by KG. The tasks are different in the type of stimuli (phonemes, words, pseudowords, and sentences). For the individual comparisons of auditory and visual input, we provide $\chi 2$ and $p$ values. All $\chi 2$ values are calculated with one degree of freedom

*Two different versions of the Token Test were administered. This was because for the second (visual) assessment we noticed that the short version (De Renzi \& Faglioni, 1978) has better norms for Spanish

We tentatively discuss these data in relation to existing models of deep dysphasia that propose interactive spreading activation at the phonological, semantic and lexical level. In the presence of auditory input, the phonological level is the first to be activated, but a STM impairment causes this trace to decay rapidly, making selection at the lexical level (including syntactic information) to rely solely on semantic information. This causes repetition errors, such as the semantic substitution of girl with woman, which KG produced in sentence repetition. Interestingly, a similar explanation holds also for the syntactic simplifications produced during sentence repetition. In this case, the rapid decay of the phonological trace forces the system to rely on semantic information, whereby an output with simpler syntactic structure but equivalent meaning is selected (the girl is seen by the hen $\rightarrow$ the hen sees the girl). This is in line with recent reports on the effects of STM impairment on sentence processing (Papagno, \& Cecchetto, 2018).

Further testing is underway with behavioral and neuro-imaging techniques to better characterize the sentence-level impairment observed.

\section{References}

Ablinger, I., Abel, S. \& Huber, A. (2008). Deep dysphasia as a phonetic input deficit: Evidence from a single case. Aphasiology, 22(5), 537-556.

Cuetos-Vega, F., \& González-Nosti, M. (2009). Batería para la Evaluación de los Trastornos Afásicos (BETA). Madrid: Instituto de orientación psicológica EOS.

De Renzi, E., \& Vignolo, L. (1962). The Token Test: A sensitive test to detect receptive disturbances in aphasics. Brain, 85, 665-678.

Majerus, S., Lekeu, F., Van der Linden, M., \& Salmon, E. (2001). Deep dysphasia: Further evidence on the relationship betweenphonological short-term memory and language 
processing impairments. Cognitive Neuropsychology, 18(5), 385-410.

Martin, N., \& Saffran, E. M. (1992). A computational account of deep dysphasia: Evidence from a single case study. Brain and Language, 43(2), 240-274.

Martin, N., Dell, G. S., Saffran, E. M., \& Schwartz, M. F. (1994). Origins of Paraphasias in Deep Dysphasia: Testing the Consequences of a Decay Impairment to an Interactive Spreading Activation Model of Lexical Retrieval. Brain and Language, 47(4), 609-660.

Martin, R. C. (1990). Neuropsychological evidence on the role of short-term memory in sentence processing. In G. Vallar \& T. Shallice (Eds.), Neuropsychological impairments of short-term memory (pp. 390-427). New York, NY, US: Cambridge University Press.

Martin, R. C., Lesch, M. F., \& Bartha, M. C. (1999). Independence of input and output phonology in word processing and short-term memory. Journal of Memory and Language, 41, 3-29.

Michel, F., \& Andreewsky, E. (1983). Deep Dysphasia: An Analog of Deep Dyslexia in the Auditory Modality. Brain and Language, 18, 212-223.

Miranda, J. P., \& Valencia, R. R. (1997). English and Spanish versions of a memory tests: Word-length effects versus spoken duration effects. Hispanic Journal of Behavioral Science, 19, 171-181.

Papagno, C., Cecchetto, C. (2018). Is STM involved in sentence comprehension? Cortex, 112, 80-90.

Saffran, E.M., \& Marin, O.S. (1975). Inmediate memory for word lists and sentences in a patient with deficient auditory short term memory. Brain and Language, 2(4), 420-433.

Sidiropoulos, K., Bormann, T. \& Ackermann, H. (2014). Cortical and fibre tract interrelations in conduction aphasia, Aphasiology, 28(10), 1151-1167

Katz, B., \& Goodglass, H. (1990). Deep Dysphasia: Analysis of a Rare Form of Repetition Disorder. Brain and Language, 39(1), 153-185.

Keuleers, E., \& Brysbaert, M. (2010). Wuggy: A multilingual pseudoword generator. Behavior Research Methods 42(3), 627-633.

Wechsler, D. (2004). Escala de memoria de Wechsler-III. Manual de aplicación y puntuación. Madrid: TEA Ediciones. 


\title{
Language abilities in Aicardi Syndrome: A case study
}

\author{
Soultana Georgiadou, Stavroula Stavrakaki, Vasileios Kimiskidis \\ Aristotle University of Thessaloniki, Greece
}

\section{Introduction}

Aicardi syndrome (AS) is a rare neurodevelopmental disorder which occurs in 1 out of 105.000 births (Shirley et al. 2016; Lund et al., 2015). Two of the following clinical criteria are required for the AS diagnosis: (i) partial or total callosal agenesis; (ii) infantile spasms; (iii) chorioretinal lacunae (Willis \& Rosman, 1980). In addition, two of the following clinical features are expected to be present in individuals with AS : i) cortical malformations; ii) bladder around the third ventricle; iii) bladder in the chorea grid; iv) optic nerve cross section; v) subcortical heterotopia (Barkovich et al., 2005; Prinzivalli et al., 2013; Saini et al., 2018; Sutton et al., 2005). AS is not familial and occurs only in individuals with two $\mathrm{X}$ chromosomes. Individuals with AS show ophthalmological and musculoskeletal deficiencies while they quite often suffer from psychiatric problems (Cabrera et al., 2011; Saddichha et al., 2007; Tuft et al., 2017). In addition, they are remarkably impaired in cognitive abilities and show cognitive decline over time (Grosso et al. 2007; Zaarour \& Traboulsi, 2017).

Few studies have been performed on the language abilities of AS which reveal controversial findings. On the one hand, some researchers report significant impairment in language. In particular, reduced expressive abilities have been attested in AS as shown by short speech length and limited word number production. In addition, syntactic abilities have been affected as shown by grammatical errors (Banerjee et al., 2006; Menezes et al., 1994; Merrick, 2009). Deficits in receptive language abilities have also been reported as indicated by difficulties in following complex instructions (Jain \& Dinesh, 2016; Tuft et al., 2017). On the other hand, other researchers report intact language abilities (Saddichha et al., 2007). In addition to the studies on AS cases, other studies have investigated the role of corpus callosum in language performance by testing individuals with agenesis of corpus callosum (ACC). These studies which have reported controversial findings for the linguistic abilities of individuals with ACC are quite relevant to the studies of AS individuals with partial or total callosal agenesis (see for example, Jeeves \& Temple, 1987; Lassonde, Bryden, \& Demers, 1990; Rehmel et al., 2016). The present study aims at exploring the language abilities in AS by investigating the aspects of oral and written language abilities of an individual with AS (with total callosal agenesis). In addition, it raises the question of whether the callosal agenesis is required for the typical development of specific language functions.

\section{Methods}

\section{Patient}

The patient JH (female) was diagnosed with AS at 6 years old by a neurologist. MRI data has showed that she has total callosal agenesis but anterior and posterior commissures have been present. At the time of testing she has been 23 years old. She is right-handed and lives with her family. She has completed 12 years of education in the technical domain while she has currently been a student of a technical college for students with special educational needs. The patient has particular vision difficulties and thus she wears myopia glasses.

Tools

We employed the following materials to assess AS's cognitive and language abilities: 
Cognitive abilities

- Wechsler Adults Intelligence Scale (WAIS-IV ${ }^{\mathrm{GR}}$ ) (Stogiannidou, 2014) for IQ assessment.

Oral language abilities

- The Greek version of the semantic and phonological fluency test (Kosmidis et al., 2004) for word retrieval ability assessment in the semantic and phonological domain.

- The Perfective Past Tense Test for Greek speaking population (Stavrakaki \& Clahsen, 2009). For the purposes of this study the existing regular and irregular part was employed.

In addition, to evaluate her spontaneous speech production, a 5-minute conversational speech recording was performed.

Written language abilities

- The lexical decision task developed by the Lab of Cognitive Neuroscience, School of Psychology, Aristotle University of Thessaloniki (AUTh) (2018) to investigate whether the participant was able to make a decision on the correct vs. incorrect word orthography of the presented stimuli.

- The orthographic test for adolescents and adults: Regular and irregular words are orally presented and the participant has to write them down (regular and irregular words) (Lab of Cognitive Neuroscience, School of Psychology, AUTh, 2018).

\section{Results}

AS patient showed mild cognitive impairment with a total IQ score of 62 in WAIS-IVGR Spontaneous speech analysis revealed that her speech was characterised by short but totally comprehensive and grammatical utterances. With respect to the other measures, impaired performance was considered as the one which was 2 Standard Deviations (SDs) below typical mean (as it is reported for adult performance on the relevant testing materials). Table 1 shows ceiling performance on past tense production while the semantic fluency performance was not 2 SDs below typical mean. By contrast, her performance on phonological fluency and the written language tests was severely impaired.

Table 1. The AS patient performance on oral and written language measures

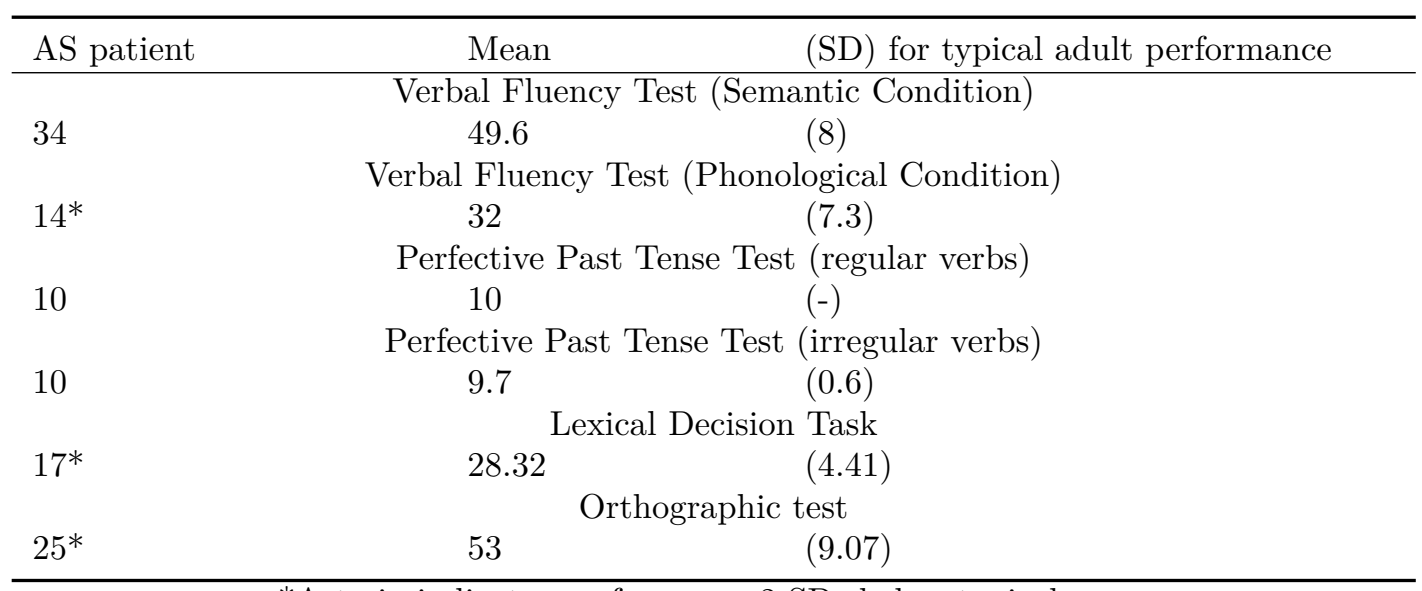

*Asterix indicates performance 2 SDs below typical mean.

\section{Discussion}

Our results indicate selective impairments in AS's linguistic performance. The patient showed severe limitations in phonological fluency and written language measures testing aspects of orthography in the presence of borderline performance on semantic fluency and totally typical performance on the morphological task (past tense production). Notably, while her spontaneous speech utterances were short, grammatical errors were not detected. Our results are in 
agreement with those findings showing limitations in the linguistic abilities of patients with AS (Jain \& Dinesh, 2016). However, these limitations appear to be highly selective in our patient. Furthermore, our data does not support the view that the corpus callosum is necessary for the typical development of specific language functions (for discussion see, Denis, 1981; Jeeves \& Temple, 1987) as our patient showed ceiling performance on past tense production.

\section{References}

Banerjee, T. K., Chattopadhyay, A., Manglik, A. K., \& Ghosh, B. (2006). Aicardi syndrome: a report of five Indian cases. Neurology India, 54(1), 91.

Barkovich, A. J., Kuzniecky, R. I., Jackson, G. D., Guerrini, R., \& Dobyns, W. B. (2005). A developmental and genetic classification for malformations of cortical development. Neurology, 65(12) 1873-1887.

Cabrera, M. T., Winn, B. J., Porco, T., Strominger, Z., Barkovich, A. J., Hoyt, C. S., ... \& Sherr, E. H. (2011). Laterality of brain and ocular lesions in Aicardi syndrome. Pediatric Neurology, 45(3), 149-154.

Dennis, M. (1981). Language in a congenitally acallosal brain. Brain and Language, 12(1), 33-53.

Grosso, S., Lasorella, G., Russo, A., Galluzzi, P., Morgese, G., \& Balestri, P. (2007). Aicardi syndrome with favorable outcome: case report and review. Brain and Development, $29(7), 443-446$.

Jain, S., \& Dinesh, N. (2016). A rare manifestation of specific learning disability in a child with Aicardi syndrome. Acta Oto-Laryngologica Case Reports, 1(1), 17-23.

Jeeves, M. A., \& Temple, C. M. (1987). A further study of language function in callosal agenesis. Brain and language, 32(2), 325-335.

Kosmidis, M.H., Vlahou, C.H., Panagiotaki, P., \& Kiosseoglou, G. (2004). The verbal fluency task in Greek population: Normative data, and clustering and switching strategies. Journal of The International Neuropsychological Society, 10, 164-172.

Lassonde, M., Bryden, M. P., \& Demers, P. (1990). The corpus callosum and cerebral speech lateralization. Brain and Language, 38(2), 195-206.

Lexical decision test. (2018-unpublished). Lab of Cognitive Neuroscience, School of Psychology, Aristotle University of Thessaloniki, Greece.

Lund, C., Bjørnvold, M., Tuft, M., Kostov, H., Røsby, O., \& Selmer, K. K. (2015). Aicardi syndrome: an epidemiologic and clinical study in Norway. Pediatric Neurology, 52(2), $182-186$.

Menezes, A. V., Enzenauer, R. W., \& Buncic, J. R. (1994). Aicardi syndrome-the elusive mild case. The British Journal of Ophthalmology, 78(6), 494.

Merrick, E. (2009). Case study: neuropsychologic functioning and complete agenesis of the corpus callosum. Archives of Clinical Neuropsychology, 24 (5), pp. 474-474). In Jain, S., \& Dinesh, N. (2016). A rare manifestation of specific learning disability in a child with Aicardi syndrome. Acta Oto-Laryngologica Case Reports, 1(1), 17-23.

Orthographic test for adolescents and adults. (2018-unpublished). Lab of Cognitive Neuroscience, School of Psychology, Aristotle University of Thessaloniki, Greece.

Prinzivalli, E., Quartini, A., Anastasia, A., Bersani, F. S., Colletti, C., Valeriani, G., \& Bersani, G. (2013). Compulsive behaviour in a patient with Aicardi syndrome. Agenesis of the corpus callosum. Journal of Psychopathology, 19, 359-362.

Rehmel, J. L., Brown, W. S., \& Paul, L. K. (2016). Proverb comprehension in individuals with agenesis of the corpus callosum. Brain and language, 160, 21-29.

Saddichha, S., Manjunatha, N., \& Akhtar, S. (2007). Typical Aicardi syndrome in a male. Acta Neuropsychiatrica, 19(5), 326-327.

Saini, L., Dekate, P. S., Prasad, V. V., \& Varma, D. R. (2018). Triad of gloom in a girl child: 
Aicardi syndrome. Neurology India, 66(1), 265.

Shirley, K., O'Keefe, M., McKee, S., \& McLoone, E. (2016). A clinical study of Aicardi syndrome in Northern Ireland: the spectrum of ophthalmic findings. Eye, 30(7), 1011.

Stavrakaki, S., \& Clahsen, H. (2009). The perfective past tense in Greek child language. Journal of child language, 36(1), 113-142.

Sutton, V. R., Hopkins, B. J., Eble, T. N., Gambhir, N., Lewis, R. A., \& Van den Veyver, I. B. (2005). Facial and physical features of Aicardi syndrome: infants to teenagers. American Journal of Medical Genetics Part A, 138(3), 254-258.

Tuft, M., Østby, Y., Nakken, K. O., \& Lund, C. (2017). Aicardi syndrome and cognitive abilities: A report of five cases. Epilepsy 85 Behavior, 73, 161-165.

Wechsler, D. (2008). Wechsler Adult Intelligence Scale-fourth edition (WAIS-IV). Standardization in Greek. Athens: Motivo. (2014).

Willis, J., \& Rosman, N. P. (1980). The Aicardi syndrome versus congenital infection: diagnostic considerations. The Journal of Pediatrics, 96(2), 235-239.

Zaarour, K. G., \& Traboulsi, E. I. (2017). Aicardi Syndrome (AIC). A Compendium of Inherited Disorders and the Eye, Oxford: University Press. 


\title{
Which word planning processes require attention? Evidence from dual-task interference in aphasia
}

\author{
Cyrielle Demierre $^{1}$; Grégoire Python ${ }^{1}$, Bertrand Glize $^{2}$, Laganaro Marina ${ }^{1}$ \\ ${ }^{1}$ Faculty of Psychology, University of Geneva, Switzerland \\ ${ }^{2}$ Physical and Rehabilitation Medicine Unit, EA4136, Bordeaux University Hospital
}

\begin{abstract}
Introduction
Although people can speak while doing another task (e.g. driving or cooking), it has been recognized that utterance planning is not entirely automatic and requires some amount of attention (Garrod \& Pickering, 2007; Roelofs, 2008). A growing literature uses dual-task paradigms to test attention requirements in word production as the cost of dual-task performance informs on the attentional requirement in each task (Ferreira \& Pashler, 2002). It has been shown in particular that conceptualization and lexical selection are under attentional demand (Roelofs \& Piai, 2011) and more recently, studies with healthy (Cook \& Meyer, 2008; Fargier \& Laganaro, 2019) and brain-damaged (aphasic) speakers (Laganaro, Bonnans \& Fargier, 2019) pointed to a role of attention in post-lexical processes. More precisely, interference on word production seems to increase by lengthening the stimulus onset asynchrony (SOA) in dual-tasks in healthy speakers (Fargier \& Laganaro, 2019) and an increase of phonological errors has been specifically reported in participants with impaired phonological encoding with concurrent stimuli appearing at SOA of $+300 \mathrm{~ms}$ (Laganaro, Bonnans \& Fargier, 2019). However, it is still unclear if different anomic profiles (e.g. semantic-lexical vs phonological) would respond specifically to different SOAs. If most word planning processes are under attentional control, interference should be maximal at early SOAs for participants with lexical-semantic impairment and at late SOAs for participants with phonological impairment.
\end{abstract}

\section{Methods}

Population

Data collection is currently ongoing with aphasic participants and healthy (non-brain damaged) controls. So far, three participants (aged 45-71, mean 56.5, three males), who suffer from aphasia following left hemispheric strokes, participated in the study. Two of them produced mainly semantic errors and the other one mainly phonological errors. Data collection is still underway to increase the sample size.

\section{Material and task}

Participants underwent a picture naming task and an auditory syllable detection task in isolation (single-task condition) and under dual-task condition. To avoid response strategies (e.g. waiting for the syllable to be played before naming the picture), single-task and dual-task trials were randomly presented. Auditory distractors were four different consonant-vowel syllables played at three SOAs $(+150 \mathrm{~ms},+300 \mathrm{~ms}$ or $+450 \mathrm{~ms})$. The participants were instructed to name the pictures as fast and accurately as possible, while pressing the space bar when they heard the syllable /fo/. Trials leading to a key press were discarded from the analyses (filler items).

\section{Results}

Preliminary analyses were carried out on naming latencies and on the amount and type of errors. Descriptively, the participant with a phonological profile produced more errors at late $\mathrm{SOA}(+450 \mathrm{~ms})$, whereas naming latencies were maximal with the mid SOA (+300 ms). In both patients with a lexical-semantic profile, errors and naming latencies increased for early SOAs (+150 and $+300 \mathrm{~ms})$. 


\section{Discussion}

The decrease of performance caused by the dual-task suggests early (lexical-semantic) interference in participants with lexical-semantic anomia and late (post-lexical) interference in the participant with phonological impairment. Therefore, limited attentional resources seem to impact lexical as well as post-lexical processes, but these preliminary results need to be confirmed with a larger group of participants.

\section{References}

Cook, A. E., \& Meyer, A. S. (2008). Capacity demands of phoneme selection in word production: New evidence from dual-task experiments. Journal of Experimental Psychology: Learning, Memory, and Cognition, 34(4), 886899.

Fargier, R. \& Laganaro, M. (2019). Interference in speaking while hearing and vice versa. Scientific Reports 9(1), 5375.

Ferreira, V. S., \& Pashler, H. (2002). Central bottleneck influences on the processing stages of word production. Journal of Experimental Psychology: Learning, Memory, and Cognition, 28(6), 11871199.

Garrod, S., \& Pickering, M. J. (2007). Automaticity of language production in monologue and dialogue. In A. Meyer, L. Wheeldon, \& A. Krott (Eds.), Automaticity and control in language processing (pp. 1-21). Hove: Psychology Press.

Laganaro, M, Bonnans, C. \& Fargier, R. (2019). Word form encoding is under attentional demand: evidence from dual-task interference in aphasia. Cognitive Neuropsychology.

Roelofs, A. (2008). Attention to Spoken Word Planning: Chronometric and Neuroimaging Evidence. Language and Linguistics Compass, 2(3), 389405.

Roelofs, A., \& Piai, V. (2011). Attention Demands of Spoken Word Planning: A Review. Frontiers in Psychology, 2. 


\title{
Contribution of acoustic analysis in the differential diagnosis of apraxia of speech
}

\author{
Bourqui Marion ${ }^{1}$; Pernon Michaela ${ }^{2,3}$, Fougeron Cécile ${ }^{2}$, Laganaro Marina ${ }^{1}$ \\ ${ }^{1}$ Faculty of Psychology, University of Geneva, Switzerland \\ ${ }^{2}$ Laboratoire de Phonétique et Phonologie, UMR7018, CNRS-Sorbonne Nouvelle, France \\ ${ }^{3}$ Department of Clinical Neurosciences, Geneva University Hospital, Switzerland
}

\section{Introduction}

Apraxia of speech (AoS) is commonly defined as a disorder of motor speech planning (phonetic encoding). It has also been identified as one of the first symptoms of neurodegenerative disorders such as cortico-basal degeneration or non-fluent primary progressive aphasia (GornoTempini et al., 2004).

The differential diagnosis of AoS has often been discussed in the literature, and may be confused with phonological disorder (impaired phonological encoding) or dysarthria (impaired motor execution).

Among the clinical signs of AoS, difficulties in coarticulation have been described (Ziegler \& von Cramon, 1986). One of the manifestations of such impaired coarticulation is represented by the insertion of schwas or transitional vocoid in consonants clusters (Duffy, 2012) and it has been shown that clusters are particularly difficult for AoS patients (Ziegler, W. \& Aichert, I., 2004).. However, such insertions are not always detectable by the human ear, in particular when they are very discrete. In the present study we investigate whether acoustic analyses can allow us to (1) detect the insertions of vocoids which are not perceived by the listener and (2) differentiate AoS from dysarthrias on this basis.

\section{Methods}

Participants

Nine participants who suffered from apraxia of speech following a left hemispheric stroke, four participants with primary progressive apraxia of speech, ten patients with hypokinetic dysarthria (Parkinson's disease), ten with mixed dysarthria (amyotrophic lateral sclerosis), ten with mixed-dystonic dysarthria (Wilson's disease) and forty control subjects with no history of neurological impairment participated in this study. All the participants were French-speaking.

\section{Material and Procedure}

All participants were evaluated with the MonPaGe speech assessment protocol (Fougeron et al., 2018), which evaluates different speech dimensions in various speech tasks. Among them a reading and/or repetition task whith a set of 52 pseudowords is used to assess the articulation of all French phonemes, with manipulation of position and syllable complexity in terms of structure and frequency.

\section{Results}

Participants productions were rated by two independent raters following the MonPaGe rating protocole. A directed perceptual evaluation of the production is made by ear according to the following dimensions (with yes or no answers): accuracy, insertion/inversion/omission of a segment, type of feature altered if any (place, manner, voicing, nasality, rounding), and effortful production.

Examination of the transition between consonants in the fourteen pseudowords with unvoiced 
cluster was then done with Praat on the visualization of the signal and spectrogram representations. Criteria for determining the presence of a transitional vocoid included: the presence of periodic vibration in the signal, the presence of formants in the mid frequency region (F1/F2 region), and/or an increase in energy which could be attributed to an increase in sonority or periodic event between the voiceless consonants in the cluster.

The analyses are ongoing. So far, insertions of vocalic elements are found in some, but not all, AoS patients and in none of the dysarthric or control participant. It is particularly interesting to note that some of these insertions are not detected at the perceptual level, but are visible on the spectrogram.

\section{Discussion}

Our preliminary results indicate that only participants with AoS insert vocalic elements in unvoiced clusters and that some of these insertions are only detected with acoustic analyses (are not perceived by the human ear). This result is in line with the literature showing specific acoustic features in AoS (Marczyk et al., 2019), and here we show that they differentiate AoS from 4 other groups of participants with different motor speech disorders. The observed vocalic insertions can have two different interpretations: (1) some patients may insert vowel elements into unvoiced clusters in order to facilitate the articulation of the two successive consonantal elements. This could be seen as a compensatory mechanism; or (2) these results could may also be consistent with previous studies mentioning coarticulation/coordination difficulties in patients with AoS. In fact, even those patients who do not insert still present specific spectrographic characteristics that show their difficulties difficulties of coordinating successive speech gestures in a fluid manner, which will be further analysed.

\section{References}

Duffy, J. (2012). Motor Speech Disorders: Substrates, Differential Diagnosis, and Management (3rd ed). St Louis, United States ; Elsevier.

Fougeron, C., Delvaux, L., Ménard, L. \& Laganaro, M. (2018, may). The MonPaGe_HA Database for the documentation of spoken French throughout adulthood. In Proc. 11th International Conference on Language Resources and Evaluation, Miyazaki, Japan.

Gorno-Tempini M. L., Dronkers N. F., Rankin K. P., Ogar J. M., Phengrasamy L., Rosen H. J. \& Miller B. L. (2004). Cognition and anatomy in three variants of primary progressive aphasia. Annals of Neurology, 55, 335-346.

Marczyk, A., Meynadier, Y., Gaydina, Y., Solé, M. J. (2019). Dynamic acoustic evidence of nasalization as a compensatory mechanism for voicing in spanish apraxic speech.

Ziegler, W. \& von Cramon, D. (1986). Disturbed Coarticulation in Apraxia of Speech: Acoustic Evidence. Brain and language, 29, 34-47.

Ziegler, W., Aichert, I. \& Staiger, A. (2012). Apraxia of Speech: Concepts and Controversies. Journal of speech, language and hearing research, 55, 1485-1501.

Ziegler, W. \& Aichert, I. (2004). Syllable frequency and syllable structure in apraxia of speech. Brain and Language, 88, 148-159. 


\title{
The investigation of paraphasias in speakers with fluent and non-fluent aphasia
}

\author{
Mile Vuković \& Irena Vuković \\ University of Belgrade - Faculty of Special Education and Rehabilitation, Serbia \\ Veljko Ramadanovic School for Visually Impaired Students, Zemun-Belgrade, Serbia
}

\section{Introduction}

A paraphasia is a symptom of aphasia in which an intended or targeted word is substituted for an incorrect word. Praphasias are produced unintentionally and are more typical for patients whose speech is uttered fluently. The major types of paraphasia are the phonemic, verbal, and neologistic. This study examines the occurrence and types of paraphasias in speakers with fluent and non-fluent aphasia. In addition, we wanted to explore the influence of language tasks on occurrence of paraphasia.

\section{Methods}

A total of 30 patients (15 with fluent and 15 with nonfluent aphasia), aged 55-73 participated in this study. All patients were right-handed, with a single left hemisphere CVA. The diagnsois and type of aphasia was done by Boston Diagnostic Aphasia Examination (Goodglas and Kaplan, 1983). For the analysis of paraphasia the following subtest from the BDAE were used: The Cookie theft picture, Repetition of words, Responsive naming, Visual Confrontation naming, as well as Boston naming test (Kaplan et al., 1983).

\section{Results}

The resuls have showen that both group of patients produce paraphasic errorrs during all kind of language tasks. Howevere, paraphasias are significatly more appeared in patients with fluent aphasia than in patients with nonfluent aphasia. Both group of speakers (fluent and non-fluent) had more phonemic praphasias than verbal and neologistic. Language task has significant impact on the frequency of paraphasias: the paraphasias were more appeared during confrontation naming and repetition than during expository speech.

\section{Discussion}

We could hypothesize that an impairment in lexical and phonological processing contributes to the occurrence of paraphasia in fluent and in nonfluent aphasia. The analysis of paraphasia in aphasic patients can provide additional information in understanding the nature of word production deficits and can assist in patient management.

\section{Acknowledgment}

The Ministry of Education, Science and Technological Development of Republic of Serbia has financially supported this work (Project No 179068).

\section{References}

Laganaro, M. \& Zimmermann, C. (2010). Origin of phoneme substitution and phoneme movement errors in aphasia. Language and Cognitive Processes, 25, 1, 1-37.

Schwartz, F.M., Dell, S.G., Martin, N., Gahl, S., Sobel, P. (2006). A case-series test of the interactive two-step model of lexical access: Evidence from picture naming. Journal of Memory and Language 54, 228-264.

Schwartz, F. M. (2015). Theoretical analysis of word production deficits in adult aphasia. Phil. Trans. R. Soc. B 369: 20120390. http://dx.doi.org/10.1098/rstb.2012.0390. 


\title{
Positive effects of a treatment based on temporal information processing on language and non-language cognitive functions in individuals with aphasia: a pilot study
}

\author{
Mateusz Choiński, Elżbieta Szeląg, Anna Bombińska, Aneta Szymaszek \\ Laboratory of Neuropsychology, Nencki Institute of Experimental Biology, Warsaw, Poland
}

\section{Introduction}

Temporal information processing (TIP) underlies many cognitive functions. Several studies indicated that TIP deficits are associated with deficits in processing of single units of language -phonemes (e.g. stop-consonants which are limited in time up to some tens of miliseconds). Several studies have shown that patients with left-hemispheric brain lesions and aphasia display deficient TIP on different time levels (millisecond or multisecond one), depending on aphasic symptoms they present. In patients with Wernicke's aphasia disturbed TIP on millisecond level was observed and reflected in a declined ability of sequential processing and temporal ordering. These deficits were evidenced in elevated thresholds for identification of events presented in rapid succession and observed in significantly longer, as compared to healthy controls, interstimulus interval (ISI) between two sounds, necessary to report correctly their temporal order (e.g., Swisher and Hirsh, 1972; Wittmann et al., 2004; Fink et al., 2006; Szelag et al. 2014, 2015). It resulted in deficient phonological awareness underlying receptive language deficits, thus, such ability is crucial for auditory comprehension (v. Steinbüchel et al., 1999; Wittmann at al., 2004, Sidiropoulos et al., 2010).

Traditional therapy of aphasia is based on speech and comprehension exercises used to improve deficient language functions directly. It was evidenced that aphasia is usually accompanied by deficits of non-language cognitive functions, i.e., executive functions, attention or working memory. Deficits in these cognitive functions do intensify the speech difficulties in aphasic subjects and thus, hinder the process of language restitution. Therefore, aphasiologists developed different training methods to enhance deficient non-language cognitive functions. Several studies have reported beneficial effects of working memory and attention trainings on sentence comprehension in individuals with aphasia (Javadipour et al., 2018; Majerus, 2018; Zakarias et al., 2018).

In our Laboratory of Neuropsychology, Nencki Institute of Experimental Biology PAS, the innovative therapeutic tool Dr. Neuronowski ${ }^{\circledR}$ based on TIP was developed. The majority of exercises included into this software involve millisecond TIP and sequencing abilities. The software was also extended by the training dedicated to improve other cognitive functions: attention, planning and auditory short-term memory.

The aim of the present study was to compare the effects of two therapy programs: a traditional language therapy and the novel treatment method using the software Dr. Neuronowski®

\section{Methods}

Subjects

Eight patients (6 male and 2 female) suffering from post-stroke, mixed aphasia after haemorrhage or infarction (lesion age $\mathrm{M}=8,5$ months; $\pm \mathrm{SD}=6.12$ months) participated in the study. They aged from 30 to 82 years ( $\mathrm{M}=63.75$ years; $\pm \mathrm{SD}= \pm 15.86$ years), were right-handed, Polish native speakers and had normal hearing level. Apart from stroke they had neither neurological nor psychiatric disorders and reported no history of head injuries in the past.

The damaged brain structures in both groups comprised mainly temporal and temporo-parietal areas of the left hemisphere which covered the classical regions involved in both receptive language function (auditory comprehension) and TIP (v. Steinbüchel et al., 1999; Wittmann et al., 2004; Szelag et al., 2008). 


\section{Procedure}

The study combined both cognitive evaluation procedures and training programs. Evaluation procedures were perfomed twice: before and after the training. Several cognitive functions were assessed. It included language functions: phoneme discrimination, word and sentence comprehension, as well as non-language cognitive functions: TIP, verbal and spatial shortterm memory, working memory, as well as planning.

Aphasic subjects were randomly assigned to one of two training group. They trained either with Dr. Neuronowski® software $(\mathrm{n}=4$; experimental group) or with the control training which combined classical speech therapy games $(n=4$; control group). The protocol in each training program consisted of 24 sessions performed three times a week.

\section{Results}

The results are displayed in Figure 1. Due to small number of subjects no statistical analyses were performed. In general, following Dr. Neuronowski ${ }^{\circledR}$ training amelioration of both trained non-language cognitive functions and untrained language functions was observed. The control training was mainly beneficial for trained language functions.

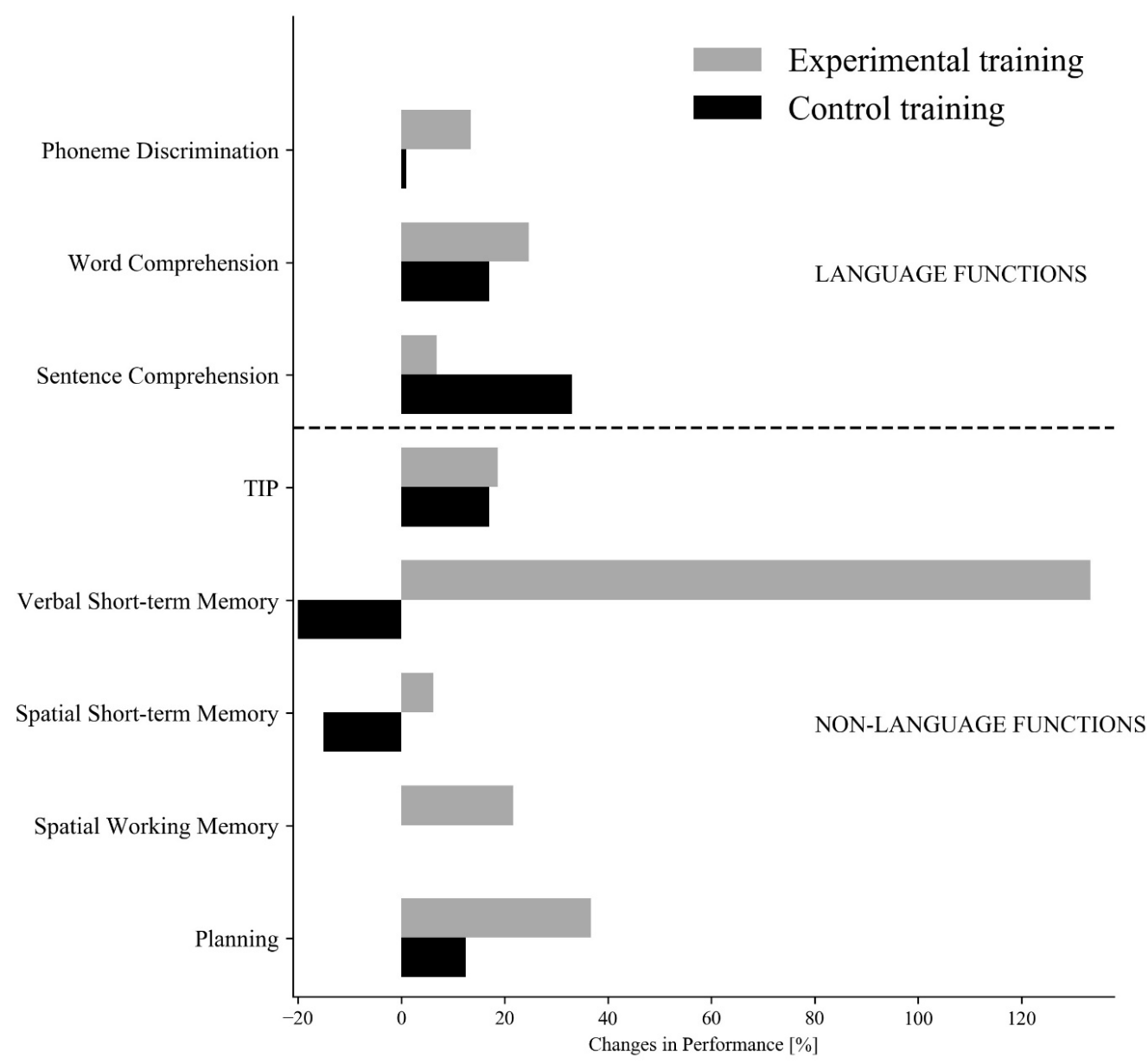

Figure 1. Percentage differences between the baseline and post-treatment performance (baseline -posttreatment/baseline* $100 \%$ ) for particular tasks for experimental group (grey bars) and control group (black bars). The 0 point reflects stable performance (no difference between baseline vs post-treatment performance). Positive values (right side from the 0 point) correspond to improved performance. Negative values correspond to worsened performance (left side from the 0 point) 


\section{Discussion}

The preliminary results indicated the divergent effects of both trainings. The control training was beneficial mainly for practiced language functions, however the slight amelioration in some untrained functions was also noted. On the other hand, the patients in experimental group improved both in terms of trained non-language cognitive functions including TIP and untrained language functions. In this group, the enhancement of unpracticed word and sentence comprehension may indicate the far transfer following the treatment with the Dr. Neuronowski ${ }^{\circledR}$ software. These results are in line with previous works reporting the close relationship between comprehension and TIP (Szeląg et al, 2015; Szymaszek et al., 2017).

On the basis of these preliminary results one may conclude that the application of training in TIP is a promising direction in aphasia rehabilitation. In the future we plan to increase the testing sample to verify the preliminary results reported here, as well as to perform the analysis of acquired EEG, fMRI and DTI data to explore the neural correlates of training-induced changes from the perspective of underlying neuroanatomical basis.

\section{Funding}

The research was supported by National Science Centre, Poland, grant number: 2016/21/B/ HS6/03775.

\section{References}

Fink M., Churan J. \& Wittmann M. (2006). Temporal processing and context dependency of phoneme discrimination in patients with aphasia. Brain Lang. 98(1), 1-11.

Javadipour, S., Nejati, V., Yadegari, F., Javadipour, S., \& Fazele, S. A. A. (2018). A Visual Attention Training Program for the Improvement of Auditory Comprehension in Aphasia. NeuroQuantology, 16(1).

Majerus, S. (2018). Working memory treatment in aphasia: A theoretical and quantitative review. Journal of Neurolinguistics, 48, 157-175.

Sidiropoulos K., Ackermann H., Wannke M. \& Hertrich, I. (2010). Temporal processing capabilities in repetition conduction aphasia. Brain Cogn, 73, 194-202.

Swisher L \& Hirsh IJ. (1972) Brain damage and the ordering of two temporally successive stimuli. Neuropsychologia, 10, 137-152.

Szelag E., Lewandowska M., Wolak T., Seniow J., Poniatowska R., Pöppel E. \& Szymaszek, A. (2014). Training in rapid auditory processing ameliorates auditory comprehension in aphasic patients: A randomized controlled pilot study. J Neurol Sci, 338(1), 77-86.

Szelag E., Dacewicz A., Szymaszek A., Wolak T., Senderski A., Domitrz I. \& Oron A. (2015). The application of timing in therapy of children and adults with language disorders. Front Psychol, 6, 1714.

Szymaszek A., Wolak T. \& Szelag E. (2017). The treatment based on temporal information processing reduces speech comprehension deficits in aphasic subjects. Front Aging Neurosci, 9, 98.

v. Steinbüchel N., Wittmann M., Strasburger H. \& Szelag E. (1999). Auditory temporalorder judgement is impaired in patients with cortical lesions in posterior regions of the left hemisphere. Neurosci Lett, 264(1-3), 168-171.

Wittmann, M., Burtscher, A., Fries, W. \& von Steinbüchel, N. (2004). Effects of brain-lesion size and location on temporal-order judgment. Neuroreport, 15(15), 2401-2405.

Zakariás L., Salis C. \& Wartenburger I. (2018). Transfer effects on spoken sentence comprehension and functional communication after working memory training in stroke aphasia. $J$ Neurolinguist, 48, 47-63. 


\title{
The production of French consonant sequences in typically developing children and in people with aphasia
}

\author{
Faith Chiu ${ }^{1}$, Typhanie Prince ${ }^{2}$ \\ ${ }^{1}$ Department of Psychology, University of York \\ ${ }^{2}$ University Training Center for Speech-Language Pathology, University of Nantes
}

\begin{abstract}
Introduction
All models of language production postulates that speakers generate phonological forms of words out of sublexical components as opposed to retrieving them as units from the mental lexicon (Meyer \& Belke, 2008). Phonological forms differentiate segments from metrical structure, and models vary in which the way segmental material is linked to metrical structure and whether activation of segmental material is parallel in the syllable (e.g., Dell, 1986 compared to Levelt et al., 1999) Our study considers if speech errors made by children during acquisition are mirrored by those made by people with aphasia (PwA). We focus in particular on consonant sequences, which have been shown to involve an interplay between segmental material and metrical syllabic structure.
\end{abstract}

\section{Methods}

\section{Participants}

Twenty monolingual French typically developing children $\left(\mathrm{M}_{\text {age }}=2 ; 34,8\right.$ female $)$ and twenty monolingual French people with vascular aphasia participated in this study $\left(\mathrm{M}_{\text {age }}=64 ; 10,11\right.$ female). None of the children's parents reported their children to have any speech and hearing deficits, nor any neurological or motor control disorders. All but one PwA suffered from a left hemisphere stroke; the exception had a right cerebrovascular accident. Parts of standardised batteries (HDAE, Mazaux \& Orgogozo, 1982 and MT-86, Nespoulous \& al. 1986-1992) were administered by a speech therapist to PwA to obtain their cognitive and linguistic profiles; only those with phonological deficits were selected for the study. All but two PwA participated in the experiment 2 to 25 days post-stroke; the other two completed the task during speech therapy. The breakdown of aphasia types by lesion were as follows: those resembling Broca's aphasia with an anterior damage $(\mathrm{n}=7$, where 4 of which also suffered from apraxia of speech), those with Wernicke's aphasia $(\mathrm{n}=6)$, conduction aphasia $(\mathrm{n}=4)$, and sensorial transcortical aphasia $(\mathrm{n}=3)$. All participants were based in Nantes: children were tested in their local kindergarten; PwA participated in the task in the hospital Neurovascular unit. Another group of native French speakers were also tested as controls $(\mathrm{n}=20)$.

\section{Material and Procedure}

PwA and children completed a picture-naming task containing a total of forty tokens which were bisyllabic or trisyllabic and contained consonant sequences at the beginning of the word (e.g. stylo 'pen'), or the middle (casquette 'cap') or the end (masque 'mask', veste 'jacket'). Participants were prompted by the experimenter when they were unable to retrieve a word from memory, after which they repeated the token. In this conference paper, only speech error data obtained by means of spontaneous production were analysed (i.e. without any repetition prompt). All utterances were transcribed by a trained French phonetician on PHON (Rose $\&$ al., 2006), and coded for transformations made to consonant sequences in the target token. These transformations could, for example, take the form of omitting the first or second of two consonants (deletion), and/or inserting a vowel to break up the two adjacent consonants in the sequence (epenthesis), and/or replacing one or both consonants with another consonant (substitution). 


\section{Results}

In spontaneous productions, both children and PwA produced speech errors on consonant sequences. However, these errors were not identical in nature of transformation. Where tokens were obtained in spontaneous production, it was largely only PwA who transformed word-initial consonant sequences. PwA with and without apraxia produced initial sequences differently: deletion of the first consonant dominated speech errors by those with apraxia, while other transformation options were observed for PwA without apraxia. It was only children who mostly struggled with consonant sequences in the middle of word; speech errors for medial sequences involved transformations via deletion of either the first or both consonants in the sequence. All groups made transformations to word-final sequences via a mixture of transformation methods, predominantly deletion of either the first or second consonant and/or substitution.

\section{Discussion}

We interpret that transformations by $\mathrm{PwA}$ of consonant sequences at the beginning of the word to indicate complexity of such initial sequences in both motor planning and in phonological structure. The fact that PwA without apraxia also struggled with such initial sequences and transformed them differentially demonstrates that, even when motor planning difficulties were accounted for, such initial sequences also present difficulty at the level of phonological representation. Children's difficulty with medial sequences could imply something about the nature of these sequences which makes them difficult to acquire. Yet, once these medial sequences are mastered, they remain relatively stable in phonological representation since PwA made few speech errors with these.

Our study demonstrated differential treatment of phonologically complex consonant sequences in $\mathrm{PwA}$ and during language acquisition. The fact that consonant sequences are modified in both acquisition (in children) and loss (in PwA with and without apraxia of speech) indicates an underlying complexity resultant of metrical structure (i.e. the syllable). Differential modifications by both groups should help shed light on models of language production and the various levels within phonological encoding also for the healthy individual.

\section{References}

Dell, G. S. (1986) A spreading-activation theory of retrieval in sentence production. Psychological Review, 93: 283-321.

Levelt, W.JL, Roelofs, A. \& Meyer, A. (1999). A theory of lexical access in Speech production. Behavioral and Brain Sciences, 22: 1-75.

Meyer, A. \& Belke, E. (2008). Word form retrieval in language production, in M. G. Gaskell (ed.). The Oxford Handbook of Psycholinguistics. Oxford: Oxford University Press, 471-487.

Rose, Y., MacWhinney, B., Byrne, R., Hedlund, G., Maddocks, K. O’Brien, P. and Wareham, T. (2006) Introducing Phon: A software solution for the study of phonological acquisition, in Bamman, D, Magnitskaia, T, and Zaller, C, (eds.) Proceedings of the 30th annual Boston University Conference on Language Development. Somerville, MA: Cascadilla Press, 489-500. 


\title{
Grammatical-gender effects in noun-noun compound production: Evidence from aphasia
}

\author{
Antje Lorenz $^{1}$, Danièle Pino ${ }^{2,3}$, Jörg D. Jescheniak ${ }^{4}$, Frank Regenbrecht ${ }^{2}$, Hellmuth Obrig ${ }^{2,3}$ \\ ${ }^{1}$ Humboldt-Universität zu Berlin \\ ${ }^{2}$ University Hospital, Leipzig \\ ${ }^{3}$ Max Planck Institute for Human Cognitive and Brain Sciences, Leipzig \\ ${ }^{4}$ Leipzig University
}

\section{Introduction}

It is still a matter of debate if and how the morphological structure of complex words, such as compounds (e.g., goldfish), determines their lexical representation and processing. Especially for speech production, this topic is controversial. The present study examines how compounds are stored at a lexical-syntactic level, i.e. the lemma level (Levelt, Roelofs, \& Meyer, 1999). We test whether compounds are stored holistically ('single-lemma representation account', e.g., Levelt et al., 1999), or as multiple lemmas, with each lemma corresponding to a constituent morpheme of the target compound, in addition to a holistic compound lemma ('multiple-lemma representation account', see Marelli, Aggujaro, Molteni, \& Luzzatti, 2012). While empirical data from healthy adult speakers mostly support the notion of single, holistic compound lemmas (Lorenz, Mädebach, \& Jescheniak, 2018; Lorenz, Regel, Zwitserlood, \& Abdel Rahman, 2018; Lüttmann, Zwitserlood, Böhl, \& Bölte, 2011), data from participants with aphasia speak for representation of multiple lemmas in compounds (Marelli et al., 2012; Lorenz, Heide, \& Burchert, 2014). In the present (ongoing) study, participants with aphasia and word-finding difficulties were engaged in a primed picture-naming task with noun-noun compounds as targets. Effects of auditory definite determiner primes that are marked for grammatical gender

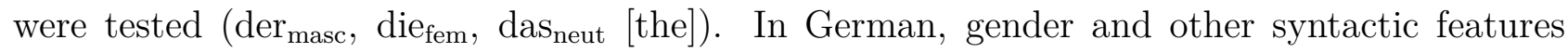
of morphologically complex words are determined by the rightmost constituent, i.e. the morphological head (e.g., Fisch masc [fish] in Goldfisch masc [goldfish]). Thus, gender of non-head constituents (e.g., Gold neut $_{\text {in }}$ Goldfisch $_{\text {masc }}$ ) is irrelevant for gender specification of the whole word. Our study examines whether gender of the first constituent (i.e., the modifier) of nounnoun compounds is nevertheless activated in compound production, which would point to a multiple-lemma representation. In this case, the modifier's gender should modulate the determiner priming effects in compound production.

\section{Methods}

\section{Participants}

Participants were recruited who suffer from mild to moderate word-finding difficulties in spontaneous speech and picture naming. Exclusion criteria were severe apraxia of speech or dysarthria, severe post-lexical phonological deficits and peripheral hearing deficits. Until today, 14 participants with aphasia or residual aphasia have been enrolled. We plan to include about 24 participants.

\section{Procedure}

All participants were tested individually in the Clinic for Cognitive Neurology at the University Hospital Leipzig. There were two or three testing sessions per participant, lasting for about $2.5 \mathrm{~h}$ each. Additional assessments included paper \& pencil tasks that examined the processing of gender, determiners and compound nouns in comprehension and production (Lorenz \& Zwitserlood, 2014). The main experiment was a primed picture-naming task with noun-noun compounds as targets. 54 color photographs of corresponding objects were used as experimental items. Grammatical gender of modifier and head always differed and system- 
atically varied. Each possible gender combination was used equally often. Each picture was presented three times throughout the experiment, once in combination with each of the three gender-marked determiners (der, die, das [the]). The following conditions were included: (1) determiner prime gender-congruent with the head/ compound (e.g., der masc $\rightarrow$ Gold $_{\text {neut }}$ fisch $_{\text {masc }}$ ), (2) determiner prime gender-congruent with the modifier (e.g., das neut $_{\text {) }}$, (3) determiner prime neither gender-congruent with the head nor the modifier (e.g., die $e_{f e m}$ ). Participants received a pseudo-randomized list, including 216 trials. To increase statistical power, testing was repeated once if tolerated. Before the start of the experiment, participants were familiarized with the target pictures and corresponding names. During the main experiment, a spoken determiner prime was presented over headphones starting $300 \mathrm{~ms}$ before presentation of the picture. Participants were instructed to name the pictures as quickly as possible with one word and to try to ignore the spoken determiners. Time out was set to 10 seconds. Both picture-naming accuracies and latencies were measured offline. All responses were recorded and transcribed, and speech onset latencies were determined using CheckFiles (Protopapas, 2006). Both group-level and single-case analyses were performed.

\section{Results}

Mean picture-naming accuracy of the preliminary group $(n=14)$ varied between 48 and $96 \%$ correct responses $(M=75 \%, s d=43$; first testing session). For the group, (generalized) linear mixed models confirmed faster and more accurate responses for head-congruent than incongruent determiner primes (RT analysis: $b=0.067, S E=0.018, t=3.734, p<0.001$; accuracy analysis: $b=-0.314, S E=0.131, z=-2.393, p=0.0167)$. No significant group effect was obtained for modifier-congruent primes $(z / t<1$; see Figure 1$)$. In contrast, single-case analyses revealed priming effects also for modifier-congruency in some participants (RT analysis: $\mathrm{n}=3$ $(p<0.05$ each); accuracy analysis: $\mathrm{n}=2(p<0.05)$, statistical trend: $\mathrm{n}=2(p<0.1))$. Both inhibitory and facilitatory effects of modifier-congruent primes were obtained. Furthermore, effects by head-congruent primes were confirmed for 6 participants in reaction times $(\mathrm{n}=5(p$ $<0.05), \mathrm{n}=1(p<0.1)$, and three participants in accuracies; $\mathrm{n}=2(p<0.05), \mathrm{n}=1(p<0.1))$.

\section{Discussion}

Overall, participants showed faster and more accurate picture naming with head-congruent than incongruent determiner primes. In contrast, effects of modifier-congruent primes were not significant for the group. This replicates findings in healthy speakers (Lorenz et al., 2018), and is in line with single, holistic compound lemmas (Levelt et al., 1999). However, single-case analyses confirmed head-congruency effects for a subgroup of participants, only. Furthermore, some participants showed modifier-congruency effects, suggesting that constituent-lemmas of compounds were activated during compound retrieval. To sum up, there is a high degree of variance in our data, which is likely to be related to different underlying functional deficits in our participants. The data presented here are based on the first testing session, exclusively, and, thus, must be judged preliminary. More detailed analyses and inclusion of cerebral lesion pattern are currently performed. The origin of determiner-priming effects in speech production will be discussed with regard to the underlying functional deficits of individual participants and their lesion pattern.

\section{References}

Levelt, W. J. M., Roelofs, A., \& Meyer, A. S. (1999). A theory of lexical access in speech production. Behavioral and Brain Sciences, 22(1), 1-38. https://doi.org/10.1017/S0140525X99001776

Lorenz, A., Heide, J., \& Burchert, F. (2014). Compound naming in aphasia: Effects of complexity, part of speech, and semantic transparency. Language, Cognition and Neuro- 


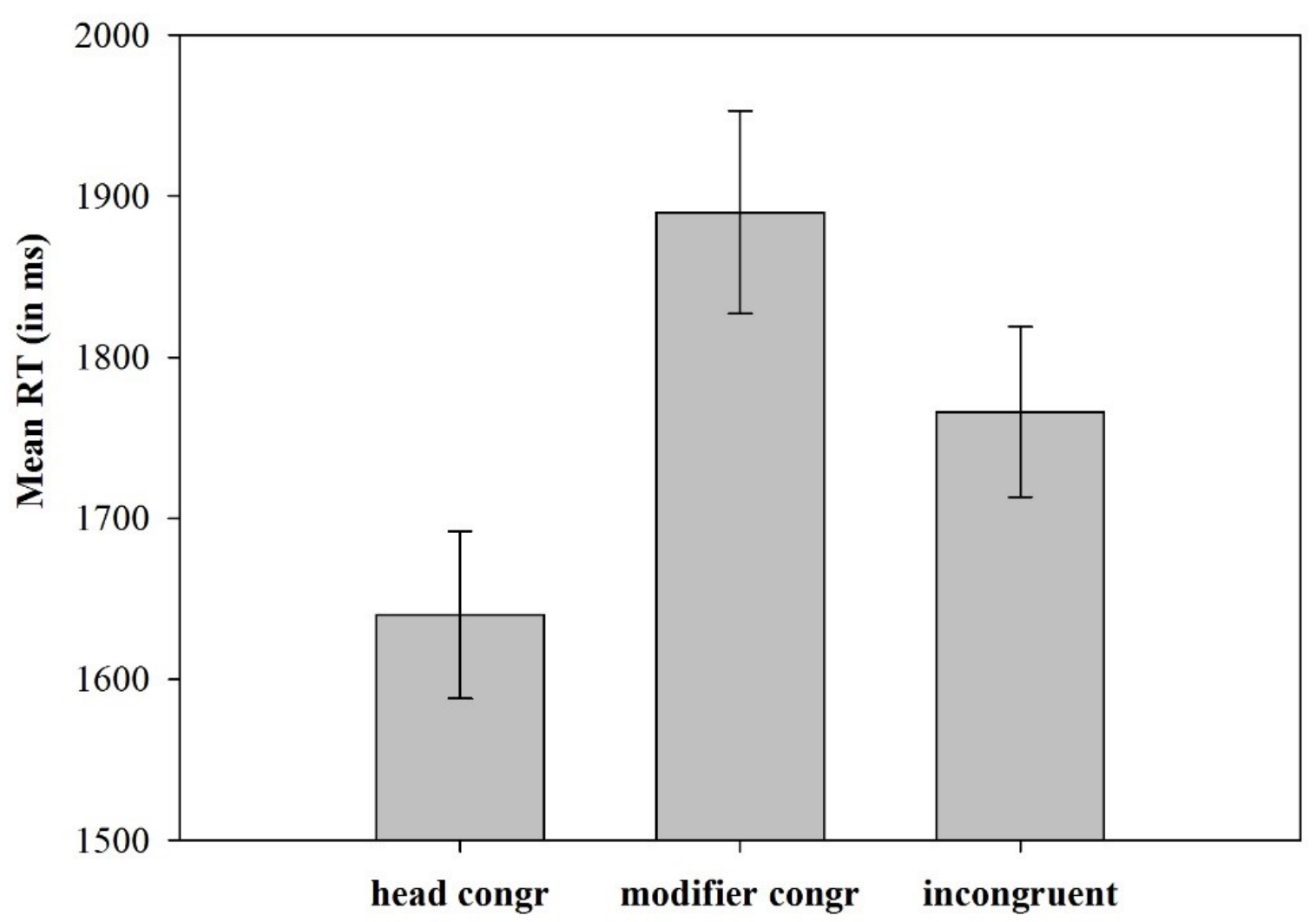

Figure 1. Compound naming with spoken determiner primes: Mean picture-naming latencies and standard errors of 14 participants. head congr: head-congruent determiner prime; modifier congr: modifier-congruent determiner prime; incongruent: head- and modifier-incongruent determiner prime

science, 29(1), 88-106. https://doi.org/10.1080/01690965.2013.766357

Lorenz, A., Mädebach, A., \& Jescheniak, J. D. (2018). Grammatical-gender effects in nounnoun compound production: Evidence from German. Quarterly Journal of Experimental Psychology (2006), 71(5), 1134-1149. https://doi.org/10.1080/17470218.2017.1310916

Lorenz, A., Regel, S., Zwitserlood, P., \& Abdel Rahman, R. (2018). Age-related effects in compound production: Intact lexical representations but more effortful encoding. Acta Psychologica, 191(October), 289-309. https://doi.org/10.1016/j.actpsy.2018.09.001

Lorenz, A., \& Zwitserlood, P. (2014). Processing of nominal compounds and gender-marked determiners in aphasia: Evidence from German. Cognitive Neuropsychology, 31(1-2), 4074. https://doi.org/10.1080/02643294.2013.874335

Marelli, M., Aggujaro, S., Molteni, F., \& Luzzatti, C. (2012). The multiple-lemma representation of Italian compound nouns: A single case study of deep dyslexia. Neuropsychologia, 50(5), 852-861. https://doi.org/10.1016/j.neuropsychologia.2012.01.021

Protopapas, A. (2006). CheckVocal: A program to facilitate checking the accuracy and response time of vocal responses from DMDX. Behavior Research Methods, 39(September), 1-5. Retrieved from https://link.springer.com/article/10.3758/BF03192979 


\title{
Investigating the Neural Correlates of Argument Structure Processing
}

\author{
Ekaterina Delikishkina ${ }^{1,4}$, Angelika Lingnau ${ }^{1,2,3}$, Gabriele Miceli ${ }^{1,2,5}$ \\ ${ }^{1}$ Center for Mind/Brain Sciences (CIMeC), University of Trento, Italy \\ ${ }^{2}$ Department of Cognitive Sciences, University of Trento, Italy \\ ${ }^{3}$ Institute of Psychology, University of Regensburg, Germany \\ ${ }^{4}$ International Doctorate for Experimental Approaches to Language and Brain (IDEALAB), \\ Universities of Trento, Groningen, Potsdam, Newcastle and Macquarie University \\ ${ }^{5}$ Centro Linceo Interdisciplinare 'Beniamino Segre', Accademia dei Lincei, Rome, Italy
}

Argument structure is one of the key aspects of verb processing. It encodes the core participants of an action - the actor himself and, if the action has a goal, the undergoer. It represents an important aspect of verb semantics and, at the same time, encodes the syntactic frame on which the sentence is built. We examined the neural correlates of argument structure processing by comparing transitive (i.e., two-argument) and intransitive (i.e., one-argument) verbs. Based on the previous reports (for review, see Thompson \& Meltzer-Asscher, 2014), we anticipated that greater argument structure complexity would be associated with increased recruitment of the temporal, parietal and prefrontal areas.

\section{Methods}

\section{Participants, Stimuli and Task}

Twenty healthy native Italian speakers were presented with line drawings of transitive (e.g., waving a flag) and intransitive (e.g., skiing) actions in a 3 Tesla MRI scanner. Participants were instructed to name depicted actions using a single verb in the infinitive form. Stimuli were presented in a rapid event-related design in pseudorandom order.

\section{Data analysis}

For the first-level analysis, individual functional MRI data were projected onto the reconstructed cortical surfaces of the left and the right hemisphere. Trial-wise $t$-maps of the two experimental conditions were used to train a linear discriminant analysis classifier to decode transitive and intransitive trials in searchlights created with an $8-\mathrm{mm}$ radius across the cortex. Decoding accuracies were obtained using leave-one-out cross-validation and averaged across the folds. At the group level, individual accuracy maps were aligned to the group-averaged hemispheric meshes using cortex-based alignment and submitted to a one-sample $t$-test to identify vertices where accuracy was significantly above chance. The resulting maps were corrected at = .05 (two-tailed) using Threshold-Free Cluster Enhancement with 5000 Monte Carlo simulations.

\section{Results}

As predicted, transitive vs. intransitive trials could be decoded in several brain areas (Figure 1), including the entire stretch of the left intraparietal sulcus (IPS), the left pars opercularis (ParsOp)/precentral gyrus, as well as the lateral occipitotemporal cortex (LOTC) and the posterior fusiform bilaterally.

\section{Discussion}

Transitivity effects in the left ParsOp replicate the results obtained by den Ouden et al. (2009). Notably, greater recruitment of the ParsOp by verbs with complex argument structure was reported in word production (naming task in den Ouden et al., 2009), but not in comprehension (lexical decision tasks in Thompson et al. 2007, 2010), consistent with the possibility that this 


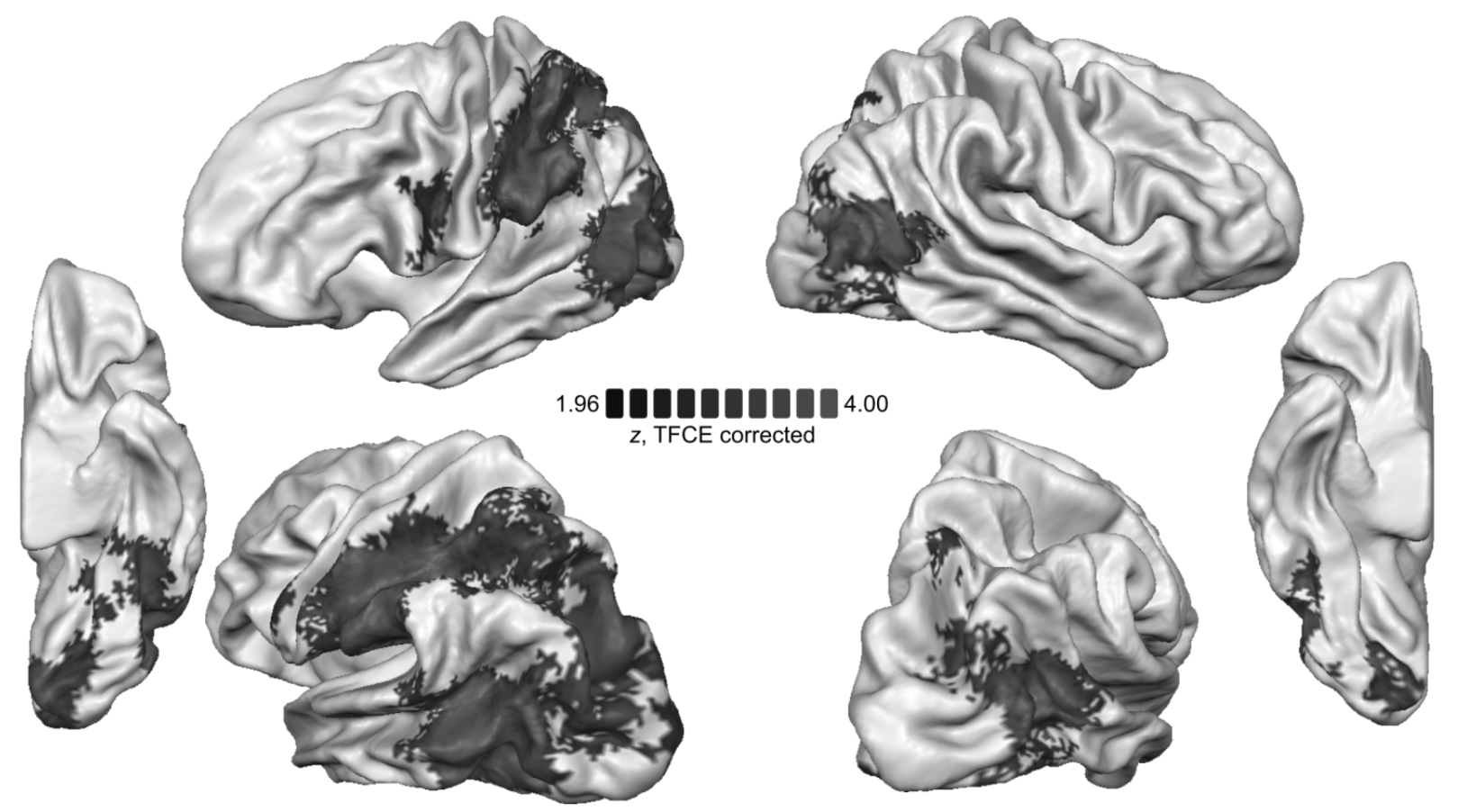

Figure 1. Multivariate decoding of transitive and intransitive verbs. Statistical group maps are showing vertices where average decoding accuracy was significantly above chance, corrected using ThresholdFree Cluster Enhancement at $=.05$ (two-tailed). The maps are projected onto the group-averaged surface meshes of the left and the right hemisphere.

area is involved in initial phrase structure building (Friederici, 2011).

Our IPS cluster overlaps with the cluster reported by den Ouden et al. (2009) and is in close vicinity to the angular/supramarginal gyrus identified by Thompson et al. (2007) as the area processing the number of arguments. On an alternative account, this activation may be associated with observation of goal-directed actions, which are transitive by definition (Culham \& Valyear, 2006).

Involvement of the LOTC in argument structure processing was previously reported during both verb comprehension (Thompson et al., 2007, 2010; Meltzer-Asscher et al., 2015) and verb production (den Ouden et al., 2009). Yet, transitivity effects in the LOTC may at least partially pertain to the nature of our stimuli, as this area is implied in tool viewing (Bracci et al., 2012). Transitive and intransitive trials could also be distinguished in the bilateral fusiform, which was expected, as object representations are believed to be stored in ventral temporal cortices (Martin, 2007).

Overall, the present results suggest that the argument structure is activated already at a singleword level. However, at least some of the observed effects may also be explained by perceptual and conceptual differences between the transitive and intransitive stimuli. Future studies should aim to tease these two accounts apart.

\section{References}

Bracci, S., Cavina-Pratesi, C., Ietswaart, M., Caramazza, A., \& Peelen, M. V. (2012). Closely overlapping responses to tools and hands in left lateral occipitotemporal cortex, Journal of Neurophysiology, 107(5), 1443-1456. 
Culham, J. C., \& Valyear, K. F. (2006). Human parietal cortex in action, Current Opinion in Neurobiology, 16(2), 205-212.

den Ouden, D. B., Fix, S., Parrish, T. B., \& Thompson, C. K. (2009). Argument structure effects in action verb naming in static and dynamic conditions, Journal of Neurolinguistics, 22(2), 196-215.

Friederici, A. D. (2011). The brain basis of language processing: From structure to function, Physiological Reviews, 91(4), 1357-1392.

Martin, A. (2007). The representation of object concepts in the brain, Annual Review of Psychology, 58, 25-45.

Meltzer-Asscher, A., Mack, J. E., Barbieri, E., \& Thompson, C. K. (2015). How the brain processes different dimensions of argument structure complexity: Evidence from fMRI, Brain and Language, 142, 65-75.

Thompson, C.K. \& Meltzer-Asscher, A. (2014), Neurocognitive mechanisms of verb argument structure processing, In Structuring the argument: Multidisciplinary research on verb argument structure, 141-168.

Thompson, C. K., Bonakdarpour, B., \& Fix, S. F. (2010). Neural mechanisms of verb argument structure processing in agrammatic aphasic and healthy age-matched listeners, Journal of Cognitive Neuroscience, 22(9), 1993-2011.

Thompson, C. K., Bonakdarpour, B., Fix, S. C., Blumenfeld, H. K., Parrish, T. B., Gitelman, D. R., \& Mesulam, M. M. (2007). Neural correlates of verb argument structure processing, Journal of Cognitive Neuroscience, 19(11), 1753-1767. 
Oral Presentations III 


\title{
Impaired verb-related morphosyntactic production in Multiple Sclerosis: Evidence from Greek
}

\author{
Valantis Fyndanis ${ }^{1}$, Lambros Messinis $^{2}$, Grigorios Nasios $^{3}$, Efthimios Dardiotis ${ }^{4}$, Maria \\ Martzoukou $^{3}$, Maria Pitopoulou ${ }^{3}$, Katerina Ntoskou ${ }^{5}$, Sonia Malefaki ${ }^{6}$ \\ ${ }^{1}$ Department of Communication Sciences and Disorders, Long Island University, Brooklyn \\ Campus, USA \\ ${ }^{2}$ Neuropsychology Section, Departments of Psychiatry and Neurology, University Hospital of \\ Patras and University of Patras Medical School, Patras, Greece \\ ${ }^{3}$ Department of Speech and Language Therapy, School of Health Sciences, University of \\ Ioannina, Ioannina, Greece \\ ${ }^{4}$ Department of Neurology, University Hospital of Larisa, University of Thessaly, Larisa, \\ Greece \\ ${ }^{5}$ Rehabilitation Unit for Patients with Spinal Cord Injury, "Demetrios and Vera Sfikas", \\ Department of Medicine, University of Patras, Patras, Greece \\ ${ }^{6}$ Department of Mechanical Engineering and Aeronautics, University of Patras, Patras, Greece
}

\section{Introduction}

Multiple Sclerosis (MS) is a neurodegenerative disease that predominantly affects sensorimotor and physical abilities. Cognitive deficits affecting working memory (WM), long-term memory, executive functioning, attention, processing speed, and visuospatial perception are also common in MS (e.g., Brochet \& Ruet, 2019; Rao, 1995), with deficits at least in WM and processing speed being more frequent and severe in Secondary Progressive MS (SPMS) than in RelapsingRemitting MS (RRMS) (Brochet \& Ruet, 2019, and references therein). To date, the evidence regarding language abilities in MS is inconclusive. A recent systematic review (Renauld et al., 2016) showed that "language impairments are possible but not very common in MS patients" (p. 110). Moreover, there are significant gaps in our knowledge about language abilities in MS. Morphosyntactic production, for example, has not been explored adequately thus far. This study investigates verb-related morphosyntactic abilities in MS focusing on Greek, a highly inflected language.

Recent evidence suggests that, contrary to what was long believed, verb-related morphosyntactic production can be selectively impaired in neurodegenerative disorders such as Alzheimer's disease (AD) (e.g., Fyndanis et al., 2013; 2018b). For instance, Fyndanis et al. (2013) found that, in Greek AD, Aspect is more impaired than Time Reference/Tense and subject-verb Agreement, and Time Reference is more impaired than Agreement. Interestingly, the same pattern emerged in Greek aphasia and healthy aging (e.g., Fyndanis et al., 2012; 2018a). That the same pattern emerged not only in AD and aphasia but also in healthy speakers (e.g., Fyndanis et al., 2018a) is consistent with the idea that pathology only exacerbates patterns or trends observed in healthy speakers (e.g., Dick et al., 2001; Miyake et al., 1994). Furthermore, recent studies showed that WM is critically involved in verb-related morphosyntactic production (e.g., Fyndanis et al., 2018a; Kok et al., 2007).

Given that MS is characterized by WM limitations (Brochet \& Ruet, 2019) —among other deficits-, we expect individuals with MS to perform worse than healthy controls on tasks tapping into morphosyntactic production. We also expect them to exhibit a pattern of performance identical or similar to that emerged in Greek AD (Fyndanis et al., 2013), aphasia (Fyndanis et al., 2012; 2018a) and healthy aging (Fyndanis et al., 2018a) (i.e., Aspect<Time Reference $<$ Agreement). Moreover, since individuals with SPMS have greater cognitive/WM limitations than individuals with RRMS (Brochet \& Ruet, 2019), we expect the former to be more impaired than the latter in morphosyntactic production. 


\section{Methods}

\section{Participants}

Thirty-eight individuals with MS took part in the study. There were 25 participants with RRMS (mean age=43.1, $\mathrm{SD}=8.4$; mean (number of years of formal) education=13.8; $\mathrm{SD}=3.9$ ) and 13 participants with SPMS (mean age=50.8, $\mathrm{SD}=9.7$; mean education=12.2; $\mathrm{SD}=3.8$ ). The RRMS and SPMS groups were matched with control group 1 and control group 2, respectively, on age, education, and gender.

\section{Experiment}

Participants were administered a sentence completion task (developed by Fyndanis et al., 2018a) tapping into the production of subject-verb Agreement, Time Reference/Tense, and Aspect. The task consists of 192 experimental items: 64 items tapped into Agreement, 64 Time Reference, and 64 Aspect. There were two lists. Each list consisted of 96 items: 32 items tested Agreement, 32 items tested Time Reference, and 32 items tested Aspect. Each participant with MS was administered one list only. Most healthy participants were administered both lists. Participants heard a source sentence (SS) and the beginning of the target sentence (TS). They were required to complete the TS by providing the missing verb phrase. Participants always had to produce a different form of the verb that appeared in the SS.

\section{Results}

Overall, the two MS groups outperformed the corresponding control groups (since the normality assumption was not met, Mann-Whitney $\mathrm{U}$ test was utilized; $p<0.001$ ). The RRMS group fared significantly worse than control group 1 on all three morphosyntactic conditions (MannWhitney U test: Agreement, $p=0.031$; Time Reference, $p=0.035$; Aspect, $p=0.001$ ), whereas the SPMS group fared significantly worse than control group 2 on Aspect $(p=0.002)$ and Time Reference $(p=0.018)$. Within-group comparisons using related-samples Friedman's test and adjusting significance values by the Bonferroni correction for multiple pairwise post-hoc tests showed that all four groups exhibited the same pattern of performance: they performed significantly worse on Aspect than on Agreement (RRMS, $p=0.002$; Control Group 1, $p=0.015$; SPMS, $p=0.005$; Control Group 2, $p=0.010$ ) and Time Reference (RRMS, $p<0.001$; Control Group 1, $p=0.008$; SPMS, $p=0.001$; Control Group 2, $p=0.006$ ) (Figure 1). In all four groups, the difference between Agreement and Time Reference was not significant. The RRMS group did not differ significantly from the SPMS group in any of the three conditions. Likewise, the overall performances of the RRMS and SPMS groups did not differ significantly.

\section{Discussion}

The overall performance of the RRMS and SPMS groups was significantly worse than that of the control groups, showing that individuals with MS can be impaired in verb-related morphosyntactic production. All four groups performed worse on Aspect than on subject-verb Agreement and Time Reference. The two MS groups, thus, differed from their control groups in morphosyntactic production quantitatively but not qualitatively. This is consistent with the idea that pathology only exacerbates patterns or trends observed in healthy speakers (e.g., Dick et al., 2001; Miyake et al., 1994). What follows from this idea is that patterns of morphosyntactic production cannot be pathology-specific, meaning that the same or similar patterns should emerge in different neurological conditions. This prediction is largely borne out by the fact that the pattern reported here for MS is similar to the patterns reported for Greek aphasia (Fyndanis et al., 2012; Fyndanis et al., 2018a) and AD (Fyndanis et al., 2013) in that, in all three neurological conditions, Aspect was found to be more impaired than Agreement and Time Reference.

Contrary to our prediction, the RRMS group did not differ significantly from the SPMS group 


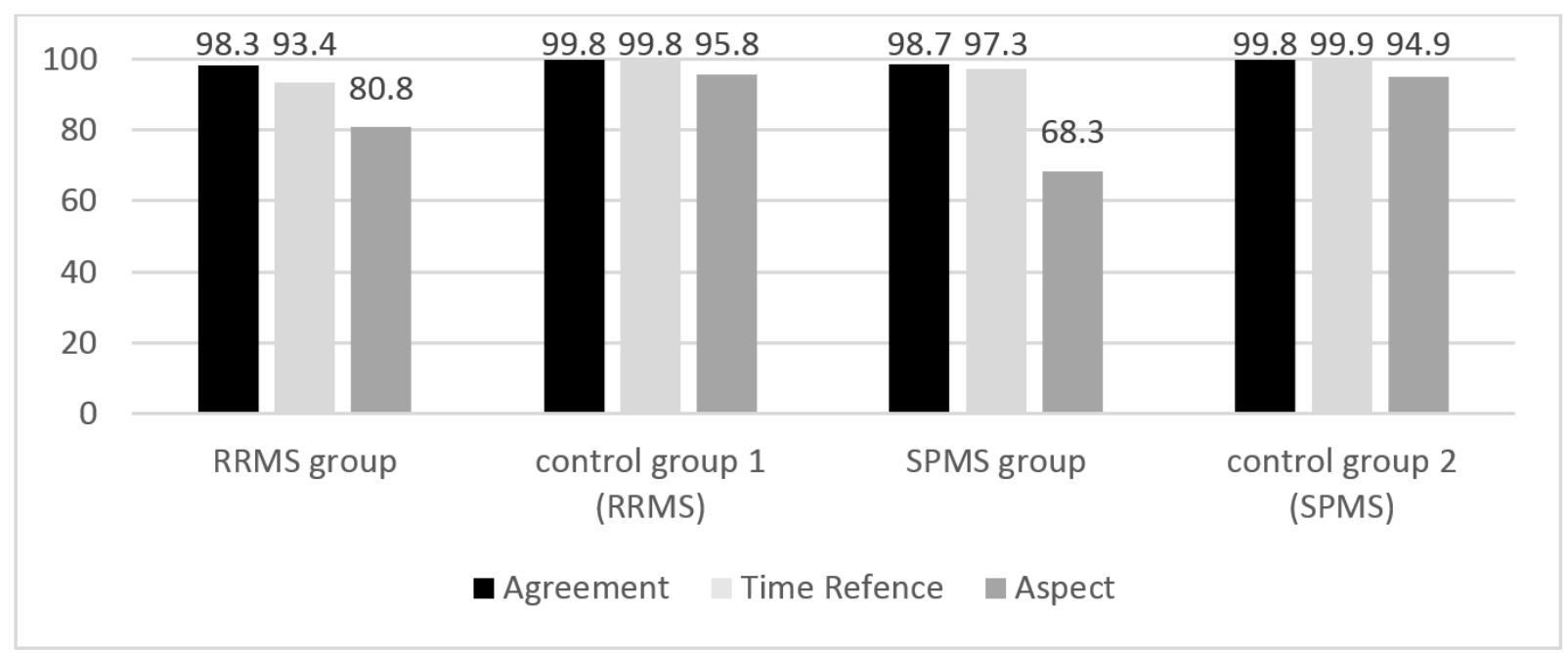

Figure 1. Accuracy (\%) of the four groups on the production of subject-verb Agreement, Time Reference, and Aspect.

in any of the three morphosyntactic conditions. The SPMS group, however, performed worse (albeit not significantly so) than the RRMS group on the most demanding morphosyntactic category, Aspect, which allows us to speculate that increasing the samples of RRMS and SPMS participants would result in significant between-MS subgroup differences in Aspect.

\section{References}

Brochet, B., \& Ruet, A. (2019). Cognitive impairment in multiple sclerosis with regards to disease duration and clinical phenotypes. Frontiers in Neurology, 10: 261. Doi: 10.3389/fneur.2019.00261

Dick, F., Bates, E., Wulfeck, B., Utman, J.A., Dronkers, N., \& Gernsbacher, M.A. (2001). Language deficits, localization, and grammar: Evidence for a distributive model of language breakdown in aphasic patients and neurologically intact individuals. Psychological Review, 108, 759-788. Doi: 10.I037//0033-295X.108.4.759

Fyndanis, V., Arcara, G., Christidou, P., \& Caplan, D. (2018a). Morphosyntactic production and verbal working memory: Evidence from Greek aphasia and healthy aging. Journal of Speech, Language and Hearing Research, 61, 1171-1187. Doi: 10.1044/2018_JSLHRL-17-0103

Fyndanis, V., Arfani, D., Varlokosta, S., Burgio, F., Maculan, A., Miceli, G., Arcara, G., Palla, F., Cagnin, A., Papageorgiou, S., \& Semenza, C. (2018b). Morphosyntactic production in Greek- and Italian-speaking individuals with probable Alzheimer's disease: Evidence from subject-verb agreement, tense/time reference, and mood. Aphasiology, 32, 61-87. Doi: $10.1080 / 02687038.2017 .1358352$

Fyndanis, V., Manouilidou, C., Koufou, E., Karampekios, S., \& Tsapakis, E.M. (2013). Agrammatic patterns in Alzheimer's disease: Evidence from tense, agreement, and aspect. Aphasiology, 27, 178-200. Doi: 10.1080/02687038.2012.705814

Fyndanis, V., Varlokosta, S., \& Tsapkini, K. (2012). Agrammatic production: Interpretable features and selective impairment in verb inflection. Lingua, 122, 1134-1147. Doi: 10.1016/j.lingua.2012.05.004

Kok, P., van Doorn, A., \& Kolk, H. (2007). Inflection and computational load in agrammatic speech. Brain and Language, 102, 273-283. Doi: 10.1016/j.bandl.2007.03.001

Miyake, A., Carpenter, P. A., \& Just, M. (1994). A capacity approach to syntactic comprehension disorders: Making normal adults perform like aphasic patients. Cognitive Neuropsychology, 11, 671-717. Doi: 10.1080/02643299408251989 
Rao, S.M. (1995). Neuropsychology of multiple sclerosis. Current Opinion in Neurology, 8 , 216-220.

Renauld, S., Mohamed-Saïd, L., \& Macoir, J. (2016). Language disorders in multiple sclerosis: A systematic review. Multiple Sclerosis and Related Disorders, 10, 103-111. Doi: 10.1016/j.msard.2016.09.005 


\title{
Semantic hyper-interference and hyper-faciliation in aphasia: Evidence for activation-based models
}

\author{
Cristina Romani ${ }^{1}$, Raffaele Nappo ${ }^{1-3}$, Ivana Bureca ${ }^{2}$, Gaspare Galati ${ }^{2,3}$ \\ ${ }^{1}$ School of Life \& Health Sciences Aston University, Birmingham, UK \\ ${ }^{2}$ IRCCS Fondazione Santa Lucia, Rome, Italy \\ ${ }^{3}$ Department of Psychology, University of Rome Sapienza
}

\section{Introduction}

Classical anomia is conceived as a difficulty in accessing intact lexical representations from intact semantic representations. These retrieval difficulties have often been explained by relying on the concept of 'levels of activation'. To be produced, a word needs to reach a given level of activation which depends on its initial level and input from the semantic system. Brain damage would reduce activation levels so that either a different word is produced or nothing at all (e.g., Caramazza, 1997; Rapp \& Goldrick, 2000). However, in some people with aphasia (PwA) retrieval impairments could also be caused by difficulties in selecting the right representation from activated competitors. In turn, selection mechanisms could be either lexical-specific or more general, operating across tasks (see Schnur, 2009; Jefferies \& Lambon Ralph, 2006). Consistent with selection difficulties, there have been reports of PwA where performance becomes significantly worse with repetition of semantically related items (e.g., in word production: McCarthy \& Kartsounis 2000; Warrington \& McCarthy 1983; in matching tasks, Forde \& Humphreys, 1997; 2007; in cyclic blocking naming, Biegler et al.,2008; Schnur et al., 2006; Wilshire \& McCarthy,2002; in continuous naming, Harvey et al., 2019). However, evidence of a direct contrast between activation and selection impairments remains limited.

We assessed whether difficulties of activation and selection can be distinguished in naming tasks. Selection difficulties should produce exacerbated interference effects of semantic relatedness. Activation difficulties, instead, may reduce interference because of the benefits that shared activation will exert on target activation. Interference effects were assessed both in terms of speed and accuracy. Additionally, we assessed whether possible selection difficulties were general or specific by contrasting interference effects in naming tasks with interference effects in tasks where the demands for lexical retrieval are minimized (Probe and Stroop tasks).

\section{Methods}

\section{Participants}

We tested 20 PwA (14 males; mean age 58, SD 13; mean education 13, SD 3) and 15 healthy controls (HC) matched for age and education. They were all Italian native speakers. The PwA had all suffered from a left-hemisphere CVA; time post onset varied from 2 to 24 months $($ mean $=6.0 ; \mathrm{SD}=5.0)$. All made semantic paraphasias or showed signs of anomic difficulties in spontaneous speech. Only one participant made phonological errors in naming tasks. They were all recruited and tested at Santa Lucia Foundation in Rome. Not all participants could carry out all experimental tasks; 20 carried out the Continuous Naming task; 11 the Cyclic Blocking task, 14 the Semantic Probe task, and 15 the Stroop task.

\section{Tasks}

The aphasic participants were administered a comprehensive neuropsychological battery as well as four experimental tasks:

A Continuous Naming task (CN, see Howard et al., 2006) presented a continuous sequence of pictures which contained interspersed sets of items belonging to the same semantic categories 
(five item per set in our case). Typically, performance becomes progressively slower with increasing ordinal position among related pictures.

A Cyclic Blocking task (CBN, see Damian et al. 2001) repeatedly presented, in different orders, sets of (four) pictures for naming. The sets could be semantically related or unrelated. Typically, RTs decrease over presentation cycles because of a priming effect, but this is offset by interference in the related sets.

A Semantic Probe (SP)Task presented a list of words, followed by a probe word. The participant had to decide whether the probe word was in the preceding list. We contrasted a related condition, where the words in the memory list were semantically related to each other and to the probe, with an unrelated condition.

A Stroop task contrasted naming the ink colors of words whose meaning was either congruent or incongruent.

For each task, we calculated speed and accuracy indexes of semantic interference by subtracting performance in baseline conditions from performance in conditions of high interference: for CN, positions 4-5 minus positions 1-2; for CBN, related minus unrelated cycles; for SP, related minus unrelated conditions from negative trials; for the Stroop task, incongruent minus congruent conditions. In addition, in order to neutralize any baseline difference, we calculated relative interference indexes for RTs (RRT) by dividing each interference index by performance at baseline.

\section{Results}

In all tasks, results from $\mathrm{HC}$ and from the overall aphasic group replicated typical interference effects. However, when results were considered individually, different patterns emerged. In $\mathrm{CN}$, a subset of $\mathrm{P}_{\mathrm{wA}}(\mathrm{N}=8$; high-interference group) showed speed interference effects which were significantly stronger than the controls. Other $\mathrm{PwA}$, however, $(\mathrm{N}=12$; high-facilitation group) showed normal or even paradoxical effects, with RTs becoming faster with presentations of related items (see Figure 1). Consistent results were shown in CBN. Of the 11 PwA who have carried out both tasks, five showed interference stronger than controls in both tasks and four showed normal or weaker interference than controls in both tasks. There was no group difference when interference effects were considered in terms of errors. There was also no group difference in severity at baseline (RT or errors). However, the group with evidence of reduced interference/more facilitation, made significantly more lexical errors across naming tasks and more perseverative errors. Participants who showed stronger interference did so despite no semantic impairment in the Pyramids and Palm Trees Test and no increased interference in the SP or Stroop task.

\section{Discussion}

Our results can be explained by assuming that some PwA suffer from selection difficulties which increase with the number of competitors. These impairments affect specifically selection mechanisms operating within the lexicon. Other PwA, instead, suffer from activation difficulties and for this reason benefit more than usual from the activation the target receives from semantic neighbors. This shared activation will neutralize or even offset any increased difficulties with selection, but at the cost of making more semantic errors (see Chen \& Mirman, 2013 for a simulation where low activation is linked to facilitation and high activation to interference). Our results support lexical-retrieval models where semantic-neighbours can be friends or foes depending on task demands and the state of the system. They do not support incremental 

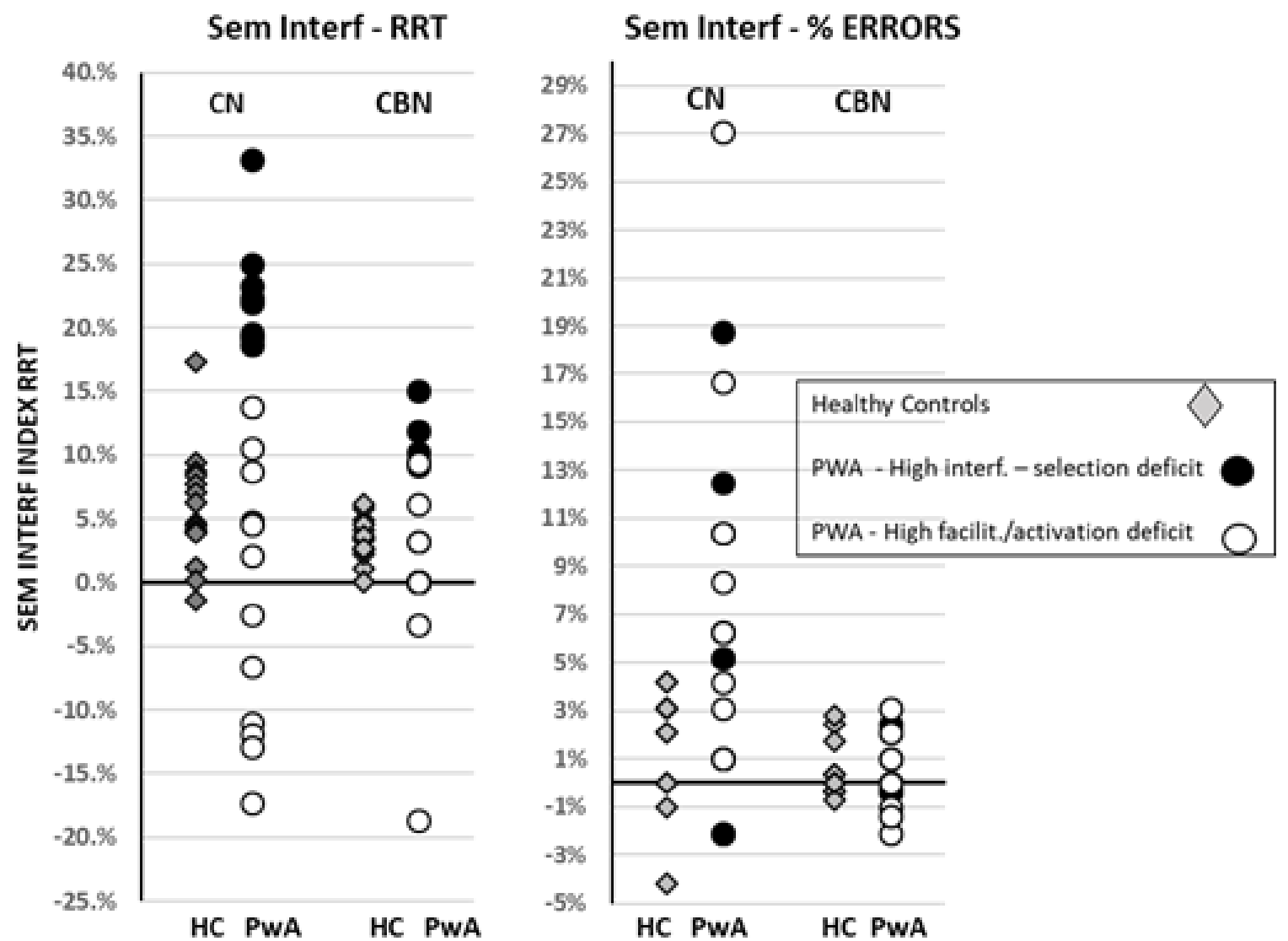

Figure 1: Semantic interference effect in Continuous Naming (CN) and Cyclic blocking Naming $(\mathrm{CBN})$

\begin{tabular}{|c|c|c|c|c|c|c|c|c|c|c|c|}
\hline \multirow[b]{2}{*}{ Participant } & \multirow[b]{2}{*}{ Group } & \multicolumn{3}{|c|}{ CONTINUOUS NAMING SPEED } & \multicolumn{2}{|c|}{ CYCLIC BLOCKING } & \multicolumn{5}{|c|}{ CONTINUOUS NAMING \% ERRORS } \\
\hline & & $\begin{array}{l}\text { RT Baseline } \\
\text { (Position 1) }\end{array}$ & $\begin{array}{l}\text { Interf Index - } \\
\text { RRTs }\end{array}$ & $\begin{array}{l}\text { Diff from } \\
\text { controls }\end{array}$ & $\begin{array}{l}\text { Interf Index - } \\
\text { RRTs }\end{array}$ & $\begin{array}{l}\text { Diff from } \\
\text { controls }\end{array}$ & $\begin{array}{l}\text { Total } \\
\text { errors }\end{array}$ & $\begin{array}{l}\text { All lexical } \\
\text { errors }\end{array}$ & $\begin{array}{l}\text { Errors Baseline } \\
\text { (Position 1) }\end{array}$ & Interf Index & $\begin{array}{l}\text { Diff from } \\
\text { controls }\end{array}$ \\
\hline AS & HI-SD & 1709 & $23 \%$ & ** & $10 \%$ & ** & $10 \%$ & $5 \%$ & $8 \%$ & $5 \%$ & ns \\
\hline BP & HI-SD & 4200 & $33 \%$ & ** & $9 \%$ & * & $20 \%$ & $7 \%$ & $23 \%$ & $-2 \%$ & ns \\
\hline$C R$ & HI-SD & 1469 & $20 \%$ & ** & $14 \%$ & ** & $10 \%$ & $5 \%$ & $15 \%$ & $1 \%$ & ns \\
\hline CeM & HI-SD & 3388 & $23 \%$ & ** & -- & -- & $22 \%$ & $3 \%$ & $21 \%$ & $19 \%$ & ** \\
\hline $\mathrm{OL}$ & HI-SD & 1494 & $19 \%$ & ** & $11 \%$ & ** & $3 \%$ & $2 \%$ & $6 \%$ & $0 \%$ & ns \\
\hline $\mathrm{RR}$ & HI-SD & 4435 & $22 \%$ & $* *$ & - & -- & $24 \%$ & $11 \%$ & $25 \%$ & $13 \%$ & $* *$ \\
\hline SBK & HI-SD & 2943 & $19 \%$ & ** & $\mathbf{9 \%}$ & * & $8 \%$ & $1 \%$ & $25 \%$ & $5 \%$ & ns \\
\hline SM & HI-SD & 2396 & $25 \%$ & ** & & & $7 \%$ & $4 \%$ & $13 \%$ & $-2 \%$ & ns \\
\hline Mean & HI-SD & 2754 & $23 \%$ & & $11 \%$ & & $13 \%$ & $5 \%$ & $17 \%$ & $5 \%$ & \\
\hline $\mathrm{BC}$ & HP-AD & 2405 & $5 \%$ & ns & $10 \%$ & $*$ & $44 \%$ & $16 \%$ & $54 \%$ & $10 \%$ & ** \\
\hline CG & HP-AD & 2938 & $-7 \%$ & $* *$ & - & -- & $25 \%$ & $8 \%$ & $21 \%$ & $10 \%$ & ** \\
\hline CM & HP-AD & 2605 & $5 \%$ & ns & $-4 \%$ & *** & $9 \%$ & $5 \%$ & $21 \%$ & $3 \%$ & ns \\
\hline Cas & HP-AD & 1781 & $-12 \%$ & ** & $3 \%$ & ns & $8 \%$ & $7 \%$ & $8 \%$ & $6 \%$ & ns \\
\hline $\cos$ & HP-AD & 1809 & $14 \%$ & ns & -- & -- & $30 \%$ & $17 \%$ & $31 \%$ & $8 \%$ & ${ }^{*}$ \\
\hline $\mathrm{DL}$ & HP-AD & 2907 & $-13 \%$ & ** & -- & -- & $19 \%$ & $3 \%$ & $8 \%$ & $27 \%$ & ** \\
\hline GG & HP-AD & 2737 & $-3 \%$ & * & -- & -- & $11 \%$ & $7 \%$ & $23 \%$ & $0 \%$ & ns \\
\hline GM & HP-AD & 1079 & $9 \%$ & ns & $3 \%$ & & $12 \%$ & $10 \%$ & $13 \%$ & $6 \%$ & ns \\
\hline PS & HP-AD & 876 & $5 \%$ & ns & - & -- & $37 \%$ & $21 \%$ & $46 \%$ & $17 \%$ & ns \\
\hline $\mathrm{RP}$ & HP-AD & 2116 & $2 \%$ & ns & $6 \%$ & ns & $6 \%$ & $7 \%$ & $6 \%$ & $6 \%$ & ns \\
\hline sc & HP-AD & 3075 & $-17 \%$ & $* *$ & $-20 \%$ & $* *$ & $10 \%$ & $8 \%$ & $4 \%$ & $10 \%$ & $* *$ \\
\hline SP & HP-AD & 3874 & $-11 \%$ & ** & & & $22 \%$ & $13 \%$ & $25 \%$ & $4 \%$ & ns \\
\hline Mean & HP-AD & 2350 & $-2 \%$ & & $0 \%$ & & $19 \%$ & $10 \%$ & $22 \%$ & $\mathbf{9 \%}$ & \\
\hline \multirow{2}{*}{$\begin{array}{l}\text { Healthy } \\
\text { Controls }\end{array}$} & Mean & & $6 \%$ & & $4 \%$ & & & & & $2 \%$ & \\
\hline & SD & & $4 \%$ & & $2 \%$ & & & & & $2 \%$ & \\
\hline \multicolumn{2}{|c|}{ Aphasic sub-groups diff } & 0.39 & $<.001$ & & 0.05 & & 0.23 & 0.02 & 0.45 & 0.21 & \\
\hline
\end{tabular}


learning models where, after target selection, semantic-lexical connections of activated neighbours are semi-permanently weakened (see Oppenheim et al., 2012; Navarrate et al., 2012). This is incompatible with enduring facilitation effects.

\section{References}

Biegler, K.A., Crowther J.E., \& Martin R.C. (2008). Consequences of an inhibition deficit for word production and comprehension: Evidence from the semantic blocking paradigm. Cognitive Neuropsychology, 25:4, 493-527, DOI: 10.1080/02643290701862316

Caramazza, A. (1997). How may layers are there in lexical access? Cognitive Neuropsychology, 14, 177-208.

Chen Q., \& Mirman D. (2012). "Competition and cooperation among similar representations: Toward a unified account of facilitative and inhibitory effects of lexical neighbors". Psychological Review, 119, 898-898. doi: 10.1037/a0030049

Damian M.F., Vigliocco G, \& Levelt W.J.M. (2001). Effects of semantic context in the naming of pictures and words. Cognition, 81, B77-B86. doi: 10.1016/S0010-0277(01)00135-4

Forde, E., \& Humphreys GW (1997). A Semantic Locus for Refractory Behaviour: Implications for Access Storage Distinctions and the Nature of Semantic Memory. Cognitive Neuropsychology, 14, 367-402. doi: 10.1080/026432997381529

Goldrick, M. \& Rapp, B. (2002). A residual interaction account (RIA) of spoken word production. The best of both worlds. Aphasiology, 16, 20-55.

Harvey, D.Y., Traut, H.J. \& Middleton E.L. (2019). Semantic interference in speech error production in a randomised continuous namingtask: evidence from aphasia. Language, Cognition and Neuroscience, 34 (1), 69-86, DOI:10.1080/23273798.2018.1501500

Howard, D., Nickels, L., Coltheart, M., \& Cole-Virtue, J. (2006). Cumulative semantic inhibition in picture naming: experimental and computational studies. Cognition, 100, 464482. doi: 10.1016/j.cognition.2005.02.006

McCarthy, A., \& Kartsounis, D. (2000). Wobbly words: Refractory anomia with preserved semantics. Neurocase, 6, 487-497. doi: 10.1093/neucas/6.6.487

Navarrete, E., Prato, D., \& Mahon, B.Z. (2012). Factors determining semantic facilitation and interference in the cyclic naming paradigm. Front Psychol, 3,1-15. doi: 10.3389/fpsyg.2012.00038

Oppenheim, G.M., Dell, G.S., \& Schwartz, M.F. (2010). The dark side of incremental learning: A model of cumulative semantic interference during lexical access in speech production. Cognition, 114, 227-252. doi: 10.1016/j.cognition.2009.09.007

Schnur T.T., Schwartz M.F., Brecher A., \& Hodgson, C. (2006). Semantic interference during blocked-cyclic naming: Evidence from aphasia. J Mem Lang 54:199-227. doi: 10.1016/j.jml.2005.10.002

Schnur, TT, Schwartz, M.F., \& Kimberg D.Y., et al (2009). Localizing interference during naming: convergent neuroimaging and neuropsychological evidence for the function of Broca's area. Proceedings National Academy of Science USA, 106, 322-327. doi: $10.1073 /$ pnas.0805874106

Warrington, E.K., \& McCarthy R.A. (1983). Category specific access dysphasia. Brain, 106, 859 -878. (doi:10.1093/brain/106.4.859)

Wilshire, C.E.W., \& McCarthy R. (2002). Evidence for a context-sensitive word retrieval disorder in a case of nonfluent aphasia. Cognitive Neuropsychology, 19, 165-86. doi: $10.1080 / 02643290143000169$ 


\title{
The influence of verbal short-term memory capacity on microlinguistic measures of word and utterance level content in discourse
}

\author{
Jessica Obermeyer, Nadine Martin \\ Eleanor M. Saffran Center for Cognitive Neuroscience Department of Communication \\ Sciences and Disorders, Temple University
}

\begin{abstract}
Introduction
Modern aphasia theory posits that cognitive abilities are required for language tasks (Hula \& McNeil, 2008; Martin \& Saffran 1992; 1997). One of these cognitive factors is verbal short term memory (VSTM). Evidence suggests that VSTM is an integral component of word processing and production that allows for lexical items to be maintained/stored temporarily to carry out linguistic processes. People with aphasia demonstrate reduced VSTM (Martin \& Saffran, 1997) which has been found to influence their single word comprehension and production (Minkina, et al., 2017). However, there are few accounts that evaluate the interaction between VSTM and discourse production in people with aphasia (PWA). Cahana-Amitay and Jenkins (2018) explored the relationship between working memory and discourse in PWA and reported that word and sentence span was predictive of macrolinguistic measures (global coherence (i.e. topic maintenance), local coherence (i.e. relationship between utterances), narrative organization), but not microlinguistic ability (lexical diversity and narrative length). The current project evaluated the contribution of VSTM to microlinguistic discourse measures that evaluate word and utterance level content and basic sentence structure. The aims of this study were:

1) to evaluate the relationship between VSTM and word level relevant content in discourse (e.g. words that are accurate and relevant to the stimuli (Correct information units; Nicholas \& Brookshire, 1993)).

2) to evaluate the relationship between VSTM and utterance level a) relevance, and b) basic structure as measured by Complete Utterances (CUs; Edmonds, et al., 2009).
\end{abstract}

We hypothesized that VSTM would be related to word and utterance level content in discourse production due to previous findings that VSTM is related to word retrieval abilities in isolation (Wilshire, Keall, \& O'Donnell, 2010; Minkina, 2015). We did not predict a relationship between the production of basic sentence structure and VSTM because we expected the majority of short term storage would be related to maintaining activation of lexical items, which occurs earlier in the process of discourse planning/production and would therefore require more temporary storage (Sherrat, 2007).

\section{Methods}

Participants

Participants included 23 native English speakers at least 6-months post left hemisphere damage. Aphasia was diagnosed by the Western Aphasia Battery-Revised (Kertez, 2001) (average Aphasia Quotient=78.38, standard deviation.=8.86).

\section{Procedures}

Data were collected as part of an ongoing study. Participants completed discourse sampling during one-two sessions which included ten prompts, and the elicitation procedure described by Nicholas and Brookshire (1993). Discourse was audio recorded and orthographically transcribed. Reliability was completed on $20 \%$ of transcripts with $95.9 \%$ accuracy. Transcripts were then coded for words, Correct Information Units (CIUs; Nicholas and Brookshire, 1993) and Complete Utterances (CUs; Edmonds, et al., 2009). CIUs were defined as words that are 
relevant to the stimuli and not repeated. CUs are an utterance level measure in which utterances are relevant to the stimuli (REL) and contain basic $\mathrm{S}+\mathrm{V}+(\mathrm{O})$ structure (SV). Point-bypoint coding reliability was completed for $20 \%$ of transcripts and revealed $98.12 \%$ accuracy for words, $91.81 \%$ for CIUs, and $87.28 \%$ for CUs.

VSTM was evaluated using three tasks, a word pointing span, category coordinate span and a rhyming span. In the word pointing span task participants heard lists of words and pointed to the corresponding pictures, in a field of 9 , in the exact order they heard them. Lists started at a single word and increased to 7 words. Once a participant scored $<50 \%$ accuracy on a word list, they did not continue to the next list. The category span required participants to listen to a word list, then hear an additional word and respond yes/no if any of the words in the list were in the same category as the final word they heard. The rhyming span was completed similarly with participants responding yes/no if the final word they heard rhymed with any of the words in the list. For the category and rhyming span task, participants did not move on to the next highest span when they scored $<75 \%$ accuracy on yes or no response lists (higher accuracy requirement due to yes/no response). A nonverbal short term memory task, the forward Corsi block span, was also administered. To complete this task, the examiner pointed to individual blocks arranged spatially and then the participant was instructed to point to the same series of blocks. The task began with a single block and increased to up to nine blocks. Once the participant scored $<50 \%$, they do not continue to the next highest list. Only 20 participants completed this task due to examiner error.

\section{Results}

The following variables were evaluated: \%CIUs, \%CUs, \%REL, \%SV, word pointing span, category coordinate span, and rhyming span. Partial Pearson Correlation analysis was completed to evaluate the relationships between word and utterance level discourse measures and VSTM tasks, while controlling for nonverbal cognitive ability and general severity with a nonverbal span task (forward Corsi Block). Analysis revealed significant correlations between word pointing span and \%CIUs ( $\mathrm{r}=.673, p=.003)$, \%REL $(\mathrm{r}=.612, p=.009)$ and \%CUs $(\mathrm{r}=.522, p=.032)$. See table 1. The Category coordinate and Rhyming span were not correlated with any of the discourse measures, but were correlated with each other $(\mathrm{r}=.517, p=.034)$.

Table 1. Partial Correlation Results for Verbal Short Term Memory and Discourse Measures Controlling for Nonverbal Short-term Memory Span

\begin{tabular}{llllllll}
\hline Variable & 1 & 2 & 3 & 4 & 5 & 6 & 7 \\
\hline 1. Word pointing span & - & - & - & - & - & - & - \\
2. Category Coordinate & .362 & - & - & - & - & - & - \\
3. Rhyming & .004 & $.517^{*}$ & - & - & - & - & - \\
4. \% CIUs & $.673^{*}$ & .411 & .168 & - & - & - & - \\
$5 . \%$ CUs & $.522^{*}$ & .313 & -.175 & $.555^{*}$ & - & - & - \\
$6 . \%$ REL & $.612^{*}$ & .340 & .119 & $.923^{*}$ & $.600^{*}$ & - & - \\
$7 . \%$ SV & .179 & .199 & -.271 & .144 & $.834^{*}$ & .132 & - \\
\hline
\end{tabular}

Note. ${ }^{*}=$ significant correlation at $p<.05 ; \%$ CIUs=percent of correct information units; \% CUs=percent of complete utterances; \% REL=percent of relevant utterances; \% SV=percent of utterances with subject+ verb+(object) structure.

\section{Discussion}

The current study sought to evaluate potential relationships between VSTM ability and microlinguistic discourse outcomes at the word and utterance level. We anticipated that word and utterance level relevant content would be related to VSTM ability. Our findings partially support this hypothesis. Word pointing span was moderately significantly correlated with word 
level relevance (\%CIUs), utterance level relevance (\%REL), and the complete utterance measure (\%CUs) which evaluates relevance and structure, while controlling for nonverbal short-term memory ability (forward corsi block span). The category and rhyming span were not related to discourse relevance at the word or utterance level. One potential explanation is that the probe span tasks require temporary storage and recognition opposed to storage and recall which is required by the word pointing span. As such, the word pointing span is more likely to share skills with production tasks such as discourse.

\section{References}

Cahana-Amitay, D., \& Jenkins, T. (2018). Working memory and discourse production in people with aphasia. Journal of Neurolinguistics, 48, 90-103.

Edmonds, L. A., Nadeau, S. E., \& Kiran, S. (2009). Effect of Verb Network Strengthening Treatment (VNeST) on lexical retrieval of content words in sentences in persons with aphasia. Aphasiology, 23(3), 402-424.

Hula, W. D., \& McNeil, M. R. (2008, August). Models of attention and dual-task performance as explanatory constructs in aphasia. In Seminars in speech and language (Vol. 29, No. 03, pp. 169-187). Thieme Medical Publishers.

Kertesz, A. (2007). Western Aphasia Battery-Revised. PsychCorp. San Antonio, TX.

Martin, N., \& Saffran, E. M. (1997). Language and Auditory-verbal Short-term Memory Impairments: Evidence for Common Underlying Processes, Cognitive Neuropsychology, $14(5), 641-682$.

Martin, N., \& Saffran, E. M. (1992). A computational account of deep dysphasia: Evidence from a single case study. Brain and language, 43(2), 240-274.

Minkina, I., Rosenberg, S., Kalinyak-Fliszar, M., \& Martin, N. (2017, February). Short-term memory and aphasia: From theory to treatment. In Seminars in speech and language (Vol. 38, No. 01, pp. 017-028). Thieme Medical Publishers.

Nicholas, L. E., \& Brookshire, R. H. (1993). A system for quantifying the informativeness and efficiency of the connected speech of adults with aphasia. Journal of Speech, Language, and Hearing Research, 36(2), 338-350.

Sherratt, S. (2007). Multi-level discourse analysis: A feasible approach. Aphasiology, 21(3-4), 375-393.

Wilshire, C. E., Keall, L. M., \& O’Donnell, D. J. (2010). Semantic contributions to immediate serial recall: Evidence from two contrasting aphasic individuals. Neurocase, 16(4), 331351. 


\section{Application of the Nonverbal Semantics Test (NVST) to persons with aphasia after stroke and persons with dementia}

Katharina Hogrefe ${ }^{1}$, Wolfram Ziegler ${ }^{1}$, Ralf Glindemann ${ }^{1}$, Madleen Klonowski ${ }^{2}$, Edith Wagner-Sonntag ${ }^{2}$, Gudrun Klingenberg ${ }^{3}$, Janine Diehl-Schmid ${ }^{4}$, Carola Roßmeier ${ }^{4}$, Adrian Danek $^{5}$, Johannes Levin ${ }^{5}$, Catharina Prix ${ }^{5}$, Sandra Loosli ${ }^{5}$, Elisabeth Wlasich ${ }^{5}$, \& Georg Goldenberg $^{3}$

${ }^{1}$ Clinical Neuropsychology Research Group, Institute of Phonetics and Speech Processing, Ludwig-Maximilians-Universität München

${ }^{2}$ Schön Klinik München Schwabing

${ }^{3}$ Schön Klinik Bad Aibling Harthausen

${ }^{4}$ Klinik für Neurologie, Klinikum Rechts der Isar, Technical University Munich

${ }^{5}$ Neurologische Klinik und Poliklinik, Ludwig-Maximilians-Universität München

\section{Introduction}

Semantic processing is essential for human communication, e.g. for initiating verbal utterances and nonverbal expressions like gestures or drawings. Assessment of semantic processing capacities typically relies on verbal tasks (e.g. word-picture-matching), which are sensitive, however, to impairments on several language processing stages. Hence, there is a strong need for a tool that measures semantic processing skills independent of verbal abilities. Furthermore, semantic processing should be tested in different nonverbal modalities, considering that modality specific cortical regions interact with an amodal central representational $H u b$ whenever semantic processing occurs (Hub and Spokes Model; e.g. Rogers et al., 2004; Lambon Ralph et al., 2017).

To meet these requirements, we extended three clinically established tasks (Glindemann et al., 2002; Goldenberg, 2003) to an assessment tool for nonverbal semantic processing skills. The Nonverbal Semantics Test (NVST; Hogrefe et al., in prep.) is based on three different paradigms:

- a semantic sorting task requiring participants to recognize semantic relationships,

- a drawing task requiring participants to depict the salient features of an object,

- a pantomime task requiring participants to demonstrate the use of an object.

Semantic Sorting is mainly a receptive task, whereas Drawing and Pantomime require the depiction of semantic information. NVST scores can be transformed into differential norms respecting age, gender, and education. Criterion-based NVST norms were derived from a calibration sample of 192 neurologically healthy participants stratified for sex, age, and education level.

A study including persons with aphasia after stroke showed that performance in these tasks predicts the ability to communicate in a multimodal role play task (Jakob, 2012).

In this study, we compare the performance of healthy persons and two groups of persons with brain damage on the three subtests of the Nonverbal Semantics Test. Furthermore, we determined neuropsychological and neurolinguistic factors influencing the nonverbal capacities probed by the NVST.

\section{Methods}

\section{Participants}

The study included 192 persons without brain damage, stratified for sex, age, and education (the NVST calibration sample). Furthermore, we present preliminary data from 82 persons 
with brain damage:

- 34 persons with severe aphasia after first ever cerebrovascular accident (CVA)

- 48 persons with neurodegenerative disorders, among them

- 17 persons with Alzheimer's dementia and

- 30 persons with frontotemporal dementia (FTD), namely with

* behavioural variant FTD, $\mathrm{N}=8$

* semantic variant PPA, $\mathrm{N}=8$

* nonfluent / agrammatic variant PPA, $\mathrm{N}=7$

* other FTD-types, $\mathrm{N}=7$

Assessment

We administered to

- all participants: the Nonverbal Semantics Test (NVST),

- all participants with brain damage: two language tasks from the LEMO-battery (De Bleser et al., 2004): word-to-picture matching and confrontation naming.

- participants with severe aphasia after stroke: the Aachen Aphasia Test (AAT)

- participants with dementia: the CERAD (Consortium to establish a registry for Alzheimer's disease) test battery.

\section{Statistical Analyses}

A linear mixed effects model analysis was conducted on the patients' standardized NVST-scores, with GROUP (CVA vs. neurodegenerative disorders) and SUBTEST (Semantic Sorting, Pantomime, Drawing) as fixed factors and PARTICIPANTS as random intercept factor. This analysis aimed to compare the three NVST subtests for their sensitivity as a function of the underlying disorder. Significance levels were assessed by testing the full linear model against models in which the respective factors were eliminated (Table 1).

Moreover, the dementia group was examined for differences between the four largest subgroups, i.e., Alzheimer's disease, semantic variant PPA, behavioural variant FTD, and nonfluent / agrammatic variant PPA.

Finally, mixed effects linear regression analyses were conducted for each patient group to determine the factors influencing semantic processing as assessed by the Nonverbal Semantics Test.

\section{Results}

Figure 1 depicts standardized scores of the three NVST subtests as a function of etiology (CVA vs. neurodegenerative disorders). As a result of the applied standardization transform, $95 \%$ of the healthy participants scored below 1. The linear mixed effects model revealed higher standard scores, i.e., greater impairment, in the stroke patients relative to the patients with neurodegenerative disorders $\left(\mathrm{X}^{2}(3)=11.9, p<.01\right)$. Of note, the three NVST-subtests proved equally sensitive across both groups $\left(\mathrm{X}^{2}(4)=8.8, p=.07\right)$, yet with a significant interaction of GROUP by SUBTEST $\left(\mathrm{X}^{2}(2)=6.3, p<.05\right)$. The interaction resulted from a greater vulnerability of the Pantomime task in the patients with aphasia after stroke as compared to the dementia patients.

Separate Kruskal-Wallis-analyses involving only the group with dementia revealed no significant differences between the four subgroups in either subtest (Semantic Sorting: $\mathrm{X}^{2}(3)=4.4$, p>.05; Pantomime: $\mathrm{X}^{2}(3)=3.0, \mathrm{p}>.05$; Drawing: $\left.\mathrm{X}^{2}(3)=4.5, \mathrm{p}>.05\right)$. Closer inspection of the data indicated a trend for lower performance of the subgroup with semantic variant PPA. 


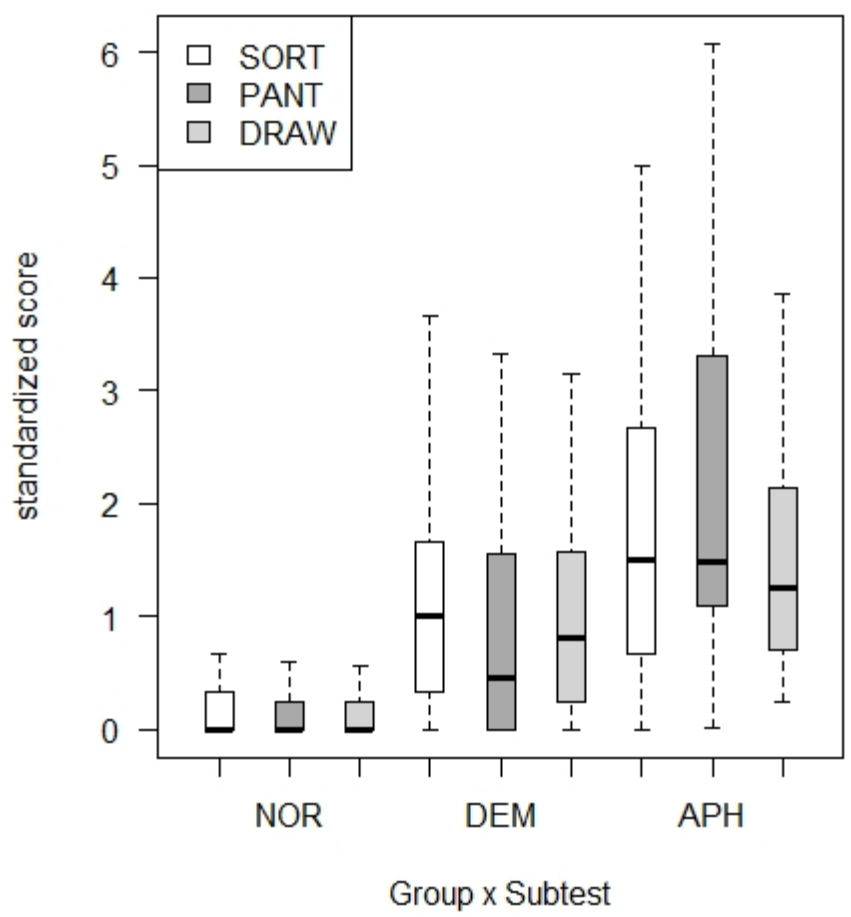

Figure 1. Standardized scores of the three NVST subtests by participant groups. By virtue of the standardization transform, healthy participants score lower than 1.

Regression analyses showed that none of the AAT-subtests was as a predictor of Semantic Sorting and Pantomime in the group with severe aphasia (CVA). Drawing scores were predicted by the AAT-subtests Token Test and Written Language, though with a low $\mathrm{R}^{2}(.41)$.

For persons with dementia, the naming task of the CERAD battery was the only predictor of the performance in the Semantic Sorting Task $\left(\mathrm{R}^{2}=.49\right)$. The visuo-constructive task of the CERAD battery was the only predictor of the performance in the NVST Drawing Task $\left(\mathrm{R}^{2}=.63\right)$.

\section{Discussion}

The three NVST subtests proved to have comparable sensitivity across 82 participants with aphasia after stroke and dementia. The Pantomime task was more vulnerable to aphasia after stroke than to neurodegenerative disorder.

The lack of differences between the dementia subgroups can possibly be explained by the small sample size.

In the aphasic patients examined here, performance on the Token Test and the Written Language subtest of the AAT were weakly associated with the NVST Drawing score, while no AAT parameters were found to predict the Pantomime and Semantic Sorting scores.

The Nonverbal Semantics Test (NVST) is a standardized tool for the clinical assessment of nonverbal semantic abilities. It is easy to administer, time-efficient, and can be applied to participants with even severe language disorders. It measures abilities that are not captured by the standard aphasia assessment parameters. The Nonverbal Semantics Test should be ad- 
ministered along with aphasia assessment to enable a tailored treatment planning.

\section{References}

De Bleser, R., Cholewa, J., Stadie, N., Tabatabaie, S. (2004). LEMO-Lexikon modellorientiert. Einzelfalldiagnostik bei Aphasie, Dyslexie und Dysgraphie. München: Urban \& Fischer / Elsevier.

Glindemann, R., Klintwort, D., Ziegler, W., \& Goldenberg, G. (2002). Bogenhausener SemantikUntersuchung. München: Urban \& Fischer.

Goldenberg, G. (2003). Pantomime of object use: a challenge to cerebral localization of cognitive function. NeuroImage, 20, S101-S106.

Jakob, H. (2012). Evaluation eines Diagnostikverfahrens zur Untersuchung verbaler und nonverbaler Kommunikationsfähigkeiten bei Aphasie -Der Szenariotest. Unpublished Master's thesis. Ludwig-Maximilians-Universität München.

Lambon Ralph, M.A., Jefferies, E., Patterson, K., \& Rogers, T.T. (2017). The neural and computational bases of semantic cognition. Nature, 18, 42-55.

Hogrefe, K., Glindemann, R., Ziegler, W., \& Goldenberg, G. (in prep.). Nonverbaler Semantiktest (NVST). Göttingen: Hogrefe.

Huber, W., Poeck, K., Weniger, D., \& Willmes, K. (1983). Aachener Aphasie Test. Göttingen: Hogrefe.

Rogers, T.T., Lambon Ralph, M.A., Garrard, P., Bozeat, S., McClelland, J.L., Hodges, J.R., \& Patterson, K. (2004). The structure and deterioration of semantic memory: A neuropsychological and computational investigation. Psychol. Rev., 111, 205-235. 


\title{
Treatment of adjectives in aphasia: Two case-studies
}

\author{
Kati Renvall ${ }^{1,2}$, Lyndsey Nickels ${ }^{2}$ \\ ${ }^{1}$ Department of Psychology and Speech-Language Pathology, University of Turku, Finland \\ ${ }^{2}$ Department of Cognitive Science, Macquarie University, Australia
}

\section{Introduction}

Most treatment studies on aphasia target naming of relatively concrete nouns and verbs. In the majority of the studies, item-specific treatment effects are reported but signs of generalisation are scarce (e.g., Wisenburn \& Mahoney, 2009). While there are studies suggesting that targeting more abstract vocabulary might enhance generalisation from treated to untreated words (e.g., Kiran, Sandberg, \& Abbott, 2009), there are very few studies targeting truly abstract vocabulary, such as emotive adjectives (e.g., sad, regretful, ecstatic). In a single-case treatment study (Renvall \& Nickels, 2019), we provided the first evidence that production of emotive adjectives may be enhanced by treatment. Here we attempt to replicate these results in two further people with aphasia (PWA).

\section{Methods}

Participants

Two people with chronic aphasia and word-finding difficulty participated in the study. DEH, a 68-year-old male, presented with a phonologically-based impairment (Boston Naming Test 33/60). ALM, a 77-year-old male presented with a mixed semantic-phonological impairment (Boston Naming Test 22/60).

\section{Treatment materials, design and statistical analyses}

We report results on two sets of stimuli used to elicit emotive adjectives. Both tasks required participants to produce as many single-words as possible that were related to feelings/emotions exhibited by the person/people depicted.

For the 'Emotions-in-Context' task, we selected 35 of 48 Emotions ColorCards@ (1996) approximately one half depicting positive and the other half negative emotions. The same 35 pictures were also used in treatment, each picture with three spoken words, giving a total of 105 treated labels. The labels were selected based on their appropriateness from our normative data and on the participants' baseline performance. The Emotions-in-Context task was administered three times before, three times during and three times after the treatment (Post1 immediately after, Post2 one-week after, and Post3 three weeks after for DEH and four weeks after for ALM).

The second set of items ('Basic Emotions') were not used in treatment but used to track possible treatment effects. The set used 12 pictures from the Karolinska Directed Basic Emotions (Lundqvist, Flykt, \& Öhman, 1998). Different from the Emotion-in-Context task, only single face without any contextual cues were visible on each picture and all pictures depicted as clearly as possible one of the basic emotions (i.e., happiness, sadness, anger, fear, surprise, disgust). This task was administered three times before and two times after the treatment (at Post1 and Post3 time points). For both tasks, all labels produced within 30 seconds (Emotions-inContext) or 60 seconds (Basic Emotions) were transcribed and scored.

Treatment was administered as computer-implemented home-practice together with weekly meetings with the therapist. The participants were provided with laptop computers and PowerPoint files including 35 target pictures from the set of Emotions ColorCards@ (1996). Each slide in the PowerPoint presented one target picture with audio-recordings of three selected 
labels suitable for describing the feeling/emotion on the face of the person/people in the picture. The participants were asked to listen to each recording and repeat the label immediately afterwards.

Data were analysed using Weighted Statistics (WEST; Howard, Best, \& Nickels, 2015). We considered the treatment to be effective only when there was both a significant positive trend (WEST-Trend) and significantly greater improvement during treatment than no treatment phases (WEST-Rate of Change (ROC)).

\section{Results}

\section{Treated pictures (Emotions-in-Context)}

DEH showed significant treatment effects with improved production of treated and untreated labels for the Emotions-in-Context pictures: treated labels, WEST-Trend: $t(34)=4.24, p<$ .001 ; WEST-ROC: $t(34)=3.01, p=.002$; untreated labels, WEST-Trend: $t(34)=5.71, p<$ $.001 ;$ WEST-ROC: $t(34)=1.83, p<.04$.

ALM did not show unambiguous evidence for improvement for treated labels for the Emotionsin-Context pictures, with greater improvement in the treatment phase than the untreated phase, but the overall trend for improvement just failing to reach significance: WEST-Trend: $t(34)=1.50, p=.07$; WEST-ROC: $t(34)=3.82, p<.001$. However, he showed significant treatment-related improvement for untreated labels: WEST-Trend: $t(34)=5.10, p<.001$; WEST-ROC: $t(34)=5.52, p<.001$.
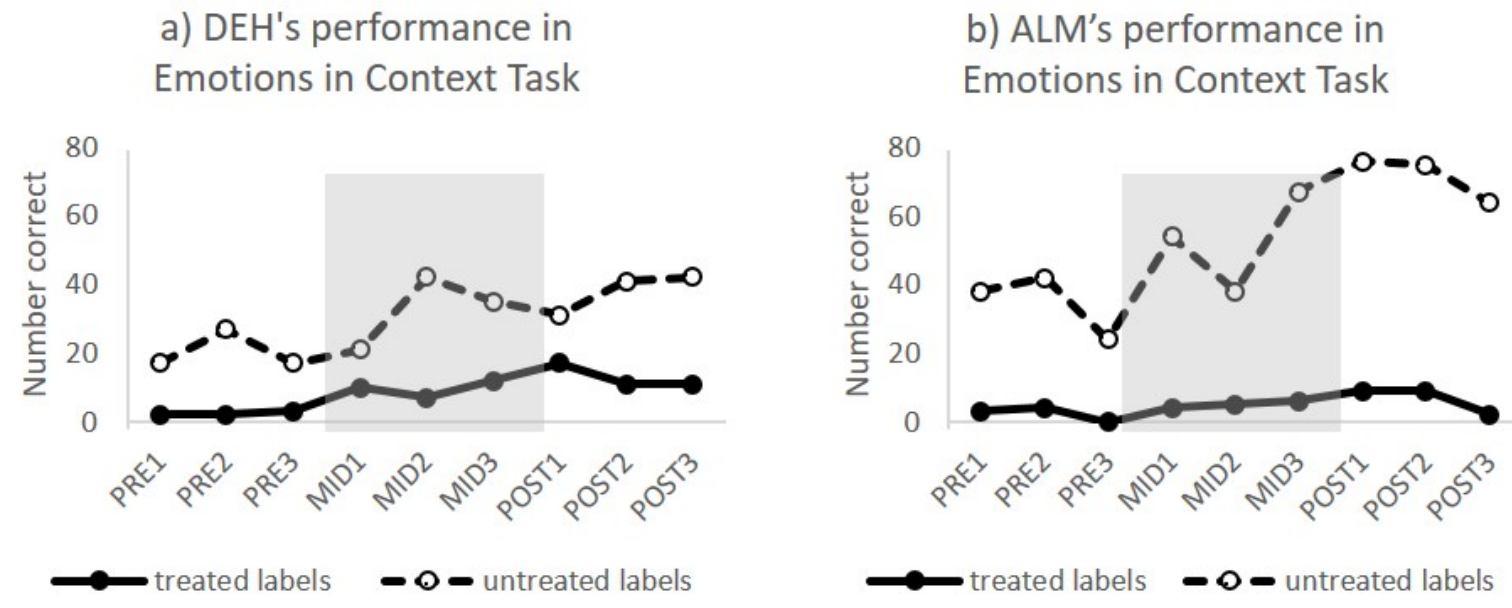

Figure 1. DEH's (a) and ALM's (b) performance in producing treated and untreated labels in the Emotions in Context Task (treatment phase shaded).

\section{Untreated pictures (Basic Emotions)}

In the Basic Emotions task, both participants showed significant treatment-related improvement in the production of treated labels to these untreated pictures (DEH: WEST-Trend: $t(11)=2.79, p=.009$; WEST-ROC: $t(11)=2.11, p=.03 ;$ ALM: WEST-Trend: $t(11)=4.52, p<$ .001 ; ALM WEST-ROC: $t(11)=2.92, p=.007)$. However, there was no evidence for treatmentrelated improvement for untreated labels for either participant (DEH WEST-Trend: $t(11)=-$ $1.80, p=.95 ;$ WEST-ROC: $t(11)=0.64, p=.30 ;$ ALM WEST-Trend: $t(11)=1.21, p=.13$; WEST-ROC: $t(11)=1.42, p=.09)$.

\section{Discussion}

We replicated the treatment for emotive adjectives that we had previously undertaken with 
'GEC' (Renvall \& Nickels, 2019) with two new participants, both of whom, like GEC, showed treatment-related improvements in their adjective production.

While DEH showed treatment-related improvement in the production of treated adjectives for treated pictures, ALM's improvement was not (quite) significant. This discrepancy might be due to ALM's greater semantic problems and more severe word retrieval impairment. Despite this, both DEH and ALM, unlike our first case GEC, produced more treated labels with untreated pictures. This shows that they had not simply learned a pairing between a lexical form and a particular picture. Instead, it is likely that they have learned (or strengthened) the mapping between the concept and the lexical form, enabling them to use the word form in other appropriate contexts.

Interestingly, and again in contrast to GEC, both participants also produced more untreated labels for treated pictures. However, there was no significant treatment effect for untreated labels in the untreated pictures. Consistent with the item-specific effects of lexical retrieval treatment in general, this suggests that the treatment did not result in generally improved accessibility for emotive adjectives. Instead, perhaps the treatment increased the participants' conceptualisation of the emotions in the treated pictures, in turn, this may have increased the activation from semantics to the lexical forms for appropriate (untreated) labels for these pictures.

In sum, this study has demonstrated that retrieval of emotive adjectives can be successfully treated in PWA and that some generalisation of treatment effects is possible.

\section{References}

Emotions ColorCards@ (1996). First new edition 1996 (reprinted 2008, 2010, 2011, 2912). Milton Keynes, UK: Speechmark Publishing.

Howard, D., Best, W., \& Nickels, L. (2015). Optimising the design of intervention studies: Critiques and ways forward. Aphasiology, 29, 526-562. doi:10.1080/02687038.2014.985884

Kiran, S., Sandberg, C., \& Abbott, K. (2009). Treatment for lexical retrieval using abstract and concrete words in patients with aphasia: Effect of complexity. Aphasiology, 23, 835853. doi:10.1080/02687030802588866

Lundqvist, D., Flykt, A., \& Öhman, A. (1998). The Karolinska Directed Emotional Faces $K D E F$, CD ROM from Department of Clinical Neuroscience, Psychology section, Karolinska Institutet.

Renvall, K. \& Nickels, L. (2019). Using treatment to improve the production of emotive adjectives in aphasia: a single case study. Manuscript under review.

Wisenburn, B. \& Mahoney, K. (2009). A meta-analysis for word-finding treatments for aphasia. Aphasiology, 23, 1338-1352. doi: 10.1080/02687030902732745 


\title{
Investigating over-generalisation following word-retrieval treatment in Semantic Dementia
}

\author{
Lyndsey Nickels ${ }^{1,2}$, Sharon Savage ${ }^{2,3}$, Leonie Lampe ${ }^{1,2,4}$, Olivier Piguet ${ }^{2,5}$ \& John Hodges ${ }^{2,6}$ \\ ${ }^{1}$ Department of Cognitive Science, Macquarie University, Sydney, Australia \\ ${ }^{2}$ Australian Research Council Centre of Excellence in Cognition and its Disorders, Australia \\ ${ }^{3}$ Psychology department, University of Exeter Medical School, Exeter, UK \\ ${ }^{4}$ International Doctorate for Experimental Approaches to Language and Brain (IDEALAB), \\ Universities of Groningen (NL), Potsdam (DE), Trento (IT), Newcastle (UK) and Macquarie \\ University, Sydney (AU) \\ ${ }^{5}$ Brain 85 Mind Centre and School of Psychology, University of Sydney, Australia \\ ${ }^{6}$ Brain $\&$ Mind Centre and Clinical Medical School, University of Sydney, Australia
}

\section{Introduction}

Semantic Dementia (SD), the semantic variant of Primary Progressive Aphasia, is a form of frontotemporal dementia in which communication skills are progressively impaired through a loss of conceptual knowledge (Gorno-Tempini et al., 2011; Hodges, Patterson, Oxbury, \& Funnell, 1992). Impaired word retrieval is an early and striking feature of semantic dementia, as in other forms of progressive (and non-progressive) aphasia. Nevertheless, naming of treated items has been shown to improve with treatment (Carthery-Goulart et al., 2013; Croot, 2018; Jokel, Graham, Rochon, \& Leonard, 2014).

It is well known that word retrieval treatment rarely results in generalisation of improvement to untreated items. However, in semantic dementia there is also the concern that overgeneralisation may occur (Lambon Ralph \& Patterson, 2008; Mayberry et al., 2011) with treated labels misapplied to non-targets. It is possible that these errors could arise due to a loss of appreciation of the specificity of the label (e.g. that each element of a computer has its own name and is called a keyboard, monitor or mouse and not "a computer", e.g., Savage et al., 2015b). Alternatively, distinctions between concepts may no longer be apparent, leading to use of the same label for different concepts, for example calling a cat a "small dog", or all long thin vegetables "carrot" (e.g., Rogers et al., 2004). Finally, in earlier stages of the disease, an available related word may be used strategically in place of a target that is unavailable. Whatever the cause, it is clear that if treatment results in significant increases in these kinds of errors, it creates the potential for confusion and frustration in everyday communication.

Few studies have investigated the occurrence of overgeneralisation errors. By studying a series of participants with semantic dementia $(n=9)$ who had previously taken part in a lexical retrieval treatment programme we aimed to determine whether following treatment: 1) misuse of words increased; 2) error types changed; 3) clarity of communication reduced when target words were not retrieved.

\section{Methods}

Nine participants who met criteria for the semantic variant of Primary Progressive Aphasia (Gorno-Tempini et al., 2011) and had previously taken part in a word-retraining study run by FRONTIER (the Frontotemporal Dementia Research Group) in Sydney, Australia were included in the study. Eight of the nine participants were reported in Savage et al. (2015a). Each participant had a history of word finding difficulties for between three and nine years and showed marked naming deficits on standard tests when compared with age-matched controls. Comprehension and semantic deficits ranged from mild to severe, as did the degree of general cognitive impairment as measured on the Addenbrooke's Cognitive Examination-Revised 
(Mioshi, Dawson, Mitchell, Arnold, \& Hodges, 2006). The typical pattern of anterior and medial temporal lobe atrophy was observed in each case, with left greater than right atrophy in seven participants, and the reverse pattern in two participants.

For each participant, performance on trained and untrained words from final baseline and immediate post-intervention assessments was examined. Responses were: 1) coded to identify instances of misuse, where a word from the training list was used to refer to an untrained item; 2) assigned an error code, and, 3) scored for clarity, using a scale from -1 (clearly incorrect) through to +1 (correct).

\section{Results}

Naming accuracy significantly improved for trained words in all participants from baseline to immediate post-intervention (McNemar's Test, all $p<.005$; Savage et al., 2015a); untrained words mostly remained unchanged.

Does training encourage the misuse of treatment target words to non-target items?

Analyses for both misuse and repetition of treated words revealed no significant increase from baseline to post-intervention for any of the participants, either within the trained set or within untrained words (see Table 1). Further examination of the context of misuse revealed that in 34 of the 39 instances, the misused word was semantically related to the alternative item, suggestive of overgeneralising target words.

\section{Does the nature of errors change from pre-to post-treatment?}

While the rate of target naming attempts (versus non-target attempts, i.e. a description or omission) varied among individuals at baseline, no significant change occurred in the proportion of naming attempts of untrained words from pre- to post-intervention performance for the group overall (Wilcoxon, $p=.859$ ). However, the overall pattern of error response on the untrained words was statistically different across the two assessments (Cochran-Mantel-Haenszel test $2 \mathrm{MH}(5)=12.245, p=.032)$. Semantic errors and omissions continued to account for the bulk of errors across the time points ( $86 \%$ pre- $84 \%$ post-intervention), however, there was some increase in omissions $(42 \%$ to $50 \%$ ) with a relative reduction in semantic errors $(44 \%$ to $34 \%$ ). Individual error patterns remained highly similar across the two testing sessions for five of the nine participants (S2, P1, G1, J2, T4). In the remaining four, some changes were observed in the relative distribution of the errors, particularly for semantic errors versus omissions, but in only one case was this substantial (C1).

Was there any indication of negative impact of training on the clarity of responses?

While clarity scores increased from baseline to post-intervention for trained words (all $p<.038$ ), only one participant showed decline in clarity for untrained words (J1: $p=.038$ ), while two showed significantly improved clarity (S2: $p=.01 ; \mathrm{G} 1: p=.025)$.

\section{Discussion}

No negative impacts were observed following lexical retrieval treatment for untrained words. Specifically there was no significant increase in the misuse of trained vocabulary immediately after the 4-week treatment programme and the clarity of communication did not appear to be adversely affected. This study therefore provides further evidence of the benefit of these programs for patients with Semantic Dementia without any negative impact on untrained items. It is of particular note that this was the case even for participants with more severe semantic impairment. However, the items (both trained and untrained) were only included in the study when individuals retained some recognition of the object. This may have served to remove 
Table 1. Participant error patterns and clarity before and after intervention.

\begin{tabular}{|c|c|c|c|c|c|c|c|c|c|c|c|c|}
\hline \multirow[t]{2}{*}{ Participant } & \multicolumn{3}{|c|}{ Trained targets named using other trained words } & \multicolumn{3}{|c|}{ Untrained targets named using trained words } & \multicolumn{3}{|c|}{ Repetition errors per testing session } & \multicolumn{3}{|c|}{ Mean Clarity Score - Untrained } \\
\hline & BL & Post & Pre-post (exact p) & BL & Post & Pre-post (exact p) & BL & Post & Pre-post (exact p) & BL & $\begin{array}{l}\text { wor } \\
\text { Post }\end{array}$ & $\begin{array}{l}\text { Pre-post } \\
\text { (exact p) }\end{array}$ \\
\hline S1 & $0 / 32$ & $0 / 32$ & NA & $2 / 64$ & $3 / 64$ & 1.0 & $5 / 96$ & $2 / 96$ & .250 & .680 & .539 & .071 \\
\hline J1 & $2 / 30$ & $0 / 30$ & .50 & $1 / 57$ & $3 / 57$ & .50 & $2 / 87$ & $1 / 87$ & 1.0 & .544 & .430 & .029 \\
\hline S2 & $0 / 31$ & $0 / 31$ & NA & $1 / 66$ & $1 / 66$ & 1.0 & $1 / 97$ & $0 / 97$ & 1.0 & .295 & .462 & $.010^{*}$ \\
\hline P1 & $0 / 32$ & $0 / 32$ & NA & $4 / 65$ & $3 / 65$ & 1.0 & $2 / 97$ & $1 / 97$ & 1.0 & .308 & .346 & .272 \\
\hline B1 & $0 / 30$ & $0 / 30$ & NA & $1 / 65$ & $1 / 65$ & 1.0 & $3 / 95$ & $1 / 95$ & .500 & .469 & .508 & .475 \\
\hline G1 & $0 / 31$ & $1 / 31$ & 1.0 & $1 / 62$ & $2 / 62$ & 1.0 & $2 / 93$ & $2 / 93$ & 1.0 & .371 & .548 & $.013^{*}$ \\
\hline J2 & $0 / 25$ & $0 / 25$ & NA & $0 / 39$ & $0 / 39$ & NA & $0 / 64$ & $0 / 64$ & NA & .474 & .526 & .595 \\
\hline C1 & $1 / 38$ & $1 / 38$ & 1.0 & $3 / 72$ & $2 / 72$ & 1.0 & $4 / 110$ & $1 / 110$ & .375 & .597 & .611 & .839 \\
\hline T4 & $1 / 29$ & $0 / 29$ & 1.0 & $0 / 71$ & $5 / 71$ & .063 & $2 / 100$ & $5 / 100$ & .453 & .197 & .148 & .449 \\
\hline ALL & $4 / 278$ & $2 / 278$ & .285 & $13 / 561$ & $20 / 561$ & .116 & $21 / 839$ & $13 / 839$ & .176 & .433 & .453 & .515 \\
\hline
\end{tabular}

BL : baseline assessment; * significant change $(p<.05)$

the impact of semantic impairment by focusing in on vocabulary where some knowledge was retained. Consequently it remains possible that for items where conceptual knowledge is lost overgeneralisation may occur.

\section{References}

Carthery-Goulart, M., Silveira, A., Machado, T., Mansur, L., Parente, M., Senaha, M. L. H., ... Nitrini, R. (2013). Nonpharmacological interventions for cognitive impairments following primary progressive aphasia: a systematic review of the literature. Dementia $\&$ Neuropsychologia, 7(1), 122-131.

Croot, K. (2018). Treatment for Lexical Retrieval Impairments in Primary Progressive Aphasia: A Research Update with Implications for Clinical Practice. Seminars in Speech and Language, 39(3), 242-256. https://doi.org/10.1055/s-0038-1660783

Gorno-Tempini, M. L., Hillis, A. E., Weintraub, S., Kertesz, A., Mendez, M., Cappa, S. F., ... Grossman, M. (2011). Classification of primary progressive aphasia and its variants. Neurology, 76(11), 1006-1014. https://doi.org/10.1212/WNL.0b013e31821103e6

Hodges, J. R., Patterson, K., Oxbury, S., \& Funnell, E. (1992). Semantic dementia: Progressive fluent aphasia with temporal lobe atrophy. Brain, 115(6), 1783-1806. https://doi.org/10.1093/brain/115.6.1783

Jokel, R., Graham, N. L., Rochon, E., \& Leonard, C. (2014). Word retrieval therapies in primary progressive aphasia. Aphasiology, 28(8-9), 1038-1068.

https://doi.org/10.1080/02687038.2014.899306

Lambon Ralph, M. A., \& Patterson, K. (2008). Generalization and differentiation in semantic memory: insights from semantic dementia. Annals of the New York Academy of Sciences, 1124, 61-76. https://doi.org/10.1196/annals.1440.006

Mayberry, E. J., Sage, K., Ehsan, S., \& Lambon Ralph, M. A. (2011). Relearning in semantic dementia reflects contributions from both medial temporal lobe episodic and degraded neocortical semantic systems: Evidence in support of the complementary learning systems theory. Neuropsychologia, 49(13), 3591-3598.

Rogers, T. T., Lambon Ralph, M. A., Garrard, P., Bozeat, S., McClelland, J. L., Hodges, J. R., \& Patterson, K. (2004). Structure and Deterioration of Semantic Memory: A Neuropsychological and Computational Investigation. Psychological Review, 111(1), 205235. https://doi.org/10.1037/0033-295X.111.1.205

Savage, S. A., Piguet, O., \& Hodges, J. R. (2015a). Cognitive Intervention in Semantic Dementia: Maintaining Words Over Time. Alzheimer Disease and Associated Disorders, 29(1), 55-62. https://doi.org/10.1097/WAD.0000000000000053

Savage, S. A., Piguet, O., \& Hodges, J. R. (2015b). 'Knowing What You Don't Know': 
Language Insight in Semantic Dementia. Journal of Alzheimer's Disease: JAD, 46(1), 187-198. https://doi.org/10.3233/JAD-142703 


\title{
Strategy, cognition, and communication partners: Maximizing treatment impact in progressive aphasia and Alzheimer's disease
}

\author{
Ashleigh Beales ${ }^{1}$, Anne Whitworth ${ }^{1}$, Jade Cartwright ${ }^{1}$, Peter K Panegyres ${ }^{2}$, \& Robert T \\ Kane $^{3}$ \\ ${ }^{1}$ School of Occupational Therapy, Social Work and Speech Pathology, Curtin University, \\ Perth, Western Australia, Australia \\ ${ }^{2}$ Neurodegenerative Disorders Research, West Perth, Western Australia, Australia \\ ${ }^{3}$ School of Psychology, Curtin University, Perth, Western Australia, Australia
}

\section{Introduction}

While the growing body of treatment evidence for people with progressive aphasia is testimony to the transferability of lexical retrieval interventions effective with post-stroke aphasia (Croot, 2018), the diversity of therapy approaches applied does not allow a clear understanding as to why they have been successful and whether critical ingredients are at play. In the primary progressive aphasia (PPA) literature, treatment gains have been reported following self-cueing strategies (e.g. Beales, Cartwright, Whitworth, \& Panegyres, 2016) as well as multi-level discourse interventions (e.g. Whitworth et al., 2017). In comparison to PPA, the diversity of therapies that have been reported in the Alzheimer's disease (AD) literature is more limited and largely focused on memory-based therapies. While speech and language outcomes during and following intervention have been a dominant focus in this literature, understanding the nonlinguistic cognitive processes that contribute to and interact with these is likely also to be critical. In a review of PPA and AD lexical retreival interventions, autobiographical memory and episodic memory were the only nonlinguistic cognitive processes found to have been considered in the planning of intervention or interpretation of intervention outcomes (Beales, Whitworth, \& Cartwright, 2018). Other nonlinguistic cognitive functions such as attention, working memory, and visual memory are yet to be explored within the PPA and AD literature, however, are likely to impact an individual's ability to engage in therapy, as well as their capacity to take on strategies. In a recent study, Grasso, Shuster, and Henry (2017) administered a lexical retrieval intervention, which comprised a clinician- and caregiver-administered treatment phase, to two individuals with progressive lexical impairments. Involvement in intervention was positively received by family members, specifically, improved ability to guide descriptions during naming difficulty and reduced frustration during communicative interactions when learned strategies were employed, were reported by caregivers (Grasso et al., 2017). These preliminary findings of positive responses to participation in communication intervention affirm the importance of further understanding and maximizing the role of communication partners.

Aim

This study investigated a cognitive-linguistic self-cueing intervention trained to explicitly facilitate generalization through the inclusion of communication partners and practice at the discourse level. Generalization of intervention to untreated items and to discourse production, and quantitative measures of perceived change in communicative ability, were examined.

\section{Methods}

\section{Participants}

Twelve participants, aged between 55 and 86 years $(M=65.5)$, with progressive lexical impairments were recruited to the study. Eight participants had a diagnosis of PPA (four semantic variant and four logopenic variant) and four participants had a diagnosis of AD. Family members were also invited to participate. 


\section{Protocol}

A multiple baseline across-behaviours design was used in which participants acted as their own control. Three sets of baseline assessments were conducted prior to intervention and then repeated again at one-week (post-intervention) and six-weeks (maintenance) post-intervention to examine both treatment effectiveness and generalization of the intervention. The intervention was a language production treatment that incorporated cognitive scaffolds specific to each participant to train a self-cueing strategy to facilitate lexical access. In line with recommendations of previous research, the intervention involved explicit attempts to facilitate generalization through practice of this strategy at the connected speech level and working with communication partners, both of which are evaluated in the study. The intervention protocol expanded on the self-cueing strategy, reported in Beales et al. (2016) and which had introduced autobiographical memory cues to semantic, phonological, and orthographical domains, by incorporating individualized cognitive scaffolding for attention, working memory, verbal episodic memory, and visual memory based on participants' pre-intervention neuropsychological assessment.

\section{Results}

Evidence of both treatment effects and generalization to untreated items was found. Improved picture naming of treated and untreated items was found across all word types, i.e. nouns, verbs, and adjectives, with patterns varying for diagnostic groups. Limited evidence was found to indicate that benefits of intervention extended to changes in communicative informativeness and efficiency in discourse, or communicative ability as perceived by participants and their family members. The heterogeneity of nonlinguistic cognitive profiles called for the implementation of cognitive scaffolding during intervention that was tailored to the individual. No changes in cognitive measures were found post-intervention. Treatment effects and generalization to untreated items were generally maintained at follow-up testing.

\section{Discussion}

This study supports a growing body of evidence, which demonstrates the effectiveness of lexical retrieval interventions for individuals with PPA, and provides promising new directions. Uniquely, this study provides evidence that individuals with AD can also benefit from an impairment-based self-cueing intervention. The presence of consistent and significant generalization to untreated words across all diagnostic groups was unexpected and contrasts with the post-stroke literature where treatment effects have been, more frequently than not, itemspecific. Further investigation is warranted to examine the effects of cognitive scaffolding, comparing this approach to active training of these functions. By considering deficits beyond linguistic abilities in intervention design, such research will innovate more holistic speech-language management of clients with progressive lexical impairments.

\section{References}

Beales, A., Cartwright, J., Whitworth, A., \& Panegyres, P. K. (2016). Exploring generalization processes following lexical retrieval intervention in primary progressive aphasia. International journal of speech-language pathology, 18(3), 299-314.

Beales, A., Whitworth, A., \& Cartwright, J. (2018). A review of lexical retrieval intervention in primary progressive aphasia and Alzheimer's disease: mechanisms of change, generalization, and cognition. Aphasiology, 1-28.

Croot, K. (2018). Treatment for lexical retrieval impairments in primary progressive aphasia: A research update with implications for clinical practice. Seminars in Speech and Language, 39(3), 242-256. 
Grasso, S. M., Shuster, K. M., \& Henry, M. L. (2017). Comparing the effects of clinician and caregiver-administered lexical retrieval training for progressive anomia. Neuropsychological rehabilitation, 1-30.

Whitworth, A., Cartwright, J., Beales, A., Leitão, S., Panegyres, P. K., \& Kane, R. (2017). Taking words to a new level: a preliminary investigation of discourse intervention in primary progressive aphasia. Aphasiology, 1-26. 


\title{
Effect of Type of Language Therapy on Language Improvement in Patients with Post-Stroke Aphasia
}

\author{
Jasmina Vuksanović ${ }^{1}$, Tanja Milovanović ${ }^{2}$, Ljubica Konstantinoviće,3, Saša R. Filipović ${ }^{1}$ \\ ${ }^{1}$ University of Belgrade, Institute for Medical Research, Dr Subotica 4, Belgrade, Serbia \\ ${ }^{2}$ Klinika za Rehabilitaciju "Dr Miroslav Zotović, Sokobanjska 13, Belgrade, Serbia \\ ${ }^{3}$ University of Belgrade, Faculty of Medicine (Rehabilitation), Dr Subotica 8, Belgrade, Serbia
}

\section{Introduction}

The vast majority of patients require speech and language therapy of some kind in order to recover specific language skills affected by damage of the brain and to develop strategies for improvement of overall communication in a variety of situations (Basso, 2010; Basso and Macis, 2011). Stimulation aphasia therapy (SAT), sometimes also referred as conventional (or classic) aphasia therapy, is one of the most frequently used therapeutic techniques. It is based on an idea that language is not lost but cannot be accessed because of cerebral damage. Common to all traditional therapy approaches, including SAT, is that communicative methods allow for all modes of communication to be used, i.e., communicative methods are not constraint to the language mode, but include both verbal and non-verbal means. The person with aphasia is encouraged to use all their intact communication abilities, which are often mainly non-verbal, in order to optimize communication. Another approach to language therapy, the Constraintinduced (language) aphasia therapy (CIAT), has been developed as potentially more efficient aphasia therapy method. CIAT is based on the following learning principles: prevention of compensatory communication (constraint), massed practice (maximization of quantity and frequency of language therapy), and shaping (induction) of verbal behaviour (Pulvermuller et al. 2005, Pulvermuller and Berthier 2008).

In this study we examined the comparative efficacy of the CIAT and the SAT approach on expressive language ability. We focused on naming ability and spontaneous sentence production, with the intensity of therapy controlled and matched between therapy methods. In order to reduce the influence of various confounding variables and to shed more light onto the interplay between the two CIAT principles, massed practice and constrained use of the language modality, the experimental study was designed to be a crossover research (Maclure 1991).

\section{Methods}

\section{Procedure}

Two successive 4-week blocks of intense (1-hour, 5 days a week) aphasia therapy of each therapy program were delivered in a randomized within-subject crossover design -one therapy block consisted of SAT, another of CIAT. Twenty consecutive patients, up to a year after stroke, were randomly assigned to either have SAT followed by CIAT (S1C2 group), or to have CIAT followed by SAT (C1S2 group). Measurements of naming (Boston Naming Test) and spontaneous sentence production (Cookie Theft Picture description task) were carried at the baseline, following the first therapy block, following the second therapy block, and four-weeks following the last therapy block.

\section{Subjects}

A total of 20 consecutive right-handed patients with non-fluent post-stroke aphasia of up to 1 year duration were randomly assigned to two treatment groups. One group had SAT first followed by CIAT (S1C2 group), while another had a reversed order of the therapies, CIAT first followed by SAT (C1S2 group). For all participants, diagnosis of post-stroke aphasia was made by neurologists and confirmed by speech therapists based on their assessment, which included 
the Boston Diagnostic Aphasia Examination (BDAE; Goodglass and Kaplan 1983). The participants, and their family members when it was required due to communication difficulties, gave their informed consent for the participation in the study. The study was conducted according to the Declaration of Helsinki. The study was approved by the institution ethics committee.

\section{Results}

Both groups of patients significantly improved in all variables of expressive language skills; the improvement was maintained 1 month post-treatment. However, patients who started with CIAT and continued with SAT (C1S2 group) tended to have higher final improvement than the patients who started with SAT and continued with CIAT (S1C2 group). This was particularly pronounced for naming recovery. Moreover, when CIAT was the first therapy (the C1S2 group) the improvement in naming achieved following CIAT significantly exceeded the improvements achieved following SAT, not only in the same group (when SAT was the second), but also in $\mathrm{S} 1 \mathrm{C} 2$ group (when SAT was the first); in addition, the improvement in naming following CIAT in $\mathrm{C} 1 \mathrm{~S} 2$ group exceeded the one in $\mathrm{S} 1 \mathrm{C} 2$ group too. Similarly, the improvement in total number of sentences produced in response to the picture was quite clear and significant following each of the therapies, the CIAT and the SAT, in C1S2 group (when CIAT was the first), while in the $\mathrm{S} 1 \mathrm{C} 2$ group the improvement following either type of therapy was much more modest and not significant.

\section{Discussion}

Results suggest that the maximization of quantity and frequency of language therapy have a significant impact on the improvement of expressive language ability. Moreover, if practised early in aphasia therapy, the constrained usage of the language modality, as practised in CIAT, confers an additional benefit to massed practice, particularly on naming ability.

\section{References}

Basso, A. (2010). Natural conversation: a treatment for severe aphasia. Aphasiology, 24, 466479.

Basso, A., and Macis, M. (2011). Therapy efficacy in chronic aphasia. Behavioural Neurology, 24, 317-325.

Goodglass, H. and Kaplan, E. (1983). The Assessment of Aphasia and Related Disorders (Philadelphia, PA: Lea \& Febiger).

Maclure, M. (1991). The case-crossover design: a method for studying transient effects on the risk of acute events. American Journal of Epidemiology, 133(2), 144-153.

Pulvermuller , F. and Berthier, M. L. (2008). Aphasia therapy on a neuroscience basis. Aphasiology, 22, 563-599.

Pulvermuller, F., Hauk, O., Zohsel, K., Neininger, B. and Mohr, B. (2005). Therapy-related reorganization of language in both hemispheres of patients with chronic aphasia. Neuroimage, 28, 481-489. 


\section{Poster Session III}




\title{
Communicating and fixing communication breakdowns with people with aphasia: Speech-language therapists' (SLTs) and caregivers' perspectives
}

\author{
Özlem Ŏguz \\ Üsküdar University
}

\begin{abstract}
Introduction
The effects of aphasia range from difficulty to talk, understand, write and read to depression, frustration, divorces and unemployment. The difficulties that are experienced after damage to the language-specific area in left hemisphere affect three sides: the people with aphasia, the caregiver who is mostly spouse and the SLTs. In the light of studies that mostly focus on assessment procedures, therapy approaches and language characteristics, some other studies examine the behaviors that accompany with language difficulties. These studies have demonstrated that crying, pettishness, giving up and being dependent on caregivers' expressions are just some of the behaviors. The difficulties that is experienced by caregivers make the process of taking care of the person with aphasia harder and stressful than it should be because of increased dependency, social isolation and other negative behaviors. Communication with people with aphasia is already a challenge for the caregivers as a result of language barrier. In addition to language difficulties, managing behavioral problems and at the same time trying to provide emotional and homework support is backbreaking for the caregivers. SLTs focus on language tasks and prognosis in the rehabilitation process. Like caregivers, they also experience the behavioral problems and try to make the process smooth. However both sides should turn their 'improving language interest' into widening rehabilitation horizons which includes dealing with the problematic behaviors with training and education.
\end{abstract}

\section{Methods}

The purpose of the current study is to explore the behavioral problems which accompany with language difficulties after aphasia and repair strategies of communication breakdowns of SLTs and caregivers' through responses. Some situations are given to the participants to choose according to the occurrence in everyday life and inside clinic in the first. Following the first part, the participant are supposed to say or write about how they handle the situation when they experience each of it. In the end, implications about frequently encountered problems and handling the process are intended to make.

\section{Material}

In the present study, a questionnaire that have two parts are developed to identify accompanying behaviors and strategies/responses of SLTs after some research and interviews with SLTs. Participants are answered to questions about demographics and experience of years in the profession or with the person with aphasia. In the first part, there are 17 situations including crying or laughing out of sudden, losing motivation, swearing, commit violence, being dependent on care giver, expecting care giver to complete their sentence, giving up, not taking the SLT or the activities serious, talking nonstop and etc. The second part includes open ended questions which ask the participants what they do when they encounter with the given situations.

\section{Participants}

36 SLTs and 16 caregivers participated in the study. The SLTs had experiences ranging from one year to more than ten years. The caregivers including mostly spouses, daughter and daughter in law- spent time with the person with aphasia for mostly 1-4 years. The participants were given options to choose and they answered the open-ended questions via orally or writing. 
Table 1. Participant demographics

\begin{tabular}{lllll}
\hline & SLTs & \multicolumn{3}{l}{ Caregivers } \\
\cline { 2 - 5 } & $\%$ & $\mathrm{~N}$ & $\%$ & $\mathrm{~N}$ \\
\hline Sex & $72,2 \%$ & 26 & $75 \%$ & 14 \\
Female & $27,8 \%$ & 10 & $25 \%$ & 4 \\
Male & $55,6 \%$ & 21 & $12,5 \%$ & 2 \\
\hline Age & $27,8 \%$ & 10 & $12,5 \%$ & 2 \\
$22-30$ & $11,1 \%$ & 4 & $43,8 \%$ & 7 \\
$31-40$ & $5,6 \%$ & 2 & $31,2 \%$ & 5 \\
$41-50$ & & & & \\
$51+$ & $27,8 \%$ & 10 & $31,2 \%$ & 5 \\
\hline Experience & $33,3 \%$ & 12 & $43,8 \%$ & 7 \\
$0-1 \quad$ years & $19,4 \%$ & 7 & $12,5 \%$ & 2 \\
$2-4$ years & $8,4 \%$ & 3 & $6,3 \%$ & 1 \\
$5-7$ years & $11,1 \%$ & 4 & $6,3 \%$ & 1 \\
$7-10$ years & & & & \\
+10 years & $50 \%$ & 18 & - & - \\
\hline Working place & $55,6 \%$ & 20 & - & - \\
Private clinic & $8,3 \%$ & 3 & - & - \\
Rehabilitation center & $25 \%$ & 9 & - & - \\
Hospital & $11,1 \%$ & 4 & - & - \\
University (Academic personel) & & & \\
Other & & & & \\
\hline
\end{tabular}

\section{Results}

The both participant groups have stated that the most often experienced behaviour was 'frustration of the person with aphasia feels like he/she hasn't been understood' and 'giving up on saying something or not completing the sentence' follows. The responses and repair strategies change according to SLTs and caregivers. Most of the caregivers let the person with aphasia continue the behavior when they get angry or too talkative while SLTs intervene and try to replace the behavior with some other desired one. In terms of handling self-despair and crying behavior of the person with aphasia, both SLTs and caregivers stated that the try to cheer the person up. While the caregivers do it by talking and motivating, the SLTs prefer changing the activity or telling motivation stories and trying different other ways which is surprisingly innovator such as listening favorite songs.

\section{Discussion}

The responses do not change in very big scales but the results show that training of the care givers and letting them be in the rehabilitation process needs to be done. Managing communication difficulties and using strategies vary as in other research findings (Johansson et all, 2012; Bakas et all. 2006). With joint training of partner and SLTs in the field of handgling behavioral problems could lead not only more effective and joyful but also less stressful and exhausting process.

\section{References}

American Speech-Language-Hearing Association. Family Adjustment to Aphasia. https://www.asha.org/public/speech/disorders/FamilyAdjustmentAphasia.html 09.04.2019

Bakas, T., Kroenke, K., Plue, L.D. Perkins, S.M. \& Williams, L.S. (2006). Outcomes Among Family Caregivers of Aphasic Versus Nonaphasic Stroke Survivors. Rehabilitation Nursing. 31 (1): 33-42.

Dobkin BH. (2016). Rehabilitation and recovery of the patient with stroke. In: Grotta JC, 
Albers GW, Broderick JP, et al, eds. Stroke: Pathophysiology, Diagnosis, and Management.

Johansson , M.B., Carlsson, M., Östberg, P. \& Sonnander, K. (2012) Communication changes and SLP services according to significant others of persons with aphasia, Aphasiology, 26:8, 1005-1028.

Long, A.,Hesketh, A. \& Bowen, A. (2009). Communication outcome after stroke: a new measure of the carer's perspective. Clinical Rehabilitation. 23: 846-856.

Page S. (2013). Guide to living with aphasia. Arch Phys Med Rehabil. 94(8):1643-1644. 


\title{
Verbal and nonverbal fluency in presymptomatic carriers of the Huntington's disease gene
}

\author{
Šešok $\mathrm{S}^{1}$, Bolle $\mathrm{N}^{2}$, Kobal J $\mathrm{J}^{3}$ \\ ${ }^{1}$ Department of Neurorehabilitation, Department of Neurology, University medical center \\ Ljubljana, Slovenia \\ ${ }^{2}$ Outpatient Rehabilitation Service, University Rehabilitation Institute, Republic of Slovenia, \\ Slovenia \\ ${ }^{3}$ Department of Vascular Neurology and Intensive Neurologic Therapy, Department of \\ Neurology, University Medical Centre Ljubljana, Slovenia
}

\section{Introduction}

Huntington's disease (HD) is a neurodegenerative inherited disorder caused by an increased number of CAG repeats in the IT15 gene of the 4th chromosome. The product of mutation is pathologic protein huntingtin, which is toxic for many tissues, but most highly expressed in the central nervous system (CNS) (1). The availability of genetic analysis for the diagnosis of HD now allows for a careful study of clinically presymptomatic HD gene carriers. HD clinical onset is most readily defined with the onset of motor symptoms e.g. chorea (2). Research so far has suggested that cognitive dysfunction in HD is the earliest clinical symptom and may start years before the clinical disease onset (3). The aim of this study was to investigate which deficits already occur in clinically presymptomatic HD-gene carriers and which specific cognitive functions are impaired compared to control group of healthy individuals of the same age, sex and education distribution. It has been suggested so far that cognitive impairments relate to a common deficit in inhibitory control mechanisms (breakdown in the mechanisms of response selection), under the control of striatofrontal structures. However, still it remains unclear which part of striatofrontal loop is affected initially (4.). A recent MR study found early affection of cortical neurons and their axons (5). It is therefore not necessary that degeneration process starts in basal ganglia; they might merely be the most vulnerable tissue where atrophy is first to be seen.

\section{Methods}

Twenty presymptomatic gene carriers (10 male, mean age 34 years) which were free of clinical movement disorder, affective disorders and/or psychotic symptoms were enrolled in our study together with a matched control group of healthy individuals. Cognitive functions were evaluated using a broad neuropsychological assessment. The tests which were found most sensitive previously were used for evaluation as well asother verbal and nonverbal tests were added. Among the cognitive functions evaluation of executive functions was emphasized. The apparatus is presented as follows:

\section{Statistics}

Normality of distribution was assessed using the Shapiro-Wilks test. The variance equality assumption was verified with the Levene's test. Group differences were then examined using the t-test for independent samples and the Kolmogorov-Smirnov Z test, accordingly. Significance level for all tests was set at $p<0.05$.

\section{Results}

Neuropsychological findings showed that the group of asymptomatic gene carriers displayed significantly lower scores on some executive tests. The scores are presented on table 1.

We found a pattern of results which was partly expected, according to previous reports e.g. significantly different SDMT total score and Stroop color-word total score (7). However subsets 
Neuropsychological assessment administered to study participants

\begin{tabular}{|c|c|}
\hline Neuropsychological test & Cognitive domains assessed \\
\hline SDMT & $\begin{array}{l}\text { psychomotor speed, sustained attention, ability to integrate different verbal } \\
\text { and perceptual nonverbal cognitive processes }\end{array}$ \\
\hline Stroop & $\begin{array}{l}\text { cognitive flexibility, inhibition, resistance to interference, speed of cognitive } \\
\text { processing }\end{array}$ \\
\hline CTMT & attention, visual scanning, motor speed, set-shifting, sequencing abilities \\
\hline $\mathrm{d} 2$ & $\begin{array}{l}\text { selective and sustained attention, attentional and inhibitory control, coordina- } \\
\text { tion of speed and accuracy performance }\end{array}$ \\
\hline VF & verbal fluency \\
\hline WCST & cognitive flexibility, sensitive to prefrontal cortical function \\
\hline CT-cv & $\begin{array}{l}\text { nonverbal cognitive flexibility and ability to alter nonverbal problem-solving } \\
\text { strategies }\end{array}$ \\
\hline RFFT & $\begin{array}{l}\text { nonverbal capacity for fluid and divergent thinking, ability to flexibly shift } \\
\text { cognitive set }\end{array}$ \\
\hline TOL & $\begin{array}{l}\text { executive problem solving and planning, behavioral inhibiton, rule-governed } \\
\text { behavior }\end{array}$ \\
\hline
\end{tabular}

SDMT - Symbol Digit Modalities Test, Stroop - Stroop Color and Word Test, CTMT - Comprehensive

Trail-Making Test, d2 - d2 test of Attention, VF - Verbal Fluency, WCST - Wisconsin Card Sorting Test,

CT-cv - Category test (computer version), RFFT - Ruff Figural Fluency Test, Test TOL - Tower of London

of other verbal and nonverbal tests, e.g. TOL rule violations score, CTMT initial score, and RFFT unique design score were also found sensitive.

\section{Discussion}

The aim of our study was to investigate which deficits occur in clinically preymptomatic HDgene carriers and which specific cognitive functions are impaired. Our hypothesis was based on previous research (6) revealing that cognitive dysfunction in HD is mostly located in the sphere of executive functions. Psychomotor slowing observed in HD subjects may however add significantly to poorer results (4) Our results are partly in line with results of previous studies. The SDMT, Stroop and trail making test were found to demonstrate psychomotor slowing most significantly (4). SDMT test was found to correlate significantly to degree of caudate nucleus atrophy, while Stroop total color-word score significantly correlates to the age at disease onset (7). However, our study encompassed multiple neuropsychological tests and we found other cognitive deficits in clinicaly presymptomatic HD gene carriers involving TOL rule violation score and RFFT unique design score impairment. While TOL test is oriented mostly towards executive problem solving and planning RFFT test is oriented towards nonverbal capacity for fluid and divergent thinking and ability to flexibly shift cognitive set. TOL rule violations score has also been found sensitive in other disorders. RFFT has been used less extensively by now and according to our information not in HD clinically presymptomatic gene carriers $(8,9)$. Design fluency tests, among them RFFT, have been used in frontal lobe pathology research, showing it disrupts the ability to switch between many possible strategies to optimize response production while also avoiding repetition. (10,11,12). TOL and RFFT are both tests that require following restrictions and adequate impulse control and rule violation errors have been shown to be associated with right lateral prefrontal atrophy in neurodegenerative disease (13). Both tests are therefore oriented towards executive functioning but neither was used in HD patients extensively. One of such contributions using TOL test concludes that striatal pathology does not necessarily contribute to executive dysfunction in Huntington disease (14). According to our evidence, verbal and nonverbal tasks represented with verbal fluency and RFFT test are affected significantly as well as several other verbal and nonverbal executive tests. This fits into recent neuroimaging findings showing that white matter is affected according to pattern of cortical degeneration (5).

In short, we showed that cognitive dysfunction in presymptomatic HD gene carriers is complex, 
it encompasses verbal as well as nonverbal domains which were understudied so far (7). Our findings are in line to recent neuroimaging studies revealing early cortical and white matter dysfunction. Such pattern of affection reveals possibility of new therapeutic targets in HD.

Table 1. Results of executive function measures for ANG and healthy controls, with descriptive statistc, significance level and effect size. Significance level was set at: $p<0.05$ (independent t-test and Kolmogorov-Smirnov Z test)

\begin{tabular}{|c|c|c|c|c|c|c|}
\hline \multirow[t]{2}{*}{ Measure } & \multicolumn{2}{|c|}{$\begin{array}{l}\text { Asymptomatic gene } \\
\text { carriers }\end{array}$} & \multicolumn{2}{|c|}{ Healthy controls } & \multirow[t]{2}{*}{$p$} & \multirow[t]{2}{*}{$r$} \\
\hline & $M$ & $S D$ & $M$ & $S D$ & & \\
\hline SDMT total score & 46,12 & 9,94 & 59,00 & 9,79 & 005 & ,56 \\
\hline STROOP word score & 95,41 & 11,02 & 102,82 & 7,79 & 040 & ,41 \\
\hline STROOP color score & 68,53 & 12,98 & 77,41 & 10,29 & 017 & ,36 \\
\hline STROOP color-word total score & 40,41 & 11,10 & 48,53 & 7,05 & ,008 & ,41 \\
\hline STROOP interference total score & 1,26 & 7,13 & 7,17 & 12,68 & 109 & 35 \\
\hline CTMT1 time (seconds) & 44,82 & 15,16 & 33,24 & 8,31 & ,004 & ,41 \\
\hline CTMT2 time (seconds) & 40,59 & 15,40 & 32,24 & 9,91 & 016 & ,47 \\
\hline CTMT3 time (seconds) & 39,29 & 12,27 & 33,65 & 8,91 & 067 & ,26 \\
\hline CTMT4 time (seconds) & 45,59 & 18,35 & 33,94 & 7,64 & 020 & ,47 \\
\hline CTMT5 time (seconds) & 74,18 & 32,83 & 53,18 & 16,53 & ,020 & ,47 \\
\hline $\mathrm{d} 2$ total number & 351,12 & 93,69 & 419,76 & 84,09 & 016 & ,37 \\
\hline d2 errors & 19,59 & 18,16 & 16,18 & 11,54 & ,500 & 12 \\
\hline $\mathrm{d} 2$ total number errors & 331,29 & 98,15 & 403,59 & 82,37 & 013 & ,38 \\
\hline d2 concentration performance & 129,94 & 46,31 & 160,00 & 33,27 & 018 & ,36 \\
\hline D2 FR & 14,65 & 6,07 & 13,71 & 4,5 & 306 & \\
\hline VF & 22,29 & 3,60 & 27,18 & 8,41 & 036 & ,41 \\
\hline WCST categories completed & 5,53 & 94 & 5,82 & 39 & 242 & 17 \\
\hline WCST trials to complete 1st category & 13,41 & 4,18 & 15,94 & 7,47 & ,226 & 17 \\
\hline WCST failure to maintain set & ,71 & 1,10 & ,76 & 97 & ,500 & 06 \\
\hline WCST learning to learn & ,77 & 4,78 & 1,51 & 3,56 & ,500 & 12 \\
\hline WCST total errors & 22,06 & 16,35 & 22,53 & 15,07 & 336 & 24 \\
\hline WCST perseverative responses & 12,94 & 10,74 & 11,18 & 5,89 & 180 & 29 \\
\hline WCST perseverative errors & 11,59 & 8,99 & 10,24 & 5,17 & 174 & 29 \\
\hline WCST nonperseverative errors & 10,47 & 8,19 & 12,29 & 10,37 & 319 & ,24 \\
\hline WCST conceptual level responses & 65,47 & 5,69 & 69,71 & 7,02 & ,031 & ,32 \\
\hline CT-CV errors & 53,29 & 25,32 & 37,12 & 20,01 & 052 & 29 \\
\hline RFFT unique design & 82,76 & 28,95 & 105,47 & 24,34 & 010 & ,40 \\
\hline RFFT errors & 17 & 19 & ,06 & ,06 & 157 & 41 \\
\hline TOL total move score & 40,18 & 26,66 & 34,29 & 14,22 & 351 & 29 \\
\hline TOL total correct score & 4,00 & 2,40 & 3,88 & 2,03 & 439 & 03 \\
\hline TOL total rule violations score & ,76 & 1,15 & ,00 & ,00 & 002 & ,47 \\
\hline TOL total time violations score & 1,12 & 1,45 &, 53 & ,94 & 168 & 18 \\
\hline TOL total initiation time & 52,59 & 34,12 & 48,82 & 29,50 & 474 & 17 \\
\hline TOL total execution time & 226,76 & 104,52 & 194,41 & 74,91 & ,482 & 18 \\
\hline TOL total problem solving time & 285,41 & 126,75 & 246,29 & 89,99 & 154 & 18 \\
\hline
\end{tabular}




\section{References}

1 McColgan P, Tabrizi SJ. Huntington's disease: a clinical review. Eur J Neurol. 2018; 25: 24-34. doi: 10.1111/ene.13413.

2 Kremer B. Clinical neurology of Huntington's disease. In: Bates G, Harper P.Jones L (eds). Huntington's disease. Oxford university press, Oxford, 2002: 28-61.

3 Paulsen JS, Langbehn DR, Stout JC, et al. Detection of Huntington's disease decades before diagnosis: the Predict-HD study. J Neurol Neurosurg Psychiatry. 2008;79:874-80.

4 David Craufurd and Julie Snowden. Neuropsychological and neuropsychiatric aspects of Huntington disease. In Bates G, Harper P.Jones L.(eds) Huntington's disease. Oxford university press, Oxford, 2002: 62-94.

5 Rosas HD, Wilkens P, Salat DH, et al. Complex spatial and temporally defined myelin and axonal degeneration in Huntington disease. Neuroimage Clin. 2018; 20: 236-242. doi: 10.1016/j.nicl.2018.01.029.

6 Šešok S, Bolle N, Kobal J, Bucik V, Vodušek DB. Cognitive function in early clinical phase huntington disease after rivastigmine treatment. Psychiatr Danub. 2014; 26: 239-48.

7 Paulsen JS, Long JD, Ross CA, et al. PREDICT-HD Investigators and Coordinators of the Huntington Study Group. Prediction of manifest Huntington's disease with clinical and imaging measures: a prospective observational study. Lancet Neurol. 2014 ;13(12):1193201. doi: 10.1016/S1474-4422(14)70238-8.

8 Riccio CA, Wolfe ME, Romine C, Davis B, Sullivan JR. The Tower of London and neuropsychological assessment of ADHD in adults. Arch Clin Neuropsychol. 2004 ;19: 661-71.

9 Ruff RM, Light RH, Evans RW. The Ruff Figural Fluency Test: A normative study with adults. Dev Neu-ropsychol. 1987; 3: 37-51.

10 Ruff RM, Allen CC, Farrow CE, et al Figural Fluency: Differential Impairment in Patients with Left Versus Right Frontal Lobe Lesions. Archivesof Clinical Neuropsychology, 1994;. 9: 41-55.

11 Jones-Gotman M, Milner B. Design Fluency: The Invention of Nonsense Drawings After Focal Cortical Lesions. Neuropsychologia. 1977; 15: 653 -674.

12 Baldo JV, Shimamura AP, Delis DC, et al. Verbal and design fluency in patients with frontal lobe lesions. Journal of the International Neuropsychological Society 2001; 7: 586-596.

13 Possin, KL, Brambati, SM, Rosen, HJ et al, 'Rule violation errors are associated with right lateral prefrontal cortex atrophy in neurodegenerative disease', Journal of the International Neuropsychological Society 2009; 15: 354.

14 Mörkl S, Müller NJ, Blesl C, et al. Problem solving, impulse control and planning in patients with early- and late-stage Huntington's disease. Eur Arch Psychiatry Clin Neurosci. 2016; 266::663-71. doi: 10.1007/s00406-016-0707-4. 


\title{
Exploring Phonological Deficits in French speakers with Acute stroke Aphasia: A Preliminary Study
}

\author{
Typhanie PRINCE \\ University Training Center for Speech-Language Pathology, University of Nantes, UMR 1253, \\ IBrain, INSERM, TOURS, FRANCE
}

\begin{abstract}
Introduction
Clinical-linguistic data on the phonological skills of people with aphasia (PWA) from several languages such as French, Spanish, Italian or Dutch reveal that PWA produce phonological paraphasias which affect the segmental content and/or the syllabic structures (Den Ouden and Bastiaanse, 2003; Laganaro, 2005, 2014; Nespoulous, Baqué, Rosas, Marczyck \& Estrada, 2013; Prince, 2017, 2018; Romani et al., 2011). However, syllable level has only been taken into account belatedly by neuropsycholinguistic models (Buckingham \& Buckingham, 2015; Den Ouden \& Bastiaanse, 2003; Levelt \& al. 1999), yet, its role is fundamental as evidenced by the phonological paraphasias. Due to phonological deficits, word structures become inaccessible leading PWA to develop word finding strategies like "repair strategies". These repair strategies reflect the organization of phonological units and their analyses highlight the syllable level and the syllable structures.
\end{abstract}

According to several studies (Goldrick \& Rapp, 2007; Laganaro, 2008, 2014; Laganaro \& Zimmermann, 2010), four main variables seem to be correlated with the production of phonological errors: lexical frequency, word length, lexicality and frequency of infra-lexical units. Phonological transformations are more important for low frequency infra-lexical units (the segments but also the syllables). Interestingly, PWA who have phonological and/or articulatory motor deficits produce more accurately syllables with high frequency than those with low frequency (Laganaro, 2014). Consequently, these studies suggest the involvement of the syllable frequency in the production of transformations. Moreover, high frequency syllables are also, from an articulatory point of view, the least complex. Syllable structures such as CV are less involved than CCV or CVC structures in phonological transformations. Because they are more difficult to test, complex syllabic structures are actually rarely taken into account by studies. However, could the syllable frequency effect be an artefact of the complexity effect? Wilshire \& Nespoulous (2003) examined conduction aphasia patients with a phonological deficit and found no effect of syllable frequency in light of the observation of phonological complexity.

In line with Wilshire \& Nespoulous (2003), we suggest that phonological complexity is playing a central role in phonological aphasia. Several studies have shown, for example, that the type of syllabic structure (CCV, CVC, CVCC, etc.) and the position of consonantal units (onset or coda position) play a considerable role in the phonological deficits in aphasia (Béland, 1985; Den Ouden, 2011; Den Ouden \& Bastiaanse, 2003; Prince, 2017; Romani \& Galluzi, 2005; Romani, Galuzzi, Bureca \& Olson, 2011; Wilshire \& Nespoulous, 2003).

This presentation will focus on defining the phonological complexity and its measurement based on evidence from empirical data in French people with stroke aphasia in its acute phase. The main goal is to explore the phonological difficulties depending on several variables - such as the type of the consonantal sequences and the position of the sequences. Specifically, attention will be focused on the role of complex syllabic structures in the use of repair strategies in aphasia.

\section{Methods}

Participants

Four monolingual native French-speakers with aphasia ( 4 men, mean age $=56$ years, from 48 to 66 years) were included in this experiment conducted in the stroke unit of Nantes' hospital 
(France). All the patients presented with acute aphasia characterized by a phonological deficit. All patients are monolingual, right-handed, and suffered from a stroke in the left hemisphere. Two of them were recorded day +6 and the two others were recorded day $+12 / 13$ after the stroke. Several types of acute aphasia cases are considered regarding their lesions and deficit, two of the patients present a similar conduction's aphasia while the two others present an anterior lesion associated to a Broca's aphasia type. Additional tests (sub-tests from the MT-86) were administered to participants in order to collect information about their cognitive and linguistic profile. At the same time, 20 healthy native French speakers were also tested.

\section{Procedure}

Participants with acute aphasia completed two tasks for this experiment: word repetition and reading tasks. The protocol is composed of (x32) bisyllabic and (x32) trisyllabic French words containing several consonantal sequences in initial and medial positions. 24 items contain initial binary consonantal sequences (tautosyllabic sequences such as obstruent-liquid and heterosyllabic sequences such as s+obstruent sequences), while 40 items contain binary medial consonantal sequences (tautosyllabic sequences such as obstruent-liquid and heterosyllabic sequences such as s+obstruent sequences, liquid-obstruent and obstruent-obstruent sequences). Words such as: moustache "mustache", stylo "pen", coccinelle "ladybug", citron "lemon", tortue, "turtle", were selected. The items were controlled, as far as possible, according to several variables that concern the present study: frequency, word length (bisyllabic vs trisyllabic words), clusters type (heterosyllabic vs tautosyllabic), cluster position (initial and medial positions), syllabic positions (onset and coda), semantic categories (substantives, fruits, daily objects, animals), and concreteness (Romani \& Olson, 2011). As suggested by Laganaro (2014), items were matched in the different tasks (repetition and reading) in order to observe if the nature of the task has an impact on the phonological transformations for each patient.

\section{Results}

Five types of repair strategies were noted: substitutions/shifting, deletions, epenthesis, metathesis and total reduction. They reveal an interaction between segments, syllables and positions. The preliminary results show that deletions and substitutions are the main strategies used by all PWA and that they affect specific contexts such as the syllabic position or the type of syllabic sequences, as in escargot, "snail": / ska o/ $\rightarrow$ [ka o], [ ka o] [ ka o], [ato], [esøka o], [teta o].

We suggest that phonological frequency is not the main variable in phonological aphasia and we will provide arguments for an effect of phonological complexity such as proposed by Wilshire and Nespoulous (2003). Individual analysis for each patient will be presented.

Exploring patterns of phonological transformation, we will show that regardless the type of aphasia, two principal types of transformations are observed in a phonological deficit during aphasia: (1.) Modification of the segmental structure, via substitutions, and (2.) Modification of the syllabic content, via deletions, shifting or epenthesis.

\section{Discussion}

Through this research, we also wish to contribute to the debate on the origin -phonetic and/or phonological -of the deficit and to highlight the role of syllable structures in this trouble.

\section{References}

Béland, R. (1985). Contraintes syllabiques sur les erreurs phonologiques dans l'aphasie. Thèse de doctorat. Canada: Université de Montréal.

Blumstein, S. (1978). Segment structure and the syllable in Aphasia, in Bell, A \& Hooper, JB. (eds.) Syllables and Segments. Amsterdam NHPC, 189-200. 
Buckingham, H. \& Buckingham, S. (2015). Phonological disorders. International Encyclopedia of the Social \& Behavioral Sciences. 18. Elsevier. 45-65.

Den Ouden, D.B. (2011). Phonological Disorders, in Botma, N.C, Kula, N. \& Nasukawa, K. (eds). Continuum Companion to Phonology. 320-340.

Den Ouden, D.B. \& Bastiaanse, R. (2005). Phonological encoding and conduction aphasia, in Hartsuiker, RJ. \& al. (eds). Phonological encoding and Monitoring in Normal and Pathological Speech. Psychology Press. 86-101.

Den Ouden, D.B. \& Bastiaanse, R. (2003). Syllable structure at different levels in the speech production process: Evidence from Aphasia, in Van de Weijer, J. \& Van de Heuven, V.J. \& Van der Hulst, H. (eds.) The Phonological Spectrum. II Suprasegmental Structure. Current issue in Linguistic Theory. 234, 81-107.

Goldrick, M. \& Rapp, B. (2007). Lexical and post-lexical phonological representations in spoken production. Cognition. 102, 219-260.

Laganaro, M. (2015). Évaluation des troubles phonologiques et phonétiques, in Séron, X. et M. Van der Linden (eds.). Traité de neuropsychologie clinique. Tome 1. 2e édition, 255-264. Marseille : Solal.

Laganaro, M. (2015). Paraphasies phonémiques et/ou phonétiques ? Des raisons et des difficultés de cette distinction. Revue de Neuropsychologie, 7 (1), 27-32.

Laganaro, M. \& Zimmermann, C. (2010). Origin of phoneme substitution and phoneme movement errors in aphasia. Language and Cognitive Processes, 25 (1), 1-37.

Levelt, W.JL, Roelofs, A. \& Meyer, A. (1999). A theory of lexical access in Speech production. Behavioral and Brain Sciences, 22, 1-75.

Nespoulous, JL., Baqué, L., Rosas, A., Marczyck, A. \& Estrada, M. (2013). Aphasia, phonological and phonetic voicing within the consonantal system: preservation of phonological oppositions and compensatory strategies. Language Sciences.

DOI: $10.1016 / j$.langsci.2013.02.015.

Prince, T. (2018). Déficit phonético-phonologique dans l'aphasie. Acte des XXXIIe Journées d'études sur la parole. JEP. Aix-en-provence, France.

Prince, T. (2017). Architectures des troubles phonético-phonémiques dans l'aphasie : des segments aux syllabes en français. Revue de Neuropsychologie, 81-98.

Romani, C., Galluzzi, C. \& Olson, A. (2011). Phonological-lexical activation: A lexical component or an output buffer? Evidence from aphasic errors. Cortex, 47, 217-235.

Romani, C., Galluzzi, C., Bureca, I. \& Olson, A. (2011). Effects of syllable structure in aphasic errors: implications for a new model of speech production. Cognitive Psychology, 151-192.

Romani, C., Galluzzi, C. (2005). Effects of syllabic complexity in predicting accuracy of repetition and direction of errors in patients with articulatory and phonological difficulties. Cognitive Neuropsychology, 22 (7), 817-850.

Wilshire, C.E., \& Nespoulous, J.L. (2003). Syllables as units in speech production: Data from aphasia. Brain \& Language. 84, 424-447. 


\title{
Neural tracking of semantics in natural speech
}

\author{
Jill Kries, Marlies Gillis, Jonas Vanthornhout, Tom Francart, Maaike Vandermosten \\ Experimental Oto-Rhino-Laryngology, Department of Neurosciences, KU Leuven, Belgium
}

\section{Introduction}

Cortical responses to artificial stimuli that target semantic processing have been widely explored with electroencephalography (EEG), resulting in late evoked potentials, i.e. N400, P300 (Hagoort \& Brown, 2000; Kutas \& Federmeier, 2011). Recently, ways to explore the brain's reactions to ecologically valid stimuli, such as a story, have been developed (Di Liberto \& Lalor, 2017; Lesenfants et al., 2019) and offer a high application potential for language disorders, such as aphasia or dyslexia (Di Liberto et al., 2018). Time-aligned vectors of semantic information are created and used for modelling an estimation of multi-channel cortical responses, which in turn are compared to the EEG signal measured on the human scalp (i.e. neural tracking), yielding a measure of semantic processing (Broderick et al., 2018). However, this semantic speech feature can be based on different types of information, i.e. word frequency, content words (Brodbeck et al., 2018) or dissimilarity vis-à-vis surrounding words (Broderick et al., 2018; Vanthornhout \& Francart, 2019). Furthermore, the parameters for data analysis can vary, e.g. size of word database or the number of surrounding words used for prediction (Broderick et al., 2018). Hence, depending on these types of information and parameters, different concepts of semantic processing are measured and therefore neural tracking analyses are prone to generate diverging results (Vanthornhout \& Francart, 2019).

In order to gain a better understanding of these impacting factors, we conducted two analyses for creating the semantic dissimilarity values of a 30-minute-long story. Namely, a comparison of one larger and one smaller word database was conducted as well as a comparison when taking into account all words or content words only. First, we hypothesized that the larger the word database used for estimation of semantic dissimilarity, the more dispersed the dissimilarity values would be. A larger database might assign more fine-tuned values to similar, yet different, semantic concepts and is thus more comparable to the semantic lexicon of the brain. Second, higher semantic dissimilarity values were hypothesized when taking into account content words alone instead of all words for the computation of semantic dissimilarity. Considering that words such as 'the' or 'here' are not adding up to the estimation of a certain word, it might be more beneficial to include only content words for computing the semantic dissimilarity feature. Furthermore, in order to see whether the type of stimulus story influences the brain's response to a certain speech model, i.e. neural tracking of semantic dissimilarity, a story modified for grammatically incorrect sentences was created, yielding higher values of semantic dissimilarity. We hypothesized increased levels of neural tracking for higher values of semantic dissimilarity, similar to the responses seen in EEG paradigms of late evoked potentials in response to semantically incongruent stimuli (Hagoort \& Brown, 2000).

\section{Methods}

\section{Semantic dissimilarity}

The semantic dissimilarity feature was computed by assigning a multi-dimensional vector to every word in the story (Broderick et al., 2018). This vector is based on the principle that words that often co-occur together have a similar value. Each word is correlated with the average of the 10 previous words and subsequently subtracted from 1 , resulting in a coefficient between 0 and 2 (Broderick et al., 2018).

In order to see the influence of the size of the word database on which the semantic dissimilarity feature of the story is based on, we compared the database word2vec, containing 500 000 words (Mikolov, Chen, Corrado, \& Dean, 2013), with the database fasttext, containing 2 
million words (Joulin et al., 2016). Thus, the outcome of this analysis consists of 2 semantic dissimilarity models of the same story, one for each word database. In order to explore the impact of taking into account content words alone in comparison to all words when computing the semantic dissimilarity model, a list of non-content words included in the story was extracted. Non-content words included adverbs, pronouns, determiners, prepositions, conjunctions and interjections. Other word categories, i.e. nouns, verbs and numerals, were classified as content words. Then, the number of non-content words was plotted for semantic dissimilarity models of both word databases.

\section{Neural tracking}

To explore the hypothesis that higher values of semantic dissimilarity might increase levels of neural tracking, a modified version of the original story 'De wilde zwanen' by H.C. Andersen was recorded. Two grammatical errors per sentence were introduced in $50 \%$ of sentences in randomized order (total number of errors $=308$ ). Seven different types of errors, randomized in order, were used, namely verb form, determiner, pronoun and reflexive, gender, preposition, negation and interrogative errors.

We recruited 15 participants, 2 participants with aphasia, 7 older adults and 6 younger adults, who listened to both versions of the story, grammatically correct (GC) and grammatically incorrect (GI), while we recorded EEG. The story was divided into parts of 5 minutes presented in alternating order. In order to monitor their level of attention, participants were asked to answer a question about the content of the story after each 5-minute part.

An encoding analysis was conducted with the semantic dissimilarity feature as input, using 15 minutes of the GC condition as training data and 15 minutes of each condition as test data. This analysis was repeated, however using the GI condition as training data. For validation of the data, the complete analysis was repeated using the speech envelope of the story as input, considering that this speech feature has consistently shown to be a good predictor for EEG responses to continuous acoustic stimuli (Di Liberto \& Lalor, 2017; Lesenfants et al., 2019).

\section{Results}

Both databases show semantic dissimilarity values ranging from 0.1 to 1.08 for the Dutch story 'De wilde zwanen'. However, the shape of the distribution changes. Whereas the values are negatively skewed for word2vec, the distribution is close to normal for fasttext. Interestingly, the distribution of non-content words is positively skewed for both databases in comparison to taking into account all words. This shows that content words have higher values of semantic dissimilarity and thereby confirms our hypothesis.

The neural tracking analysis shows significant correlations for all participants in both conditions, GC and GI. However, the number of significant EEG channels differs at group level and depending on which input feature was used, semantic dissimilarity or speech envelope. More detailed results will be presented at the conference.

\section{Discussion}

This investigation suggests taking into consideration the size of the word database, content words as well as the type of stimulus story when creating a model of semantic structure. By understanding how these factors influence the semantic model, more accurate and fine-tuned measures of semantic processing will emerge. Ultimately, using the semantic dissimilarity feature as input for predicting cortical responses to semantic cues in a continuous auditory stimulus can improve our understanding of the brain's semantic network.

\section{References}

Brodbeck, C., Presacco, A., \& Simon, J. Z. (2018). Neural source dynamics of brain responses 
to continuous stimuli : Speech processing from acoustics to comprehension. NeuroImage, 172(9), 162-174. https://doi.org/10.1016/j.neuroimage.2018.01.042

Broderick, M. P., Anderson, A. J., Liberto, G. M. Di, Crosse, M. J., \& Lalor, E. C. (2018). Electrophysiological Correlates of Semantic Dissimilarity Reflect the Comprehension of Natural, Narrative Speech. Current Biology, 28(5), 803-809. https://doi.org/10.1016/j.cub.2018.01.080

Di Liberto, G. M., \& Lalor, E. C. (2017). Indexing cortical entrainment to natural speech at the phonemic level : Methodological considerations for applied research. Hearing Research, 348, 70-77. https://doi.org/10.1016/j.heares.2017.02.015

Di Liberto, G. M., Peter, V., Kalashnikova, M., Goswami, U., Burnham, D., \& Lalor, E. C. (2018). Atypical cortical entrainment to speech in the right hemisphere underpins phonemic deficits in dyslexia. NeuroImage, 175(11), 70-79. https://doi.org/10.1016/j.neuroimage.2018.03.072

Hagoort, P., \& Brown, C. M. (2000). ERP effects of listening to speech: semantic ERP effects. Neuropsychologia, 38, 1518-1530.

Joulin, A., Grave, E., Bojanowski, P., Douze, M., Jégou, H., Mikolov, T. (2016). FastText.zip: Compressing text classification models. arXiv preprint, arXiv:1612.03651.

Kutas, M., \& Federmeier, K.D. (2011). Thirty years and counting: finding meaning in the N400 component of the event-related brain potential (ERP). Annual Review of Psychology, 62, 621-647.

Lesenfants, D., Vanthornhout, J., Verschueren, E., Decruy, L., \& Francart, T. (2019). Predicting individual speech intelligibility from the neural tracking of acoustic- and phonetic-level speech representations. Hearing Research, 380, 1-9. doi: 10.1016/j.heares.2019.05.006

Mikolov, T., Chen, K., Corrado, G., \& Dean, J. (2013). Efficient estimation of word representations in vector space. arXiv:1301.3781.

Vanthornhout, J., \& Francart, T. (2019). Semantic features in Dutch: a replication study of Brodbeck et al. and Broderick et al. Poster. 


\title{
Neuronavigated $1 \mathrm{~Hz}$ repetitive Transcranial Magnetic Stimulation (rTMS) in Chronic post-Stroke Aphasia
}

\author{
Anastasios M. Georgiou, Maria Kambanaros \\ Department of Rehabilitation Sciences, Cyprus University of Technology, Cyprus
}

\section{Introduction}

Aphasia, a significant aftermath of stroke, impacts more than a third of all survivors. Transcranial magnetic stimulation (TMS) is a novel approach aiming to influence language abilities by altering cortical excitability. The objective of this study was to investigate the effectiveness of $1 \mathrm{~Hz}$ rTMS as a standalone treatment for chronic aphasia post-stroke.

\section{Methods}

\section{Participants}

This was a single subject experimental design (SSED) trial, in which three adults who had suffered a single left hemispheric stroke at least six months before participating in the study, were treated with $1 \mathrm{~Hz}$ rTMS over the right pars Triangularis (pTr) for 10 consecutive days. Each participant underwent linguistic examination, with standardized and functional measures, and cognitive assessment two times before-, one day after- and two months post-treatment. Quality of life was also assessed once before treatment and two months post-treatment. Weighted statistics (WEST) were applied for the analysis of standardised language data, whereas functional communication followed thorough linguistic analysis.

\section{Results}

Behavioural changes included trends in improvement in verbal comprehension, naming and reading abilities in the short- and/or long-term. Regarding functional communication, the total number of narrative words showed a trend to increase post-treatment and two months post-treatment in one participant and; a trend to decrease in another participant. Quality of life (QoL) did not significantly change as a result of the treatment.

\section{Discussion}

Findings revealed that $1 \mathrm{~Hz}$ over the right pars triangularis (pTr), of the right Broca homologue shows promise to promote language gains. 


\title{
Linking production and comprehension -Investigating the lexical interface
}

\author{
Arushi Garg ${ }^{1}$, Vitória Piai ${ }^{1,2}$, Atsuko Takashima ${ }^{1,3}$, James M. McQueen ${ }^{1,3}$, Ardi Roelofs ${ }^{1}$ \\ ${ }^{1}$ Donders Institute for Brain, Cognition and Behaviour, Radboud University, Nijmegen, The \\ Netherlands \\ ${ }^{2}$ Radboudumc, Nijmegen, The Netherlands \\ ${ }^{3}$ Max Planck Institute for Psycholinguistics, Nijmegen, The Netherlands
}

\section{Introduction}

In a typical conversation, listening and speaking go hand in hand. Despite that, research into language production and comprehension has been conducted primarily independently of one another. This is evident in, and has probably resulted from, the distinction drawn between symptoms affecting individuals suffering from Broca's aphasia and Wernicke's aphasia and has been supported by the Wernicke-Lichtheim model and volumes of research that drew upon this work. However, recent clinical and non-clinical research suggests that the split between brain mechanisms underlying language production and comprehension is not clean and that several mechanisms are in fact shared between listening and speaking. People with aphasia presenting production difficulties have been shown to also have some difficulties in language comprehension and vice-versa. Recent studies investigating the core components underlying performance in individuals with aphasia showed that performance across speakers on different tasks is explained by factors such as lexical-semantic, phonological and executive-cognitive deficits rather than by a strict distinction between production and comprehension (Butler, Lambon Ralph, \& Woollams, 2014; Fridriksson et al., 2016)

It is thus evident that there is a need to study the mechanisms shared between language production and comprehension. In single-word studies, it is believed that the lexical and conceptual levels are shared between listening and speaking. According to a leading theory (Levelt, Roelofs, \& Meyer, 1999), lemma representations help speakers map the meaning of words to their sounds and listeners map the sounds to their meanings. Additionally, evidence from a variety of studies (including meta-analyses and studies with healthy subjects as well as individuals with language disorders) suggests that lemmas are likely localised in the middle portion of the left middle temporal gyrus (left mMTG) (Dronkers, Wilkins, Van Valin, Redfern, \& Jaeger, 2004; P. Indefrey \& Levelt, 2004; Peter Indefrey, 2011; Piai, Roelofs, Jensen, Schoffelen, \& Bonnefond, 2014; Schwartz et al., 2009) . Roelofs (2014) applied the lemma theory to aphasia using a model compatible with the suggestion that lemma representations are localised in the left mMTG, simulating both production and comprehension deficits. Nonetheless, the claims regarding the shared nature of lemmas between production and comprehension and its localisation in the brain have not been empirically established.

These questions have been challenging due to the abstract nature of lemma representations. The sole purpose of a lemma is to link together various types of representations associated with a word (conceptual, syntactic and phonological representations). Thus, any kind of spoken single-word task would always involve one or more of these three types of representations in addition to lemma representations, and therefore present a potential confound. To work around this confound, we attempted to answer the questions above using an fMRI study in which subjects perform multiple tasks. The tasks are either production or comprehension tasks and they are targeted at the different levels of representation -conceptual or syntactic -that the lemma of a word connects. By analysing the activity that is common to all four tasks, we hope to find out (1) whether there is a brain region that is activated in both production and 
comprehension tasks, (2) whether this same region is activated in the task regardless of the level of the representation -conceptual or syntactic - that the task targets and (3) whether such a region is located in the left middle MTG.

\section{Methods}

\section{Subjects and Design}

This experiment uses behavioural and fMRI data from 32 healthy native speakers of Dutch who perform four single-word tasks in Dutch. Each participant performed two production and two comprehension tasks. For production, participants named pictures without determiner (bare picture naming) and with determiner (gender-marked picture naming). For comprehension tasks, participants judged whether the spoken words denoted something man-made or natural (semantic classification -conceptual level) and whether the words were associated with the Dutch determiners "de" or "het" (gender classification -syntactic level).

\section{fMRI data acquisition and analysis}

Participants are scanned with a Siemens 3T scanner with a multi-band multi-echo EPI sequence (TR-1.5 seconds).

Contrast images of each task against the implicit baseline for each participant will be compared across participants. Since we expect the lemma representation to reside in the left middle temporal cortex, we will further look into the brain responses within this area across the four tasks. We expect to find common activation across all four tasks in this area.

\section{Results}

We are still in the process of acquiring the data from the last three participants. The outcome of the latest analyses of the fMRI data will be presented at the meeting.

\section{Discussion}

If we are able to localise lemma representations, we can show that there are shared mechanisms between production and comprehension and that the lexical level, in particular, is shared. We will be able to establish that the left temporal lobe plays a major role concerning the lexical aspects of language processing, explaining why damage to the region will have major implications for the language capabilities of individuals. More importantly, such a finding can be useful in helping establish prognosis for people suffering from such a damage.

\section{References}

Butler, R. A., Lambon Ralph, M. A., \& Woollams, A. M. (2014). Capturing multidimensionality in stroke aphasia: mapping principal behavioural components to neural structures. Brain, 137(12), 3248-3266.

Dronkers, N. F., Wilkins, D. P., Van Valin, R. D., Redfern, B. B., \& Jaeger, J. J. (2004). Lesion analysis of the brain areas involved in language comprehension. Cognition, 92(12), 145-177.

Fridriksson, J., Yourganov, G., Bonilha, L., Basilakos, A., Ouden, D.-B. D., \& Rorden, C. (2016). Revealing the dual streams of speech processing. Proceedings of the National Academy of Sciences, 113(52), 15108-15113.

Indefrey, P., \& Levelt, W. J. M. (2004). The spatial and temporal signatures of word production components. Cognition, 92(1-2), 101-144.

Indefrey, Peter. (2011). The Spatial and Temporal Signatures of Word Production Components: A Critical Update. Frontiers in Psychology, 2(October), 1-16.

Levelt, W. J. M., Roelofs, A., \& Meyer, A. S. (1999). A theory of lexical access in speech 
production. BEHAVIORAL AND BRAIN SCIENCES, 22, 1-75.

Piai, V., Roelofs, A., Jensen, O., Schoffelen, J. M., \& Bonnefond, M. (2014). Distinct patterns of brain activity characterise lexical activation and competition in spoken word production. PLoS ONE, 9(2).

Roelofs, A. (2014). A dorsal-pathway account of aphasic language production: The WEAVER ++ / ARC model. Cortex, 59(1874), 33-48.

Schwartz, M. F., Kimberg, D. Y., Walker, G. M., Faseyitan, O., Brecher, A., Dell, G. S., \& Coslett, H. B. (2009). Anterior temporal involvement in semantic word retrieval: voxelbased lesion-symptom mapping evidence from aphasia. Brain, 132(12), 3411-3427. 


\title{
The Effect of tDCS on Syntactic Processing in Aphasia
}

\author{
Dilek Eroğlu Uzun ${ }^{1}$, Serkan Şener ${ }^{2}$, Barış Metin ${ }^{1}$ \\ ${ }^{1}$ Uskudar University \\ ${ }^{2}$ Yeditepe University, Istanbul, Turkey
}

\begin{abstract}
Introduction
Aphasic individuals syntactic processing abilities can be destroyed, especially their comprehension of complex syntactic structures. Neuromodulation techniques such as tDCS (transcranial direct current stimulation) can enhance their comprehension abilities of syntactic structures by directing their brain activation. There are some studies that attempts to find if there is any recovery effect of tDCS on aphasia (1). Their main hypothesis is increasing stimulation in ipsilesilesional area or suppressing stimulation in contralesional area in the brain can enhance language abilities (2-3).
\end{abstract}

The aim of the current study was to evaluate the effect of tDCS on syntactic processing by activating or suprressing Wernicke's area in different conditions (such as anodal, cathodal)

\section{Methods}

Participants

8 individuals with aphasia participated in our study. The patients were recruited at Uskudar University Speech Therapy Research and Application Center where they were admitted to tDCS sessions. All participants or their caregivers signed a written informed consent before starting to our sessions. Demographic information of aphasic individuals are given in table 1 below.

Our inclusion criteria were: (a) native speaker of Turkish; (b) age between 40-80 years; (c) left hemisphere stroke; (d) minimum 6 months post-onset; (e) mild to moderate aphasia; (f) absence of seizures; (g) absence of other psychiatric or neurologic disorders; (h) absence of intracranial metal implants; (i) right handedness (j) mild to moderate auditory comprehension.

3 age matched individuals who have no neurological or psychiatric disorders included in the study as control group, just for syntactic task application. The type and degree of aphasia were assessed using The Test of Language Assessment in Aphasia (ADD) (4).

Regarding our inclusion criteria for the present study, it was important that our patients were in the chronic stage because we wanted to eliminate potential spontaneous recovery effects. We also did not include severe aphasic individuals that had severe difficulties in processing syntactic structures since the present study aimed at investigating syntactically complex constructions.

\section{Transcranial Direct Current Stimulation}

We applied 2mA anodal, cathodal current to different groups for $20 \mathrm{~min}$. over a period of one week (consecutive 5 sessions). The active electrode (anodal) was placed on Wernicke's area on left hemisphere (3 aphasic patients), the reference electrode (cathodal) was placed on the right hemisphere homolog for Wernicke's area (3 aphasic patients) and we also have a control group (3 normal individuals) that we did not use tDCS.

\section{Transcranial Direct Current Stimulation}

In this experiment in order to evaluate aphasic individuals syntactic processing abilities we have used black-white drawings of different syntactic structures, these syntactic structures consist of 
four linguistic structures that are different in complexity; (1) active (ACT), (2) passive (PASS), (3) object relative (OR), (4) subject relative (SR). Every sentence that belong to one syntactic structure have one target drawing and one distractor that is syntactically similar to the target sentence. Every target structure have 20 sentences, we have 80 target sentence in total. All these drawings that belong to different structures were given to patients via computer program that meausure reaction time (RT) and true-false (T/F) scores. Computer screen was devided into four sections for different pictures (one target, one syntactic distractor, two irrelevant sentence pictures.

\section{Procedure}

We tested all aphasic indiviuals with The Test of Language Assessment in Aphasia. In the beginning of every session we applied our linguistic task that consist of 20 target structure which consist of four different syntactic structure in compelxity. Five different lists were prepaired for every session to avoid learning effect and in the first session aphasic individuals had a trial of the task with four sentences that is not belong to the first list. For every list there were five sentences from each structures, these five sentences were randomly selected. After linguistic task, we applied 2mA tDCS for $20 \mathrm{~min}$. In anodal condition we applied tDCS on left Wernicke's area (CP5), in cathodal stimulation we applied tDCS on right homologue area for Wernicke (CP6). Immediately after tDCS session we applied our linguistic task again. Five consecutive sessions were conducted in one week of period.

\section{Statistical Analysis}

This is a mixed-model design experimental study in which we compared conditions (anodal, cathodal), duration (RT), and accuracy before and after tDCS sessions in between and within groups. Statistical analyses were performed in SPSS IBM 20.0 version using paired samples t-test and independent samples t-test. In order to test difference between anodal and cathodal stimulation groups we used independent samples t-test, but for testing pretest-posttest differences in one group (anodal or cathodal) we used paires samples t-test.

\section{Results and Discussion}

Our results were as follows, anodal stimulation after tDCS sessions showed that there was a significant difference between the first and the last sessions of aphasic individuals ; the RT comparison of four syntactic structures resulted in a significant difference just in OR type sentences in anodal stimulation; the RTcores of ORs in anodal stimulation increased significantly compared to ACT, PASS, and SR; comparison of T/F scores of four syntactic structures demonstrate that there was no statistically significant difference between ACT, OR, and PASS sentences between 1st and 5th sessions after tDCS but there was a significant difference for SR sentence type; the results of cathodal stimulation after first and last tDCS stimulation demonstrate that there was no significant difference between $\mathrm{T} / \mathrm{F}, \mathrm{RT}$, and distractor results and also there was no significant difference between four syntactic structure processing, there was a decrease in $\mathrm{T} / \mathrm{F}$ scores of PASS in cathodal stimulation. In $\mathrm{T} / \mathrm{F}$ comparison between groups results showed that anodal groups mean $(5,67)$ was greater than cathodal groups mean $(1,33)$, we can interpret this result as A-tDCS is more effective on syntactic processing compared to C-tDCS.

Our results revealed that A-tDCS can improve language skills by decreasing bilateral activation (5-6) but C-tDCS had negative effect on syntactic processing as in the study of Liuzzi et. al., (2010) that they study action and object learning. 


\section{References}

1 Schlaug G, Marchina S, Wan CY. (2011). The use of non-invasive brain stimulation techniques to facilitate recovery from post-stroke aphasia. Neuropsychology Review, 21:288301.

2 Crinion JT, Leff AP. (2007). Recovery and treatment of aphasia after stroke: functional imaging studies. Current Opinion in Neurology, 20:667-73.

3 Fridriksson J, Bonilha L, Baker JM, Moser D, Rorden C. (2010). Activity in preserved left hemisphere regions predicts anomia severity in aphasia. Cerebral Cortex, 20:1013-9.

4 Maviş, İ., \& Toğram, B. (2009). Language assessment test for aphasia (ADD). Ankara: Detay publishing

5 Meinzer, M., Lindenberg, R., Antonenko, D., Flaisch, T., \& Floel, A. (2013). Anodal transcranial direct current stimulation temporarily reverses age-associated cognitive decline and functional brain activity changes. The Journal of Neuroscience, 33(30), 12470e12478. http://dx.doi.org/10.1523/ JNEUROSCI.5743-12.2013.

6 Holland, R., Leff, A. P., Josephs, O., Galea, J. M., Desikan, M., Price, C. J., et al. (2011). Speech facilitation by left inferior frontal cortex stimulation. Current Biology, 21(16), 1403e1407. http://dx.doi.org/10.1016/j.cub.2011.07.021. 


\title{
Linguistic parameters in an app-based assessment of German verbs and nouns in aphasia
}

\author{
Dörte de Kok ${ }^{1}$, Sarah Hanekamp ${ }^{2}$, Roelien Bastiaanse ${ }^{1,3}$ \\ ${ }^{1}$ Center for Language and Cognition, University of Groningen, The Netherlands \\ ${ }^{2}$ Master in Linguistics, University of Groningen, The Netherlands \\ ${ }^{3}$ Center for Language and Brain, Higher School of Economics, Moscow, Russia
}

\section{Introduction}

People with aphasia often experience (specific) problems with the production and comprehension of verbs (Bastiaanse \& Bol, 2001; Jonkers \& Bastiaanse, 2006). Several linguistic parameters have been argued to influence their performance, such as age of acquisition (Nickels \& Howard, 1995), transitivity (Jonkers \& Bastiaanse, 1996), imageability (e.g., Franklin et al. 1995) and instrumentality (Jonkers \& Bastiaanse, 1996, 2006). While there also seems to be an effect of frequency on action naming tasks, this is highly debated and did not hold, when tangled apart from age of acquisition effects (e.g., Nickels \& Howard, 1995, Bastiaanse et al., 2015).

In order to provide a model-based assessment of the specific problems of people with aphasia, the Dutch version of the Verb and Sentence Test (VAST) was developed (Bastiaanse et al., 2000). The analysis and interpretation of this test, however, are rather complicated and require a thorough understanding of the underlying linguistic theory. A new, app-based version of the test has been developed for Dutch, making administration, scoring and interpretation more straight-forward (cf. De Kok et al., 2013). Bastiaanse and colleagues (2015) have shown that the performance on action and object naming was influenced by age of acquisition and imageability. The object naming performance was furthermore also dependent on word frequency.

In the meanwhile, a German version of the test has been developed, also administered appbased. The test-construction has been completed and data from non-brain-damaged participants and people with aphasia are being collected. The goal of the current research is therefore twofold: the gathered data will serve to create the normative data for the diagnostic tool and will also be analyzed with regard to the effect of the controlled linguistic parameters on the performance of the people with aphasia.

\section{Methods}

\section{Test construction}

The test consists of six subtests, "verb comprehension", "action naming", "filling in infinitives in sentences", "filling in finite verbs in sentence", "object naming", and "semantic association of verbs". These tests were carefully constructed taking into account various relevant linguistic variables, such as lemma frequency, age of acquisition, imageability, length in phonemes and visual complexity of the images (black and white line drawings). Additionally, the parameters instrumentality and transitivity were controlled for the verb tasks. For variables that could not be looked up in existing corpora, ratings were obtained. This is for example the case for "visual complexity" and "imageability".

The receptive tasks are scored automatically (based on the chosen image), taking accuracy and error type into consideration. For the productive tasks, audio-recordings are obtained that can then be scored within the app. After scoring, the app will provide an automatically generated result overview. The result overview for both receptive and productive tasks includes an analysis of the influencing linguistic parameters. For the current study, however, results were analyzed on an item-basis outside the app. 


\section{Participants}

Both a group of non-brain-damaged control participants and a group of people with aphasia are participating in the study. The non-brain-damaged participants will be matched in age and education level to the group of people with aphasia. In total we aim for 100 participants without brain damage and 50 participants with aphasia, distributed over different syndromes and severities. All participants will have German as a native language. So far, four participants with aphasia have concluded testing. All four participants are male, in the chronic phase and show a moderate form of aphasia with word-finding difficulties. Three of the participants exhibit non-fluent type aphasia (P2, P3 and P4) and one fluent aphasia (P1).

\section{Results}

The effects of linguistic parameters were analyzed on an individual level for the four participants. Effects for parameters with interval data (frequency, age-of-acquisition, imageability, length in phonemes) were evaluated with one-tailed Mann-Whitney-U tests and effects for parameters with categorical data (transitivity, instrumentality) with one-tailed Fisher's exact tests.

Table 1. Overview of found effects for the four participants with aphasia

\begin{tabular}{|c|c|c|c|c|c|c|c|c|c|}
\hline & & $\begin{array}{l}\% \\
\text { cor. }\end{array}$ & freq. & AoA & $\begin{array}{l}\text { Image- } \\
\text { ability }\end{array}$ & $\begin{array}{l}\text { Length } \\
\text { (phon.) }\end{array}$ & $\begin{array}{l}\text { vis. } \\
\text { compl. }\end{array}$ & Transitivity & Instrumentality \\
\hline \multirow{4}{*}{ 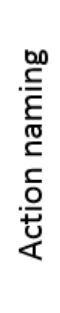 } & P1 & $69 \%$ & $\begin{array}{l}\mathrm{U}=139 \\
\mathrm{p}=0.002\end{array}$ & $\begin{array}{l}U=110.5, \\
p=0.000\end{array}$ & n.s. & n.s. & $\begin{array}{l}U=136.5, \\
p=0.032\end{array}$ & $p=0.016$ & n.s. \\
\hline & P2 & $83 \%$ & n.s. & n.s. & n.s. & n.s. & n.s. & n.s. & $p=0.042$ \\
\hline & P3 & $77 \%$ & n.s. & n.s. & n.s. & n.s. & n.s. & n.s. & $\begin{array}{l}\text { n.s. } \\
(p=0.067)\end{array}$ \\
\hline & P4 & $50 \%$ & n.s. & $\begin{array}{l}U=222.5, \\
p=0.041\end{array}$ & n.s. & $\begin{array}{l}U=186, \\
p=0.004\end{array}$ & n.s. & n.s. & n.s. \\
\hline \multirow{4}{*}{ 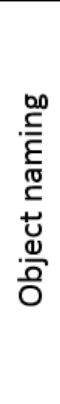 } & P1 & $84 \%$ & n.s. & n.s. & n.s. & n.s. & $\begin{array}{l}U=86.5, \\
p=0.015\end{array}$ & - & - \\
\hline & P2 & $88 \%$ & $\begin{array}{l}U=68.5 \\
p=0.033\end{array}$ & n.s. & n.s. & n.s. & $\begin{array}{l}U=66, \\
p=0.027\end{array}$ & - & - \\
\hline & P3 & $92 \%$ & $\begin{array}{l}U=24, \\
p=0.006\end{array}$ & $\begin{array}{l}U=16, \\
p=0.02\end{array}$ & n.s. & $\begin{array}{l}U=41.5, \\
p=0.041\end{array}$ & n.s. & - & - \\
\hline & P4 & $48 \%$ & $\begin{array}{l}\text { n.s. } \\
(U=228.8, \\
p=0.053)\end{array}$ & n.s. & $\begin{array}{l}U=195 \\
p=0.016\end{array}$ & $\begin{array}{l}U=154.5, \\
p=0.001\end{array}$ & $\begin{array}{l}U=225, \\
p=0.046\end{array}$ & - & - \\
\hline
\end{tabular}

The table above shows the results on action and object naming for all four participants. Reported effects are in the following direction: transitive items were more often correct than intransitive items and instrumental items were more often correct than non-instrumental items. For the other parameters the following holds (if an effect is reported): the correct items are of higher frequency, lower age of acquisition, higher imageability, lower length and lower visual complexity than the incorrect items.

On the verb comprehension task, three of the participants with aphasia scored at ceiling (maximally one error). Only P1 scored worse, with $85 \%$ correct responses. He did not show significant effects for any parameter, but a tendency to comprehend non-instrumental verbs better than instrumental ones $(p=0.06$, Fisher's exact test). 


\section{Discussion}

The presented results only provide a preview of the expected data. Once more participants are included, analyses on group level will be carried out. These will include comparisons between tasks, such as object vs. action naming and infinitive vs. finite verb production. Also, a larger group will allow to perform a (mixed model) regression analysis, taking all factors into account at the same time and disentangle them. Due to the close relationship between frequency and age of acquisition the analysis presented above may be confounded. These extended analyses will be presented at the conference.

Although this is just a preliminary analysis, some conclusions can be drawn. All controlled and reported parameters are relevant to the diagnostic tool. Each parameter influences the performance of at least one participant (out of four). Furthermore, we can also conclude that the developed test is sensitive enough to test a participant's performance on the subtest and to detect the influence of linguistic parameters on an individual level. This is a necessary prerequisite for a diagnostic tool that aims to evaluate the underlying deficit of a participant with aphasia.

\section{References}

Bastiaanse, R. \& Bol, G. (2001). Verb Inflection and Verb Diversity in Three Populations: Agrammatic Speakers, Normally Developing Children, and Children with Specific Language Impairment (SLI). Brain and Language, 7r, 274-282.

Bastiaanse, R., Maas, E. \& Rispens, J. (2000). Werkwoorden- en Zinnentest (WEZT). Lisse, Swets Test Publishers.

Bastiaanse, Y., Wieling, M. \& Wolthuis, N. (2015). The role of frequency in the retrieval of nouns and verbs in aphasia. Aphasiology, 30, 1221-1239.

de Kok, D., Wolthuis, N., \& Bastiaanse, R. (2013). VAST-App - testing verbs and sentences with the iPad. Stem-, Spraak- en Taalpathologie 18 (S01), 86-89.

Franklin, S., Howard, D. and Patterson, K. (1995). Abstract word anomia. Cognitive Neuropsychology, 12, 549-566.

Jonkers, R., \& Bastiaanse, R. (1996). The influence of instrumentality and transitivity on action naming in Broca's and anomic aphasia. Brain and Language, 55, 37-39.

Jonkers, R. \& Bastiaanse, R. (2006). The influence in instrumentality and name-relation to a noun on verb comprehension in Dutch aphasic speakers. Aphasiology, 20, 3-16.

Nickels, L., \& Howard, D. (1995). Aphasic naming: What matters? Neuropsychologia, 33, 1281-1303. 


\title{
Understanding language recovery in stroke patients by including neuroanatomical and behavioural measures of learning potential
}

\author{
Maaike Vandermosten ${ }^{1}$, Klara Schevenels ${ }^{1}$, Inge Zink ${ }^{1}$, Bert De Smedt ${ }^{2}$ \\ ${ }^{1}$ Department of Neuroscience, KU Leuven, Belgium \\ ${ }^{2}$ Department of Psychology and Educational Sciences, KU Leuven Belgium
}

\section{Introduction}

In the first months post-stroke, there is often some spontaneous and intervention-induced recovery, but for most patients a chronic deficit remains1. Identifying the early predictors of aphasia recovery cannot only inform the patient and their family about what to expect, but also has the potential to lead to better choices in intervention and to maximize recovery. However, to date, individual language recovery in patients with aphasia is hard to predict. In literature, the combination of demographic factors (such as gender, age or handedness) and language-related behavioural predictors (initial severity and type of language problems) can at best explain one third of the variance in aphasia outcome2. Neuroimaging studies have tried to improve this prediction by adding lesion-related neurological predictors, such as lesion size and location3,4. Although this has lead to an increase in explained variance up to 50\%5, there remains a large proportion of unpredicted variance. The aim of this project is to improve the prognosis of language by shifting the traditional focus from the brain damage and the behavioural deficits to intact brain structures and cognitive abilities that can assist in (re)learning language. Given the importance of statistical learning in the acquisition of the mother tongue in infants6, as well as in the acquisition of a second language in adults7,8, it shows great potential to explain the variability in relearning a language in persons with aphasia. At the neural level, the hippocampus is well-known for its involvement in statistical learning9-11, though this has not yet been tested with regard to language recovery in persons with aphasia. Hence, the first objective is to demonstrate that information on dynamic language learning potential (statistical learning) improves the prediction of language recovery, above the traditional measures of language skills, lesion information and demographic factors (e.g. age, gender, multilingualism). Our second objective is to find a neuroanatomical correlate of statistical learning outcomes, hypothesized to be located in the hippocampus, and to examine whether it can further improve aphasia prognosis. The third objective is to monitor the neuroplasticity during language recovery. Bilateral neural changes related to language recovery occur over time12. However, information on the role of subcortical structures and white matter pathways herein, such as the hippocampus, its connections to cortical language regions and the arcuate fasciculus, is very scarce.

\section{Methods}

Experimental design and participants

First, we recruited 25 younger (20-40 years old) and 25 older healthy adults $(\mathrm{N}=40,55-75$ years old) to assess statistical learning abilities in preparation of the behavioural data collection in aphasic patients. Therefore, we explored different statistical learning tasks that differed in the modality that was targeted (auditory versus visual) and the type of statistical learning (conditional versus distributional versus procedural). Based on the results which indicated the statistical learning tasks that were above chance level in older adults, we selected a visual and auditory conditional learning task and a procedural learning task to assess the learning potential in persons with aphasia. Second, based on screening more than 200 stroke patients in the first week post-stroke, we included 20 patients with aphasia of whom we collected behavioural language data (phonology, semantics, syntax and daily communication skills) and MRI-data (diffusion MRI, T1 and resting state fMRI) in the acute phase (one week post-stroke) as well as in the sub-acute phase (3 months post-stroke). In addition, statistical learning experiments 
were assessed in the sub-acute phase.

\section{Analyses}

The T1-weighted image not only enabled us to investigate the size and site of the lesion, but also to conduct detailed morphological analyses of the undamaged hippocampus. We first performed these analyses by manual delineation of the structures, using the EADC-ADNI Harmonized Hippocampal Protocol (HarP)13 for the hippocampus, and in the next months we will complement this by more fine-grained Freesurfer analyses allowing to examine different segments of the hippocampus14. In addition to T1-weighted images, we collected diffusion weighted images to investigate (the changes in) the white matter pathway highly involved in language, namely the arcuate fasciculus, and the white matter pathways connecting the hippocampus with the language cortex. The arcuate fasciculus was manually delineated in native space15 and for the hippocampal fibers, the segmented hippocampus was taken as a seed.

Once data collection of all these longitudinal behavioural and anatomical measures is finished, we will describe and predict the behavioural and neural changes in the first 3 months of aphasia recovery in different language domains over time, using growth curve modelling. Our final independent variables will include demographic variables, the subjects' statistical learning potential, the initial language measurements (initial aphasia severity and type), lesion size, lesion site and (changes in) the detailed morphological measures of the hippocampus, the arcuate fasciculus and the white matter pathways connecting the hippocampus with perisylvian language areas.

\section{Results}

At the moment, we have analyzed the behavioral language data and the diffusion MRI-data of 20 patients with aphasia in the acute phase. These results indicated a significant positive correlation between the fractional anisotropy in the left posterior arcuate fasciculus and language performance in the acute phase $(\mathrm{r}=0.54, p<.05)$. In a next step, we will examine whether these results are explained by the size and location of the lesion and how this predicts language outcomes. The next coming months, we plan to analyze the data of the sub-acute phase, namely the language, statistical learning and MRI data. This will allow us to investigate the interplay between these variables and their role in language recovery and neuroplasticity.

\section{Discussion}

The investigation of language recovery predictors has received some attention in aphasia literature, although these studies do not take into account compensatory neurocognitive factors. Increasing prediction accuracy by including statistical learning and neuroanatomical measures of undamaged brain areas as well as information on white matter pathways, will shift the attention of the scientific community in research domains that undertake neuroprediction to the potential of compensatory mechanisms.

\section{References}

1 Maas, M. B. et al. The Prognosis for Aphasia in Stroke. J. Stroke Cerebrovasc. Dis. 21, 350-357 (2012).

2 van Bragt, P. J. et al. Predicting outcome in a postacute stroke rehabilitation programme. Int. J. Rehabil. Res. 37, 110-117 (2014).

3 Goldenberg, G. \& Spatt, J. Influence of Size and Site of Cerebral Lesions on Spontaneous Recovery of Aphasia and on Success of Language Therapy. Brain Lang. 47, 684-698 (1994).

4 Naeser, M. A. \& Palumbo, C. L. Neuroimaging and Language Recovery in Stroke. Journal of Clinical Neurophysiology 11, 150-174 (1994). 
5 Hope, T. M. H., Seghier, M. L., Leff, A. P. \& Price, C. J. Predicting outcome and recovery after stroke with lesions extracted from MRI images. YNICL 2, 424-433 (2013).

6 Kuhl, P. K. Early language acquisition: cracking the speech code. Neuroscience 5, 831-843 (2004).

7 Hamrick, P. A Role for Chunk Formation in Statistical Learning of Second Language Syntax. Lang. Learn. 64, 247-278 (2014).

8 Frost, R., Siegelman, N., Narkiss, A. \& Afek, L. What predicts successful literacy acquisition in a second language? Psychol. Sci. 24, 1243-1252 (2013).

9 Squire, L. R., Stark, C. E. L. \& Clark, R. E. The medial temporal lobe. Annu. Rev. Neurosience 27, 279-306 (2004).

10 Schapiro, A. C., Gregory, E., Landau, B., McCloskey, M. \& Turk-browne, N. B. The Necessity of the Medial Temporal Lobe for Statistical Learning. J. Cogn. Neurosci. 26, 1736-1747 (2014).

11 Schlichting, M. L., Guarino, K. F., Schapiro, A. C., Turk-browne, N. B. \& Preston, A. R. Hippocampal Structure Predicts Statistical Learning and Associative Inference Abilities during Development. J. Cogn. Neurosci. 29, 37-51 (2016).

12 Schevenels, K., Price, C., Zink, I., De Smedt, B., Vandermosten, M. (in preparation). A review on treatment-induced neuronal reorganization in aphasia.

13 Boccardi, M. et al. Delphi definition of the EADC-ADNI Harmonized Protocol for hippocampal segmentation on magnetic resonance. Alzheimer's Dement. 11, 126-138 (2015).

14 Iglesias, J.E., Augustinack, J. C., Nguyen, K., Player, C. M., Player, A., Wright, M., ... Van Leemput, K. (2015). A computational atlas of the hippocampal formation using ex vivo, ultra-high resolution MRI: Application to adaptive segmentation of in vivo MRI. NeuroImage 115, 117-137.

15 Vandermosten, M., Boets, B., Poelmans, H., Sunaert, S., Wouters, J., Ghesquière, P. (2012). A tractography study in dyslexia: Neuroanatomical correlates of orthographic, phonological and speech processing. Brain 135, 935-948. 


\title{
Assessing verb selection using MEG. A precise methodology for language presurgical mapping
}

\author{
Elena Salillas ${ }^{1}$, Domenico D'Avella ${ }^{1}$, Giorgio Arcara ${ }^{2}$, Francesco Piccione ${ }^{2}$, Sara Zago ${ }^{2}$, Silvia \\ di Tomasso ${ }^{2}$ and Carlo Semenza ${ }^{1,2}$ \\ ${ }^{1}$ Department of Neurosciences, University of Padova \\ ${ }^{2}$ I.R.C.C.S. Fondazione Ospedale San Camillo
}

\begin{abstract}
Introduction
Identifying the individual brain location of eloquent areas at the single case level, as well as the determination of language lateralization, is crucial before surgery. Accuracy in identifying language areas presurgically is currently not optimal, however, in comparison to sensorimotor functions (De Witte and Mariën, 2013). Here we present a new paradigm, using magnetoencephalography (MEG), that may be usefully applied to determine language lateralization and localization of language functions in presurgical patients.
\end{abstract}

Among other tasks, many protocols involve the use of verb generation (e.g. Bizzi et al., 2008; Roux et al., 2003; Papagno et al., 2011), often contrasted with suboptimal baselines (or to zero), which reduces precision in the mapping of specific language areas. Here we propose an adaptation of the verb generation task, according to the procedures introduced by ThompsonSchill et al $(1997,1998)$, in the context of presurgical mapping. These authors showed the repeated involvement of the left inferior frontal gyrus, including Broca's area, depending on the degree of semantic competition imposed by the verbs. Such a modified verb generation task might involve a precise initial cue for determining language lateralization, including the detection of a possible recruitment of homologue language areas due to reorganization in some clinical populations (Rosenberg et al., 2009).

\section{Methods}

Participant

A left handed 15 y.o. female patient, with early onset intractable left temporal epilepsy was studied with MEG. In this case localization of language becomes relevant also due to handedness. A group of 12 healthy Italian subjects participated in the building of stimuli.

\section{Stimuli}

A pool of 66 nouns were classified as imposing high or low selection demands. As in Thompson Schill, et al. (1998), healthy participants were asked to generate a verb from each noun. A ratio of the relative response frequency of the most common completion to the relative response frequency of the second most common completion was calculated as a measure of response strength. We furthermore considered the number of different nouns given in the index. This response strength was used to rate the items according to the imposed selection effort: the larger the ratio between the most frequently associated verb and the second most associated verb, the lower the selection imposed by the noun. Hence larger ratios were related to lower selection and smaller ratios to higher selection.

\section{Procedure and analyses}

During MEG registration, the patient was presented with the 66 auditory nouns, to which she had to generate a related verb. After listening to the word, a visual cue requested the emission of the verb. Event related magnetic fields (ERFs) were measured time-locked to the visual cue, segmented and averaged according to the semantic selection condition, in two conditions, low / high selection. Cortex sources related to the ERFs were estimated using Minimum Norm 
Estimates for each item.

\section{Results}

The averaged Event Related Fields (ERFs) showed a negative slow component ( 260-860 ms. ), upon the presentation of the visual cue for verb production, with larger amplitude depending on the degree of selection demands imposed by the noun condition (High selection $(\mathrm{n}=22)>$ Middle selection $(n=22)>$ Low selection $(n=22))$. Crucially, the estimated sources of this effect showed a maxima in right BA 45 (pars triangularis). Minor activations were also found in the left hemisphere, in the left BA 45 and middle frontal gyrus, and in the middle anterior right temporal gyrus. Ratio effect was maximal in the 460 to $660 \mathrm{~ms}$. and located in right BA 45. One-sample t-test analyses by items, considering the obtained ratio as a covariate, showed that the activity of this area was negatively related to the ratio for each item. That is, the intensity of activation in this area was linearly gradated according to the semantic selection condition, with higher activation as the selection became higher (i.e. lower ratios).
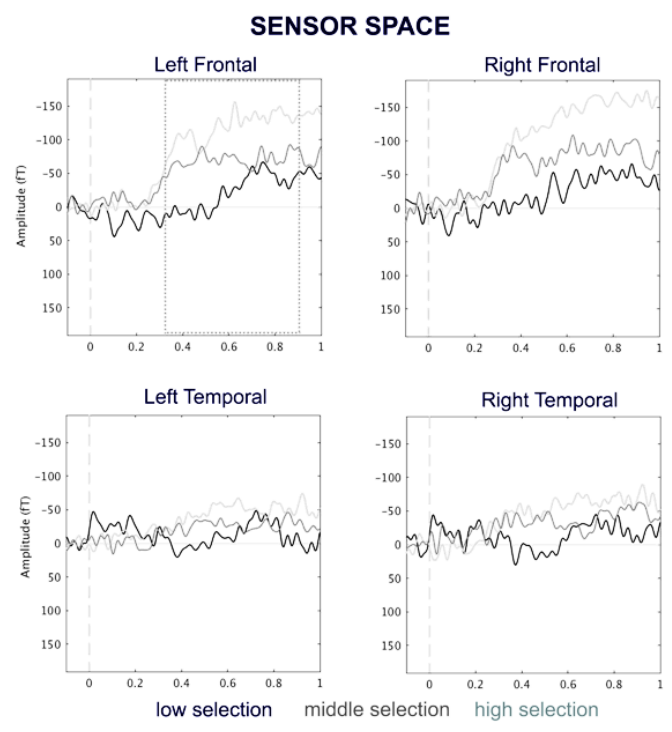

SOURCE SPACE
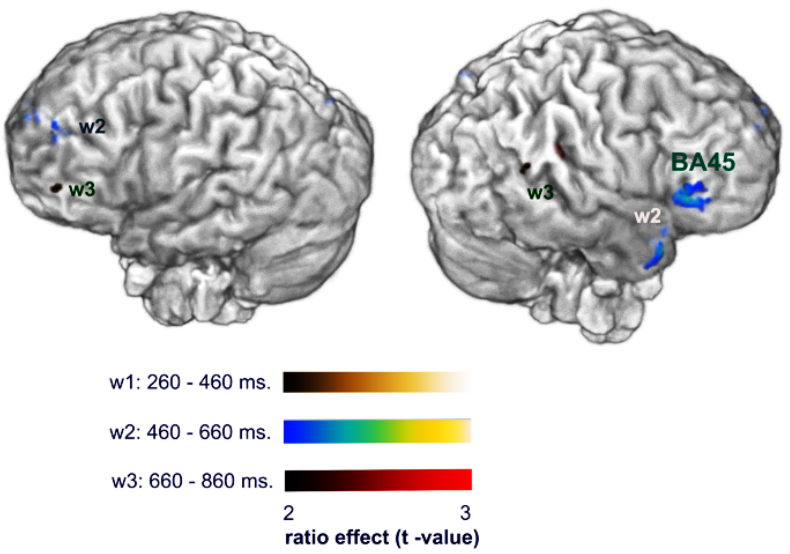

Figure 1. Results at the sensor and at the source spaces. A distributed slow negative wave was observed at the sensor level, which was inversely proportional to the degree of selection demands imposed by the noun condition. The estimated sources based on the timing observed in the ERFs showed the most intense and focal activation in language homologue areas on the right hemisphere (BA 45). This effect was based on a negative correlation with the computed ratios (i.e. selection demands).

\section{Discussion}

MEG showed very clear ERFs effects at the single case as well as highly focal localization upon MNE source estimation. Our adaptation of the pervasively used verb generation paradigm to this patient, precisely revealed language lateralization in this left-handed, left hemisphere epilepsy case (Jokeit et al., Seidenberg et al., 1997). We believe that the easy methodological variation of the verb generation task proposed here offers a conclusive mapping before surgery. It should successfully cue on lateralization and on specific language processes, such as the ability to retrieve the appropriate word from memory, essential to spare language during surgery. The application to further cases is however necessary before drawing firm conclusions.

\section{References}

Bizzi A., Blasi V., Falini A., Ferroli P., Cadioli M., Danesi U., et al. (2008). Presurgical functional MR imaging of language and motor functions: validation with intraoperative 
electrocortical mapping. Radiology, 248(2), 579-89.

De Witte, E., Mariën P. (2013) The neurolinguistic approach to awake surgery reviewed. Clinical Neurology and Neurosurgery, 115, 127-145.

Jokeit H., Ebner A., Holthausen H., Markowitsch H.J., Tuxhorn I. (1996). Reorganization of memory functions after human temporal lobe damage. Neuroreport, 7(10), 1627-1630.

Papagno C., Gallucci M., Casarotti A., Castellano A., Falini A., Fava E., et al. (2011). Connectivity constraints on cortical reorganization of neural circuits involved in object naming. Neuroimage, 55(3), 1306-13.

Rosenberger L.R., Zeck J., Berl M.M., Moore E.N., Ritzl E.K., Shamim S., Weinstein S.L., et al. (2009) Interhemispheric and intrahemispheric language reorganization in complex partial epilepsy. Neurology 72(21), 1830-1836.

Roux FE, Boulanouar K., Lotterie J.A., Mejdoubi M., LeSage J.P., Berry I. (2003) Language functional magnetic resonance imaging in preoperative assessment of language areas: correlation with direct cortical stimulation. Neurosurgery, 52(6), 1335-47.

Seidenberg M., Hermann B.P., Schoenfeld J., Davies K., Wyler A., Dohan F.C. (1997). Reorganization of verbal memory function in early onset left temporal lobe epilepsy. Brain Cognition, 35(1), 132-148.

Thompson-Schill, S.L., D'Esposito, M., Aguirre, G.K., \& Farah, M. J. (1997). Role of left inferior prefrontal cortex in retrieval of se- mantic knowledge: A reevaluation. Proceedings of the National Academy of Sciences, 94, 14792-14797.

Thompson-Schill, S.L., Swick, D., Farah, M.J., D'Esposito, M., Kan, I.P., \& Knight, R.T. (1998). Verb generation in patients with focal frontal lesions: A neuropsychological test of neuroimaging findings. Proceedings of the National Academy of Sciences, 95, 1585515860 . 


\title{
Investigating regular and irregular morphology in Parkinson's and Alzheimer's disease: evidence from Slovenian
}

\author{
Georgia Roumpea $^{1}$, Maja Blesić ${ }^{2}$, Dejan Georgiev ${ }^{3}$, Christina Manouilidou ${ }^{1,3}$ \\ ${ }^{1}$ Department of Comparative and General Linguistics, University of Ljubljana, Slovenia \\ ${ }^{2}$ Mei:CogSci, Faculty of Education, University of Ljubljana, Slovenia \\ ${ }^{3}$ Department of Neurology, University Medical Centre, Ljubljana, Slovenia
}

\section{Introduction}

We investigated the ability of Slovene-speaking individuals diagnosed with Alzheimer's (AD) and Parkinson's (PD) disease to produce regular and irregular forms of verbs and nouns. Regular and irregular forms have occupied the literature of clinical linguistics for quite some time. Ullman et al. (1997) proposed the Declarative/Procedural Model (D/PM) according to which declarative memory is connected with the process of lexical information and production of irregular forms, while procedural memory is responsible for processing grammatical rules and producing regular forms. Ullman et al. (1997), Cortese et al. (2006) and Walenski et al. (2009) found English-speaking and Italian-speaking AD individuals to be impaired in producing irregular forms (taught) compared to regular ones (played), while irregular forms were better preserved in PD. However, other studies failed to replicate the D/PM. Terzi et al. (2005) and Colman et al. (2009) report that Greek-speaking and Dutch-speaking PD individuals showed no dissociation between regular and irregular verbs. Finally, Dick et al. (2018) found PD individuals' syntactic abilities not impaired in a sentence-production and story-retelling task.

While the production of regular and irregular forms has been examined widely in other languages, to our knowledge there is no evidence from Slavic languages where the issue of irregularity is manifested in multiple domains. Our study aims to further investigate the production of regular and irregular morphology as manifested in nominal (singular vs. plural) and verbal inflection (present tense) and as well as aspectual formation. In Slovene, the regular formation of these categories is either by suffixation or by prefixation (Herrity, 2015). While present tense and number are regularly formed by suffixation (e.g. delati $i_{\text {inf }}-$ delam $_{1 \text { sing }}$ "to work -I work", miza $_{\text {sing }}-$ mize $_{\mathrm{pl}}$ "table -tables"), in the formation of aspect, the corresponding perfective form of an imperfective infinitive is usually formed by prefixation (e.g. risati imperf $_{\text {-narisati }}$ perf "to draw -to finish drawing"). However, irregular forms of verbal and nominal inflection as well as aspect are also observed, such as as "iti $i_{\text {inf }}-$ grem $_{1 \text { sing }}$ "to go $-\mathrm{I}$ go", človek $\mathrm{sing}_{\text {sing }}-$ ljudje $_{\mathrm{pl}}$ "human - humans" and metati $i_{\text {imperf }}$-vreči $i_{\text {perf }}$ "to throw -to finish throwing", respectively. Based on the DPM, AD individuals are expected to have difficulties in producing irregular forms due to declarative memory limitations, while PD individuals will perform low in regular forms due to procedural memory impairment. Differences among the categories of verbal and nominal inflection as well as aspectual formation might arise.

\section{Methods}

\section{Participants}

$6 \mathrm{mAD}$ (all female), 5 no-dementia PD (all male) and 5 healthy individuals participated in the study. Participants were matched when it comes to age and years of education [mean age: MCI 77.6, AD 82.5, controls 73.6; mean years of education: MCI 13.2, AD 12.3, controls 12.8). The Mini Mental State Examination (MMSE) was administered to all participants to collect more information about their cognitive profile (MMSE mean score: PD 27, AD 19.5, controls 28.2).

An off-line sentence completion task was designed. Our stimuli consist of 23 verbs (11 regulars, 
12 irregulars and 6 nouns (3 regulars, 3 irregulars). 29 pairs of sentences were designed from which, 6 tested number, i.e. singular vs. plural (3 regulars, 3 irregulars), 14 tested present tense ( 6 regulars, 8 irregulars) and 9 tested aspect ( 5 regulars, 4 irregulars/ 5 perfective, 4 imperfective). The sentence in (1) is an example of producing regular aspect. Participant were given the variant in (1a) and they had to complete the sentence as in (1b).

(1a) Včeraj smo jedli $i_{\text {imperfective/regular }}$ eno uro. (Yesterday, we were eating for an hour)

(1b) Včeraj smo pojedli $i_{\text {perfective/regular }}$ eni uri. (Yesterday, we ate in an hour)

\section{Results}

Percentages of correct responses for regular and irregular forms for both $\mathrm{PD}$ and $\mathrm{mAD}$ group are presented in Figure 1. mAD group performed significantly lower compared to PD and control $(p<.01$ in all comparisons), while PD participants performed equally well as controls ( $>$.103). Furthermore, no dissociation between regular and irregular forms was observed in both $\mathrm{PD}$ and mAD individuals' perfomance (PD: $p=1.000 ; \mathrm{mAD}: p=.863$ ). Analysis of mAD group's results revealed no statistically significant difference between regular and irregular verbs in tense, aspect and plural ( $p=1.000$, in all comparisons). Concerning the different morphological categories, aspect was found to be significantly more impaired compared to tense and plural $(p=.039$, in all comparisons).

Error analysis revealed that mAD participants had no difficulty in producing the present tense of regular verbs (e.g. delati $i_{\text {inf }}-$ delam $_{1 \text { sing }}$ ) and irregular verbs (iti inf $_{\text {- }}$-grem ${ }_{1 \text { sing }}$ ). In detail, participants were able to use correctly the appropriate inflectional suffix to form the present tense of both regular and irregular verbs. The most frequent mistake in the category of tense was the substitution of the target irregular form with a regular one of a verb that was semantically close to the target one and correctly inflected [e.g. hodim (regular, "I am walking") vs. grem (irregular, "I am going")]. Regarding aspect, in regular verbs mAD individuals tended to produce the imperfective form of the verb instead of the perfective target (napisala perfective $_{\rightarrow}$ pisala $\left._{\text {imperfective }}\right)$. In irregular verbs participants tended either to produce the no-target aspect category $\left(\left(\right.\right.$ jemali $_{\text {imperfective }} \rightarrow$ vzeli $\left._{\text {perfective }}\right)$ or to substitute the target verb with another irregular semantically related verb.

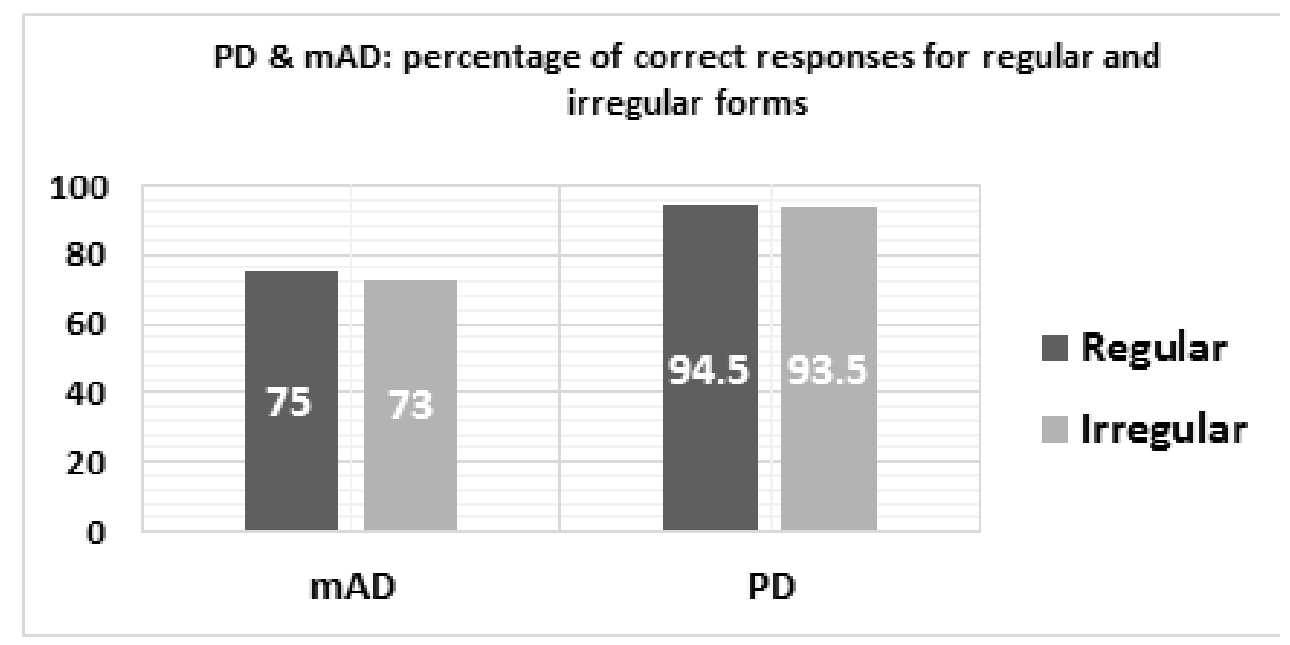

\section{Discussion}

The present study is one of the first attempts to investigate the status of regular and irregular forms in pathological language of Slovene-speaking individuals. PD and mAD individuals 
performed equally in both regular and irregular forms. Our results seem not to support the DPM (e.g. Ullman 2001) and to be in line with previous studies that report no dissociation of regular and irregular forms in PD and AD (e.g. Terzi et al. 2005). Slovene's rich morphology might explain these results. More specifically, grammatical rules are applied not only in regular forms, but in irregular ones as they also manifest agreement (number, gender, case etc.). Also, aspect, which was found to be less preserved compared to number and tense, has already been reported to be impaired in mAD population (Fyndanis et al., 2013; Roumpea et al., 2019). Participants' tendency to use the imperfective form instead of the perfective in regular verbs might also indicate difficulty in producing the aspectual morphology (prefixation). Further research is required to delineate this issue.

\section{References}

Cameli, L., Phillips, N. A., Kousaie, S., \& Panisset, M. (2005). Memory and language in bilingual Alzheimer and Parkinson patients: Insights from verb inflection. In ISB4: Proceedings of the 4th International Symposium on Bilingualism (pp. 452-476).

Colman, K. S., Koerts, J., van Beilen, M., Leenders, K. L., Post, W. J., \& Bastiaanse, R. (2009). The impact of executive functions on verb production in patients with Parkinson's disease. cortex, 45(8), 930-942.

Cortese, M. J., Balota, D. A., Sergent-Marshall, S. D., Buckner, R. L., \& Gold, B. T. (2006). Consistency and regularity in past-tense verb generation in healthy ageing, Alzheimer's disease, and semantic dementia. Cognitive Neuropsychology, 23(6), 856-876.

Dick, J., Fredrick, J., Man, G., Huber, J. E., \& Lee, J. (2018). Sentence production in Parkinson's disease. Clinical linguistics 85 phonetics, 32(9), 804-822.

Fyndanis, V., Manouilidou, C., Koufou, E., Karampekios, S., \& Tsapakis, E. M. (2013). Agrammatic patterns in Alzheimer's disease: Evidence from tense, agreement, and aspect. Aphasiology, 27(2), 178-200.

Herrity, P. (2015). Slovene: A comprehensive grammar. Routledge.

Roumpea, G., Nousia, A., Stavrakaki, S., Nasios, G., \& Manouilidou, C. (2019). Revisiting aspect in Mild Cognitive Impairment and Alzheimer's disease: evidence from Greek (under review). Under review for Frontiers in Human Neuroscience.

Terzi, A., Papapetropoulos, S., \& Kouvelas, E. D. (2005). Past tense formation and comprehension of passive sentences in Parkinson's disease: Evidence from Greek. Brain and Language, 94(3), 297-303.

Ullman, M. T. (2001). A neurocognitive perspective on language: The declarative/procedural model. Nature reviews neuroscience, 2(10), 717.

Ullman, M. T., Corkin, S., Coppola, M., Hickok, G., Growdon, J. H., Koroshetz, W. J., \& Pinker, S. (1997). A neural dissociation within language: Evidence that the mental dictionary is part of declarative memory, and that grammatical rules are processed by the procedural system. Journal of cognitive neuroscience, 9(2), 266-276.

Walenski, M., Sosta, K., Cappa, S., \& Ullman, M. T. (2009). Deficits on irregular verbal morphology in Italian-speaking Alzheimer's disease patients. Neuropsychologia, 47(5), 1245-1255. 


\title{
Semantic memory tasks for neurogenic disorders: Data on healthy elderly adults, Alzheimer's disease and stroke aphasia
}

\author{
Ida Luotonen, Kati Renvall,Pirjo Korpilahti \\ Department of Psychology and Speech-Language Pathology, University of Turku, Finland
}

\section{Introduction}

Semantic memory includes knowledge of the meaning of words and objects (Rogers et al., 2015). Patients with Alzheimer's disease (AD) and aphasia often show difficulties in semantic memory tasks such as picture naming, semantic association tasks and semantic verbal fluency tasks (SVF). Little is known how semantic deficits differ between different neuropathological conditions (Corbett et al., 2009; Libon et al., 2013).

In this study, we used eleven new semantic tasks and two semantic verbal fluency tasks to assess semantic processing across different input and output modalities, types of semantic judgement and number of items. In this presentation, we compare the performance of healthy elderly adults, patients with AD and patients with stroke aphasia in four of the semantic association tasks and two semantic verbal fluency tasks. Within the association tasks, we aim to explore the effect of number of items and modality in semantic processing in all groups.

\section{Methods}

\section{Participants}

The participants consisted of 103 elderly adults who volunteered to participate in this study:

1) Patients with mild to moderate Alzheimer's disease ( $n=20 ; M=74.13$ years; Mini-Mental State Examination (MMSE) $M=23.35$ )

2) Patients with aphasia $(n=23 ; M=68.52$ years; Western Aphasia Battery (WAB) aphasia quotient $M=65.23$ ).

3) Healthy elderly individuals ( $n=60 ; M=68.4$ years; MMSE $M=29.07$ )

Comparing the age and education, we found statistically significant differences between the groups by age $(F(2,100)=7.848, p=.001)$ and by education $(F(2,100)=3.874, p=.024)$.

\section{Assessment and analysis}

All subjects were administered four semantic association tasks similar to PPT (Pyramids and Palm Trees Test; Howard \& Patterson, 1992) and two semantic verbal fluency (SVF) tasks including animal and items of clothing categories. The association tasks $(1+2$ pictures, $1+5$ pictures, $1+2$ words, $1+5$ words) differ between the number of response choices (2 vs. 5$)$ to explore whether the number of items affects the performance. The stimuli are either pictures or words to examine whether modality affects the performance. To compare the mean number of correct responses and the overall performance time between the groups, we conducted one-way analyses of variance (ANOVA) using $p<0.05$ significance level. To compare the different tasks, a repeated-measures analysis of variance with four within-subjects variables (association tasks) were used for each group.

For the SVF tasks, the analysis is ongoing. The number of correct answers, clusters and switches will be analyzed based on the criteria by Abwender et al. (2001) and Troyer et al. (1997). The SVF performance of the three groups will be compared to the semantic association task performance.

\section{Results}


Healthy elderly adults made only few errors in the semantic association tasks (Figure 1(A)). In all association tasks, both patient groups scored significantly lower in the mean number of correct responses and were significantly slower in the overall performance time than the healthy group (Figure 1). However, there were no differences between the patient groups.
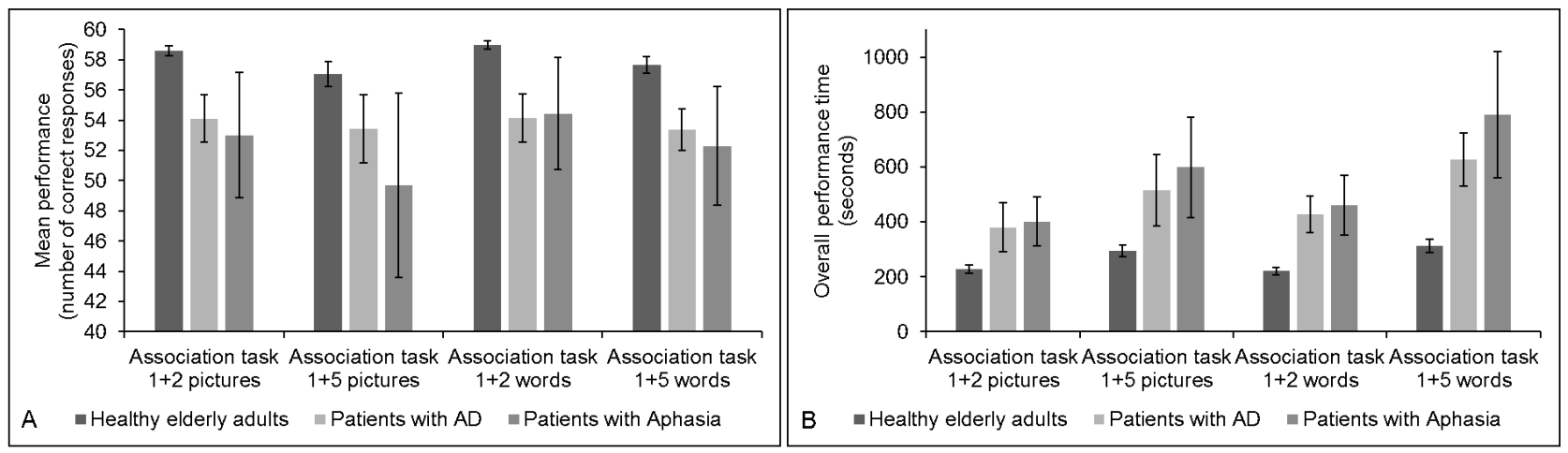

Figure 1. (A) Mean number of correct responses out of 60) and (B) mean overall performance time (seconds) of the three groups in the four semantic association tasks. Error bars represent 95\% CIs.

A one-way repeated measures ANOVA was employed for each group to evaluate the effect of number of items on mean number of correct responses and on mean overall performance time. On the mean number of correct responses, a significant effect of the number of items was found in the healthy elderly group within both task modalities: pictures $F(1,59)=19.740, p<.001$, $\eta \mathrm{p}^{2}=.251$ and words $F(1,59)=27.221, p<.001, \eta \mathrm{p}^{2}=.316$. Also, there was a significant effect of the number of items on the mean number of correct responses in aphasia group with words $F(1,16)=13.607, p=.002, \eta \mathrm{p}^{2}=.460$ but in the AD group no significant effects were found. On the mean overall performance time, a statistically significant effect of the number of items was found in all groups for both modalities (Figure 1(B)).

A one-way repeated measures ANOVA was used to test the effect of input modality. Pictures and written words were compared in the association tasks. In the healthy and the AD group there were no effects of input modality. However, in the aphasia group there was a statistically significant effect of the modality on the overall performance time with $1+2$ items $F(1,17)=$ $11.335, p=.004, \eta \mathrm{p}^{2}=.400$ and with $1+5$ items $F(1,16)=15.314, p=.001, \eta \mathrm{p}^{2}=.489$. The performance was slower with the word stimuli.

\section{Discussion}

Our data showed that there were significant differences between the healthy elderly group and the patient groups in the semantic association tasks. There were no significant differences between the patient groups. The number of items affected the mean number of correct responses with picture and word stimuli in healthy elderly adults and with word stimuli in patients with aphasia. However, the mean number of correct responses of the AD group was not affected by the number of items. The overall performance time was affected by the number of items in all groups. The stimulus modality (pictures or words) only affected the overall performance time in the aphasia group. Results of the SVF tasks and their role in the assessment with the association tasks will be discussed in the conference.

These results suggest that semantic information is processed similarly regardless of the stimulus modality in the healthy group. The role of a semantic task with more related items seems to be unclear in detecting semantic deficits. According to the results, patients with AD might suffer an overall semantic memory loss. In aphasia, the verbal impairment can be accompanied by 
an impairment on non-verbal semantic tasks.

\section{References}

Abwender, D. A., Swan, J. G., Bowerman, J. T., \& Connolly, S. W. (2001). Qualitative Analysis of Verbal Fluency Output: Review and Comparison of Several Scoring Methods. Assessment, 8(3), 323-338.

Corbett, F., Jefferies, E., Ehsan, S. \& Lambon Ralph, M.A. (2009). Different impairments of semantic cognition in semantic dementia and semantic aphasia: evidence from the non-verbal domain. Brain, 132, 2593-2608.

Howard, D. \& Patterson, K. (1992). The pyramids and palm trees test: a test of semantic access from words and pictures (Thames Valley Test Company, Bury St Edmunds).

Libon, D.J., Rascovsky, K., Powers, J., Irwin, D.J., Boller, A., Weinberg, D., McMillan, C.T. \& Grossman, M. (2013). Comparative semantic profiles in semantic dementia and Alzheimer's disease. Brain, 136, 2497-2509.

Rogers, T.T., Patterson, K., Jefferies, E. \& Lambon Ralph, M.A. (2015). Disorders of representation and control in semantic cognition: Effects of familiarity, typicality, and specificity. Neuropsychologia, 76, 220-239.

Troyer, A.K., Moscovitch, M. \& Winocur, G. (1997). Clustering and switching as two components of verbal fluency: Evidence from younger and older healthy adults. Neuropsychology, 11, 138-46. 


\title{
Frontotemporal dementia: a comparative case study of Greek-speaking individuals with the non-fluent and semantic variants of primary progressive aphasia
}

\author{
Nomiki Karpathiou ${ }^{1,2}$, Maria Kambanaros ${ }^{1}$ \\ ${ }^{1}$ Department of Rehabilitation Sciences, Cyprus University of Technology \\ ${ }^{2}$ Athens Alzheimer's Association
}

\begin{abstract}
Introduction
Frontotemporal Dementia (FTD) is an umbrella term that encompasses degenerative disorders of the frontal and anterior temporal lobes that affect behavior and language. FTD overlaps clinically and pathologically with Primary Progressive Aphasia (PPA). PPA is a degenerative syndrome characterized by progressive loss of language function. The consensus criteria for PPA recognize three variants: the non-fluent/agrammatic variant of PPA (nfvPPA), the semantic variant of PPA (svPPA) and the logopenic variant of PPA (lvPPA) (Gorno-Tempini et al., 2011). Each PPA variant has a specific profile of language impairment, different distribution of atrophy on neuroimaging and different likelihood of underlying molecular pathology. Typically, nfvPPA is associated with fronto-insular atrophy, svPPA with atrophy of the anterior and inferior temporal lobe and lvPPA with atrophy of temporo-parietal regions. The most common types of neurodegeneration in PPA are frontotemporal lobar degeneration and Alzheimer's disease (Spinelli et al., 2017). FTD includes two out of three PPA variants, the nfvPPA and svPPA, as the most typical pathology of these variants is frontotemporal lobar degeneration.
\end{abstract}

PPA is characterized by a more partial and progressive pattern of damage than stroke-induced aphasia and targets areas such as the anterior temporal lobe that are rarely affected by stroke (Mesulam, 2016). Clinical and neuroimaging research on PPA has advanced our understanding of the language network. It has shown, for example, that the left anterior temporal lobe plays a critical role in single word comprehension and object naming and that the traditional 'Wernicke's area' is important for language repetition and sentence comprehension but not single word comprehension (Mesulam et al., 2019).

The aim of this study is to compare the clinical presentation of the language variants of FTD, nfvPPA and svPPA, in two Greek-speaking individuals with PPA. Greek is an underrepresented language in the literature on PPA research. It is a highly inflected and stem-based language. To this end, a comprehensive battery of neuropsychological tests and narrative analysis was employed.

\section{Methods}

Participants

Two individuals diagnosed with PPA and 12 neurologically healthy adults participated in this study. All participants were right-handed.

The participant with the non-fluent variant of PPA, is a 61-year-old man with 6 years of formal education. He was assessed five years after symptom onset. He then had a sum of boxes score of 9 on the FTLD-modified Clinical Dementia Rating (CDR). MRI demonstrated left perisylvian atrophy.

The participant with the semantic variant of PPA is a 73-year-old man with 9 years of formal education. At the time of the study he had 5 years into the disease and a FTLD-modified CDR sum of boxes score of 6 . His MRI scan showed the typical pattern of asymmetric anterior 
temporal lobe atrophy.

The control group consisted of 2 male and 10 female native Greek speakers with a mean age of $68.08(\mathrm{SD}=5.52)$ years and a mean of $13(\mathrm{SD}=3.19)$ years of education.

\section{Procedure}

Participants were evaluated using a battery of tests assessing executive function, memory, visuospatial abilities, object semantics, mood, praxis, motor speech abilities, single word and sentence comprehension, repetition, confrontation naming, reading, writing and connected speech production. Quantitative production analysis (QPA) (Saffran et al., 1989) was used for the narrative analysis of a story retell task.

Neuropsychological testing was completed in four 45-minutes-sessions for the participants with PPA and three sessions for the control participants. MRI scans and reports were made available for the two individuals with PPA.

\section{Statistical analysis}

Crawford and Howell's method was used to compare performance of each subject with that of the control sample (Crawford, Garthwaite \& Porter, 2010). T values were also calculated to compare the scores of the two subjects with reference to the control sample (Crawford, Garthwaite \& Wood, 2010).

\section{Results}

The participant with the nfvPPA performed worse than the participant with the svPPA on the Digit Span -reverse recall task $(p=0.025)$, Clock Drawing Test $(p<0.001)$, syntactic comprehension (Boston Diagnostic Aphasia Examination, BDAE-3, $p=0.014$ ) and reading fluency for words $(p<0.001)$.

The participant with the svPPA was more impaired in confrontation naming (Boston Naming Test-15, $p<0.001$ ), single word comprehension (Peabody Picture Vocabulary Test, $p<0.001$ ) and object semantics (Pyramid and Palm Trees Test, $p=0.001$ ). Comprehension of auditory complex material, written words and sentences were affected $(p=0.022, p=0.005$ and $p<0.001$, respectively), although his ability to follow commands was within normal limits and performance for syntactic comprehension was at ceiling. Both participants were impaired in the Trail Making Test A and B, verbal fluency, spelling and written picture description.

The narrative production measures that differed significantly between the two participants were speech rate (slower for the nfvPPA participant, $p=0.007$ ), average pause duration (longer for the nfvPPA participant, $p<0.001$ ), false starts per min (more for the nfvPPA participant, $p=0.045$ ), proportion of nouns (lower for the svPPA participant, $p=0.012$ ) and closed class words (lower for the nfvPPA participant, $p=0.016)$. Compared to the control group, the nfvPPA participant produced shorter sentences $(p=0.023)$, fewer closed class words $(p=0.006)$, made longer pauses $(p<0.001)$ and spoke at a slower rate $(p<0.001)$. The svPPA participant used fewer nouns $(p=0.027)$, more pronouns $(p=0.02)$ and fewer narrative words as a proportion of the total words produced $(p=0.003)$.

\section{Discussion}

The results confirm the distinctive features of both PPA variants, namely anomia, a single word comprehension deficit, preserved repetition and syntactic comprehension for the participant with the svPPA, as well as motor speech and syntactic processing difficulties alongside 
with intact repetition, semantic knowledge and naming ability for the nfvPPA participant.

Taking into account the neuroimaging findings, these two cases illustrate the different distribution of atrophy in the language variants of FTD and highlight the role of the left anterior temporal lobe in naming and single word comprehension.

\section{References}

Gorno-Tempini, M. L., Hillis, A. E., Weintraub, S., Kertesz, A., Mendez, M., Cappa, S. F., ... \& Manes, F. (2011). Classification of primary progressive aphasia and its variants. Neurology, 76(11), 1006-1014.

Spinelli, E. G., Mandelli, M. L., Miller, Z. A., Santos-Santos, M. A., Wilson, S. M., Agosta, F., ... \& Henry, M. L. (2017). Typical and atypical pathology in primary progressive aphasia variants. Annals of neurology, 81(3), 430-443.

Mesulam, M. (2016). Primary progressive aphasia and the left hemisphere language network. Dementia and neurocognitive disorders, 15(4), 93-102.

Mesulam, M. M., Rader, B. M., Sridhar, J., Nelson, M. J., Hyun, J., Rademaker, A., ... \& Weintraub, S. (2019). Word comprehension in temporal cortex and Wernicke area: A PPA perspective. Neurology, 92(3), e224-e233.

Saffran, E. M., Berndt, R. S., \& Schwartz, M. F. (1989). The quantitative analysis of agrammatic production: Procedure and data. Brain and language, 37(3), 440-479.

Crawford, J. R., Garthwaite, P. H., \& Porter, S. (2010). Point and interval estimates of effect sizes for the case-controls design in neuropsychology: rationale, methods, implementations, and proposed reporting standards. Cognitive neuropsychology, 27(3), 245-260.

Crawford, J. R., Garthwaite, P. H., \& Wood, L. T. (2010). Inferential methods for comparing two single cases. Cognitive neuropsychology, $27(5), 377-400$. 


\title{
The role of the uncinate fasciculus and inferior longitudinal fasciculus in healthy and disordered language production
}

\author{
Nikki Janssen ${ }^{1,2}$, Margot Mangnus ${ }^{1}$, Ardi Roelofs ${ }^{1}$, Joanna Sierpowska ${ }^{1}$, Roy Kessels ${ }^{1,2}$, \\ Vitória Piai ${ }^{1,2}$ \\ ${ }^{1}$ Donders Institute for Brain, Cognition and Behavior, Nijmegen \\ ${ }^{2}$ Department of Medical Psychology, Radboud University Medical Center, Nijmegen, the \\ Netherlands
}

\section{Introduction}

Primary progressive aphasia (PPA) is an acquired language deficit due to progressive neurodegenerative disease, with word retrieval disturbances as the most frequent presenting symptom (Mesulam, 2001). The picture-word interference (PWI) paradigm has been extensively employed in the field of psycholinguistics to investigate word retrieval and top-down control (Piai, Riès \& Swick, 2016), yet very few studies have used this paradigm in PPA. Moreover, in combination with white matter tractography, the PWI paradigm could shed light on the involvement of the ventral white matter pathway, as the exact functional role of this pathway in speech production is much debated. The ventral pathway has been suggested to underlie top-down control in language production (Roelofs, 2014), but the functional role of each specific white matter tract within this pathway, like the uncinate fasciculus (UF) and the inferior longitudinal fasciculus (ILF), has not yet been elucidated. Here we investigate top-down interference control in individuals with PPA through the PWI paradigm, and asses the possible relationship between this interference control and the microstructural properties of the UF and ILF.

\section{Methods}

\section{Sample and Task}

To investigate the PWI paradigm in PPA and the involvement of the UF and ILF in top-down interference control, seventeen individuals with PPA and twenty-two age-matched healthy controls (HC) performed a PWI task including an incongruent, semantically related word (e.g. written word "cow" superimposed on the picture of a horse) and a neutral stimulus ("XXX" on the picture of a horse). The picture stimuli consisted of colored photographs of 12 high frequency words from two semantic categories (animals and fruits). The difference between semantically related and neutral picture-word pairs (the PWI effect) in both reaction time (RT) and accuracy served as a behavioral measure of the participants' top-down interference control. Analyses were run with the lme4 package for R (Bates \& Maechler, 2009).

\section{Diffusion weighted imaging analyses}

DWI was acquired with a simultaneous-multislice diffusion-weighted Echo Planar Imaging (EPI) sequence with the following parameters: multiband factor $=3$, TR/TE $=2282 / 71.2 \mathrm{~ms}$, in-plane acceleration iPAT $=2$, voxel size $=2 \times 2 \times 2 \mathrm{~mm} 3,9$ unweighted scans, 100 diffusionencoding gradient directions in multiple shells (b-values $=1250$ and $2500 \mathrm{~s} / \mathrm{mm} 2$ ), Taq $=$ $8 \mathrm{~min} 29 \mathrm{~s}$. TRActs Constrained by UnderLying Anatomy (TRACULA) was used to delineate the UF and ILF, for each participant by using global probabilistic tractography with anatomical priors (Yendiki et al., 2011). Then, the microstructural integrity of both the UF and ILF was calculated as a neuroanatomical measure, expressed in Fractional Anisotropy (FA) and Mean Diffusivity (MD) values that were based on the weighted average over the entire support of the path distribution. Between group comparisons of microstructural properties were performed using non-parametric tests (Kruskal \& Wallis, 1952). A principal component analysis was performed on the FA and MD values to summarize the microstructural properties per tract. The relation between these microstructural properties, operationalized as the first principal compo- 
nent, and PWI effects in both RT and accuracy were analyzed with linear mixed-effects model and mixed-effects logistic regression, respectively, both with the same model structure.

\section{Results}

As expected, patients were slower than controls ( $p<0.001$, see Fig 1 , left panel) in both conditions. The top-down interference (related vs. neutral) effect in RT was observed for both PPA patients and controls $(p<0.001)$ and the interference effect was statistically larger for PPA patients than for controls ( $p<0.001$, see Fig. 1, right panel).
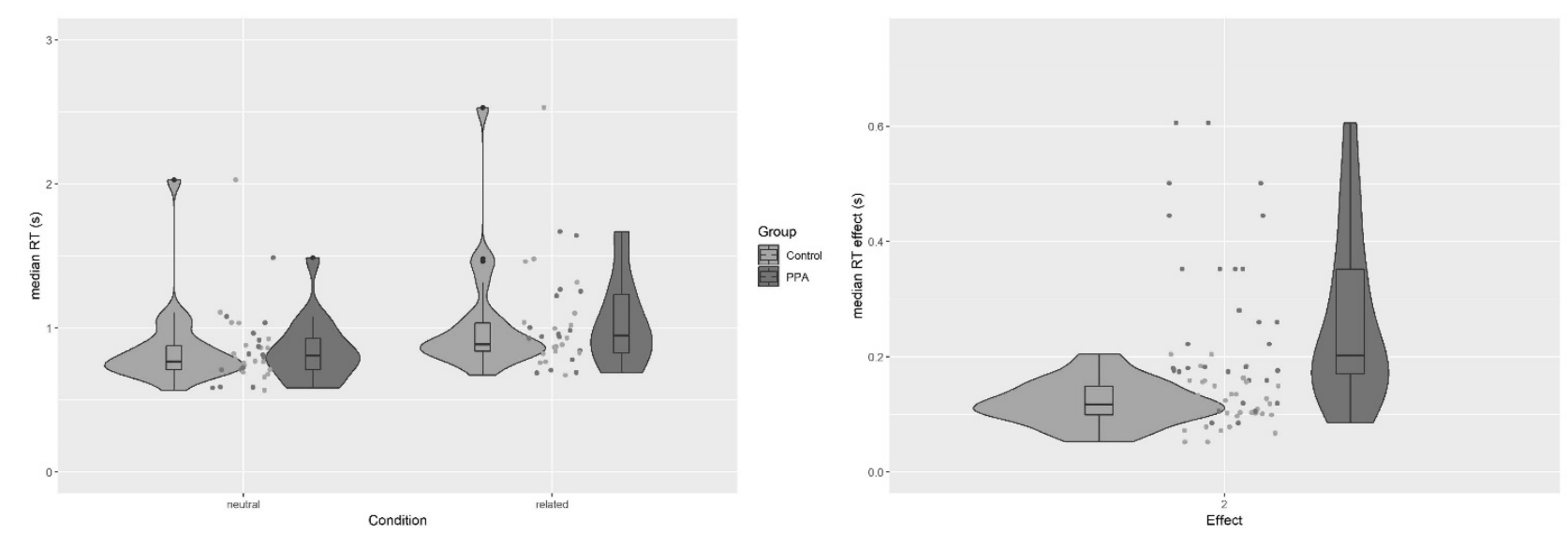

Figure 1. Group comparisons for RT on both task conditions and picture-word interference effect (related -neutral).

Regarding accuracy, PPA patients made more errors compared to controls $(p<0.001)$ and both groups made more errors in the related condition $(p=0.003)$. Moreover, the microstructural properties were altered in patients compared to controls, with PPA patients having lower FA and higher MD values for both the UF $(p<0.001)$ and the ILF $(p<0.001)$. Importantly, microstructural properties of the ILF were related to RT and the amount of errors being made in each condition (both $p<0.001)$ and to the interference effect in RT $(p<0.001)$, but not in accuracy $(\mathrm{p}>0.05)$. Furthermore, microstructural properties of the UF showed a significant relation to accuracy $(p=0.004)$, but not to $\mathrm{RT}(p=0.08)$, and to the interference effect in both RT $(p=0.026)$ and accuracy $(p=0.046)$.

\section{Discussion}

The behavioral results show an increased interference effect in the reaction times of individuals with PPA, as compared to controls, suggesting an impairment in top-down control processes in language production over and above their naming impairments. Furthermore, the ILF appears to be related to RT and accuracy in both conditions, which is in accordance with previous findings suggesting the ILF to be involved in object recognition (Herbet, Zemmoura \& Duffau, 2018). Both UF and ILF are related to the interference effect in RT, while only the UF is related to the interference effect in accuracy. This suggests a ventral pathway involvement in word production related interference. These findings contribute to the scarce literature on this topic and require further investigation.

\section{References}

Bates, D., Maechler, M., \& Bolker, B. (2012). lme4: Linear mixed-effects models using S4 classes. R package version 0.999375-32. 2009.

Herbet, G., Zemmoura, I., \& Duffau, H. (2018). Functional anatomy of the inferior longitudinal fasciculus: from historical reports to current hypotheses. Frontiers in neuroanatomy, 12. 
Kruskal, W. H., \& Wallis, W. A. (1952). Use of ranks in one-criterion variance analysis. Journal of the American statistical Association, 47(260), 583-621.

Mesulam, M. M. (2001). Primary progressive aphasia. Annals of neurology, 49(4), 425-432.

Piai, V., Riès, S. K., \& Swick, D. (2016). Lesions to lateral prefrontal cortex impair lexical interference control in word production. Frontiers in human neuroscience, 9, 721.

Roelofs, A. (2014). A dorsal-pathway account of aphasic language production: The WEAVER ++ / ARC model. Cortex, 59, 33-48.

Yendiki, A., Panneck, P., Srinivasan, P., Stevens, A., Zöllei, L., Augustinack, J., ... \& Jbabdi, S. (2011). Automated probabilistic reconstruction of white-matter pathways in health and disease using an atlas of the underlying anatomy. Frontiers in neuroinformatics, 5, 23. 


\section{Poster Session IV}




\title{
Effect of Type of Language Therapy on Fluency in Patients with Post-Stroke Aphasia
}

\author{
Saša R. Filipović ${ }^{1}$, Jasmina Vuksanović ${ }^{1}$, Tanja Milovanović ${ }^{2}$ Ljubica Konstantinoviće,3 \\ ${ }^{1}$ University of Belgrade, Institute for Medical Research, Dr Subotica 4, Belgrade, Serbia \\ ${ }^{2}$ Klinika za Rehabilitaciju "Dr Miroslav Zotović, Sokobanjska 13, Belgrade, Serbia \\ ${ }^{3}$ University of Belgrade, Faculty of Medicine (Rehabilitation), Dr Subotica 8, Belgrade, Serbia
}

\section{Introduction}

Although various speech and language therapy (SLT) approaches have been developed, the most effective strategies of treating aphasia after stroke have to be determined yet (Brady et al., 2016). Standard aphasia therapy (SAT) is one of the most frequently used therapeutic techniques and allows all modes of communication, both linguistic and extra-linguistic. On the other hand, Constraint-Induced Aphasia Therapy (CIAT) approach, based on constraint usage of the language channel only, massed practice, and shaping through therapeutic language games, has been suggested as a more efficient than traditional therapy approaches (Pulvermüller, et al., 2008; Berthier, \& Pulvermüller, 2011). When considering CIAT success in language recovery after stroke, there is still an open debate which factors are at play: is maximisation of quantity and frequency of language therapy the most relevant factor affecting therapy outcome or it is the focusing on language through constraints. In this paper, we examine verbal semantic fluency (VSF) performance in a subset of treated aphasic patients who participated in previously reported research (Vuksanovic et al., 2018). As a measure of expressive lexical recovery SVF performance was used for two main clinical purposes -to assess the degree of lexico-semantic system impairment and cognitive processes underlying successful lexical production, and its role as a predictor of language improvement over time in aphasic patients, because it relies on both linguistic and cognitive processes.

\section{Methods}

Procedure

Patients in rehabilitation received two successive four-week blocks of intense (one and a half hour per day, five days a week) aphasia therapy in a randomized within-subject crossover design. One therapy block (a total of 30 hours each) consisted of SAT, another of CIAT. Thus, the total amount of therapy was the same for all patients. Therapy protocols for all patients were delivered by a single experienced speech-language therapist. The examiner was blinded for the order and the type of therapy administered, while the therapist was blinded for the results of the measured variables.

\section{Subjects}

A total of 20 consecutive right-handed patients with non-fluent post-stroke aphasia of up to 1 year duration were randomly assigned to two treatment groups. One group had SAT first followed by CIAT (S1C2 group), while another had a reversed order of the therapies, CIAT first followed by SAT (C1S2 group). For all participants, diagnosis of post-stroke aphasia was made by neurologists and confirmed by speech therapists based on their assessment, which included the Boston Diagnostic Aphasia Examination (BDAE; Goodglass and Kaplan 1983). The participants and their family members gave their informed consent for the participation in the study. The study was conducted according to the Declaration of Helsinki and approved by the institution Ethics committee.

Subjects were instructed to generate as many words as possible belonging to a certain semantic 
category: wild animals and domestic animals in a period of one minute for each category. All transcripts were analysed for correct responses, repetitions, and errors. In line with common practice in scoring SVF data, only real words were counted. Errors were defined as follows: perseverations, derivations (adding a plural marker or other morpheme to a previously named correct item), intelligible words that are not members of the category currently being tested, and pseudo-words.

\section{Results}

The results of a 2 (group) x 4 (time) two-way mixed ANOVA conducted on the SVF component score yielded significant effects the factor TIME F $(3,15)=38.54, p=.000, \eta^{2}=.68$, but no significant effect the factor GROUP F $(1,15)=4.23, p=.054, \eta^{2}=.19$, and no the TIME $\times$ GROUP interaction $\mathrm{F}(3,15)=3.37, \mathrm{p}>.05, \eta^{2}=.16$. The results indicate that the pattern of improvement is significant during therapy in both groups regardless of the type of the therapeutic approach and a certain time interval of exposure. It should be noted that the factor Group, although not significant, is approaching significance. Furthermore, number of repetitions was higher along with SVF improvement in both groups during the language rehabilitation process.

\section{Discussion}

Although caution should be exercised in making generalizations from the data obtained in our study, generally taken, both CIAT and CAT when applied with equal intensity seem to be efficacious methods in SVF recovery for sub-acute aphasia patients. In this sense it is likely that by increasing the intensity of therapy, irrespective of the kind of approach, favourable long-term outcomes in lexico-semantic language performance can be achieved. Furthermore, the higher number of repetitions along with SVF improvement in both groups during the language rehabilitation process could be interpreted in line with data obtained in the study done by Bose et al. (2017), which have shown impaired switching between clusters, thus reflecting that some underlying cognitive processes (monitoring, shifting) may not be improved.

\section{References}

Berthier, M. L., \& Pulvermüller, F. (2011). Neuroscience insights improve neurorehabilitation of poststroke aphasia. Nature Reviews Neurology, 7, 86-97.

Bose, A., Wood, R., \& Kiran, S. (2017). Semantic fluency in aphasia: clustering and switching in the course of 1 minute. International Journal of Language $\&$ Communication Disorders, 52(3),334-345.

Brady, M. C., Kelly, H., Godwin, J., Enderby, P. \& Campbell, P. (2016). Speech and language therapy for aphasia following stroke. Cochrane Database Systematic Review, 1, 6: CD000425.

Goodglass, H., and Kaplan, E. (1983). The Assessment of Aphasia and Related Disorders (Philadelphia, PA: Lea \& Febiger).

Pulvermuller , F. and Berthier, M. L. (2008). Aphasia therapy on a neuroscience basis. Aphasiology, 22, 563-599.

Vuksanović, J., Milovanović, T., Konstantinović, LJ., and Filipović, S. (2018). Effect of type of language therapy on expressive language skills in patients with post-stroke aphasia. International Journal of Language and Communication Disorders, 53(4),825-835. 


\title{
Effects of language therapies on receptive language recovery in post-stroke aphasia patients
}

\author{
Tanja Milovanović ${ }^{2}$, Jasmina Vuksanović ${ }^{1}$, Ljubica Konstantinoviće ${ }^{2,3}$, Saša R. Filipović ${ }^{1}$ \\ ${ }^{1}$ University of Belgrade, Institute for Medical Research, Dr Subotica 4, Belgrade, Serbia \\ ${ }^{2}$ Klinika za Rehabilitaciju "Dr Miroslav Zotović", Sokobanjska 13, Belgrade, Serbia \\ ${ }^{3}$ University of Belgrade, Faculty of Medicine (Rehabilitation), Dr Subotica 8, Belgrade, Serbia
}

\section{Introduction}

A growing body of studies indicates that recently developed Constraint-Induced Aphasia Therapy (CIAT) approach can be effective in language recovery in aphasic patients. The theoretical model behind this approach is based on the assumption that the behavior of attempting to speak without success creates communication frustration resulting in fewer speaking attempts, more reliance on compensatory strategies, and less cortical activation of the language areas (Szaflarski et al., 2015; Pulvermuller, and Berthier, 2008). However, there are still open issues: which factors are at play when considering CIAT success: is maximization of quantity and frequency of language therapy the most relevant factor affecting therapy outcome, or the focusing on language through constraints. It is known that brain functional reorganization depends on practice (Hamzei, Liepert, Dettmers, Weiller, \& Rijntjes, 2006), so it is reasonable to conclude that a larger amount of therapy will lead to better functional recovery. Another less known issue regarding comparative efficiency of CIAT is its effect on receptive speech. The CIAT is essentially focused on expressive language skills relevant for improving communicative repertoire in social interactions and studies carried so far have been manly focused at exploring CIAT's effects on expressive language improvement (Maher et al., 2006; Attard et al. 2013). The present study aims to investigate the effect of two factors in SL therapy: the factor of massed daily implementation of SLT, and the constraint usage of language channel only on receptive language recovery in aphasic patients. Accordingly, we examined the comparative efficacy of the CIAT and the standard aphasia therapy (SAT) approach on receptive language recovery in nonfluent aphasic patients during the post-acute stage after stroke.

\section{Methods}

\section{Procedure}

Two successive 4-week blocks of intense (1-hour, 5 days a week) aphasia therapy of each therapy program were delivered in a randomized within-subject crossover design -one therapy block consisted SAT, another of CIAT. Twenty consecutive patients, up to a year after stroke, were randomly assigned to either have SAT followed by CIAT (S1C2 group), or to have CIAT followed by SAT (C1S2 group).

\section{Subjects}

A total of 17 participants right-handed patients with non-fluent post-stroke aphasia completed the full treatment plan. They were randomly assigned to two treatment groups were randomly assigned to two experimental-treatment groups with reversed order of therapies delivery. The S1C2 group had SAT first followed by CIAT, while the C1S2 group had CIAT first followed by SAT. All participants were monolingual native Serbian speakers. For all participants, diagnosis of post-stroke aphasia was made by neurologists and confirmed by speech therapists based on their assessment, which included the Boston Diagnostic Aphasia Examination (BDAE; Goodglass and Kaplan 1983). The participants, and their family members when it was required due to communication difficulties, gave their informed consent for the participation in the study. The study was conducted according to the Declaration of Helsinki. The study was approved by the institution ethics committee. 


\section{Measures}

The BDAE clinical subtests of comprehension: the Word Discrimination, the Body-Part Identification, the Commands, and the Complex Ideational Material subtest, were given at the beginning and at the end of each therapy block, as well as one month after the whole treatment stopped. The outcome measures used for analyzes were scores on the mentioned BDAE subtests as well as the composite Composite Comprehension score based on average of the subtests scores.

\section{Results}

In all time points both groups demonstrated significant improvement, i.e. both rehabilitation therapies has an effect on receptive language recovery when are done within massed practice principle. Additionally, the results of this study provide further proof that the improvements are not transient - the results were stable after a month with no therapy, suggesting that intensive SLT provides long-term stability of improved receptive language performance. However, factor group does not have significant impact on receptive language improvement.

\section{Discussion}

it seems from the results of this study that both CIAT and, more conventional, SAT when applied with equal intensity seem to be efficacious methods in receptive language recovery for aphasic patients within the first post-stroke year. In this sense it is likely that just by increasing the intensity of therapy, regardless of the type of approach, a beneficial effect on brain recovery and plasticity can be achieved paving the way to a favorable long-term outcome in receptive language performance.

\section{References}

Attard, M., Rose, M., \& Lanyon, L. (2013). The comparative effects of multi modality aphasia therapy and constraint induced aphasia therapy-plus for severe chronic Broca's aphasia: An in-depth pilot

Goodglass, H. and Kaplan, E. (1983). The Assessment of Aphasia and Related Disorders (Philadelphia, PA: Lea \& Febiger).

Hamzei, F., Liepert, J., Dettmers, C., Weiller, C., Rijntjes, M. (2006).Two different reorganization patterns after rehabilitative therapy: an exploratory study with fMRI and TMS. Neuroimage, 31,710-720.

Maher, L. M., Kendall, D., Swearengin, J. A., Rodriguez, A., Leon, S. A., Pingel, K., ... Rothi, L., J., G. (2006). A pilot study of use-dependent learning in the context of Constraint Induced Language Therapy. Journal of the International Neuropsychological Society, 12(6), 843-852.

Pulvermuller , F. and Berthier, M. L. (2008). Aphasia therapy on a neuroscience basis. Aphasiology, 22, 563-599.

Szaflarski, J.P., Ball, A.L., Vannest, J., et al. (2015). Constraint-Induced Aphasia Therapy for Treatment of Chronic Post-Stroke Aphasia: A Randomized, Blinded, Controlled Pilot Trial. Med Sci Monit., 21, 2861-9. 


\title{
RELEASE-ing the potential of a large, international, systematic review-based Individual Participant Data (IPD) aphasia after stroke database for meta- and network meta-analysis
}

\author{
Myzoon Ali on behalf of the RELEASE Collaborators \\ NMAHP Research Unit, Glasgow Caledonian University, Glasgow, UK
}

\section{Introduction}

Aphasia impacts on societal participation, activities of daily living, emotional wellbeing, and returning home and to work. Effective rehabilitation is vital. The existing evidence base for aphasia therapies has arisen from many small-scale randomised controlled trials and other research studies which inform systematic reviews and meta-analyses of aggregated summary data. While these group-level statistics provide important overviews of the field, this approach also carries a risk of ecological bias and confounding which should be carefully considered.

Analysis of a large aphasia IPD dataset would permit exploration of a highly heterogeneous population, individual level covariates' influence on rehabilitation effects across language modalities and to control for individualistic predictors. Feasibility is contingent on the number of datasets and IPD available. In this presentation we aim to share our methodological approach to establishing a large, international, IPD dataset relating to aphasia after stroke and our preparation of the data in advance of meta-analysis and network meta-analysis.

\section{Methods}

\section{Information sources}

We undertook a systematic literature search (PROSPERO: CRD42018110947) for eligible datasets that included IPD on 10 or more people with aphasia, their time since stroke and a measure of language impairment. Data which were qualitative, based on non-stroke participants or where IPD language data was unavailable were excluded. MEDLINE, AMED, CINAHL, Cochrane Library Databases (CDSR, DARE, CENTRAL, HTA), LLBA, EMBASE, and SpeechBITE along with major trials registers were systematically searched from inception to September 2015. Contributions were also invited from members of the EU-funded Collaboration of Aphasia Trialists (CATs). Where relevant, we evaluated risk of selection, performance, detection and attrition bias at primary research level. We also considered the risk of metabiases including availability and publication bias.

\section{Data extraction}

For each dataset, extracted IPD included individual, aphasia and stroke profiles, and intervention characteristics using the Template for Intervention Description and Replication (TIDiER) checklist (Hoffmann 2014). Two researchers independently extracted information on participants' demography and speech and language therapy (SLT) intervention descriptions for entry into the RELEASE database. Information on therapy approaches, materials and procedures were extracted as direct quotes from published reports or researchers. Using the narrative descriptions, similar approaches were grouped and assigned to one or more category labels by an experienced therapist. Preliminary categories were shared with RELEASE Collaborators for review. Categories were not mutually exclusive; rather they represented different ways of categorising complex interventions. Group discussion and edits on the proposed categorisation followed until consensus had been achieved.

We used an algorithm to aggregate disparate outcome data within the common domains of 
language measurement (overall language ability, auditory comprehension, spoken language production (including naming), reading, writing and functional communication to prevent loss of clinical relevance. For each domain we profiled language measurements used (number of studies using that measure, total IPD available, median, interquartile range, minimum and maximum for each measure). Language or version variations were treated as separate measures. The measurement used in the most datasets for each domain was identified as the "anchor measure". Where two or more measures were used by a similar number of datasets, we applied a systematic approach to prioritising a measure. All other measures within the domain, were considered "minority measures".

\section{Transformation}

A linear transformation from each minority measure to the anchor measure was applied to each quartile and repeated for each language outcome. Each threshold in the minority instrument was mapped to the corresponding threshold in the anchor measure. Values in the minority measure that fell within a quadrant were mapped to the anchor measure relative to the distance from the quadrant edges. The formula was repeated for each quadrant. In this way the clinical relevance of change scores on the anchor measure was retained. Checks were performed to assess whether the ranges, values and directions of changes observed for each anchor measure were consistently reflected when transforming each minority measure into the anchor measure.

\section{Random or fixed effects}

A key decision in any meta-analysis is whether to use a random or fixed effect model in the meta-analysis. Standard errors for random effects models are more efficient, and thus we used a random effects models $(\mathrm{Wu}, 1973)$. We used the Wu-Hausman test to assess whether the effects were consistent across both approaches and do not preclude the use of random effects.

\section{Results}

We received or extracted 5,928 IPD from 174 aphasia studies conducted across 28 countries, published between 1973 and 2017. A quarter of the primary research datasets included were RCTs (45/174 datasets (25.9\%); 1766/5928 IPD (29.8\%)). The remainder were case series or cohort studies (105/174 (60.3\%); 2888/5928 (48.7\%)) and non-RCTs $(19 / 174(10.9 \%) ; 421 / 5928$ $(7.1 \%))$. Five datasets were derived from registries (5/174 (2.9\%); 853/5928 (14.4\%)). Approximately half the datasets included an SLT intervention (91/174 (52.3\%); 2746/5928 (46.3\%)) while the remainder were non-interventional in nature.

Median participant age was 63 years (IQR 53 to 72 ) and $61.3 \%$ were male (3402/5928 IPD). Years of formal education was recorded in fewer than half of datasets (85/174 datasets (48.9\%); $3129 / 5928$ IPD (52.8\%)). Where reported, the participants had a median of 12 (IQR 10 to 16) educational years. Where ethnicity was reported, three-quarters of participants were from a White ethnic background (1116/1475 IPD (75.7\%); data on other ethnic groups were sparse.

Data on overall language ability was captured using 21 different measures; auditory comprehension using 35 measures, naming using 33 measures, reading using 21 measures, writing using 18 measures, and functional communication using 16 outcome measures.

We categorised data into 15 SLT approaches. Therapy interventions were categorised based on three perspectives (a) the role of the intervention within the study design e.g. usual care as a comparison control (b) the intervention target e.g. rehabilitation of spoken language production and (c) the theoretical approach e.g. semantic therapy. Inadequate reporting of therapy interventions, procedures and materials hampered some classifications. 


\section{Discussion}

Collaborative efforts created a large, international IPD aphasia after stroke database which will inform our understanding of language recovery and the components of effective therapeutic interventions. Heterogeneity continues to be evident in the reporting of demographic and clinical data in aphasia studies.

\section{References}

Hoffmann, T. C., Glasziou, P. P., Boutron, I., Milne, R., Perera, R., Moher, D. et al (2014). Better reporting of interventions: template for intervention description and replication (TIDieR) checklist and guide. BMJ (Clinical Research Ed.), 348, g1687. https://doi.org/10.1136/bmj.g1687

Wu, D-M. (1973). Alternative tests of independence between stochastic regressors and disturbances. Econometrica, 41(4), 733-750. 


\title{
Effects of orthographic depth on functional connectivity within reading pathways in proficient bilinguals
}

\author{
Pauliina Sorvisto, Paul Mullins, Marie-Josèphe Tainturier \\ Bangor University, UK
}

\section{Introduction}

Background: It has been suggested that word reading occurs along two main anatomical pathways: the dorsal stream implicated in phonology and the ventral stream engaged in lexicosemantic processing. There is cross-linguistic evidence that the recruitment of the ventral stream is stronger in languages with a deeper orthography such as English. In contrast, the recruitment of the dorsal pathway is more prominent in more transparent languages with more consistent letter-to-sound correspondence (e.g. Das et al., 2011; Richlan, 2014). An interesting question is what happens in bilingual readers of languages with different orthographic depth. Would the cross-linguistic patterns also apply or would reading bilingually lead to a more unified system for the two languages? Oliver, Carreiras and Paz-Alonso (2017) compared fMRI activation and functional connectivity while reading for meaning in Spanish speakers that had either English or transparent Basque as a second language. They observed that Basque L2 readers showed greater coactivation within the dorsal network while English L2 readers showed greater coactivation within the ventral network. This is consistent with cross-linguistic results. However, reading bilingually from an early age may lead to a higher convergence in written word comprehension processes.

Aims:

The goal of the current study was to examine the relative involvement of the ventral vs dorsal pathways in proficient early bilingual readers of two languages with highly contrasted orthographic depth: English vs Welsh. In previous GLM analyses, we showed comparable patterns of activation in the two languages. However, MVPA analyses revealed language sensitivity in several language regions. In this study, we examined if English and Welsh are associated with different patterns of functional connectivity in the ventral/dorsal streams.

\section{Methods}

While being scanned for fMRI, 20 proficient Welsh-English early bilingual adults performed a semantic categorisation task (natural vs man-made) on 192 Welsh and 192 English written, non-cognate translation equivalent nouns presented in alternated blocks separated by rest blocks. We performed psycho-physiological interactions (PPI) analyses for each language using the posterior visual word form area (pVWFA) as the seed region as it is engaged in the earliest stages of written word processing in alphabetic systems (Bouhali et al., 2014). The functional and structural images were preprocessed using SPM12. Volumes of interest (VOIs) were extracted from the single participant level GLM result files. The GLM contrast results for average effect of condition (language task) were displayed with a threshold of $(p<.001)$ to show activation at the regions of interest. The coordinate of interest was selected around -29 , $-91,-12$ corresponding to the most posterior section of the visual word form area (see Bouhali et al., 2014), however, the exact coordinates varied slightly between participants depending on individual anatomy and univariate-level activation. A $6 \mathrm{~mm}$ sphere was used as the radius for defining the volume of interest, correcting for the average effect of condition.

\section{Results}

We observed greater connectivity for English compared to Welsh words between the pVWFA and regions along the ventral pathway, mainly the inferior and middle temporal gyri (BA20; 
BA21) and the fusiform gyrus (BA37), as well as some regions outside this pathway bilaterally. However, the connectivity observed for Welsh compared to English words was only higher in a small portion of the left orbitofrontal area (BA11).

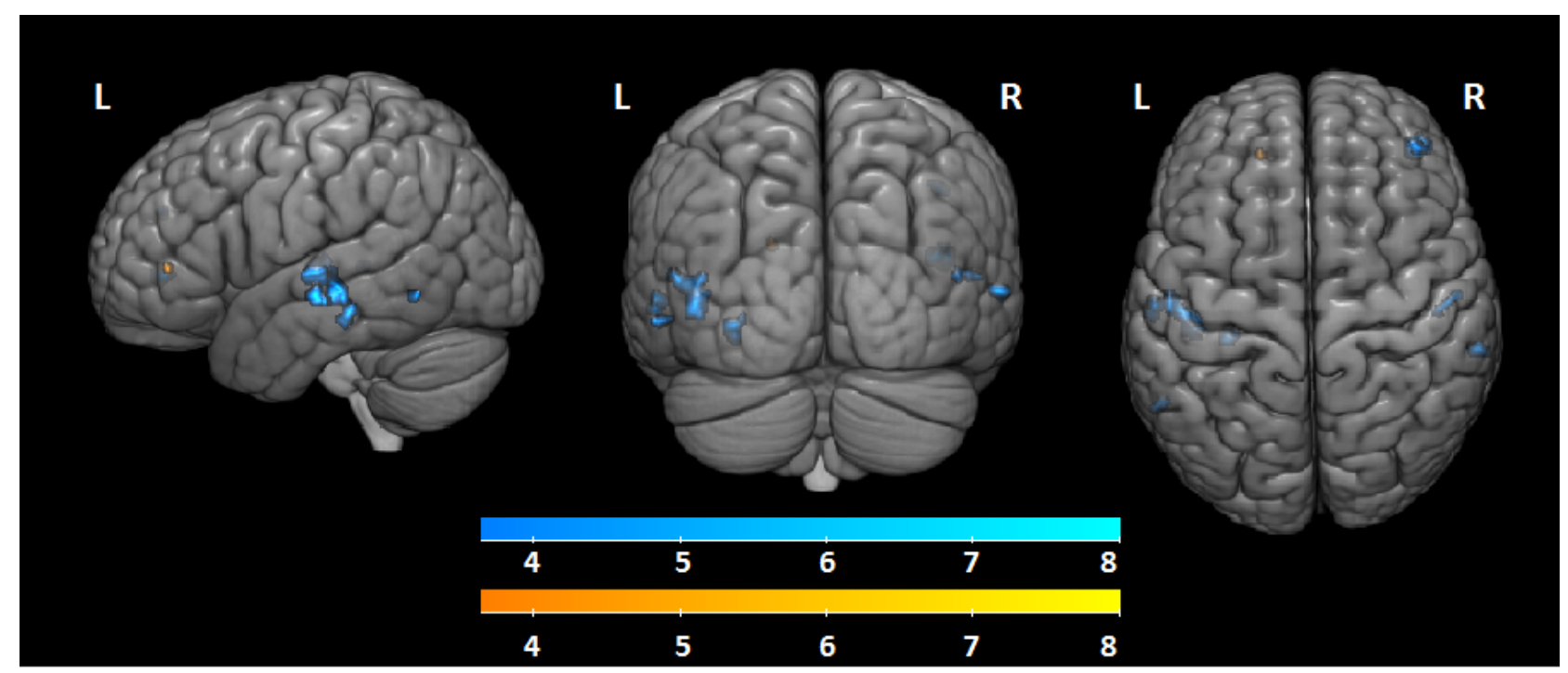

Figure 1. Contrast in functional connectivity between pVWFA and all other brain regions. 3d view. Blue: English > Welsh. Orange: Welsh > To English (t values).

\section{Discussion}

Our results suggest greater temporal coherence of the ventral lexical-semantic pathway in the deeper language of early proficient bilingual readers. However, Welsh reading for meaning did not show greater connectivity than English between pVWFA and dorsal regions. This contrasts with earlier studies and may reflect a greater convergence of reading processes across languages differing in orthographic depth in early bilinguals. However, it remains possible that stronger dorsal PPI patterns may emerge in reading tasks less explicitly reliant on processing meaning and making conscious meaning-based decisions.

\section{References}

Oliver, Myriam \& Carreiras, Manuel \& Paz-Alonso, Pedro. (2017). Functional Dynamics of Dorsal and Ventral Reading Networks in Bilinguals. Cerebral Cortex. 2\%. 10.1093/cercor/bhw310. 


\title{
Treating lexical retrieval using letter fluency in primary progressive aphasia - a single case study
}

\author{
Vânia de Aguiar ${ }^{1,2}$, Adrià Rofes ${ }^{2-4}$, Bronte Ficek ${ }^{1}$, Kimberly Webster ${ }^{1,5}$, Haley Wendt ${ }^{1}$, \\ Kyrana Tsapkini ${ }^{1,4}$ \\ ${ }^{1}$ Department of Neurology, Johns Hopkins Medicine \\ ${ }^{2}$ Department of Linguistics, University of Groningen \\ ${ }^{3}$ Global Brain Health Institute, Trinity College Dublin \\ ${ }^{4}$ Department of Cognitive Science, Johns Hopkins University \\ ${ }^{5}$ Department of Otolaryngology, Johns Hopkins Medicine
}

\section{Introduction}

In early stages of frontotemporal degeneration, individuals who develop Primary Progressive Aphasia (PPA) complain of communication difficulties, while scoring within norm in language assessments (Czarnecki et al., 2008). Given the progressive nature of the disease, it is important to intervene early (Croot, Nickels, Laurence, \& Manning, 2009). However, it is a challenge to provide therapies that are demanding enough for individuals in early stages, as most word finding therapies have been designed towards individuals with more severe deficits, typically resulting from post-stroke aphasia (e.g., Nickels, 2002). Hence, patients are often left untreated until deficits are more severe. Here, we propose using a modified letter fluency task as therapy. This task can be challenging even for healthy individuals. Furthermore, verbal fluency tasks are commonly used in cognitive assessments, as they are quick to administer, easy to score, and can be used in individuals with varying social and educational backgrounds (Da Silva et al., 2004).

Previous research has shown that transcranial Direct Current Stimulation (tDCS) to the left inferior frontal gyrus (IFG) improves written naming and spelling performance in PPA (Tsapkini et al., 2018). Additional evidence showed that tDCS over the left IFG improves letter fluency in healthy controls (Cattaneo, Pisoni, \& Papagno, 2011). In this case study, we test the efficacy of letter fluency intervention combined with left IFG anodal tDCS for a highly functioning individual with subjective complaints of word finding difficulty.

\section{Methods}

\section{Participants}

AGG is a 74 year old individual with a profile consistent with logopenic variant PPA (GornoTempini, Murray, Rankin, Weiner, \& Miller, 2004). MRI shows atrophy in parietal and frontal lobes, with left-sided asymmetry. His output is fluent, albeit with reduced speech rate. He presents word-finding difficulties and occasional phonemic paraphasias in spontaneous speech, while scoring within norm in the Boston Naming Test (25/30). His sentence comprehension is good (errors limited to comprehension of object relatives). Sentence repetition is mildly impaired, with $2 / 5$ sentences fully accurate, but $32 / 37$ words accurate. Letter fluency is within norm (FAS sum $=47,43,40$, measured 8 months, 3 months, and immediately before therapy, respectively), and so is category fluency for animals (17, 18, 14, same assessment times; Tombaugh, Kozak, \& Rees, 1999).

\section{Outcome measures}

Letter fluency in 1-minute periods was assessed for 24 letters before and after therapy. AGG was asked to produce as many words as he could think of starting with the letter provided. Three sets of eight letters were created: one to use in treatment, and two untreated sets (one to serve as control, and the other one reserved for treatment in a second therapy phase that 
is currently ongoing, where stimulation is delivered to the inferior parietal/posterior temporal lobes). The sets were matched for the number of words present in the CELEX database starting with each target letter, the average frequency of those same words, the number of vowels and consonants, and the average number of words retrieved before therapy. To study improvement in lexical retrieval in an untreated task, we additionally administered a timed object naming test, using 252 colored Snodgrass and Vanderwart's pictures (Rossion \& Pourtois, 2004).

\section{Treatment}

Intervention consisted of a letter fluency task (adapted from Tsapkini, Frangakis, Gomez, Davis, \& Hillis, 2014). AGG was given periods of 1-3 minutes (increasing across therapy sessions) to retrieve as many words as possible for each letter. An alphabet board was provided to prompt him to think of combinations of the target with other letters. For homework activities, AGG generated lists of words, while timing himself in periods of 2-5 minutes per letter, and produced the words in oral and written form. AGG occasionally consulted a dictionary during his homework. In the first 20 minutes of each session, AGG received anodal tDCS at $2 \mathrm{~mA}$, delivered using $5 \times 5 \mathrm{~cm}$ electrodes, with the anode over Broca's area and the cathode over the right cheek. Therapy was delivered across 10, daily, 45-min. Sessions.

\section{Results}

At baseline, there were no significant differences between the three sets of letters in number of words retrieved. From pre- to post-treatment measurements, there was a significant increase in number of words retrieved for three sets (altogether: $\mathrm{Z}=7.5, p<0.001$; trained: $\mathrm{Z}=0, p=0.008$; untreated1: $\mathrm{Z}=3, p=0.042$; untreated2: $\mathrm{Z}=1, p=0.021)$. A significantly larger number of words was retrieved for treated letters when compared to each untrained set (untreated1: $U=58$, $p=0.007$; untreated $2: \mathrm{U}=54, p=0.023)$. The two untreated sets did not differ significantly after treatment $(\mathrm{U}=32.5, p=1)$. In the untreated timed object naming task, AGG became significantly more accurate $(\mathrm{X} 2(1)=7.31, p<0.01)$ and slower $(\mathrm{t}(142)=-4.207, p<0.001)$ after therapy when compared to before therapy (see Figure 1, panels A to C).

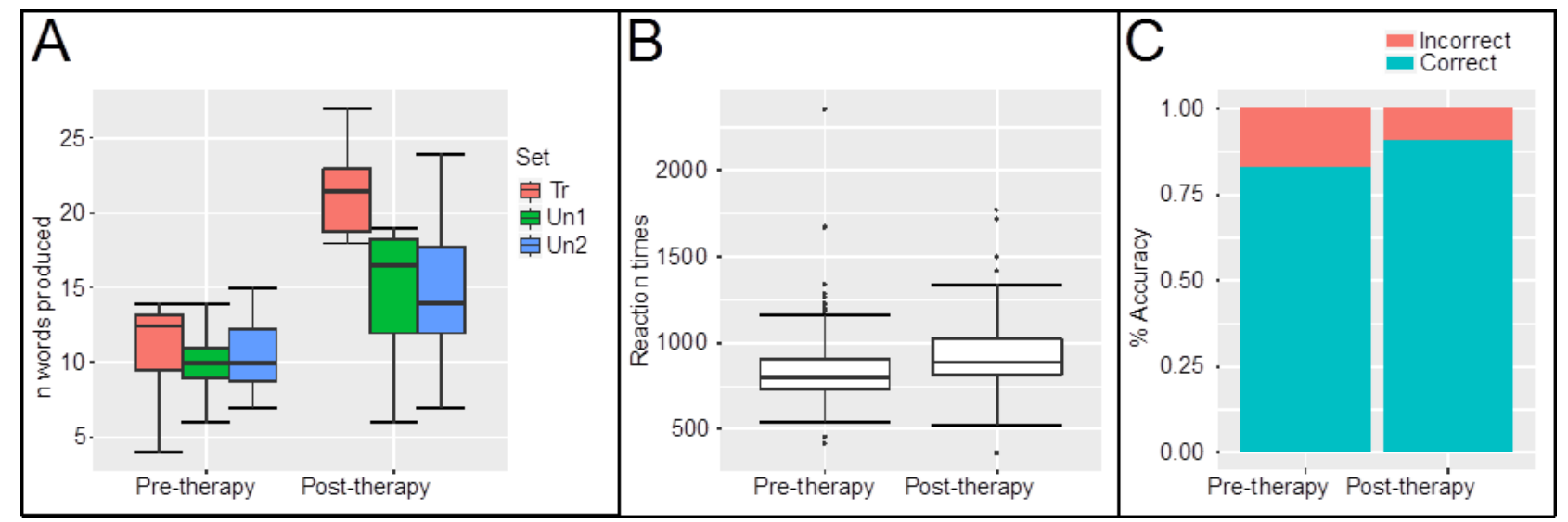

Figure 1. Therapy effects on letter fluency and naming. Panel A: mean number of words retrieved across letters in each set (Tr: Treated; Un1: Untreated1; Un2: Untreated2). Panel B: Oral reaction times in picture naming before and after therapy (including correctly named words only). Panel C: Percentage of correct responses in naming before and after therapy in picture naming.

\section{Discussion}

More research evidence is needed for interventions suitable to the progressive nature of PPA (Croot et al., 2009). Letter fluency is a challenging task even for individuals that perform within norm. Here we show that practicing letter fluency combined with tDCS in the left IFG can result in improved word retrieval both in the same task and in picture naming (where a 
tradeoff between speed and accuracy may be occurring). These results are in line with studies of healthy individuals (Cattaneo et al., 2011). The ongoing therapy phase will allow testing whether tDCS can enhance therapy effects by comparing left IFG stimulation to a control stimulation condition, the IPL, which is known to show significant atrophy in lvPPA (GornoTempini, Dronkers, et al., 2004) and to be involved in both letter and category fluency (Birn et al., 2010; Vitali et al., 2005). Furthermore, ongoing psycholinguistic analyses of words retrieved during letter fluency at each assessment time (frequency, familiarity, imageability, and concreteness of words retrieved in fluency tasks) will characterize change in terms of lexical and semantic processing (e.g., Rofes et al., 2019).

\section{References}

Cattaneo, Z., Pisoni, A., \& Papagno, C. (2011). Transcranial direct current stimulation over Broca's region improves phonemic and semantic fluency in healthy individuals. Neuroscience, 183, 64-70.

Croot, K., Nickels, L., Laurence, F., \& Manning, M. (2009). Impairment and activity/participation - directed interventions in progressive language impairment: Clinical and theoretical issues. Aphasiology, 23(2), 125-160.

Czarnecki, K., Duffy, J. R., Nehl, C. R., Cross, S. A., Molano, J. R., Jack, C. R., ... Boeve, B. F. (2008). Very Early Semantic Dementia With Progressive Temporal Lobe Atrophy: An 8-Year Longitudinal Study. Archives of Neurology, 65(12), 1659-1663. https://doi.org/10.1001/archneurol.2008.507

Da Silva, C. G., Petersson, K. M., Faísca, L., Ingvar, M., \& Reis, A. (2004). The effects of literacy and education on the quantitative and qualitative aspects of semantic verbal fluency. Journal of Clinical and Experimental Neuropsychology, 26(2), 266-277.

Gorno-Tempini, M. L., Murray, R. C., Rankin, K. P., Weiner, M. W., \& Miller, B. L. (2004). Clinical, cognitive and anatomical evolution from nonfluent progressive aphasia to corticobasal syndrome: a case report. Neurocase, 10(6), 426-436.

Nickels L. Therapy for naming disorders: Revisiting, revising, and reviewing. Aphasiology. 2002;16(10-11):935-979.

Rofes, A., de Aguiar, V., Ficek, B., Wendt, H., Webster, K., \& Tsapkini, K. (in press). The role of word properties in performance on fluency tasks in people with Primary Progressive Aphasia. Journal of Alzheimer's Disease.

Rossion, B., \& Pourtois, G. (2004). Revisiting Snodgrass and Vanderwart's object pictorial set: the role of surface detail in basic-level object recognition. Perception, 33(2), 217236.

Taylor, C., Kingma, R. M., Croot, K., \& Nickels, L. (2009). Speech pathology services for primary progressive aphasia: Exploring an emerging area of practice. Aphasiology, 23(2), 161-174. https://doi.org/10.1080/02687030801943039

Tombaugh, T. N., Kozak, J., \& Rees, L. (1999). Normative Data Stratified by Age and Education for Two Measures of Verbal Fluency: FAS and Animal Naming. Archives of Clinical Neuropsychology, 14(2), 167-177. https://doi.org/10.1016/S0887-6177(97)00095-4

Tsapkini, K., Frangakis, C., Gomez, Y., Davis, C., \& Hillis, A. E. (2014). Augmentation of spelling therapy with transcranial direct current stimulation in primary progressive aphasia: Preliminary results and challenges. Aphasiology, 28((8-9), 1112-1130.

Tsapkini, K., Webster, K. T., Ficek, B. N., Desmond, J. E., Onyike, C. U., Rapp, B., ... Hillis, A. E. (2018). Electrical brain stimulation in different variants of primary progressive aphasia: A randomized clinical trial. Alzheimer's \& Dementia: Translational Research \& Clinical Interventions, 4, 461-472. 


\title{
Who does what to whom: the role of the l-IPS in the comprehension of reversible and irreversible sentences
}

\author{
Lorenzo Vercesi $_{1,2}$, Prerana Sabnis ${ }_{1,3}$, Chiara Finocchiaro ${ }_{4}$, Luigi Cattaneo $_{5}$, Elena Tonolli ${ }_{1}$, \\ Gabriele Miceli $_{1,6}$ \\ ${ }_{1}$ CIMeC-Center for Mind/Brain Sciences, University of Trento, Italy \\ ${ }_{2}$ Department of Neurosciences, Psychology, Drug Research and Child Health \\ (NEUROFARBA), University of Florence, Italy \\ ${ }_{3}$ International Doctorate for Experimental Approaches to Language and Brain (IDEALAB), \\ University of Trento, Rovereto, Italy \\ ${ }_{4}$ DipSCo, Department of Cognitive Sciences, University of Trento, Italy \\ ${ }_{5}$ Department of Neuroscience, Biomedicine and Motion, University of Verona, Italy \\ ${ }_{6}$ Centro Linceo Interdisciplinare 'Beniamino Segre', Accademia dei Lincei, Rome, Italy
}

\section{Introduction}

In sentence comprehension different cues contribute to meaning interpretation. Among these, thematic roles are of particular interest. Given a sentence like The girl watches the tree, the listener must establish that the girl is the one who is doing the action (the agent), whereas the tree is what the action is being performed on (the patient/theme). Since thematic role assignment relies on the combination of syntactic and semantic features, its difficulty can be modulated by word order and semantic reversibility.

In SVO languages, words usually appear in canonical order in active sentences (agent-verbtheme), and in non-canonical order in passive sentences (theme-verb-agent). Therefore, interpretation is more demanding in passives. Sentence comprehension is modulated also by semantic reversibility. In irreversible sentences, only one constituent can be the agent. In the active sentence The girl watches the tree, canonical word order facilitates thematic role assignment. In the corresponding passive sentence The tree is watched by the girl, role assignment is less easy, as word order is non-canonical. However, in both cases thematic roles can be assigned based solely on semantic knowledge. In semantically reversible sentences, however, both constituents can be agent or theme. Therefore, comprehension requires syntactic processing. In active sentences (The girl kisses the boy) thematic role assignment is facilitated by canonical word order. In passive sentences (The boy is kissed by the girl), however, word order is non-canonical and semantic knowledge cannot constrain thematic role assignment. Due to non-canonical word order and semantic reversibility, these sentences must be re-analysed and thematic roles re-assigned (Chomsky, 1981; Pollard \& Sag, 1994; Bresnan, 2000; Finocchiaro et al., 2015).

Evidence from neuroimaging (Richardson et al., 2010; Walenski et al., 2019), and lesion studies (Caramazza \& Miceli, 1991; Dronkers et al., 2004; Thothathiri et al., 2012; Rogalsky et al., 2018) suggests that the left intraparietal sulcus (l-IPS) plays a critical role comprehending passive reversible sentences. In rTMS studies (Finocchiaro et al., 2015; submitted), stimulation of posterior l-IPS enhanced performance accuracy on passive (but not active) reversible sentences and pseudo-sentences.

In this study, reversible and irreversible sentences were administered. If the effect reported by Finocchiaro et al results from reversibility per se, rTMS should interfere only with reversible passives. If it results from a combination of diathesis and reversibility, both reversible and irreversible passives should be affected.

\section{Methods}

Materials

A sample of 120 active and passive, reversible and irreversible sentences was prepared. The 
same 30 verbs were used four times, once in each sentence type (reversible active, reversible passive, irreversible active, irreversible passive). Word frequency was controlled based on an Italian corpus (Bertinetto et al, 2005), and log frequency values were calculated before finalizing the list, to exclude extremely frequent or infrequent verbs/nouns.

\section{Experimental paradigm}

Twenty participants completed a sentence comprehension task that required identifying the agent or the theme of a written sentence while receiving real/sham stimulation. A sentence was presented at the center of the screen, immediately followed by two alternatives corresponding to the two constituents. Participants responded by keyboard press within a fixed temporal window of $3000 \mathrm{~ms}$ (Fig. 1). Accuracy and RTs were measured.

Repetitive TMS (rTMS) was delivered over the inferior l-IPS at 5Hz, starting at stimulus onset. Stimulation was controlled by a neuronavigation system (Softaxic). Each subject completed a sham-TMS and a real-TMS session, separated by a week. Session order was counterbalanced across participants. Stimulation site was identified by an MRI-based 3D reconstruction of the individual brain.

\section{Results}

rTMS over the l-IPS influenced RTs. There was a significant interaction of DIATHESIS (active vs passive), REVERSIBILITY (irreversible vs reversible) and STIMULATION TYPE (sham vs real) factors $(\mathrm{F}(1,23)=5.825 p=0.024)$. In particular, TMS increased RTs only on reversible passives (Bonferroni's posthoc $\mathrm{t}=-8.205, p<0.001$ ). No effect of stimulation was observed on active and passive irreversibles and active reversibles. Fig. 1 summarizes results.

\section{REVERSIBILITY: I}

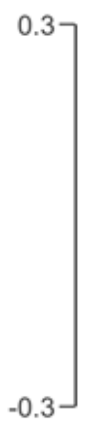

STIMULATION TYPE

O real

sham

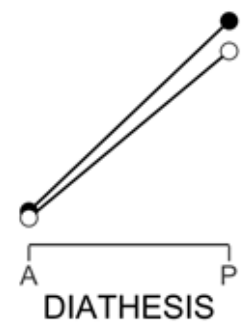

REVERSIBILITY: $\mathbf{R}$

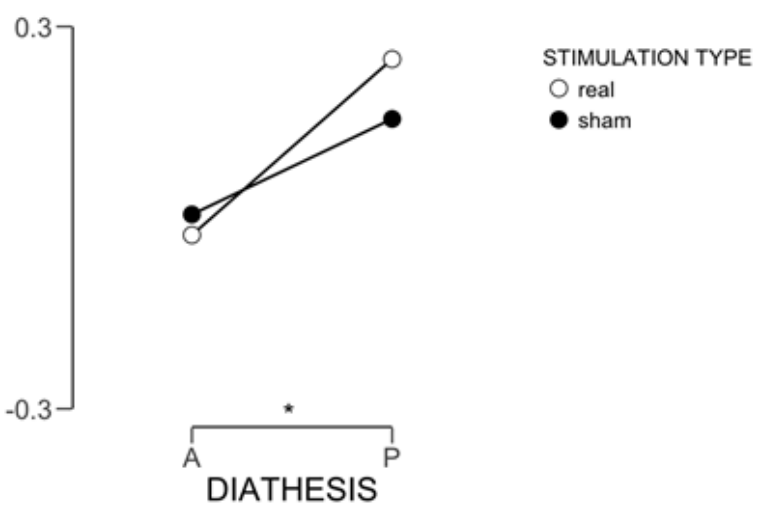

Figure 1. Summary of results. TMS increased RTs only on reversible passive sentences (RP). 


\section{Discussion}

Posterior l-IPS is involved in the comprehension of reversible passive sentences, as a consequence of the need to review the initial assignment of thematic roles in these sentences. Results shed some light on this effect. If re-analysis were constrained by reversibility per se, both active and passive reversibles should be affected by stimulation, which was not our case. On the other hand, if re-analysis were required as a consequence of diathesis per se, rTMS should affect passive sentences regardless of reversibility. Both in the present study and in previous investigations, rTMS influenced performance only on reversible passives. These results are consistent with the view that comprehending reversible passives requires re-analyzing syntactic structure to re-assign previously encoded thematic roles.

\section{References}

Bertinetto P.M., Burani C., Laudanna A., Marconi L., Ratti D., Rolando C. \& Thornton A.M. (2005) Corpus e Lessico di Frequenza dell'Italiano Scritto (CoLFIS)

Bresnan, J., 2000. Lexical-Functional Syntax. Blackwell, Oxford, UK

Caramazza A. \& Miceli G. (1991). Selective impairment of thematic role assignment in sentence processing Brain Lang 1991; 41, 402-36

Chomsky, N., 1981. Lectures on Government and Binding. Foris, Dordrecht, The Netherlands.

Dronkers N.F., Wilkins D.P., Van Valin R.D.Jr., Redfern B.B. \& Jaeger J.J. (2004). Lesion analysis of the brain areas involved in language comprehension. Cognition 92, 145-177.

Finocchiaro C., Capasso R., Cattaneo L., Zuanazzi A. \& Miceli, G. (2015). Thematic Role Assignment in the Posterior Parietal Cortex: A TMS study. Neuropsychologia 77, 223232.

Finocchiaro C., Cattaneo L. \& Miceli G. (submitted). Thematic reanalysis in the left posterior parietal sulcus: A TMS study.

Pollard, C., \& Sag, I.A., 1994. Head-Driven Phrase Structure Grammar. The University of Chicago Press, Chicago, IL, USA.

Richardson, F.M., Thomas, M.S.C. \& Price, C.J. (2010). Neuronal activation for semantically reversible sentences. J Cogn Neurosci 22, 1283-1298

Rogalsky, C., LaCroix, A.N., Chen, K.H. \& Anderson, S.W. (2018). The Neurobiology of agrammatic sentence comprehension: a lesion study. J. Cogn Neurosci 30, 234-255

Thothathiri, M., Kimberg, D.Y. \& Schwartz, M.F. (2012). The neural basis of reversible sentence comprehension: evidence from voxel-based lesion-symptom mapping in aphasia. J Cogn Neurosci 24, 212-222.

Walenski, M., Europa, E., Caplan, D. \& Thompson, C.K. (2019). Neural networks for sentence comprehension and production: An ALE-based meta-analysis of neuroimaging studies. Hum Brain Map 39, 1-30 


\title{
Using multi-word utterances more flexibly in non-fluent aphasia: Findings from a case series investigation
}

\author{
Bruns, C., Zimmerer, V., Bruce, C., Varley, R., Beeke, S. \\ Department of Language $\&$ Cognition, Division of Psychology and Language Sciences, \\ University College London
}

\section{Introduction}

Individuals with aphasia are able to use familiar multi-word utterances (high-frequency combinations such as "I went to", "I suppose" and "I don't know") for various conversational functions, for instance to hold and yield the conversational floor (e.g., Beeke, 2003; Bruns et al., 2019). While such combinations often seem fixed, many of them map onto semi-fixed frames with open slots (e.g., "[PERSON] went to [PLACE]") and thus have the potential to be used as a communicative resource in different contexts ("I went to Spain"; "you went to the station"). Fixed and semi-fixed phrases are a main feature of usage-based theories which propose that constructions (i.e., stored form-meaning pairings; Goldberg, 2003) are central to language acquisition and processing. Semi-fixed frames and more abstract constructions are thought to emerge from expressions which are repeatedly encountered in an individual's language usage (Langacker, 1991; 2008).

A common aim of aphasia research is to develop effective intervention programs that enhance the ability to access and produce multi-word utterances. As yet, however, no aphasia intervention has explicitly tested application of usage-based principles. This case series investigation piloted the impact of a novel intervention, aimed at enhancing the connected speech of individuals with non-fluent aphasia. The intervention was motivated by usage-based principles and involved loosening of open slots in semi-fixed frames such as "[REFERENT] like [THING / PROCESS / PERSON / PLACE]". We examined the intervention's outcomes and acceptability (Bruns, 2018).

\section{Methods}

\section{Participants}

We recruited five participants (P1-P5; three women, two men) with chronic, non-fluent aphasia (24 - 165 months post-onset). Participants were between 48 and 68 years old $(\mathrm{M}=59.8, \mathrm{SD}=$ 7.4) and reported English as their main language.

\section{Intervention and Outcome Measures}

Participants completed a 6 -week usage-based intervention program, designed to practice more flexible use of semi-fixed frames. The intervention was computerized and included self-managed elements. We focused on 12 corpus-derived, high-frequency phrases which mapped onto semifixed frames (e.g. "I like it": "[REFERENT] like [THING / PROCESS / PERSON / PLACE]"). All intervention stimuli were relevant to everyday talk about experiences, opinions and exchanging information. Participants practiced flexible use of constructions via errorless learning strategies, following the steps used in the SWORD program (Varley et al., 2016; Whiteside et al., 2012).

Outcome measures focused on changes in connected narrative between pre- and post-intervention and were determined by the Frequency in Language Analysis Tool (Zimmerer, Coleman, \& Wibrow, 2017). The main outcome measure was the number of three-word combinations divided by the number of words produced by a participant (combination ratio; Zimmerer et al., 2018). Moreover, we designed a study-specific Story Completion Test, adapted from Goodglass 
et al. (1972), to investigate the availability of trained constructions before and after intervention. Stability of any communicative change was explored at a 6-week follow-up assessment. The acceptability of the intervention to participants was also investigated via questionnaires.

\section{Results}

A comparison of sentence production at baseline versus post-intervention showed some evidence of enhanced connected speech for four of the five participants (increased combination ratio for P2, P3, P4 and P5 after intervention). However, only P5's increase of combination ratio exceeded baseline variation and remained stable across post-intervention probes. Story Completion Test scores revealed positive change with regard to the number of grammatically well-formed utterances produced by two participants (P1 and P2). Overall, findings were mixed with regard to baseline stability of outcome measures and post-intervention stability of communicative changes. The intervention was acceptable to all participants.

\section{Discussion}

This study yields promising findings with regard to outcomes and acceptability. The results inform participant selection for subsequent research. A next step will be to test a refined version of the intervention (e.g., more detailed tracking of dose), using a combination of frequency-based and qualitative measures to investigate change in connected speech within narrative production.

\section{References}

Beeke, S. (2003). "I suppose" as a resource for the construction of turns at talk in agrammatic aphasia. Clinical Linguistics \&6 Phonetics, 17(4-5), 291-298. https://doi.org/10.1080/0269920031000080055

Bruns, C. (2018). A usage-based approach to language processing and intervention in aphasia. Doctoral Thesis. University College London.

Bruns, C., Varley, R., Zimmerer, V.C., Carragher, M., Brekelmans, G. \& Beeke, S. (2019). "I don't know": a usage-based approach to familiar collocations in non-fluent aphasia. Aphasiology, 33(2), 140-162. https://doi.org/10.1080/02687038.2018.1535692

Goldberg, A. E. (2003). Constructions: a new theoretical approach to language. Trends in Cognitive Sciences, 7(5), 219-224. https://doi.org/10.1016/S1364-6613(03)00080-9

Goodglass, H., Gleason, J. B., Ackerman Bernholtz, N., \& Hyde, M. R. (1972). Some Linguistic Structures in the Speech of a Broca's Aphasic. Cortex, 8(2), 191-212. https://doi.org/10.1016/S0010-9452(72)80018-2

Langacker, R. W. (1991). Foundations of cognitive grammar. Volume II. Descriptive Application. Stanford, CA: Stanford University Press.

Langacker, R. W. (2008). Cognitive Grammar: A basic introduction. New York: Oxford University Press.

Varley, R., Cowell, P. E., Dyson, L., Inglis, L., Roper, A., \& Whiteside, S. P. (2016). SelfAdministered Computer Therapy for Apraxia of Speech: Two-Period Randomized Control Trial with Crossover. Stroke, 47(3), 822-828. https://doi.org/10.1161/STROKEAHA.115.011939

Whiteside, S. P., Inglis, A. L., Dyson, L., Roper, A., Harbottle, A., Ryder, J., ... Varley, R. A. (2012). Error reduction therapy in reducing struggle and grope behaviours in apraxia of speech. Neuropsychological Rehabilitation, 22(2), 267-294.

https://doi.org/10.1080/09602011.2011.639614

Zimmerer, V.C., Coleman, M., \& Wibrow, M. (2017). Frequency in Language Analysis Tool.

Zimmerer, V.C., Newman, L., Thomson, R., Coleman, M., \& Varley, R.A. (2018). Automated analysis of language production in aphasia and right-hemisphere damage: frequency and collocation strength. Aphasiology, 32(11), 1267-1283. 


\title{
Prosody perception by Slovene speaking individuals diagnosed with Parkinson's Disease
}

\author{
Maja Blesić ${ }^{1}$, Dejan Georgiev ${ }^{2}$, Christina Manouilidou ${ }^{2,3}$ \\ ${ }^{1}$ MEi:CogSci, Faculty of Education, University of Ljubljana, Slovenia \\ ${ }^{2}$ Department of Neurology, University Medical Centre, Ljubljana, Slovenia \\ ${ }^{3}$ Department of Comparative and General Linguistics, University of Ljubljana, Slovenia
}

\section{Introduction}

Parkinson's disease (PD), a chronic neurodegenerative disorder, has recently been investigated for its non-motor symptoms such as linguistic processing and non-verbal communication. Several studies (Ariatti, Benuzzi \& Nichelli, 2008; Dara, Monetta \& Pell, 2008; Pell, 1996; Pell \& Leonard, 2003; Ventura et al., 2012) have found perceptive communication and comprehension of prosodic meanings to be impaired in individuals affected by PD. Prosodic information, encoded in acoustic features such as intensity, vocal pitch, and rate of utterance, is essential for the distinction between sentence types (e.g. questions, statements) and for the recognition of the emotional tone of the utterances (e.g. happy, sad). Previous studies have found PD patients to have difficulties in: recognizing emotions from speech (Ariatti, Benuzzi \& Nichelli, 2008; Pell \& Leonard, 2003; Ventura et al., 2012, Yip et al., 2003), recognizing speaker's confidence level (Monetta, Cheang \& Pell, 2008) and recognizing linguistic modality of an utterance (Ariatti, Benuzzi \& Nichelli, 2008; Pell 1996). Studies focusing on emotional prosody recognition in PD have reported an impaired recognition of prosody for specific emotions (Dara, Monetta \& Pell, 2008; Ventura et al., 2012; Yip et al., 2003). Until now, the perception of emotional and linguistic prosody has been investigated in Germanic languages (e.g. English; Pell, 1996) and Romance languages (e.g. Italian; Ariatti, Benuzzi \& Nichelli, 2008). The current study investigated the perceptive ability of prosodic cues in Slovene-speaking individuals affected by PD, therefore bringing for the first time evidence from a Slavic language. A prosody recognition paradigm consisting of a combination of a discrimination and an identification task was administered to the participants. Along the lines of Pell (1996), we expect PD patients to be impaired in categorizing emotional and linguistic prosodic meanings but no impairment is expected in their capacity to discriminate between two different types of prosody.

\section{Methods}

Participants

Ten individuals, native speakers of Slovene, diagnosed with PD are planned to be included in the experimental group, and their results will be compared to ten healthy control (HC) individuals matched for age, gender, and years of education. Here we present current results from $3 \mathrm{PD}$ (mean age $=77.73 \pm 6.43)$ compared to $1 \mathrm{HC}($ age $=70)$. The neuropsychological assessment of participants included the administration of the Mini Mental State Examination (MMSE) and the Montreal Cognitive Assessment (MoCA). Exclusion criteria involved: depression, hearing impairments and dementia.

\section{Materials}

A new audio dataset of stimuli was built for the purpose of this study and validated by 10 raters. The stimuli consist of audio recordings of pseudo-utterances uttered by an actress. The pseudo-sentences obeyed phonotactic and morphosyntactic rules of Slovene (e.g. "Prohast katoh groji zdrog"). Included in the narrow stimuli selection were only samples that scored high on the recognition test for raters (at least 70\%). For the linguistic prosody condition we used stimuli that were uttered as a questions and as a statement (20 utterances: 10 statements, 10 questions). For the emotional prosody condition we used stimuli uttered in 6 distinct emo- 
tional tones: anger, sadness, disgust, fear, happiness and pleasant surprise (42 utterances: 7 utterances x 6 emotional categories).

Tasks

For both experimental conditions (linguistic and emotional) we administered an off-line identification task followed by the corresponding off-line discrimination task. In the identification task single stimuli were presented in each trial (linguistic prosody condition: 20 trials; emotional prosody condition: 42 trials) and participants were asked to recognize stimuli belonging to distinct linguistic or emotional categories. In the discrimination task, participants were presented with pairs of stimuli in each trial (linguistic prosody condition: 16 trials, emotional prosody condition: 20 trials) and were asked to judge whether they are the same or different in regard to prosody. Participants listened to the stimuli through headphones connected to a touch screen laptop on which they would give their responses.

\section{Results}

All the results are reported in terms of mean percentage of correct responses. In the linguistic prosody condition, we observed a lower performance for PD in both identification $(82 \%)$ and discrimination (71\%) tasks compared to the performance of $\mathrm{HC}$ in identification (100\%) and discrimination (95\%). tasks. In the linguistic identification task PD performed better in recognizing statements $(87 \%)$ than questions $(73 \%)$. In the linguistic discrimination task $\mathrm{PD}$ recognized the same prosody pairs of utterances with the same accuracy rate $(85 \%)$ as different prosody pairs of utterances (85\%). In the emotional prosody condition we observed a lower performance for PD (32\%) in the identification task compared to HC (43\%), while PD's performance $(78 \%)$ was comparable to $\mathrm{HC}(85 \%)$ in the discrimination task. The two groups differed in recognizing specific emotions within the emotional identification task. PD was less accurate in recognizing anger (57\%), sadness $(33 \%)$, disgust $(19 \%)$ and fear $(29 \%)$ compared to $\mathrm{HC}$ : anger $(71 \%)$, sadness $(71 \%)$, disgust $(57 \%)$, and fear $(57 \%)$.

\section{Discussion}

Our preliminary results suggest an impaired emotional prosody perception similar to English (Pell, 1996; Pell \& Leonard, 2003) and Italian (Ariatti, Benuzzi \& Nichelli, 2008). In tasks assessing linguistic prosody, our PD performed worse than HC not just in identifying linguistic prosody but also in discriminating it. This finding is in contrast with Pell (1996) reporting linguistic prosody impairments only in identification tasks. In line with previous research (Dara, Monetta \& Pell, 2008; Pell and Leonard 2005; Ventura et al., 2012; Yip et al., 2003) we also observed a reduced recognition for negative emotions. This study is the first attempt to investigate how Slovene speaking individuals affected by PD perceive prosodic cues that convey emotional and linguistic information on sentence level. However, further data collection and analysis are needed for more accurate conclusions.

\section{References}

Ariatti, A., Benuzzi, F., \& Nichelli, P. (2008). Recognition of emotions from visual and prosodic cues in Parkinson's disease. Neurological Sciences, 29(4), 219.

Dara, C., Monetta, L., \& Pell, M. D. (2008). Vocal emotion processing in Parkinson's disease: reduced sensitivity to negative emotions. Brain research, 1188, 100-111.

Monetta, L., Cheang, H. S., \& Pell, M. D. (2008). Understanding speaker attitudes from prosody by adults with Parkinson's disease. Journal of neuropsychology, 2(2), 415-430.

Pell, M. D. (1996). On the receptive prosodic loss in Parkinson's disease. Cortex, 32(4), 693-704.

Pell, M. D., \& Leonard, C. L. (2003). Processing emotional tone from speech in Parkinson's 
disease: a role for the basal ganglia. Cognitive, Affective, $\&$ Behavioral Neuroscience, 3(4), 275-288.

Ventura, M. I., Baynes, K., Sigvardt, K. A., Unruh, A. M., Acklin, S. S., Kirsch, H. E., \& Disbrow, E. A. (2012). Hemispheric asymmetries and prosodic emotion recognition deficits in Parkinson's disease. Neuropsychologia, 50(8), 1936-1945.

Yip, J. T., Lee, T. M., Ho, S. L., Tsang, K. L., \& Li, L. S. (2003). Emotion recognition in patients with idiopathic Parkinson's disease. Movement Disorders, 18(10), 1115-1122. 


\title{
Repeated item exposure effects in a verbal short-term memory treatment
}

\author{
Julie Schlesinger, Jessica Obermeyer, Nadine Martin \\ Eleanor M. Saffran Center for Cognitive Neuroscience, Department of Communication \\ Sciences and Disorders, Temple University
}

\section{Introduction}

Repetition priming is commonly incorporated into direct treatments of word retrieval disorders in aphasia with the expectation that repeating a word which is difficult to produce independently (e.g., in picture naming) elevates the activation of that word's input and output representations for a brief period, and this elevated activation facilitates retrieval of that word in a subsequent naming task (Weigl, 1961). Recent models of word processing have focused on the temporal component of word retrieval and propose that repetition and naming impairments arise from an imbalance of connection strength and/or short-term maintenance of activation that supports word processing (e.g., Dell et al., 1997). This model motivated our use of a treatment approach for word retrieval that adds a short-term memory component to repetition priming (repetition after a response delay) to promote strength and duration of the activation of a word's representations. Although this approach has yielded robust effects in some cases (e.g., Kalinyak-Fliszar et al., 2011), the delayed repetition/recall paradigm creates a confound between potential sources of that improvement if the same items are used in every training trial. In that case, improvement can be attributed to repeated exposure to a set of items being trained or to improved strength/maintenance of word activation in response to the repetition + response delay treatment. We addressed this question in a recent study (Martin, Rosenberg, Minkina, Schlesinger, Obermeyer, under review) that used delayed repetition/recall of words and word-sequences paradigm but reduced instances of repeated exposure of items in all phases of treatment (i.e., unique items as much as possible in baseline, training, within training probes and maintenance probes). Overall results of that study showed low effect sizes on the treatment task, but modest improvements for some of the participants on verbal span, naming and discourse tasks. In this study, we examine the question of repeated item exposure effects in the delayed repetition/recall task by using a mix of training items, some repeatedly exposed throughout training and some not.

The specific aims of this project were to determine whether repetition-based verbal short-term memory (STM) treatment for aphasia (i.e., delayed recall of multiple words) leads to:

1) Improvement in repeated items

2) Improvement on novel items

\section{Methods}

\section{Participants}

Five individuals with aphasia due to left hemisphere stroke who were at least 1 year post onset participated in this study.

\section{Treatment design}

A single subject treatment design was employed which included baseline assessment, treatment, post-treatment assessment and a three-month post-treatment follow-up. Prior to treatment enrollment, participants completed a screening to determine eligibility which consisted of immediate and 5 -second delay recall of concrete word pairs. Accuracy scores $>80 \%$ or $<10 \%$ indicated that the participant was ineligible to participate. All eligible participants were enrolled in a treatment condition of repeating concrete noun word pairs after a 5 -second delay. 


\section{Probes and control tasks}

Participants received a minimum of 3 baseline probes of 20 word strings over one-two weeks. Probes included listening to word pairs, waiting 5 -seconds, and then repeating the word pair. If performance was not stable ( $>15 \%$ among baselines), an additional baseline was administered. Probe tasks included 15 repeated sets of concrete nouns and 5 novel pairs of concrete nouns for a total of 20 words pairs. Probes were administered at the beginning of each session and followed by two control tasks. Controls included a linguistic task (nonword reading) and a nonlinguistic task (5-point test). Three post-treatment probes were administered 1 week following treatment completion and two 3-month follow-up probes were also administered to evaluate maintenance.

\section{Treatment}

Participants completed 9 treatment sessions over 3 weeks (sessions 3 times a week). Treatment included repetition of 40 word pairs at a 5 -second delay in which participants would hear a pair of words, wait 5 seconds, and then repeat the words back after a beep. Timing and beep were programmed into E-prime to ensure accurate timing and administration. Treatment stimuli consisted of balanced 2- and 3-syllable concrete words in pairs all of which were novel.

\section{Statistical analysis}

To quantify treatment effectiveness, standard mean differences were calculated between baseline and post-treatment probes and baseline and 3-month follow-up with the following formula: Mean $_{\text {posttreatment-Mean }}$ baseline $/ \mathrm{SD}_{\text {baseline }}$. Effect sizes $>2.6$ were considered small, those $>3.9$ were considered medium, and those $>5.8$ were considered large (Beeson \& Robey, 2006). Individual effect sizes were calculated for repeated and novel probe items and consisted of four measures: pairs produced correctly in serial order (ISO) and in any order (IAO), as well as the proportion of total correct items produced ISO and IAO.

\section{Results}

Question 1. Determine whether a repetition-based verbal STM treatment for aphasia leads to improvements in repeated items.

Two participants demonstrated small-medium effects on production of repeated word pairs ISO and IAO. For three of five participants, there was an increase in proportion of individual repeated items produced correctly in ISO or IAO, as evidenced by small to medium effects (Table 1).

Question 2. Determine whether a repetition-based verbal STM treatment for aphasia leads to improvements in novel items.

One of the five participants improved on the proportion of novel individual items repeated ISO (small effect).

\section{Control task.}

One participant improved on the nonword reading control task, showing a medium effect size (5.52). The remaining participants demonstrated stable or declining performance on both control tasks.

\section{Discussion}

Previous research from our lab has reported robust acquisition of treated items when training and probe items were repeated (Kalinyak-Fliszar et al., 2011) but poorer acquisition when unique items comprised each probe trial (Martin et al., under review). Here we included both types of probe trials (same words and unique words across trials) in a delayed repetition/recall 
Table 1. Participant effect sizes. Note. Pre=pre-treatment; post=post-treatment; maint=3-month post-treatment follow-up; ${ }^{*}=$ small effect size; ${ }^{* *}=$ medium effect size; $\mathrm{UtC}=$ unable to calculate due to no variance at baseline

\begin{tabular}{|c|c|c|c|c|}
\hline $\begin{array}{l}\text { Participant ID and } \\
\text { testing period }\end{array}$ & Pairs ISO & Pairs IAO & Number correct ISO & Number correct IAO \\
\hline \multicolumn{5}{|l|}{$\mathrm{CN}$-effect sizes } \\
\hline repeated pre-post & 1.73 & 1.73 & $4.04 * *$ & UtC \\
\hline repeated pre-maint & 0.289 & -0.29 & 2.31 & $\mathrm{UtC}$ \\
\hline novel pre-post & 1.15 & 1.15 & $2.89^{*}$ & 0.87 \\
\hline novel pre-maint & 1.44 & 1.44 & 2.31 & 0.58 \\
\hline \multicolumn{5}{|l|}{ KC-effect sizes } \\
\hline repeated pre-post & 2.31 & 2.31 & $3.46^{*}$ & 1.85 \\
\hline repeated pre-maint & $3.32^{*}$ & $3.32^{*}$ & $4.91 * *$ & $2.71^{*}$ \\
\hline novel pre-post & -1.44 & -1.15 & -1.44 & -0.86 \\
\hline novel pre-maint & -1.15 & -1.15 & -1.15 & -1.15 \\
\hline \multicolumn{5}{|l|}{ KG-effect sizes } \\
\hline repeated pre-post & 0 & -0.58 & 1.15 & 0.58 \\
\hline repeated pre-maint & -0.5 & -1.44 & -1.59 & -2.31 \\
\hline novel pre-post & UtC & $\mathrm{UtC}$ & 1.15 & 0.65 \\
\hline novel pre-maint & UtC & UtC & 0.58 & 0.22 \\
\hline \multicolumn{5}{|l|}{ KK-effect sizes } \\
\hline repeated pre-post & 1.68 & 1.68 & 1.56 & 1.7 \\
\hline repeated pre-maint & 0.86 & 1.24 & 1.07 & 1.27 \\
\hline novel pre-post & 1.15 & 0.72 & 1.64 & -0.63 \\
\hline novel pre-maint & 0.867 & 0.5 & 0.55 & 0.21 \\
\hline \multicolumn{5}{|l|}{ XH-effect sizes } \\
\hline repeated pre-post & $3.67^{*}$ & UtC & $2.89 *$ & 1.73 \\
\hline repeated pre-maint & $5.5 * *$ & UtC & $4.62 * *$ & $3.61^{*}$ \\
\hline novel pre-post & -0.58 & 0 & -1.33 & -0.67 \\
\hline novel pre-maint & 0.29 & 0.29 & 0 & 0 \\
\hline
\end{tabular}


task, with all novel items used in treatment, and hypothesized that effects sizes would be greater for the probes with the same items repeated on each trial. This hypothesis was confirmed in three of the five cases for both acquisition and follow-up probes. These findings suggest a need for further investigation of the relative contributions of repeated item exposure and verbal STM on effects of repetition treatments with and without a delayed response.

\section{References}

Beeson, P. M., \& Robey, R. R. (2006). Evaluating single-subject treatment research: Lessons learned from the aphasia literature. Neuropsychology review, 16(4), 161-169.

Dell, G.S., Schwartz, M.F., Martin, N., Saffran, E.M. \& Gagnon, D.A. (1997). Lexical access in aphasic and non-aphasic speakers. Psychological Review, 104 (4), 801-838.

Kalinyak-Fliszar, M., Kohen, F. P., \& Martin, N. (2011). Remediation of language processing in aphasia: Improving activation and maintenance of linguistic representations in (verbal) short-term memory. Aphasiology, 25(10), 1095-1131.

Martin, N., Rosenberg, S., Schlesinger, J., Minkina, I., \& Obermeyer, J. (under review). Treatment of verbal short-term memory abilities to improve language function in aphasia.

Weigl, E. (1961). The phenomenon of de-blocking in aphasia. Zeitschrift für Phonetik: Sprachwissenschraft und Kommunikationforschung, 14, 337-364. 


\title{
Do you prefer playing "with fire" or "with the flame"? Idiom comprehension in individuals with mild aphasic symptoms
}

\author{
Matthias J.H. Sandmann, Sabine Weiss, Horst M. Müller \\ Experimental Neurolinguistics Group $\&$ Cluster of Excellence Cognitive Interactive Technology \\ (CITEC), Bielefeld University, Bielefeld, Germany
}

\section{Introduction}

Figurative language is a complex phenomenon and part of everyday language. Idioms as a form of figurative language are familiar, fixed phrases that do not express the meaning their constituents provide (e.g., Gibbs, 1999). Processing of idioms might be impaired in patients after a cerebrovascular accident, and might range from small disorders on a literal level up to complex impairments on a communicative-pragmatic level (e.g., Papagno et al., 2004; ReyesAguilar et al., 2018). Some of these patients (particularly when they are suffering from mild aphasia only) may experience these disorders just as subliminal language limitations, which nevertheless might inhibit their ability to work or restrict their quality of life.

The aim of this study was to gain insight into how individuals with mild aphasia deal with the comprehension of different idioms in the presence of semantical or phonological distractors. In an ordinary conversation, idioms may be accidentally modified by a speaker either by so-called slip of the tongue phenomena or by semantic word substitutions (e.g., Harley, 2006). The ability to recognize these modifications might be hampered in language impaired individuals.

Our study examined the processing of plausible and frequent figurative German idioms with altered parts of their internal valence in a decision time task (reaction time). We hypothesized that participants with aphasia would react slower and identify fewer modifications correctly with increasing age due to the tendency of being more easily distracted by less relevant information. We also expected improved decision times and accuracy when idioms were primed by phonematically altered idioms due to less processing effort according to the logogen model (Morton, 1980).

\section{Methods}

\section{Participants}

Thirty German native speakers were tested and re-diagnosed with the Aachen Aphasia Test (AAT) (Huber et al., 1983) while being in the subacute or chronic stage after stroke $(\mathrm{M}=68.4$ months post onset, $\mathrm{SD}=61.9$ ). Nineteen participants (average age: 66.3 years, $\mathrm{SD}=12.3$, eight females) met the criteria of a mild aphasia (Jaecks, 2015) and were recruited for the experiment.

\section{Design \& Procedure}

A decision time experiment was conducted in which each participant was asked to identify genuine German idioms. Each trial began with a fixation cross followed by a prime of $4 \mathrm{~s}$. The prime consisted of either a phonematically modified idiom (e.g., "alte Grüße bekommen" / "to get old greetings") or a semantically modified version (e.g., "kalte Beine bekommen" / "to get cold legs"). After the prime, two different items were shown: the target idiom (e.g., "kalte Füße bekommen" / "to get cold feet") and a linguistically modified distractor idiom. In total there were 56 of these triads. Additionally, 88 filler triads were used to distract from the actual goal of the experiment. Participants were shown all triads in random sequences.

All linguistic modifications were subject to strict psycholinguistic criteria (e.g., matched internal and external valences, syntactical, and syllable structures), previously tested successfully 
in a healthy population (Sandmann et al., 2015).

\section{Statistical Analysis}

Two 2-factor mixed-design ANOVAs were used to compare decision time and accuracy (in \%) of identifying genuine idioms. Tukey's test was used for testing statistical significance concerning possible differences between variables. We considered differences dependent on age and priming idioms. For the mixed-design analyses, 10 elderly (average age: 76.8, SD = 8.2) and 9 middle-aged (average age: $56.9, \mathrm{SD}=6.0$ ) participants were divided into two age groups according to the mean retirement age in Germany of 67 years.

\section{Results}

1) Middle-aged participants made their choices after an average of $3152 \mathrm{~ms}(\mathrm{SD}=556 \mathrm{~ms})$, whereas elderly participants needed $3641 \mathrm{~ms}(\mathrm{SD}=964 \mathrm{~ms})(F(1,104)=25.1, p<.001)$.

2) Considering the idiom modifications, all participants reacted significantly faster to the target idiom primed by the semantically modified idioms $(\mathrm{M}=3180 \mathrm{~ms}, \mathrm{SD}=617 \mathrm{~ms})$ than by the phonematically modified idioms $(\mathrm{M}=3613 \mathrm{~ms}, \mathrm{SD}=941 \mathrm{~ms})(F(1,104)=16.7, p<.001)$.

3) Post-hoc tests showed that middle-aged participants responded on average $662 \mathrm{~ms}$ faster than the elderly participants to the target idioms primed by the phonematic prime $(t(208)=-4.5$, $\left.p_{\text {Tukey }}<.001\right)$. Regarding the semantic prime, the middle-aged responded on average $367 \mathrm{~ms}$ faster to the genuine target idiom than the elderly $\left(t(208)=-2.5, p_{\text {Tukey }}=.06\right)$, see figure 1 .

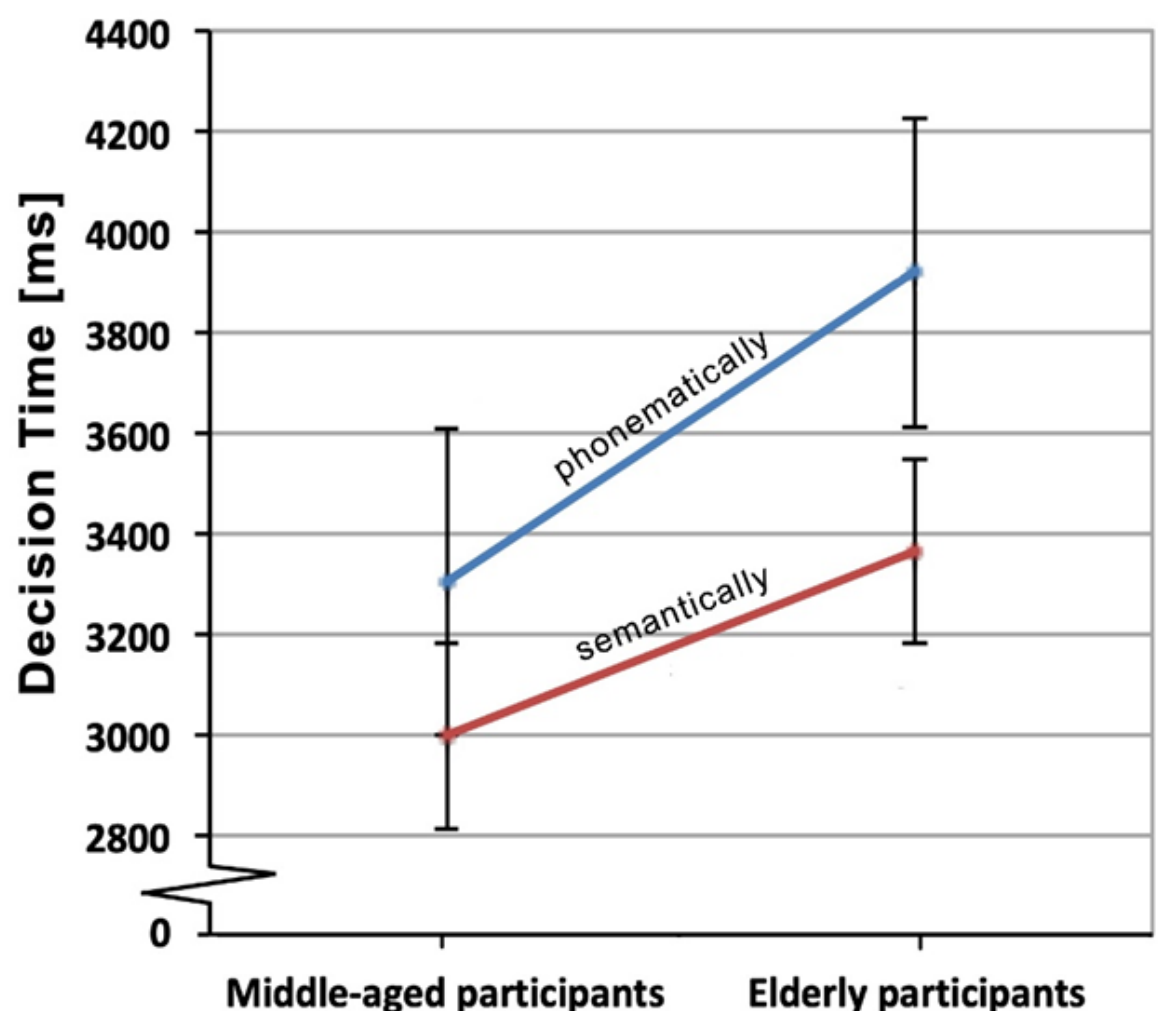

Figure 1. Differences between participants regarding the phonematically and semantically modified idioms.

With respect to response accuracy, both age groups showed comparable results: After the phonematic prime, middle-aged participants gave $94.5 \%$ (SD = 9.8 \%) accurate answers, the elderly: $95.2 \%(\mathrm{SD}=11.1 \%)$. After the semantic prime, the middle-aged group was correct 
in $95.3 \%(\mathrm{SD}=11.4 \%)$, the elderly in $97.2 \%(\mathrm{SD}=7.5 \%)$ of their decisions.

\section{Discussion}

Concerning decision time, significant differences were found when analyzing both parameters age and prime. Regardless of the age groups, all participants responded significantly faster after being presented a semantic prime than a phonematic one. This difference might be due to a decrease of the efficiency of the visual and auditory systems in aging (in this case important for grapheme-phoneme conversation after the phonematically modified prime) (Wingfield et al., 2006). As expected, middle-aged participants with aphasia responded significantly faster than elderly participants independent of the type of prime. In contrast, the mean accuracy of all participants was generally high (> 94 These findings suggest that elderly individuals do not automatically lose the ability of idiom processing following mild aphasia. They may just need more time for responding correctly due to normal aging that inhibits processing time. This explanation is in line with our findings that their accuracy rate is as good as that of the middle-aged participants. In conclusion, individuals with mild aphasic symptoms do not need to be uncertain in dealing with idioms according to this study. Therefore, speech therapists and others have to continue being patient even with individuals showing only mild aphasic symptoms.

\section{References}

Gibbs, R.W. (1999). Figurative language. In: Wilson, R., Keil, F. (eds.), The MIT encyclopedia of the cognitive science. (pp. 314-315). Cambridge, MA: MIT Press.

Harley, T. (2006). Speech errors: Psycholinguistic approach. The Encyclopedia of Language and Linguistics (2nd Ed, Vol. 11: pp. 739-744), Oxford: Elsevier.

Huber, W., Poeck, K., Weniger, D., \& Willmes, K. (1983). Aachener Aphasie Test (AAT). Göttingen: Verlag für Psychologie.

Jaecks, P. (2015). Restaphasie Forum Logopädie. Stuttgart: Thieme.

Morton, J. (1980). The logogen model and orthographic structure. In: Firth, U. (ed.) Cognitive Processes in Spelling (pp. 117-133). London: Academic Press.

Papagno, C., Tabossi, P., Colombo, M.R., \& Zampetti, P. (2004). Idiom comprehension in aphasic patients, Brain and Language, 89, 226-234.

Reyes-Aguilar, A., Valles Capetillo, E., \& Giordano, M. (2018). A quantitative meta-analysis of neuroimaging studies of pragmatic language comprehension: in search of a universal neural substrate, Neuroscience, 395, 60-88.

Sandmann, M.J.H., Weiss, S., \& Müller, H.M. (2015). Bitte »auf Schmerz und Viren prüfen«! Eine Studie zur Erkennung von figurativ-ambigen idiomatischen Wendungen im Kontext sprachlicher Distraktoren. German Association for Applied Linguistics (GAL e.V.), Frankfurt (Oder).

Wingfield, A., McCoy, S.L., Peelle, J.E., Tun, P.A., \& Cox, L.C. (2006). Effects of Adult Aging and Hearing Loss on Comprehension of Rapid Speech Varying in Syntactic Complexity, Journal of the American Academy of Audiology, 17, 487-497. 


\title{
Morpheme processing in the ventral temporal lobe: An MEG study of Japanese verbs
}

\author{
Shinri Ohta ${ }^{1,2}$, Yohei Oseki ${ }^{2,3,4}$, Alec Marantz ${ }^{2,5,6}$ \\ ${ }^{1}$ Department of Linguistics, Faculty of Humanities, Kyushu University, Fukuoka, Japan \\ ${ }^{2}$ Department of Linguistics, New York University, New York, United States \\ ${ }^{3}$ Faculty of Science and Engineering, Waseda University, Tokyo, Japan \\ ${ }^{4}$ Center for Advanced Intelligence Project, RIKEN, Tokyo, Japan \\ ${ }^{5}$ Department of Psychology New York University, New York, United States \\ ${ }^{6}$ NYUAD Institute, New York University Abu Dhabi, Abu Dhabi, United Arab Emirates
}

\section{Introduction}

Previous magnetoencephalography (MEG) studies reported that morphologically complex words are decomposed into morphemes around $170 \mathrm{~ms}$ after the onset of visual stimuli (M170) in the left fusiform gyrus and inferior temporal gyrus (FG/ITG) (Solomyak and Marantz 2009, 2010). Moreover, another MEG study found that transition probability between morphemes (morphTP) was negatively correlated with the amplitude of the M170 (Lewis et al. 2011). As these studies targeted English, in which morphological boundaries are a subset of letter boundaries, it is difficult to examine whether the M170 is modulated by morphTP or TP between letters (letterTP).

To disentangle morphological processing and letter recognition, we targeted the Japanese language, which uses logographic kanji and moraic kana writing system. For example, in Japanese verbs 染まる (som-ar-u, dye), kanji (染) represents the (part of) verbal root (e.g., som-), while kana (まる) represents voice (-ar) and tense $(-\mathrm{u})$ morphemes, leading to a mismatch between morpheme boundaries and letter boundaries (Figure 1A). Thus, Japanese is an ideal language to test morphological processing and letter recognition in the brain.

Following the previous MEG studies, we compared the effects of morphTP (i.e., TP between verbal root and suffixes) and letterTP (i.e., TP between kanji and kana) on the left FG/ITG activation in the present MEG experiment.

\section{Methods}

Participants

We recruited 22 native speakers of Japanese (nine males, $35.5 \pm 7.3$ (mean \pm SD) yrs.). All participants in the experiments were healthy and right-handed (laterality quotients: 40-100), according to the Edinburgh inventory (Oldfield 1971)

\section{Stimuli and Task}

We used 112 Japanese verbs for each of intransitive verbs, transitive verbs, intransitive-causative verbs, and transitive-causative verbs, as well as the same number of nonwords (total 896 stimuli). The participants performed a visual lexical decision task in the MEG scanner.

\section{$M E G$ data acquisition and $M E G$ data analyses}

We used a 157-channel axial gradiometer MEG system (Kanazawa Institute of Technology, Japan). For the MEG analyses, we used spatiotemporal cluster permutation tests as implemented in MEG-Python (Gramfort et al. 2013) and Eelbrain packages

(https://pythonhosted.org/eelbrain/) (see Gwilliams et al. 2016 for the details). As our primary target was the M170, the region of interest was anatomically defined as the left FG/ITG and the analysis time window was restricted to 50-250 ms after word onset. 


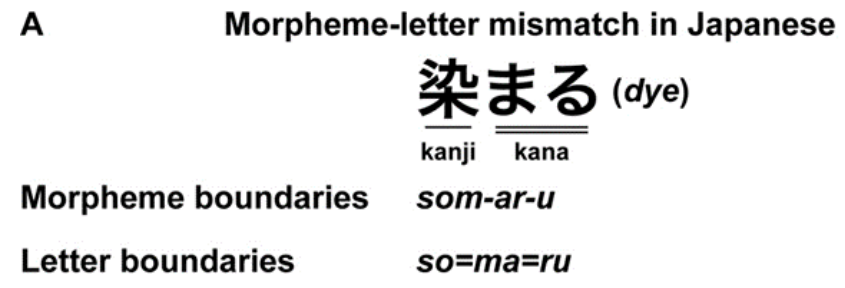

B

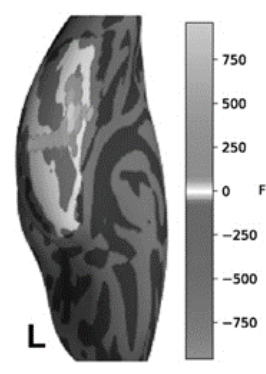

C

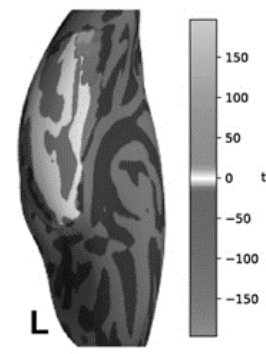

Causative > Noncausative

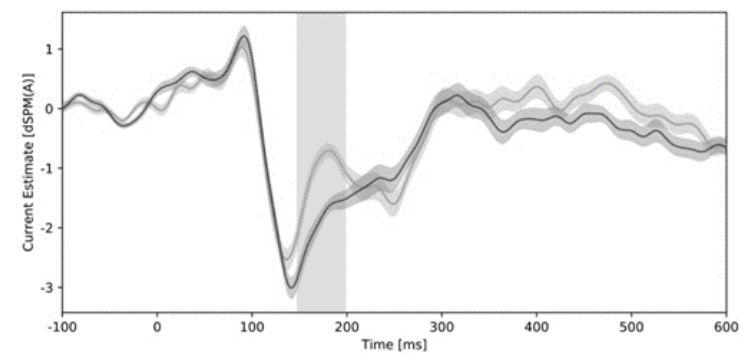

Morphological transition probability

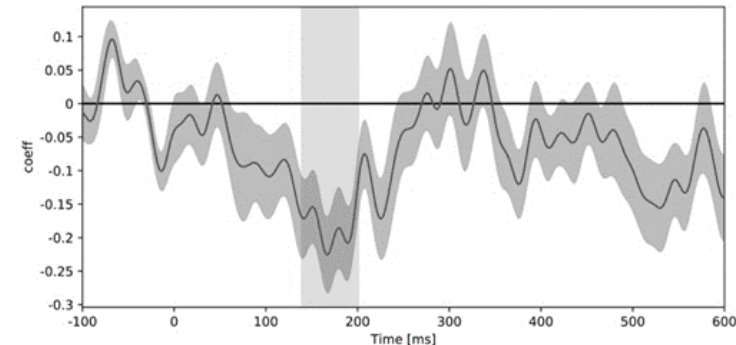

Figure 1. Experimental stimuli and the left FG/ITG activations. (A) Morpheme-letter mismatch in Japanese. (B) Significant M170 in the left FG/ITG. (C) M170 modulated by the morphTP.

\section{Results}

Behavioral data showed that the mean accuracies of the lexical decision task of intransitives, transitives, intransitive-causatives, and transitive-causatives were higher than $90 \%$, indicating that the participants correctly performed this task. A two-way repeated-measures analysis of variance (causativeness*transitivity) for the accuracies showed significant main effects of causativeness and transitivity, as well as an interaction (causativeness: $p<0.0001$, transitivity: $p=0.046$, interaction: $p=0.042$ ). The reaction times also showed a significant effect of causativeness, but neither a main effect of transitivity nor an interaction was significant (causativeness: $p<0.0001$, transitivity: $p=0.39$, interaction: $p=0.34$ ), indicating that the causative conditions were more demanding than non-causative conditions.

For the MEG data, we first examined whether the causative conditions, which were morphologically more complex conditions, modulated activation in the left FG/ITG. We found a significantly larger activation under the causative conditions in the anterior part of the left FG/ITG (corrected $p=0.044$ ) (Figure 1B). On the other hand, the main effect of transitivity was not significant (corrected $\mathrm{p}>0.2$ ).

We further examined whether the morphTP modulated the left FG/ITG activation, by using spatiotemporal cluster regression analyses. We found a significant negative correlation between the morphTP and the activation in the posterior part of the left FG/ITG (corrected $p<0.03$ ) (Figure 1C). In contrast, we did not find any significant correlation between the letterTP and the left FG/ITG activation (corrected $\mathrm{p}>0.2$ ). 


\section{Discussion}

In the present MEG study, we found a significantly larger activation under the causative conditions in the anterior part of the left FG/ITG. Because the TP from the verbal root to the causative suffix was lower than that of the verbal root to a tense suffix, this activation may reflect the difference of the morphTP.

Moreover, we found a significant negative correlation between the morphTP and the left FG/ITG activation, whereas we did not find any significant correlation between the letterTP and the left FG/ITG activation. These results demonstrated that morphologically complex verbs in Japanese are indeed decomposed into morphemes, but not into letters, similar to morphologically complex words in English examined in the previous MEG studies.

\section{References}

Gramfort, A., M. Luessi, E. Larson, D. A. Engemann, D. Strohmeier, C. Brodbeck, R. Goj, M. Jas, T. Brooks, L. Parkkonen, M. Hämäläinen. (2013). MEG and EEG data analysis with MNE-Python. Frontiers in Neuroscience, 7, 267.

Gwilliams, L., G. Lewis, A. Marantz. (2016). Functional characterisation of letter-specific responses in time, space and current polarity using magnetoencephalography. Neuroimage, 132, 320-333.

Lewis, G., O. Solomyak, A. Marantz. (2011). The neural basis of obligatory decomposition of suffixed words. Brain and Language, 118, 118-127.

Oldfield, R. C. (1971). The assessment and analysis of handedness: The Edinburgh inventory. Neuropsychologia, 9, 97-113.

Solomyak, O., and A. Marantz. (2009). Lexical access in early stages of visual word processing: A single-trial correlational MEG study of heteronym recognition. Brain and Language, 108, 191-196.

Solomyak, O., A. Marantz. (2010). Evidence for Early Morphological Decomposition in Visual Word Recognition: A Single-Trial Correlational MEG Study. Journal of Cognitive Neuroscience, 22, 2042-2057. 


\title{
Cortical and subcortical involvement during Object an Action Naming in healthy participants under nTMS
}

\author{
Ann-Katrin Ohlerth ${ }^{1}$, Roelien Bastiaanse ${ }^{1,2}$, Chiara Negwer ${ }^{3}$, Nico Sollmann ${ }^{3,4}$, Severin \\ Schramm $^{3}$, Axel Schröder ${ }^{3} \&$ Sandro M. Krieg ${ }^{3}$ \\ ${ }^{1}$ Center for Language and Cognition Groningen, the Netherlands \\ ${ }^{2}$ National Research University Higher School of Economics, Moscow, Russia \\ ${ }^{3}$ Department of Neurosurgery, Klinikum rechts der Isar, Technische Universität München, \\ Munich, Germany \\ ${ }^{4}$ Department of Diagnostic and Interventional Neuroradiology, Klinikum rechts der Isar, \\ Technische Universität München, Munich, Germany
}

\section{Introduction}

As widely known from aphasia research, language batteries should consist of tasks both targeting noun and verb production in order to reliably test language skills (Rofes, Capasso, \& Miceli, 2015). Whereas this knowledge has largely arrived in the intraoperative language mapping in tumour patients, it is not yet implemented in the adjunct method of preoperative language mapping under navigated Transcranial Magnetic Stimulation (nTMS). This non-invasive brain stimulation method is increasingly used in preoperative assessment: through temporary disruption of small cortical areas, mapping with nTMS results in a cortical language map of positive and negative language areas, that helps planning the operation and in decision making regarding tumour removal (Sollmann et al., 2015).

Recently, advances have been made in nTMS-based fiber tracking, a method where cortical areas found through nTMS function as seeds for revealing language relevant white matter bundles (Sollmann et al., 2016). Through this addition, even subcortical resection can be planned and executed based on the preoperative information and thereby, nTMS language mapping has gained even more clinical relevance.

However, the language batteries used under nTMS for cortical and subcortical mapping are solely based on Object Naming tasks targeting nouns. They lack standardized protocols of profound linguistic knowledge that can reliably test language production. Hence, maps acquired through nTMS only show noun production. Resection based on this may result in damage of other language skills such as production of verbs.

In this study, we present a two-task paradigm of Object and Action Naming for nTMS language mapping tested in 20 healthy participants as a first step towards clinical application. We aim to answer the question whether the verb task Action Naming is a necessary addition to the standard noun task Object Naming by comparing both cortical and subcortical maps.

\section{Methods}

\section{Participants}

20 healthy German participants (mean age=22 years, 11 female) underwent nTMS language mapping of both hemispheres.

\section{Materials}

Two picture naming tasks, that had been standardized in an earlier study, were administered. The tasks Object Naming and Action Naming consist of each 75 items of black-and-white drawings of every objects and actions, accompanied with a lead-in phrase "This is..." / "The man..." triggering target nouns and verbs in sentence context, respectively. 


\section{Procedure}

Before undergoing stimulation mapping, individuals MRIs were made and the participants named all stimuli without stimulation in a baseline naming to remove unsuitable items. During stimulation mapping, the participants named the same items, while being stimulated on the cortex by nTMS. 46 predefined cortical areas, covering both hemispheres, were targeted each 6 times.

\section{Analysis}

Offline video recordings of both naming tests allowed post-hoc analysis to determine whether the stimulation had resulted in one of the following errors (table 1).

Table 1. Error categories and types

\begin{tabular}{ll}
\hline Category & Error types \\
\hline Non-linguistic speech errors & No response; Hesitation on whole sentence \\
Errors at lexico-semantic level & Semantic errors; Anomia; Hesitation on target \\
Errors at morpho-syntactic level & Grammatical errors \\
Errors at phonological/phonetic level & Performance errors \\
\hline
\end{tabular}

The error rates for Object and Action Naming were calculated per hemisphere and per cortical area and compared by Mann-Whitney U tests.

If an error was found, the respective stimulated area was considered language positive. Positive areas were used as seeds for DTI-fiber tracking to reconstruct fiber bundles terminating in said areas.

\section{Results}

Action Naming revealed an overall higher error rate than Object naming $(p=0.02)$. In the area-wise comparison, Action Naming showed a significantly higher error rate in the middle precentral and postcentral gyrus of the right hemisphere. Concerning error types, the overall higher error rate of Action Naming arose from the category of semantic errors $(p=0.008)$. The next step will be nTMS-DTI fiber tracking. These data will be presented as well.

\section{Discussion}

The higher overall error rate of action naming stresses the necessity of adding the task to the paradigm: Action Naming revealed more positive areas, especially around the central sulcus. Moreover, in some individuals hardly any positive areas could be detected with Object Naming, but only with Action Naming, rendering it the more suitable task for preoperative language mapping in these cases. When looking closer into the categories of errors, it was revealed that the significant difference between the two tasks arose mainly from the higher number of lexicosemantic errors. Thus, nTMS affects access to verbs more than access to nouns. This novel information can be supported by the body of literature pointing out the linguistic differences between the two word classes and their corresponding cortical correlates (Vigliocco, Vinson, Druks, Barber, \& Cappa, 2010). Once the DTI-fiber tracking is analyzed, it can be determined how valuable the addition of Action naming to the standard task Object Naming is for subcortical depictions of language relevant fibers.

\section{References}

Rofes, A., Capasso, R., \& Miceli, G. (2015). Verb production tasks in the measurement of communicative abilities in aphasia Verb production tasks in the measurement of com- 
municative abilities in aphasia. Journal of Clinical and Experimental Neuropsychology. https://doi.org/10.1080/13803395.2015.1025709

Sollmann, N., Ille, S., Hauck, T., Maurer, S., Negwer, C., Zimmer, C., ... Krieg, S. M. (2015). The impact of preoperative language mapping by repetitive navigated transcranial magnetic stimulation on the clinical course of brain tumor patients. BMC Cancer. https://doi.org/10.1186/s12885-015-1299-5

Sollmann, N., Negwer, C., Ille, S., Maurer, S., Hauck, T., Kirschke, J. S., ... Krieg, S. M. (2016). Feasibility of nTMS-based DTI fiber tracking of language pathways in neurosurgical patients using a fractional anisotropy threshold. Journal of Neuroscience Methods. https://doi.org/10.1016/j.jneumeth.2016.04.002

Vigliocco, G., Vinson, D. P., Druks, J., Barber, H., \& Cappa, S. F. (2010). Nouns and verbs in the brain: A review of behavioural, electrophysiological, neuropsychological and imaging studies. Neuroscience and Biobehavioral Reviews, 35, 407-426. https://doi.org/10.1016/j.neubiorev.2010.04.007 


\title{
Spontaneous Speech Analysis in Spanish-Speaking Adults: Normative data in healthy adults, elderly adults and patients with brain lesions
}

\author{
Carolina Méndez-Orellana ${ }^{1}$, Caitlin Holme ${ }^{2}$, Karina Sandoval-León ${ }^{3}$, Bárbara Cortés-Rivera ${ }^{1}$, \\ Paula Méndez-Orellana ${ }^{4}$, Silvia Martínez-Ferreiro ${ }^{5}$ \\ ${ }^{1}$ Carrera de Fonoaudiología, Departamento Ciencias de la Salud, Facultad Medicina, \\ Pontificia Universidad Católica de Chile, Santiago, Chile \\ ${ }^{2}$ Bristol Speech and Language Therapy Research Unit, North Bristol NHS Trust, Bristol, \\ United Kingdom \\ ${ }^{3}$ Escuela de Fonoaudiología, Facultad de Ciencias de la Salud, Universidad Católica Silva \\ Henríquez, Santiago, Chile \\ ${ }^{4}$ Escuela de Fonoaudiología, Facultad de Medicina, Universidad Austral de Chile, Puerto \\ Montt, Chile \\ ${ }^{5}$ Nordic Studies and Linguistics, University of Copenhagen, Copenhagen, Denmark
}

\section{Introduction}

Several methods of spontaneous speech analysis (SSA) have been developed along the years (Saffran et al., 1989; Vermeulen et al., 1989; Boxum et al., 2013; Grande et al., 2008). However, the number of tools available varies across languages. A striking case is that of Spanish which, despite counting with more than 500 million speakers over 21 countries (Benedet et al., 1998) and a growing number of second language learners, still lacks a reliable and standardized tool for the analysis of the speech output of adults with and without brain damage. Normative data to check against potential measurements is also missing, thus increasing the challenge of evaluating language deficits in an effective way, especially in mild and residual cases of aphasia.

In this context, this presentation aims at providing an overview of a method of SSA specially conceived for the quantification of Spanish data in adults: the ALEA (Análisis del Lenguaje Espontáneo en Adultos 'Analysis of Spontaneous Speech in Adults'). Departing from already existing methods, the ALEA, developed at the Speech and Language Therapy department of the Pontificia Universidad Católica de Chile, by an international multidisciplinary team, is envisioned as a tool for health professionals working with adults with a wide array of language disorders to the inclusion of (but not limited to) aphasias of diverse etiology, dementia, and mild cognitive impairment.

The ALEA addresses main issues regarding SSA, namely: a) the lack of consistency across raters and methods that can compromise reliability (Edwards, Garman \& Knott, 1993), b) the lack of systematicity in the criteria for the selection of the variables under scrutiny, and c) the laborious process of data analysis, which in some cases include the quantification of subjective variables.

\section{Methods \& Results}

Design

Most of the methodology of the ALEA has been adapted from the ASTA (Boxum et al., 2013), designed for Dutch speakers. Following its guidelines, changes were introduced to accommodate linguistic differences and favor its use in clinical contexts, turning it into an easy to administer, sensitive tool for the identification of language difficulties in adults. The indices considered in ALEA are summarized in table 1. 
Table 1. Description of ALEA Linguistic Variables with SSA method and rationale as justification.

\begin{tabular}{|c|c|c|c|}
\hline Linguistic Variable & Calculation & SSA Method & Rationale \\
\hline 1. MLU & $\begin{array}{l}\text { No. analysis words/total } \\
\text { no. utterances }\end{array}$ & $\begin{array}{l}\text { ASTA (Boxum et } \\
\text { al., 2013) }\end{array}$ & $\begin{array}{l}\text { Useful in distinguishing aphasia from } \\
\text { control speech }\end{array}$ \\
\hline $\begin{array}{l}\text { 2. Approximation } \\
\text { Index }\end{array}$ & $\begin{array}{l}\text { No. repetitions }+ \text { false } \\
\text { starts }+ \text { filler words / no. } \\
\text { words transcribed }\end{array}$ & Satoer et al. (2013) & $\begin{array}{l}\text { Glioma patients deviated from controls } \\
\text { on repetitions }\end{array}$ \\
\hline $\begin{array}{l}\text { 3. No. paraphasias } \\
\text { and / or neologisms }\end{array}$ & $\begin{array}{l}\text { No. paraphasias }+ \text { neolo- } \\
\text { gisms }\end{array}$ & $\begin{array}{l}\text { ASTA (Boxum et } \\
\text { al., 2013) }\end{array}$ & $\begin{array}{l}\text { Useful in distinguishing aphasia from } \\
\text { control speech }\end{array}$ \\
\hline 4. Nouns & No. nouns per utterance & $\begin{array}{l}\text { QPA (1989); Bird } \\
\text { and Franklin (1996) }\end{array}$ & $\begin{array}{l}\text { Useful for distinguishing people with } \\
\text { fluent aphasia from controls }\end{array}$ \\
\hline 5. Verbs & $\begin{array}{l}\text { No. of verbs per utterance } \\
\text { (incorrect verbs are regis- } \\
\text { tered) }\end{array}$ & $\begin{array}{l}\text { ASTA (Boxum et } \\
\text { al., 2013), Mar- } \\
\text { tinez Ferreiro et al. } \\
(2017)\end{array}$ & $\begin{array}{l}\text { Distinguished between people with } \\
\text { aphasia \& controls; also relevant for } \\
\text { Spanish patients. }\end{array}$ \\
\hline $\begin{array}{l}\text { 6. Finiteness of the } \\
\text { utterances }\end{array}$ & $\begin{array}{l}\text { Percentage of finite } \\
\text { clauses }\end{array}$ & $\begin{array}{l}\text { Martinez Ferreiro } \\
\text { et al. }(2017)\end{array}$ & $\begin{array}{l}\text { Useful distinction across aphasia sever- } \\
\text { ity }\end{array}$ \\
\hline $\begin{array}{l}\text { 7. } \% \text { Incomplete } \\
\text { Utterances }\end{array}$ & $\begin{array}{l}\text { No. incomplete / total no. } \\
\text { utterances }\end{array}$ & $\begin{array}{l}\text { Satoer et al. } \\
(2013) ; \text { Grande et } \\
\text { al. }(2008)\end{array}$ & $\begin{array}{l}\text { Glioma patients deviated from controls } \\
\text { on this measure }\end{array}$ \\
\hline $\begin{array}{l}\text { 8. \% Grammatical- } \\
\text { ity }\end{array}$ & $\begin{array}{l}\text { No. grammatical / total } \\
\text { no. utterances }\end{array}$ & $\begin{array}{l}\text { ASTA (Boxum et } \\
\text { al., 2013) }\end{array}$ & $\begin{array}{l}\text { Useful to distinguish both people with } \\
\text { aphasia and glioma patients from con- } \\
\text { trols }\end{array}$ \\
\hline $\begin{array}{l}\text { 9. Subordination } \\
\text { Index }\end{array}$ & $\begin{array}{l}\text { No. utterances with sub- } \\
\text { ordination/total no. ut- } \\
\text { terances }\end{array}$ & $\begin{array}{l}\text { ASTA (Boxum et } \\
\text { al., 2013) }\end{array}$ & $\begin{array}{l}\text { Adapted (index rather than the total } \\
\text { no. of subordinate clauses); disordered } \\
\text { speech usually has less subordination }\end{array}$ \\
\hline
\end{tabular}

NB: MLU = Mean Length of Utterance; SSA = Spontaneous Speech Analysis; ASTA = Analyse voor Spontane Taal bij Afasie; QPA =Quantitative Production Analysis.

Three steps are necessary to complete the ALEA: 1) obtaining the speech sample, 2) transcribing and annotating the sample, and 3) analyzing the sample. Semi-spontaneous speech samples are elicited using a closed questionnaire. Following standard practice, the speech samples should consist of at least 300 words, including neologisms, paraphasias, and stereotypical speech but excluding repetitions, minimal responses, self-corrections and filler words (e.g. interjections). Once split into utterances, the sample is processed in an online Web application. Different tiers are used to automatize the analysis. A summary of data and the comparison with the norms are analyzed automatically. Detailed descriptions of the variables (including examples) and instructions about annotation are explained in full in the ALEA manual.

\section{Normative and patient data}

The first data collection round focused on adults without neurological damage. Norms were obtained from a sample of 118 healthy individuals (age ranges 28 to 90 years ) who participated voluntarily in the data collection process. As well, data of 60 acute aphasic patients, 15 brain tumor patients were collected to test the clinical applicability of ALEA method. Quantitative and qualitative analysis of patient data is being processed.

\section{Discussion}

The ALEA aims to be a structured tool to gather exhaustive information about the speech output of different adult populations: people with aphasia of varied etiology, healthy aging, mild cognitive impairment, and dementia onset. The main strength of the ALEA is its controlled length and easiness of administration which favors its implementation in the clinical practice. The number of indices are kept to a minimum to provide a first screening of the speech output of different groups of adult speakers. The terminology is simplified to grant comprehension by specialists of different disciplines. The automatized analysis also favors the collection of data, 
keeping errors to a minimum, and the classification of informants according to the linguistic profile based on deviations for the normative data. Additionally, the format of the ALEA allows for the inclusion of new indices (and the corresponding annotation keys) constituting an ideal tool for further in-depth linguistic analyses.

In its present shape, the ALEA is not free from shortcomings. First, as in any other method of analysis, annotation, although kept to a minimum, is still time-consuming. From a purely linguistic perspective, one of the unresolved issues is the use of contractions in Spanish. The most notable cases are illustrated in (1): enclitic pronouns [V $+\mathrm{Cl}$.] and clusters of prepositions and determiners [Prep + Det].
(1) No
quiero
irme
Not want-pres.1st.sg
'(I) don't want
go-INF-me[V + Cl.] to go
al campo. to-the[Prep + Det] countryside to the countryside'.

Spaces between words are crucial for the automatized word counts. Hence, in the first version contractions are automatically computed as a single word. Given that neither pronouns nor prepositions or articles are included among the default indices calculated in ALEA, the issue has so far been left for further developments.

\section{References}

Benedet, M., Christiansen, J., \& Goodglass, H. (1998). A cross-linguistic study of grammatical morphology in Spanish- and English-speaking agrammatic patients. Cortex, 34, 309-336.

Boxum, E., Van der Scheer, F. \& Zwaga, M. (2013). Analyse voor Spontane Taal bij Afasie (ASTA). Standaard in samenwerking met de Vereniging voor Klinische Linguïstiek. 4th version. Retrieved 13/01/17 from: http://www.klinischelinguistiek.nl.

Edwards, S., Garman, M. and Knott, R. (1993). Short report: The grammatical characterization of aphasic language. Aphasiology, 7, 217-220.

Grande, M., Hussmann, K., Bay, E., Christoph, S., Piefke, M., Willmes, K. \& Huber, W. (2008). Basic parameters of spontaneous speech as a sensitive method for measuring change during the course of aphasia. International Journal of Language $\& 6$ Communication Disorders, 43, 408-426.

Saffran, E.M., Berndt, R.S. \& Schwartz, M.F. (1989). The quantitative analysis of agrammatic production: Procedure and data. Brain and Language, 37, 440-479.

Vermeulen, J., Bastiaanse, R. \& Wageningen, B. (1989). Spontaneous output in aphasia: A correlational study. Brain and Language, 36, 252-274. 